\title{
Real change or natural fluctuation?
}

Citation for published version (APA):

Hilderink, J. M. (2021). Real change or natural fluctuation? Linking laboratory results to clinical practice. [Doctoral Thesis, Maastricht University]. Maastricht University. https://doi.org/10.26481/dis.20210330jh

Document status and date:

Published: 01/01/2021

DOI:

10.26481/dis.20210330jh

Document Version:

Publisher's PDF, also known as Version of record

\section{Please check the document version of this publication:}

- A submitted manuscript is the version of the article upon submission and before peer-review. There can be important differences between the submitted version and the official published version of record.

People interested in the research are advised to contact the author for the final version of the publication, or visit the DOI to the publisher's website.

- The final author version and the galley proof are versions of the publication after peer review.

- The final published version features the final layout of the paper including the volume, issue and page numbers.

Link to publication

\footnotetext{
General rights rights.

- You may freely distribute the URL identifying the publication in the public portal. please follow below link for the End User Agreement:

www.umlib.nl/taverne-license

Take down policy

If you believe that this document breaches copyright please contact us at:

repository@maastrichtuniversity.nl

providing details and we will investigate your claim.
}

Copyright and moral rights for the publications made accessible in the public portal are retained by the authors and/or other copyright owners and it is a condition of accessing publications that users recognise and abide by the legal requirements associated with these

- Users may download and print one copy of any publication from the public portal for the purpose of private study or research.

- You may not further distribute the material or use it for any profit-making activity or commercial gain

If the publication is distributed under the terms of Article $25 \mathrm{fa}$ of the Dutch Copyright Act, indicated by the "Taverne" license above, 


\section{Real change or \\ natural fluctuation?}

Linking laboratory results to clinical practice 


\section{Real change or natural fluctuation?}

Linking laboratory results to clinical practice Judith M. Hilderink

Cover design by: Sanne Kassenberg (Persoonlijk Proefschrift)

Layout by: Sanne Kassenberg (Persoonlijk Proefschrift)

Printed by: Gildeprint

ISBN: 978-94-6419-159-2

Copyright $\odot$ Judith M. Hilderink, Rotterdam 2021

No part of this book may be reproduced or transmitted in any form or by any means, without prior permission in writing by the author or, when appropriate, by the publishers of the publication 


\section{Real change or natural fluctuation?}

Linking laboratory results to clinical practice

\section{PROEFSCHRIFT}

ter verkrijging van de graad van doctor aan de Universiteit Maastricht op gezag van de Rector Magnificus, Prof. dr. Rianne M. Letschert, volgens het besluit van het College van Decanen, in het openbaar te verdedigen

op dinsdag 30 maart 2021 om 13:00 uur

door

Judith Maria Hilderink

Geboren 4 oktober 1990

te Hengelo (Gld.) 


\section{Promotores}

Prof. dr. O. Bekers

Prof. dr. R.P. Koopmans

\section{Copromotor}

Dr. S.J.R. Meex

\section{Beoordelingscommissie}

Prof. dr. W.N.K.A. van Mook (voorzitter)

Prof. dr. J.J. Rethans

Prof. dr. F. Sweep (Radboud Universiteit, Nijmegen)

Prof. dr. A.A. Kroon

Prof. dr. E.J.G. Sijbrands (Erasmus MC, Rotterdam)

The research described in this thesis was supported by an Academic Incentive Grant from Maastricht University. 


\section{Table of contents}

$\begin{array}{lll}\text { Chapter } 1 & \text { General introduction } & 7\end{array}$

$\begin{array}{lll}\text { Chapter } 2 & \text { Real change or natural fluctuation? } & 17\end{array}$

Chapter 3 Within-day biological variation and hour-to-hour reference change values of hematological parameters

Chapter 4 Within-day biological variation of 26 biochemical 65 parameters in subjects with- or without chronic kidney disease

Chapter 5 Twenty-four hour biological variation of creatinine, 95 cystatin C and estimated Glomerular Filtration Rate in subjects with or without Chronic Kidney Disease

Chapter 6 Twenty-four hour biological variation profiles of cardiac 119 troponin I in subjects with or without chronic kidney disease

Chapter 7 Biological variation of cardiac markers in patients with 133 aortic valve stenosis

Chapter 8 Labtracker, a medical smartphone app for the 149 interpretation of consecutive laboratory results: an external validation study

Chapter 9 General discussion

$\begin{array}{lll}\text { Addendum Summary } & 182\end{array}$

Samenvatting $\quad 184$

Impact paragraph $\quad 186$

List of abbreviations 188

List of publications 190

Curriculum Vitae 192

Dankwoord 193 


\section{Chapter 1}




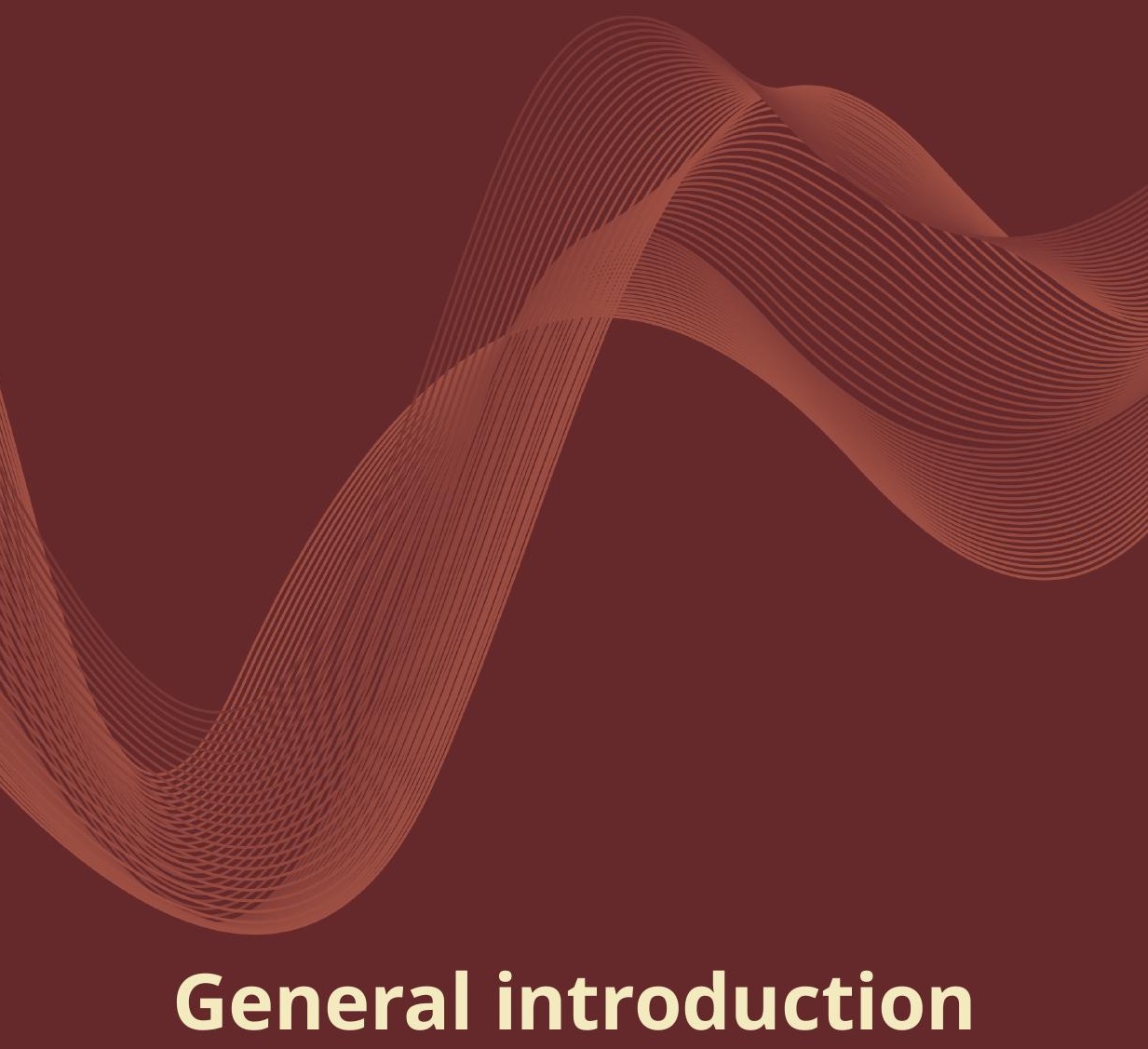




\section{General introduction}

The clinical challenge

Laboratory results serve at least two major goals: 1) Diagnosis or exclusion of disease and 2) monitoring changes in the clinical condition of a patient (1-4). Diagnosing or exclusion of disease benefits from the availability of populationbased reference intervals (5-8). For monitoring of patients however, populationbased reference intervals have no or limited utility, because the variability between serial measurements that is considered normal in an individual, is generally much smaller than the population-based reference interval (8). Take serum creatinine as an example: a change in creatinine from 65 to $105 \mu \mathrm{mol} / \mathrm{L}$ does not exceed the population-based reference interval on either side, but nevertheless represents a highly significant change within an individual. This phenomenon applies to most parameters that can be measured in laboratory medicine, and illustrates why population-based reference values have limited value when monitoring patients over time (8-10).

\section{Population-based reference values}

A population-based reference interval typically includes $95 \%$ of test results obtained from a healthy population $(11,12)$. As such, it represents the spectrum of values for a given parameter that is considered "normal", and population-based reference values (or "normal values") are probably the most commonly applied interpretative aid in diagnosis and case finding in clinical practice $(8,13)$. By definition, $5 \%$ of healthy subjects will have results outside the population-based reference interval. This percentage rapidly increases with each additional laboratory test being conducted. Awareness of this limitation of reference intervals is useful, as it may trigger unnecessary further investigation and over-testing (14-16).

Since population-based reference values are based on the normal variation between subjects, they are generally not suited to interpret changes in serial measurements within an individual for the purpose of patient monitoring over time $(8,17)$. As demonstrated in the creatinine example above, normal biomarker variation within an individual is usually much lower than normal variation between individuals. Each individual has its own "homeostatic set point" for a biomarker $(6,8)$. Day-to-day or hour-to-hour biomarker concentrations fluctuate randomly around this homeostatic set point within individuals (18-21). This is called withinsubject biological variation. Some biomarkers exhibit relatively large variability around their homeostatic set point e.g. triglycerides, whereas for other biomarkers 
the range permitted by normal homeostasis of the body is very small (e.g. sodium). Homeostatic set points differ between individuals, and may span the full population-based reference range (8). An individual's homeostatic set point can increase or decrease, e.g due to aging or a chronic disease, but the variability around the homeostatic set point is believed to be relatively stable, and similar across individuals $(8,22-24)$.

When conducting serial measurements in a person who has a homeostatic set point close to the lower bound of the reference range, a slightly lower second measurement may turn a result from 'normal' to 'abnormal' (according to reference range values), although the result may have hardly changed from the previous value (25-27). On the other hand, a significant (and clinically relevant) rise in this individual may give a result that does not yet cross the upper bound and remains within the reference interval. Such change however may well require further investigation. Thus, when using population-based reference values for monitoring purposes, normal fluctuation in parameters may erroneously be flagged as abnormal, while significant changes may not be flagged and remain unnoticed.

\section{Monitoring test results over time.}

The much less known counterpart of population-based reference values, developed for the interpretation of serial measurements, is the "reference change value" or "critical difference" $(25,28)$. For a change between serial measurements to become significant, the difference between the two measurements must exceed the total inherent variation (29). The reference change value is the minimal difference between serial measurements that can be considered a statistically significant change. Quantitative test results vary over time, even without a change in the patient's clinical status, due to three sources of inherent variation: 1) pre-analytical variability (e.g. delays in centrifugation, temperature changes prior to analysis, delay in tube transport to the lab), 2) analytical variability (random error) in the measurement itself, and 3) biological variability $(6-8,30)$.

Pre-analytical variation, such a sample collection and sample handling, can be well standardized by training and adherence to standard operating procedures, and is considered negligible compared to the other two sources of variation (31). Analytical variation is intrinsic to the measurement technique. Each laboratory measurement is associated with random error of which the magnitude depends 
on the methodology and analyzer used (32). Analytical variation is considered acceptable when it is $\leq 50 \%$ of the biological variation, and in laboratory medicine this ratio is an important quality criterion for the desired precision of an assay (10, 33). Finally, biological variation refers to random fluctuation around an individuals' homeostatic set point due to fasting status, hydration status, recent exercise, cyclical rhythms etcetera. All three parameters together contribute to day-to-day variability in test results and influence the probability that a change between serial laboratory measurements is "real" or "random fluctuation" $(10,17,34)$.

The reference change value (RCV) can be easily calculated: within-subject biological variation $\left(\mathrm{CV}_{\mathrm{I}}\right)$ is relatively constant, and has been studied and published for a number of laboratory parameters. The analytical variation $\left(\mathrm{CV}_{\mathrm{A}}\right)$ for each parameter is available on request from clinical laboratories.

$$
R C V=\sqrt{2} * Z * \sqrt{C V_{A}^{2}+C V_{I}^{2}}
$$

In this formula Z represents the number of standard deviations and corresponds to the desired probability (8). Any probability can be chosen here, but often used Z-scores are 1.96 en 2.58. With these values one calculates the percentage rise or fall required to reach significance, while accepting the chance of a false positive change of $5 \%(p \leq 0.05)$ and $1 \%(p \leq 0.01)$, respectively (35).

\section{Using Reference Change values in medical decision making}

Although reference change values are a well-established concept in laboratory medicine, its use is hardly implemented in clinical practice (36). One reason may be that many medical decisions are not taken at fixed probability levels (e.g. $p<0.05$ or $\mathrm{p}$ 0.01). Often, changes between serial measurements do not reach significance from a statistical perspective, but are still sufficiently large to influence a clinical decision. Hence, the classical RCV-formula that only indicates high (and fixed) probability level is less convenient in daily practice. A more informative outcome would be to derive the probability of change between any two serial measurements. This can be easily achieved through a simple rearrangement of the RCV formula that makes the Z-score (and hence the probability) the unknown:

$$
\text { Z-score }=\frac{\text { percentage change between consecutive measurements }}{\sqrt{2 *\left(C V_{A}^{2}+C V_{I}^{2}\right)}}
$$


This rearrangement allows the calculation of the probability as a percentage between $0 \%$ and $100 \%$ between any two serial measurements (8).

\section{Outline of this thesis}

Population-based reference intervals, based on between-subject biological variations are generally unsuitable for monitoring of serial lab results. Availability of data on within-subject biological variation, which is a pre-requisite for the calculation of a reference change value is incomplete, limiting evidence-based interpretation of serial laboratory measurements in clinical practice. In addition, the classical concept of the reference change values is primarily a laboratory tool, which is not user-friendly in clinical practice. Bridging the gap between the theoretical RCV concept in laboratory medicine and its translation to a user-friendly medical application would help clinicians discriminate between real changes and random fluctuation when interpreting serial laboratory results. This thesis aims to 1) extend the availability of within-subject biological variation data, and 2) translate raw biological variation data to an educational/medical software device, based on the rearranged RCV formula as described above.

To translate the scientific concept of biological variation to clinical practice, a medical smartphone app was developed: Labtracker. In chapter $\mathbf{2}$, the background and underlying calculation algorithm of the app are described.

Chapter 3 reports the within-day and hour-to-hour biological variation of 21 hematological parameters. In subsequent chapters, the biological variation data and RCV's were compared in healthy subjects versus various chronic diseases, for a broad set of laboratory parameters, including general clinical chemistry parameters (chapter 4), parameters of renal function (chapter 5), troponin I (chapter 6), and various novel cardiac markers (hsTnI, hsTnT and ST2) in subjects with aortic valve stenosis (chapter 7)

Chapter 8 reports data of a validation study with Labtracker: concordance rates of Labtracker-based calculations were compared with intuitive assessments by medical students, clinical fellows and experienced clinicians. The final chapter, chapter 9, provides a general discussion of the work presented in this thesis, as well as directions for future research. 


\section{References}

1. Molinaro RJ, Winkler AM, Kraft CS, Fantz CR, Stowell SR, Ritchie JC, et al. Teaching laboratory medicine to medical students: Implementation and evaluation. Arch Pathol Lab Med 2012;136:1423-9.

2. Fan SL, Miller NS, Lee J, Remick DG. Diagnosing sepsis - the role of laboratory medicine. Clin Chim Acta 2016;460:203-10.

3. Oosterhuis WP, Zerah S. Laboratory medicine in the european union. Clin Chem Lab Med 2015;53:5-14.

4. Cornes MP, Church S, van Dongen-Lases E, Grankvist K, Guimaraes JT, Ibarz M, et al. The role of european federation of clinical chemistry and laboratory medicine working group for preanalytical phase in standardization and harmonization of the preanalytical phase in europe. Ann Clin Biochem 2016;53:539-47.

5. Petersen PH, Fraser CG, Sandberg S, Goldschmidt H. The index of individuality is often a misinterpreted quantity characteristic. Clin Chem Lab Med 1999;37:655-61.

6. Fraser CG. Quality goals: Generation and application. Scand J Clin Lab Invest Suppl 1990;202:65-6.

7. Fraser CG. Generation and application of analytical goals in laboratory medicine. Ann Ist Super Sanita 1991;27:369-75.

8. Fraser CG. Biological variation: From principles to practice. Amer Assoc for Clinical Chemistry 2001.

9. Ricos C, Alvarez V, Perich C, Fernandez-Calle P, Minchinela J, Cava F, et al. Rationale for using data on biological variation. Clin Chem Lab Med 2015;53:863-70.

10. Fraser CG, Harris EK. Generation and application of data on biological variation in clinical chemistry. Crit Rev Clin Lab Sci 1989;27:409-37.

11. Bugdayci G, Oguzman H, Arattan HY, Sasmaz G. The use of reference change values in clinical laboratories. Clin Lab 2015;61:251-7.

12. Ahmed S, Curtis S, Hill C, Hine T. Population-based estimated reference creatinine values: A novel method of a robust electronic acute kidney injury alert system. Nephron Clin Pract 2014;128:166-70.

13. Ceriotti F, Hinzmann R, Panteghini M. Reference intervals: The way forward. Ann Clin Biochem 2009;46:8-17.

14. Erard Y, Del Giorno R, Zasa A, De Gottardi S, Della Bruna R, Keller F, et al. A multi-level strategy for a long lasting reduction in unnecessary laboratory testing: A multicenter before and after study in a teaching hospital network. Int J Clin Pract 2018:e13286.

15. Ko SQ, Quah P, Lahiri M. The cost of repetitive laboratory testing for chronic disease. Intern Med J 2019;49:1168-70.

16. van de Wijngaart DL SJ, van den Broek L, van Dijk N, Janssens PMW. A survey of doctors reveals that few laboratory tests are of primary importance at the emergency department. Diagnosis (Berl) 2014;Sep 1;1:239-44.

17. Braga F, Panteghini M. Generation of data on within-subject biological variation in laboratory medicine: An update. Crit Rev Clin Lab Sci 2016;53:313-25.

18. Coskun A, Carobene A, Kilercik M, Serteser M, Sandberg S, Aarsand AK, et al. Within-subject and between-subject biological variation estimates of 21 hematological parameters in 30 healthy subjects. Clin Chem Lab Med 2018;56:1309-18.

19. Carobene A, Aarsand AK, Guerra E, Bartlett WA, Coskun A, Diaz-Garzon J, et al. European biological variation study (eubivas): Within- and between-subject biological variation data for 15 frequently measured proteins. Clin Chem 2019;65:1031-41. 
20. Jones GRD. Estimates of within-subject biological variation derived from pathology databases: An approach to allow assessment of the effects of age, sex, time between sample collections, and analyte concentration on reference change values. Clin Chem 2019;65:579-88.

21. Coskun A, Braga F, Carobene A, Tejedor Ganduxe X, Aarsand AK, Fernandez-Calle P, et al. Systematic review and meta-analysis of within-subject and between-subject biological variation estimates of 20 haematological parameters. Clin Chem Lab Med 2019;58:25-32.

22. Roraas T, Stove B, Petersen PH, Sandberg S. Biological variation: Evaluation of methods for constructing confidence intervals for estimates of within-person biological variation for different distributions of the within-person effect. Clin Chim Acta 2017;468:166-73.

23. Roraas T, Stove B, Petersen PH, Sandberg S. Biological variation: The effect of different distributions on estimated within-person variation and reference change values. Clin Chem 2016;62:725-36.

24. Roraas T, Petersen PH, Sandberg S. Confidence intervals and power calculations for withinperson biological variation: Effect of analytical imprecision, number of replicates, number of samples, and number of individuals. Clin Chem 2012;58:1306-13.

25. Fraser CG. Reference change values. Clin Chem Lab Med 2011;50:807-12.

26. Fuentes-Arderiu X, Padro-Miquel A, Rigo-Bonnin R. Disadvantages of using biological variation data for reference change values. Clin Chem Lab Med 2011;50:961; author reply 3-4.

27. Cheuvront SN, Fraser CG, Kenefick RW, Ely BR, Sawka MN. Reference change values for monitoring dehydration. Clin Chem Lab Med 2011;49:1033-7.

28. Ko DH, Park HI, Hyun J, Kim HS, Park MJ, Shin DH. Utility of reference change values for delta check limits. Am J Clin Pathol 2017;148:323-9.

29. Fraser CG. Inherent biological variation and reference values. Clin Chem Lab Med 2004;42:758-64.

30. Garcia-Panyella M, Padro-Miquel A, Dot-Bach D, Fuentes-Arderiu X. Pre-analytical variation of some haematological quantities. Clin Chem Lab Med 2008;46:1168-70.

31. Fuentes-Arderiu X, Acebes-Frieyro G, Gavaso-Navarro L, Castineiras-Lacambra MJ. Premetrological (pre-analytical) variation of some biochemical quantities. Clin Chem Lab Med 1999;37:987-9.

32. Ricos C, Alvarez V, Minchinela J, Fernandez-Calle P, Perich C, Boned B, et al. Biologic variation approach to daily laboratory. Clin Lab Med 2017;37:47-56.

33. Bailey D, Bevilacqua V, Colantonio DA, Pasic MD, Perumal N, Chan MK, Adeli K. Pediatric within-day biological variation and quality specifications for 38 biochemical markers in the caliper cohort. Clin Chem 2014;60:518-29.

34. Harding PJ, Fraser CG. Biological variation of blood acid-base status: Consequences for analytical goal-setting and interpretation of results. Clin Chem 1987;33:1416-8.

35. Siest G, Henny J, Grasbeck R, Wilding P, Petitclerc C, Queralto JM, Hyltoft Petersen P. The theory of reference values: An unfinished symphony. Clin Chem Lab Med 2013;51:47-64.

36. Lund F, Petersen PH, Fraser CG, Soletormos G. Different percentages of false-positive results obtained using five methods for the calculation of reference change values based on simulated normal and In-normal distributions of data. Ann Clin Biochem 2016;53:692-8. 


\section{Chapter 2}




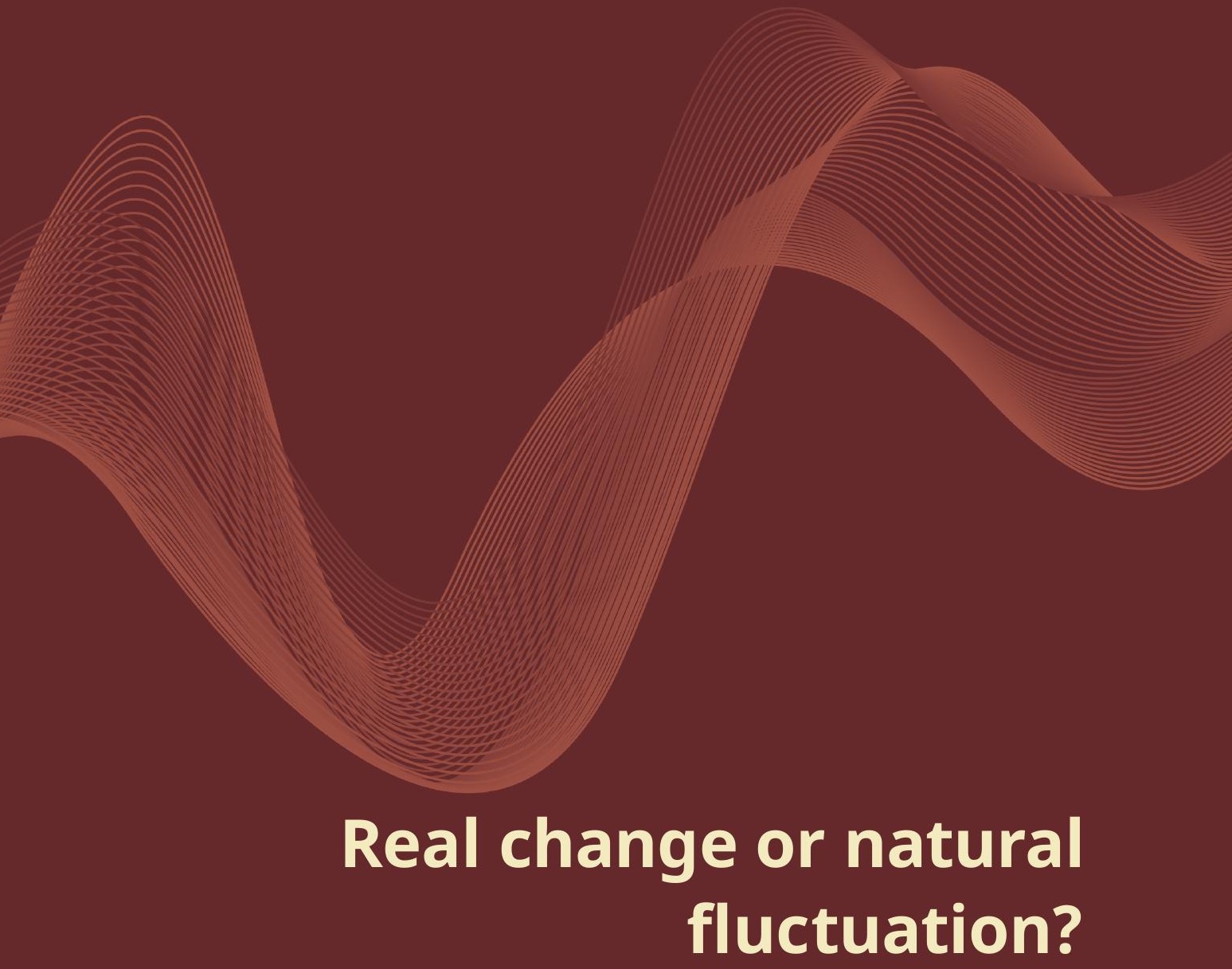

Hilderink JM, Koopmans RP, Rennenberg RJ, van Dieijen-Visser MP, Meex SJ.

Adapted from below publications: Hilderink JM, Koopmans RP, Rennenberg RJMW, van Dieijen-Visser MP en Meex SJR. Real change or natural fluctuation? Ned Tijdschr Geneeskd. 2016;160(0):D132

and

Hilderink JM. Calculating the probability of change between two consecutive laboratory test results. [Reply to: Explaining laboratory test results to patients: what the clinician needs to know by Maurice John O'Kane and Berenice Lopez]. BMJ. 2015;351:h5552. 


\begin{abstract}
When following patients over time, it may be difficult to distinguish 'real changes' in consecutive blood parameters from so-called 'natural fluctuations'. Consider a patient whose cholesterol level decreased from $6.6 \mathrm{mmol} / \mathrm{L}$ to $6.1 \mathrm{mmol} / \mathrm{L}$ in six months after receiving lifestyle advice. How likely is it that this is a 'real change' and that the advice is working?
\end{abstract}

Physicians mostly rely on their intuition and clinical experience when interpreting changes in consecutive laboratory results. For inexperienced physicians, the lack of an easy reference for the interpretation of consecutive laboratory results can be challenging. However, it is possible to objectify the interpretation of these results. We have developed a medical smartphone app that does so. It produces the probability that a change over time in a specific laboratory parameter is 'real', given the time between measurements. It provides more objective input for physicians treating patients such as the one above.

It is a well-known phenomenon: a lab test result that is just slightly too high or too low and when the test is repeated a few months later, the value has dropped or risen. How much attention should you pay to such a change? Is it a 'real' change or merely a physiological occurrence? Two cases are presented below. 


\section{Case 1}

Patient A, a 55-year-old man, visits his general practitioner (GP) for a health check at his own initiative. His blood cholesterol concentration is $6.6 \mathrm{mmol} / \mathrm{L}$ (reference value: 5.0-6.4). The GP determines the patient's cardiovascular risk profile and decides not to prescribe any cholesterol medication for the time being. The GP does provide lifestyle advice. The patient returns after six months. During this second consultation, the cholesterol concentration is determined once again. It is now $6.1 \mathrm{mmol} / \mathrm{L}$.

What are the chances that this patient's cholesterol level has 'really' dropped and, therefore, is not a reflection of a change in the cholesterol concentration in the blood due to physiological fluctuations?
1 A Unlikely (<50\% chance)
1B Dubious (50-80\% chance)
1C Likely (< 80-95\% chance)
1D Very likely (> 95\% chance)

\section{Case 2}

Patient B, a 35-year-old woman, visits the general practice because of fatigue since six months. The GP checks her thyroid function and arrives at the following results (reference values between brackets): TSH: $6.4 \mathrm{mU} / \mathrm{I}$ (0.4-4.3); free thyroxine (FT4): $14.2 \mathrm{pmol} / \mathrm{l}$ (9-24). The GP diagnoses 'subclinical hypothyroidism', but explains to the patient that fatigue is usually not a symptom. After 3 months, the GP checks the thyroid function again. The TSH concentration is now $7.5 \mathrm{mU} / \mathrm{l}$.

To what extent is it likely that the TSH concentration in this patient has 'really' changed and so is not a reflection of a change in the TSH level due to physiological fluctuations?

2A Unlikely ( $<50 \%$ probability)

2B Dubious (50-80\% probability)

2C Likely ( $<80-95 \%$ probability)

2D Very likely (> 95\% probability)

Answer to case 1: answer $1 \mathrm{~B}$ is correct Answer to case 2: answer 2A is correct 
Awareness of day-to-day variability within an individual is particularly important when monitoring disease progression or response to treatment. Importantly, reference values have limited value in the setting of monitoring as they are based on variation between individuals, whereas the interpretation of consecutive measurements requires information on the day-to-day variation within an individual.

Changes in a patient's consecutive laboratory results may be due to clinical improvement or deterioration, but they can also be caused by natural fluctuations (biological variation) or measurement uncertainty (analytical variation). Although experienced physicians rely on their intuition and clinical experience to distinguish 'real' changes from fluctuations of a coincidental nature, this intuitive approach can, in fact, be objectified by calculating the so-called 'critical difference'.

For a change between consecutive measurements to become significant, the difference must be larger than the change that can reasonably be expected due to normal biological and analytical variation. This threshold value is termed the Reference Change Value (RCV). The RCV can be calculated for each laboratory test and depends on the biological within-person variability $\left(\mathrm{CV}_{\mathrm{I}}\right)$ and the analytical variability $\left(\mathrm{CV}_{\mathrm{A}}\right)(1,2)$.

$$
R C V=\sqrt{2} * Z * \sqrt{C V_{A}^{2}+C V_{I}^{2}}
$$

In this formula, the Z-score represents the number of standard deviations and correspond to the desired probability. Commonly used Z-scores are 1.96 and 2.56. These Z-scores calculate the percentage increase or decrease that is required to become statistically significant, with a false positive rate of $5 \%,(p<0.05)$ and $1 \%$ $(p<0.01)$ respectively.

For easy calculation of the RCV we developed Labtracker (3), a free, CE certified, medical smartphone app for iOS and Android. The RCV principle is used to calculate the probability of a true change between serial laboratory results. Over 100 laboratory parameters are currently available in Labtracker. Decision support by Labtracker, using the RCV principle, may be a useful addition to clinical intuition. 


\section{The critical difference}

There is no single critical difference; it varies according to the lab test results. It primarily depends on 2 types of variation: (a) the physiological fluctuation of the relevant parameter in individuals (intra-individual biological variation, $\mathrm{CV}_{\mathrm{I}}$ ); and (b) the measurement uncertainty of the relevant test result (analytical variation, $\mathrm{CV}_{\mathrm{A}}$ ). The critical difference, or reference change value (RCV) is easy to calculate since the intra-individual biological variation is quite constant and has been examined and reported for a great many laboratory test results (3). The laboratories know the analytical variation for each measurement.

$$
R C V=\sqrt{2} * Z * \sqrt{C V_{A}^{2}+C V_{I}^{2}}
$$

Frequently used Z-scores are 1.96 and 2.58. These Z-scores are used to calculate the required percentage increase or decrease for which the chance of a false positive change of $5 \%(p<0.05)$ and $1 \%(p<0.01)$ respectively is acceptable.

If we take a Z-score of 1.96, we can work out that the critical difference for patient A's cholesterol concentration is $21 \%$ and for patient B's TSH concentration $60 \%$. The percentage changes in cholesterol (7.6\%) and TSH (17.2\%) are smaller than the calculated critical differences and, therefore, statistically insignificant.

\section{Statistical significance versus clinical relevance}

In calculating a critical difference, it is important to note that not all medical decisions are based on statistical probabilities of $\geq 95 \%$ or $\geq 99 \%$. In other words: some changes are strictly speaking not statistically significant, but great enough to justify clinical treatment or therapy.

An example here is a patient whose hemoglobin concentration is measured at 6.1 $\mathrm{mmol} / \mathrm{L}$. After a week, it has fallen to $5.8 \mathrm{mmol} / \mathrm{L}$. Although the probability of a 'real' decrease in this patient is less than 95\% - namely approximately $70 \%$ - their physician might be inclined towards further diagnostic investigation or treatment.

In practice, therefore, it is more useful to rephrase the formula for the critical difference so that the Z-score - and thus the probability - will be the unknown (see 'calculating the probability' for this second formula). This second formula can then 
be used to calculate the probability of a change being 'real'. If we do so for patients $A$ and $B$, the probability of a real change works out to be $53 \%$ and $43 \%$ respectively.

\section{Labtracker smartphone app}

The calculation above is rather time-consuming in daily practice. That is why we have incorporated the calculation principle for the critical difference into the Labtracker smartphone app (4-6). This app allows a physician to determine the probability of a change in test results in a very simple way. The outcome is also far more objective compared to the intuitive, mostly experience-based interpretation of lab tests.

The RCV principle is used to calculate the probability of a true change between consecutive laboratory results. Over 100 laboratory parameters are currently available in Labtracker. Decision support by Labtracker, using the RCV principle, may be a useful addition to clinical intuition.

\section{Calculating the probability}

Changes in a patient's consecutive lab results may be due to clinical improvement or deterioration, but can also be caused by natural fluctuation (biological variation) or measurement uncertainty (analytical variation). A change in consecutive results, therefore, may only be viewed as highly likely 'real' when the difference between these results is greater than the critical difference. This is the minimum percentage change between two lab results that can be said to be statistically significant.

But not all decisions are taken when the statistical probability is $95 \%$ or higher. That is why, in practice, it is more useful to rephrase the formula for the critical difference so that the Z-score - and, therefore, the probability - will be the unknown. This new formula can subsequently be used to calculate the probability of a 'real' change:

$$
\text { Z-score }=\frac{\text { percentage change between serial measurements }}{\sqrt{2 * \sqrt{C V_{A}^{2}+C V_{I}^{2}}}}
$$

Case 1; when applied to the case of patient A, it works out that the critical difference of the cholesterol concentration is $21 \%$ (Z-score: 1.96; $\mathrm{CV}_{\mathrm{I}}$ for cholesterol: $6.7 \%$; supposed $\left.\mathrm{CV}_{\mathrm{A}}: 3.4 \%\right)$. A 6.6 change to $6.1 \mathrm{mmol} / \mathrm{L}(7.6 \%)$ is smaller than the calculated critical difference and is, therefore, strictly speaking statistically insignificant. 
The probability for this case is calculated as follows: the percentage change between the first and the second measurements is $7.6 \%, \mathrm{CV}_{\mathrm{I}}$ is $6.7 \%$, and $\mathrm{CV}_{\mathrm{A}}$ is $3.4 \%$. The calculated Z-score is 0.72 . This corresponds to a probability of $53 \%$.

Case 2; the critical difference for the TSH concentration is 60\% (Z-score: 1.96; TSH $\mathrm{CV}_{\mathrm{I}}: 19.3 \%$; supposed $\left.\mathrm{CV}_{\mathrm{A}}: 9.7 \%\right)$. A 6.4 change to $7.5 \mathrm{mU} / \mathrm{L}(17.2 \%)$ in the case of patient $B$ is smaller than the calculated critical difference and so, once again, statistically insignificant.

The probability for this case is calculated as follows: the percentage change between the first and the second measurements is $17.2 \%, \mathrm{CV}_{\mathrm{I}}$ is $19.3 \%$, and $\mathrm{CV}_{\mathrm{A}}$ is $9.7 \%$. The calculated Z-score here is 0.56 . This corresponds to a probability of $43 \%$.

In the calculation of the probability in the Labtracker smartphone app, the percentage figure is expressed in a 'probability term', which varies from 'unlikely'(< $50 \%$ ) to 'highly likely' (>95\%) (6). The application of semantics to probability using different clarifying terms has been described earlier (5). The semantics used for the probability percentages in Labtracker were determined by the authors.

\section{Reference values}

Classic reference or 'normal' values are useful in setting diagnoses and in a situation where no historical lab results are available for a certain patient. But when a patient is followed over the course of time, the usability of reference values is limited, for reference values are based on the biological variation as existing between individuals. For monitoring a patient over time, it is, in fact, the biological variation within an individual that is important. This within-person variation is usually smaller than the variation between people. The fictitious experiment in Figure 2.1 clarifies this phenomenon.

The distribution of the values that are 'normal' for an individual is usually just a small part of the reference value framework for the entire population. It may well be, therefore, that a patient's consecutive lab results show a statistically significant difference - and that this change is also clinical relevant - but that all the values are still within the reference value framework for the population (see Figure 2.1). Quite the opposite can also occur: a patient's consecutive results first fall within and later outside the reference value framework (or the other way around), without this change having any statistical significance or clinical relevance. 
The average value of an individual's certain lab parameter is referred to as the individual equilibrium concentration. This person's values fluctuate around this concentration. Figure 2.1 shows that the equilibrium concentration differs for each individual. The equilibrium concentration may sometimes shift, due to illness or old age, for instance, but the distribution around the individual equilibrium concentration is quite constant (5).

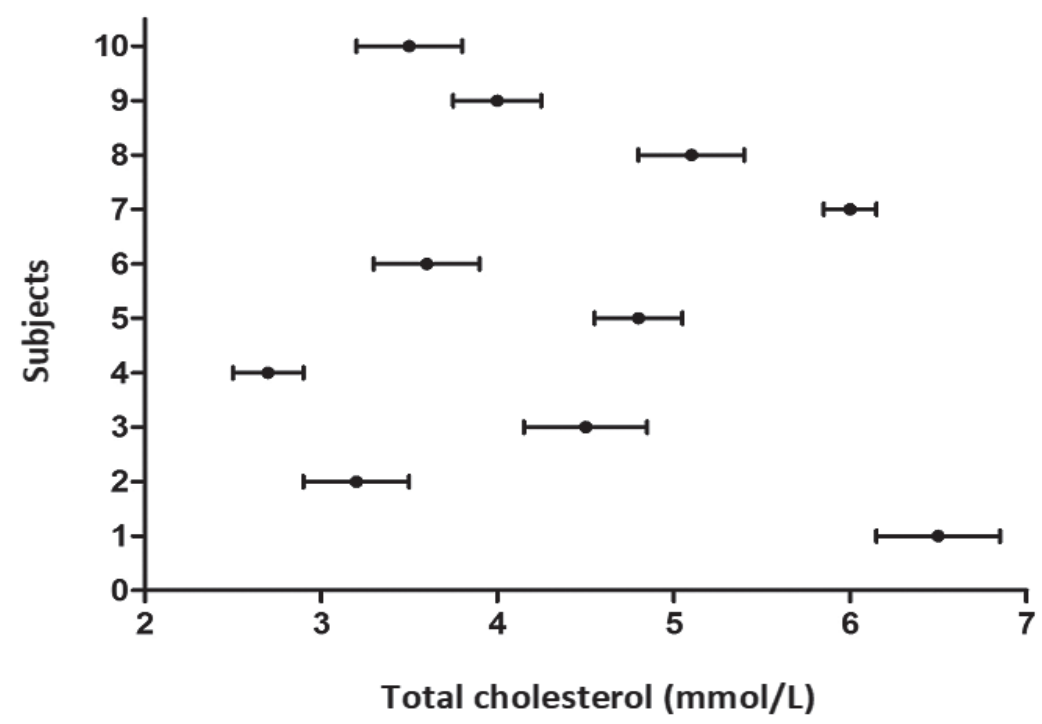

Figure 2.1. In a fictitious experiment, the serum cholesterol concentration is determined every month for a year in 10 apparently healthy persons. The average cholesterol concentration of each individual (equilibrium concentration) and the absolute distribution of values around this concentration are shown. This range reflects the intra-individual biological variation of the cholesterol concentration, that is, the inherent natural fluctuation around an individual's equilibrium concentration. This fluctuation is caused by, for example, diet, physical activity, the day-night rhythm or seasonal influences. The magnitude of the fluctuation strongly determines how much 2 laboratory results must differ from each other to be statistically significant: the critical difference. The figure also shows that the spread between people is much larger than the spread between people; this applies to most laboratory parameters. The differences between the individual equilibrium concentrations are called the inter-individual biological variation and form the basis of population reference values

\section{Time-dependent relationship}

For a number of lab tests, the intra-individual biological variation $\left(\mathrm{CV}_{\mathrm{I}}\right)$ changes over time. The $\mathrm{CV}_{\mathrm{I}}$ sometimes increases as the time interval between the measurements increases. 
Here is an example. When someone's cholesterol level is determined twice a day, virtually the same value will be found in both occasions. But as the time interval between the measurements for the same person increase, the measured values will be wider apart without there necessarily having been any clinical changes. In other words: there is a time relationship in the intra-individual biological variation for a number of results. For the laboratory results for which sufficient information about their biological variation has been published, we modeled these time relationships and incorporated them into the calculation algorithm of the smartphone app. For the purpose of illustration, the increase in the biological variation of the total cholesterol value over time is shown in Figure 2.2.

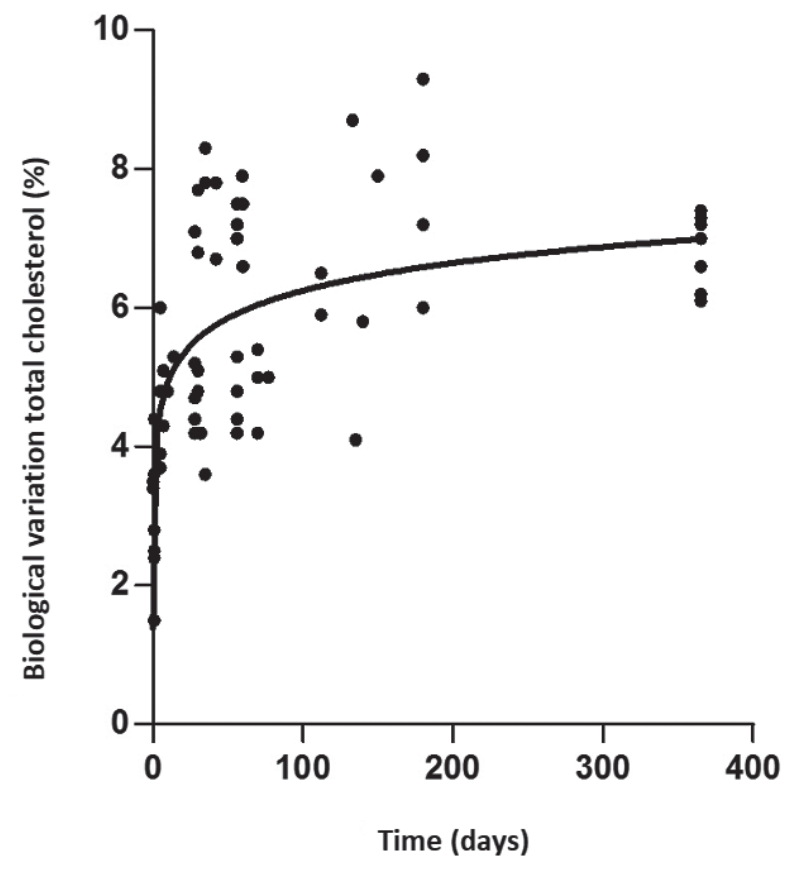

Figure 2.2. There is a clear time relationship between the biological variation and the time between measurements. The biological variation of the total cholesterol value varies from 1.5-9.3\%, depending on the time period between the 2 measurements. Each point in the figure reflects a study on biological variation of this value. The time relationship is modeled as follows and programmed in the Labtracker smartphone app:

$y=a+b * \log (x)+c x$

with $\mathrm{a}=3.19, \mathrm{~b}=0.71$ and $\mathrm{c}-0.001$. The time period between measurements (in days) is indicated by $x$. 
Measurement uncertainty and its effect on test variability

As described above, the critical difference is determined by 2 components: natural fluctuations (biological variation) and measurement uncertainty (analytical variation). Since biological variation is quite constant, the measurement uncertainty determines the amount of additional 'noise' that should be added to the variation in lab results.

The analytical variation is different for each laboratory result. It can be calculated that when the analytical variation is as great as the biological variation, the amount of additional noise that is added to the test result amounts to approximately $40 \%$ (5). If the measurement can be more precise and the analytical variation is threequarters of the biological variation, the additional noise falls to approximately $25 \%$. When the analytical variation is even smaller and is merely half of the biological variation, the analytical noise added to the biological variation is a mere $12 \%$. Within laboratory medicine, an analytical variation that is half of the biological variation is deemed acceptable. This ratio is an important quality criterion for the desired precision with which analyses should be performed (7). 


\section{References:}

1. Cheuvront SN, Fraser CG, Kenefick RW, Brett ER, Sawka MN. Reference change values for monitoring dehydration. Clin Chem Lab Med. 2011;49:1033-7.

2. Fraser, C.G. and Harris, C.G., Generation and application of data on biological variation in clinical chemistry. Crit Rev Clin Lab Sci, 1989. 27(5): p. 409-37

3. Ricós C, et al. Current databases on biological variation: pros, cons and progress. Scand J Clin Lab Invest. 1999;59:491-500.

4. HilderinkJM. Calculating the probability of change between two consecutive laboratory test results. [Reply to: Explaining laboratory test results to patients: what the clinician needs to know by Marice John O'Kane and Berenice Lopez]. BMJ. 2015;351:h5552.

5. Fraser C. Biological variation: from principles to practice. Washington, DC: AACC Press; 2001.

6. Labtracker. iTunes Appstore. https://appsto.re/nl/T3/r8.i

7. Ricós C, et al. Rationale for using data on biological variation. Clin Chem Lab Med. 2015;53:86370. Medline 


\section{Chapter 3}




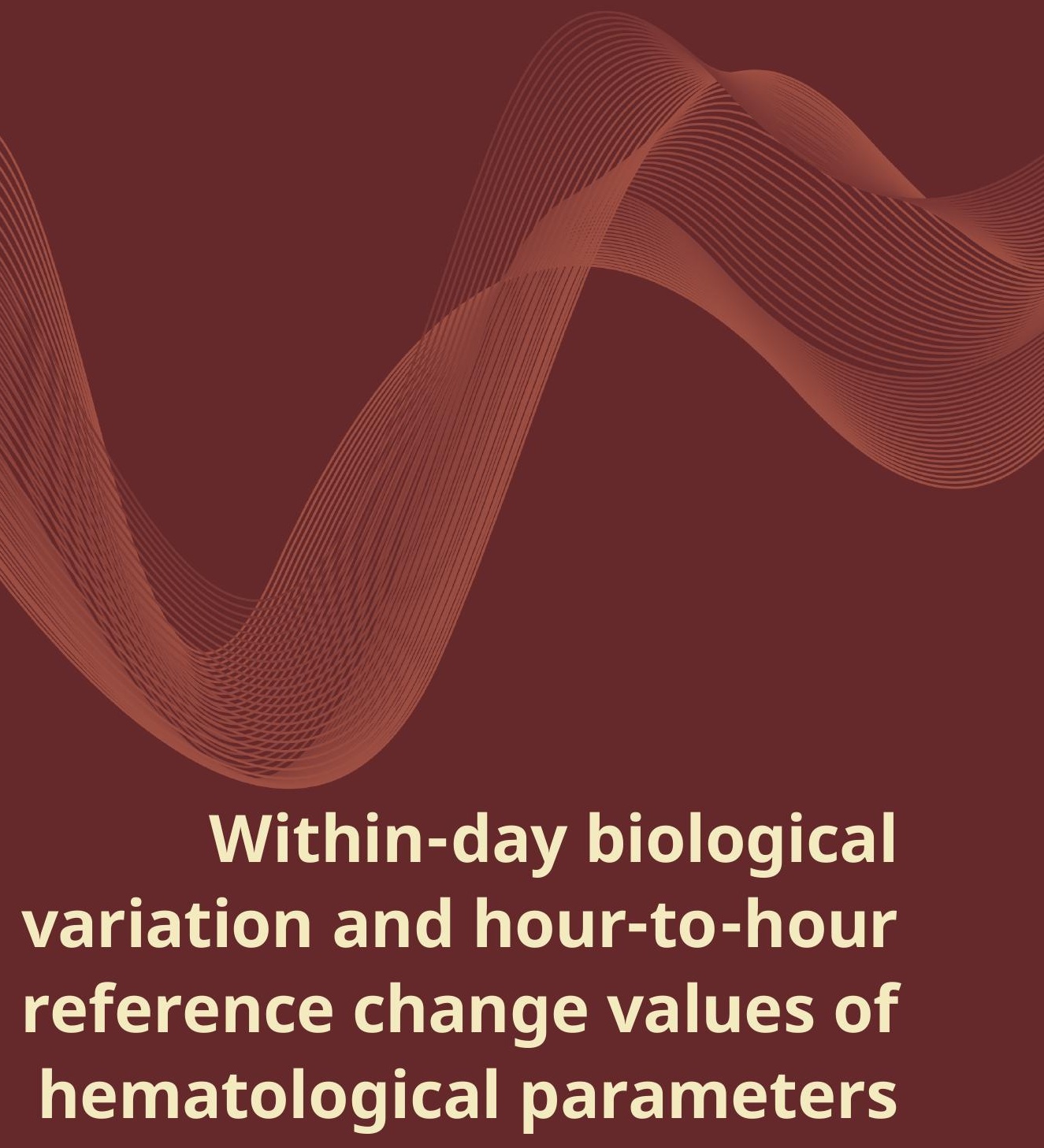

Judith M. Hilderink, Lieke J. J. Klinkenberg, Kristin M. Aakre, Norbert C.J. de Wit, Yvonne M.C. Henskens, Noreen van der Linden, Otto Bekers, Roger J.M.W. Rennenberg, Richard P. Koopmans, Steven J. R. Meex 


\begin{abstract}
Background

Middle and long-term biological variation data for hematological parameters have been reported in the literature. Within-day 24-hour variability profiles for hematological parameters are currently lacking. However, comprehensive hour-to-hour variability data is critical to detect diurnal cyclical rhythms, and to take into account the 'time of sample-collection' as a possible determinant of natural fluctuation. In this study, we assessed 24-hour variation profiles for 20 hematological parameters.
\end{abstract}

\title{
Method
}

Blood samples were collected under standardized conditions from 24 subjects every hour for 24 hours. At each measurement, 20 hematological parameters were determined in duplicate. Analytical variation, within-subject biological variation, between-subject biological variation, index of individuality and reference change values were calculated. For the parameters with a diurnal rhythm, hour-to-hour reference change values were determined.

\section{Results}

All parameters showed higher between-subject biological variation than withinsubject biological variation. Highest between-subject biological variation was found for eosinophils (46.6\% [95\% CI 34.9-70.1\%]) and the lowest value was mean corpuscular hemoglobin concentration (3.2\% [95\% CI 2.4-4.8\%]). Withinsubject biological variation varied from $0.4 \%$ [95\% CI $0.32-0.42 \%$ ] to $20.9 \%$ [95\% CI 19.4-22.6\%] for red cell distribution width and eosinophils, respectively. Six hematological parameters showed a diurnal rhythm.

\section{Conclusions}

We present complete 24 hour variability profiles for 20 hematological parameters. Hour-to-hour reference changes values may help to better discriminate between random fluctuations and true changes in parameters with rhythmic diurnal oscillations. 


\section{Background}

When interpreting changes in serial laboratory test results, health care professionals rely on their intuition and clinical experience to distinguish 'real changes' from physiological fluctuations. A more objective interpretation of changes in serial laboratory results can be achieved by calculating the 'reference change value' $(R C V)$, i.e. the required threshold for a change between serial measurements to be statistically significant (1). For a change to become significant, the difference between two measurements must exceed the total inherent variation. The calculated RCV can provide information to prevent random fluctuations from being interpreted as true changes.

Two factors can influence the RCV; the analytical variation $\left(C V_{A}\right)$ and the withinsubject biological variation $\left(\mathrm{CV}_{\mathrm{I}}\right)$. In laboratories that meet contemporary quality and precision specifications, $\mathrm{CV}_{\mathrm{I}}$ is the strongest determinant of test result variability (2). Studies of biological variation can provide insight in the natural fluctuations within and between subjects for a given parameter. Extensive biological variation studies are laborious and most studies of hematological parameters have only assessed biological variation between days (3-8). A few studies report withinday variation with a limited number of measurements (8-11). Complete 24-hour variability profiles for hematological parameters are currently lacking. Within day, hour-to-hour variability data is however critical to detect diurnal cyclical rhythms, and take into account the 'time of sample-collection' as a possible determinant of natural fluctuation. According to laboratory statistics from Maastricht University Medical Center, the assessment of repeated within-day hematological testing is common in hospitalized patients (up to $10 \%$ ).

The present study was conducted to provide comprehensive 24-hour variation profiles for 20 hematological parameters in 24 subjects. Specifically, within-day components variation and index of individuality were assessed. The index of individuality (II) is the ratio between the $\mathrm{CV}_{\mathrm{G}}$ and $\mathrm{CV}_{\mathrm{I}}$. It determines the utility of conventional population-based reference values (12). Finally, within-day RCVs were calculated for parameters with random variability over the day, whereas hour-tohour RCVs were calculated for hematological parameters with rhythmic oscillations during the day. 


\section{Materials and methods}

This study conforms to the principles of the Declaration of Helsinki and was approved by the institutional review board and the ethics committee at Maastricht University Medical Center. This study was registered at clinicaltrials.gov as NCT02091427. The current study meets the critical appraisal checklist criteria for studies of biological variation as proposed by Bartlett et al (13).

The routine hematological parameters we studied were: leukocytes, lymphocytes, neutrophils, monocytes, eosinophils, basophiles, erythrocytes, reticulocytes (percentage), reticulocytes (count), hemoglobin, hematocrit, mean corpuscular volume (MCV), mean corpuscular hemoglobin ( $\mathrm{MCH}$ ), mean corpuscular hemoglobin concentration (MCHC), red cell distribution width (RDW), mean platelet volume (MPV) and platelets. Research-hematological parameters included immature platelet fraction (IPF), plateletcrit (PCT) and platelet distribution width (PDW).

\begin{tabular}{|c|c|}
\hline \multicolumn{2}{|c|}{ Table 3.1. Participants' baseline characteristics } \\
\hline$n$ & 24 \\
\hline Age, years & $72 \pm 7$ \\
\hline Male, gender & $19(79 \%)$ \\
\hline $\mathrm{BMI}, \mathrm{kg} / \mathrm{m}^{2}$ & $27 \pm 5$ \\
\hline Systolic blood pressure, $\mathrm{mm} \mathrm{Hg}$ & $140 \pm 15$ \\
\hline Diastolic blood pressure, $\mathrm{mm} \mathrm{Hg}$ & $68 \pm 8$ \\
\hline Anti-hypertensive medication ${ }^{a}$ & $10(42 \%)$ \\
\hline Type 2 Diabetes Mellitus ${ }^{b}$ & $7(29 \%)$ \\
\hline Fasting glucose $c, \mathrm{mmol} / \mathrm{L}$ & $5.9(5.2-6.7)$ \\
\hline $\mathrm{HbA} 1 \mathrm{c}^{c}, \%$ & $5.7(5.3-6.5)$ \\
\hline Oral blood glucose lowering medication ${ }^{a}$ & $7(29 \%)$ \\
\hline Total cholesterol c, mmol/L & $3.7(4.8-5.4)$ \\
\hline HDL cholesterol ${ }^{c}, \mathrm{mmol} / \mathrm{L}$ & $1.3(1.6-1.9)$ \\
\hline LDL cholesterol c, $\mathrm{mmol} / \mathrm{L}$ & $1.7(2.5-3.0)$ \\
\hline Triglycerides c, mmol/L & $1.2(0.8-1.8)$ \\
\hline Lipid lowering medication a & $12(50 \%)$ \\
\hline eGFR ${ }^{c, d}, \mathrm{ml} / \mathrm{min} / 1.73 \mathrm{~m}^{2}$ & $75(61-92)$ \\
\hline
\end{tabular}

Data is presented as $n(\%)$, mean \pm SD or median (IQR). BMI, body mass index, eGFR, estimated glomerular filtration rate. ${ }^{a}$ Medication status was stable for at least three months prior to inclusion, and continued as normal during the entire study period, including the test-days. ${ }^{b}$ The diagnosis type 2 Diabetes Mellitus was defined as fasting plasma glucose $\geq 7.0 \mathrm{mmol} / \mathrm{L}$ and $/$ or $\mathrm{HbA} 1 \mathrm{c} \geq 6.5 \%$ ( $=48 \mathrm{mmol} / \mathrm{mol}$ ) (14) ` Value at 8:30 a.m. ${ }^{d}$ According to CKD-EPI Creatinine-cystatin C equation (15) 


\section{Study population}

The study population consisted of 24 volunteers ( 19 males and 5 females). These subjects were previously described in the study of Klinkenberg et al. (16). All participants provided written informed consent. The median age was 72 years (range 65-79 years). Baseline characteristics of the study population are shown in table 3.1. Exclusion criteria were: a change in medication use in the three months prior to the inclusion and a history of severe cardiovascular problems (acute myocardial infarction or stroke) within 12 months prior to inclusion. All participants were free from acute diseases at the time of the study.

\section{Study design}

After an overnight fast, participants arrived at 8 a.m. at the laboratory. Participants were asked to refrain from heavy physical labor and exercise training two days before the test day. They were sampled every hour by using an indwelling venous cannula in an antecubital vein during a time span of twenty-five hours. The participants spent the day seated in a chair or couch.

Standardized meals were consumed at 8:30 a.m. (breakfast), 12:30 p.m. (lunch) and 6:00 p.m. (dinner). The participants went to bed at 11:30 p.m. and got up the next morning at 7:00 a.m. At night, all subjects received a polyethylene coiled extending line (Vygon) that was attached to the indwelling venous catheter in order to obtain the blood samples and to minimize sleep disturbance.

\section{Laboratory measurements}

Blood sampling was performed under standardized conditions in order to minimize sources of pre-analytical variation. Blood samples were collected in ethylenediaminetraacetic acid (EDTA)-containing tubes ( $4 \mathrm{ml}$ ) every hour over a time span of 25 hours. Within 1 hour after collection, samples were analyzed in duplicate on a Sysmex XE-5000 analyzer (Sysmex Corporation, Kobe, Japan).

\section{Statistical analysis}

The outlier method of Burnett was used to check for analytical outliers (17). As suggested by Fraser and Harris (18), we tested for homogeneity in the analyticaland within-subject biological variances using the method described by Cochran. Briefly, analytical variances were tested for homogeneity by examining the ratio of the maximum variance to the sum of the variances and compare this with a critical 
value $(19,20)$. Subjects were excluded until homogeneity of variance was achieved (data not shown) (21). Finally, the criteria of Reed were used to identify betweensubject outliers $(20,22,23)$.

Between-subject- $\left(\mathrm{CV}_{\mathrm{G}}\right)$, within-subject biological variation $\left(\mathrm{CV}_{\mathrm{I}}\right)$, and analytical variation $\left(\mathrm{CV}_{\mathrm{A}}\right)$ were calculated by using a balanced analysis of variance (ANOVA) with a nested random design in two levels. Verified assumptions for using this model include a normally distributed error term of the means and constant variances (24). 95\% confidence intervals (CI) of these variance components were calculated using the method described by Burdick and Graybill $(24,25)$. The index of individuality (II) was calculated by the method described by Petersen et al. (26) and the RCVs were calculated according to the method of Fraser and Harris $(18,27)$. The residuals of the parameters followed a Gaussian distribution on the analytical and within-subject level. Therefore, no transformation into the natural logarithm was required.

$$
\begin{gathered}
\mathrm{II}=\frac{\sqrt{C V_{A}^{2}+C V_{I}^{2}}}{C V_{G}} \\
R C V=\sqrt{2} * Z * \sqrt{C V_{A}^{2}+C V_{I}^{2}}
\end{gathered}
$$

In the RCV formula, $Z$ represents the number of standard deviation appropriate for the desired level of statistical significance for a bidirectional change (e.g. 1.96 for $p=0.05$ and 2.58 for $p=0.01$ (3). For the RCV-calculations in this study, a Z-score of 1.96 was used.

In parameters with rhythmic diurnal variation, the assumption of constant variances is violated, precluding the calculation of an 'overall' within-day RCV. Instead, hourto hour RCVs were calculated for parameters with rhythmic diurnal oscillations, taking into account the systematic increase or decrease at all time-points $(18,28,29)$. Briefly, the average systematic change for each time point (as a percentage from the baseline value) was subtracted from the original individual data. Hence, variation due to rhythmic diurnal oscillations was eliminated and an artificial 'steady state' concentration with random fluctuation over the day was created.

With these adjusted data, assumptions for ANOVA were fulfilled, and components of biological variation could be calculated. Finally, the percentage hourly changes $(\beta)$ were added again to the individual time points. To calculate adjusted asymmetric 
RCVs that take into account rhythmic diurnal oscillations, the following formulae were used (28):

$$
\begin{aligned}
& R C V_{\text {upper limit }}=\beta+\sqrt{2} * 1.96 * \sqrt{C V_{A}^{2}+C V_{I}^{2}} \\
& R C V_{\text {lower limit }}=\beta-\sqrt{2} * 1.96 * \sqrt{C V_{A}^{2}+C V_{I}^{2}}
\end{aligned}
$$

All statistical calculations were performed using SPSS version 20 (IBM SPSS Statistics, IBM Corporation, Armonk, New York) and Matrix Laboratory (Matlab \& Simulink Student Suite R2015b).

Figure 3.1 (A-C): Subject-specific concentrations over $24 \mathrm{~h}$ with hourly sampling. Parameters were shown as absolute ranges (min-max).
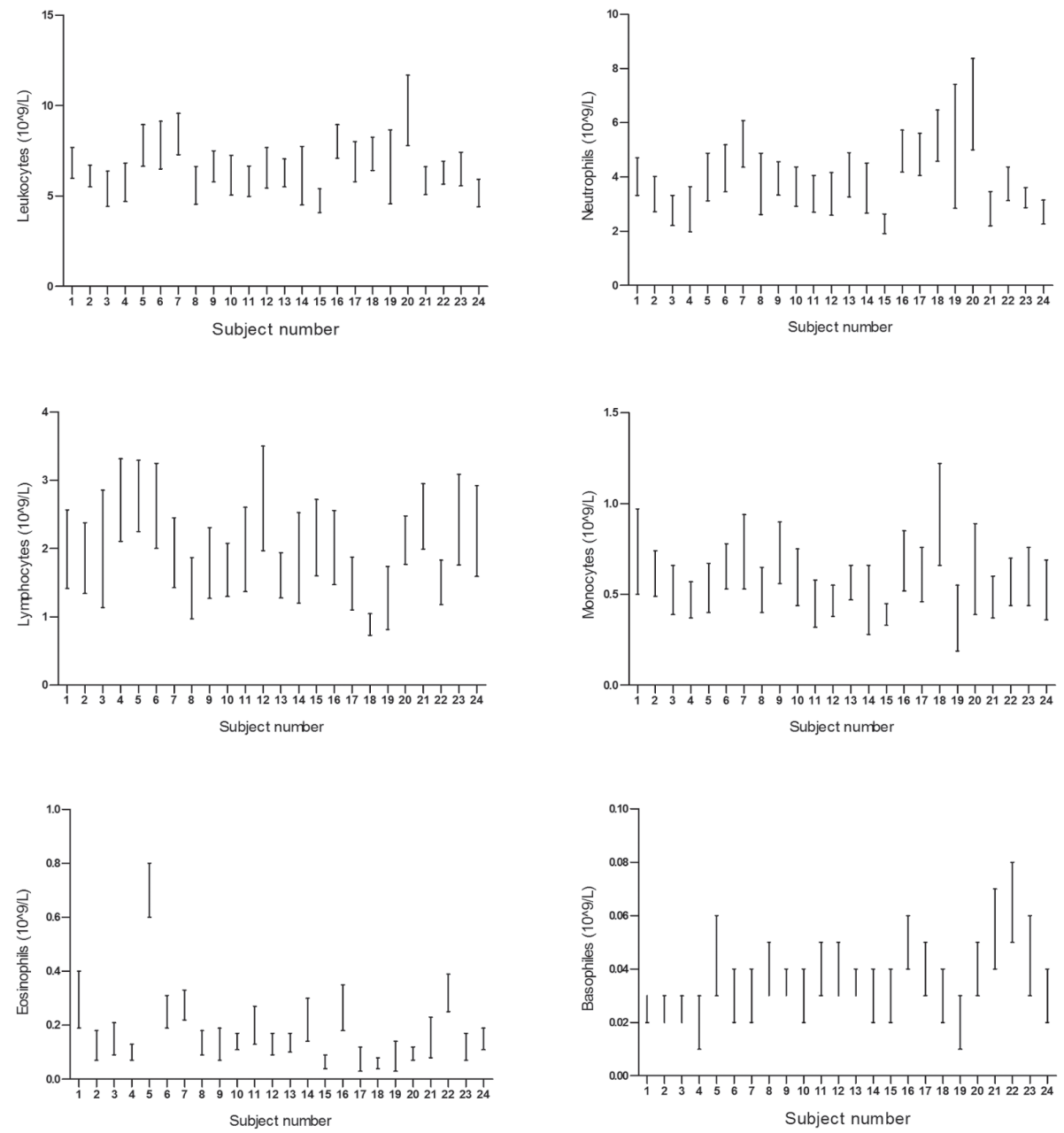
Figure 3.1 (continued)
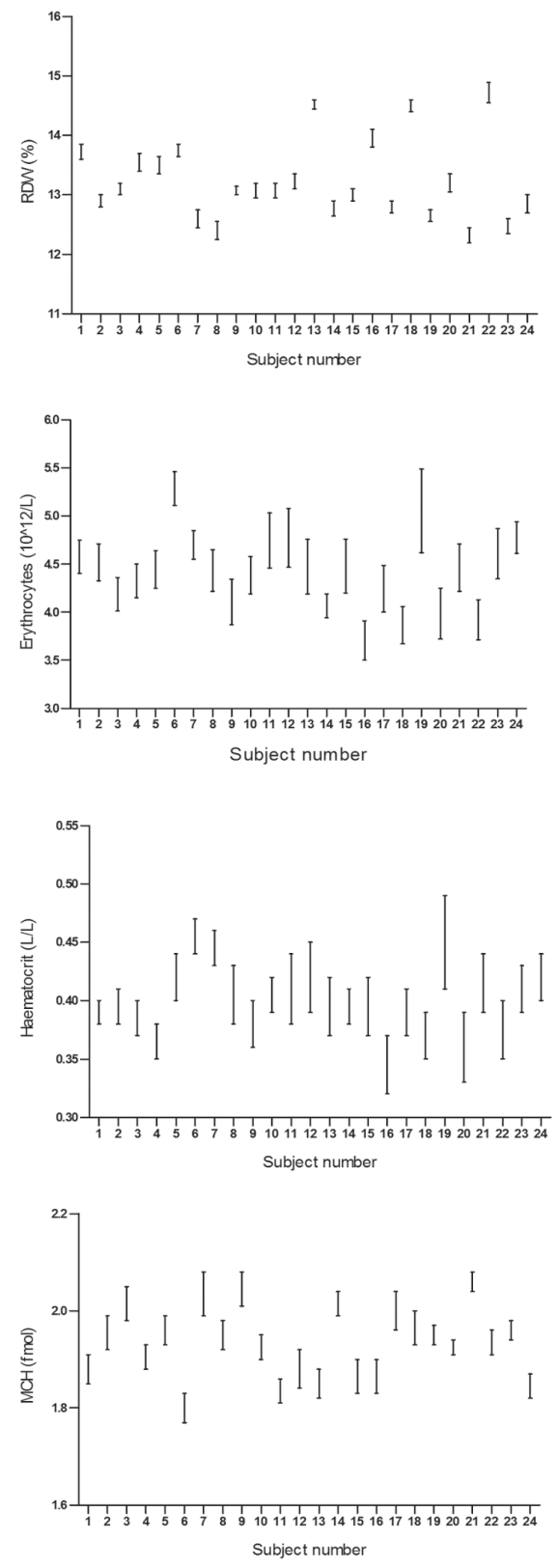
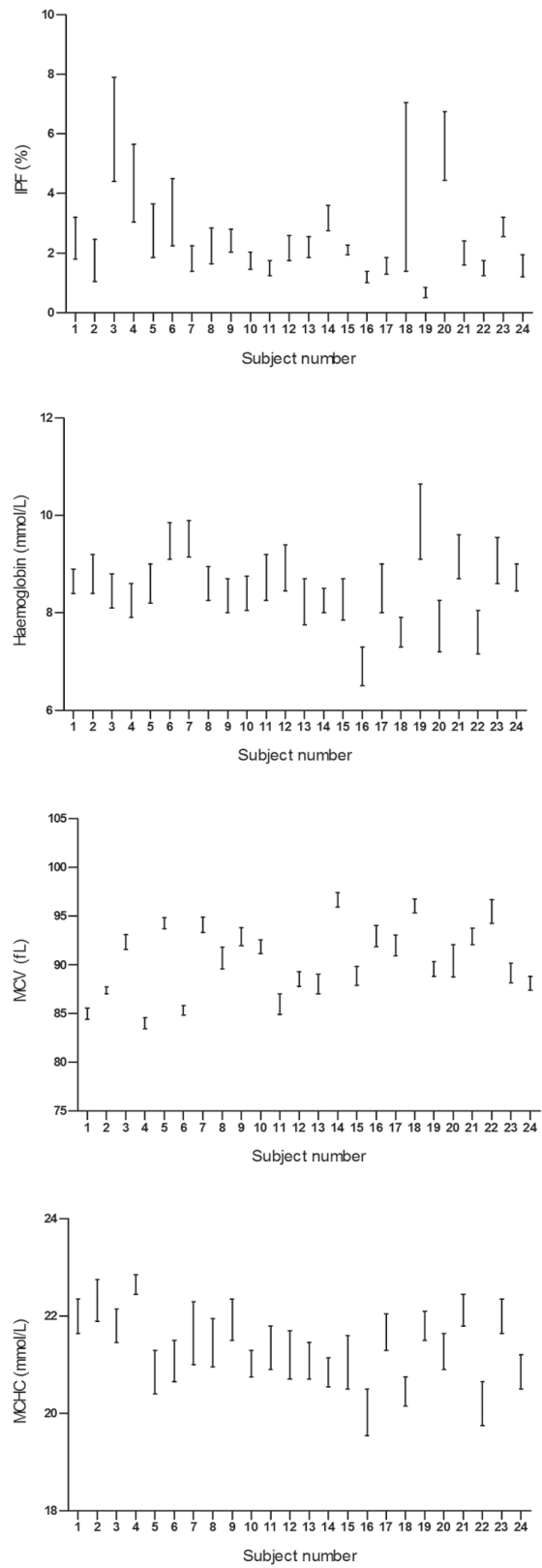
Figure 3.1 (continued)
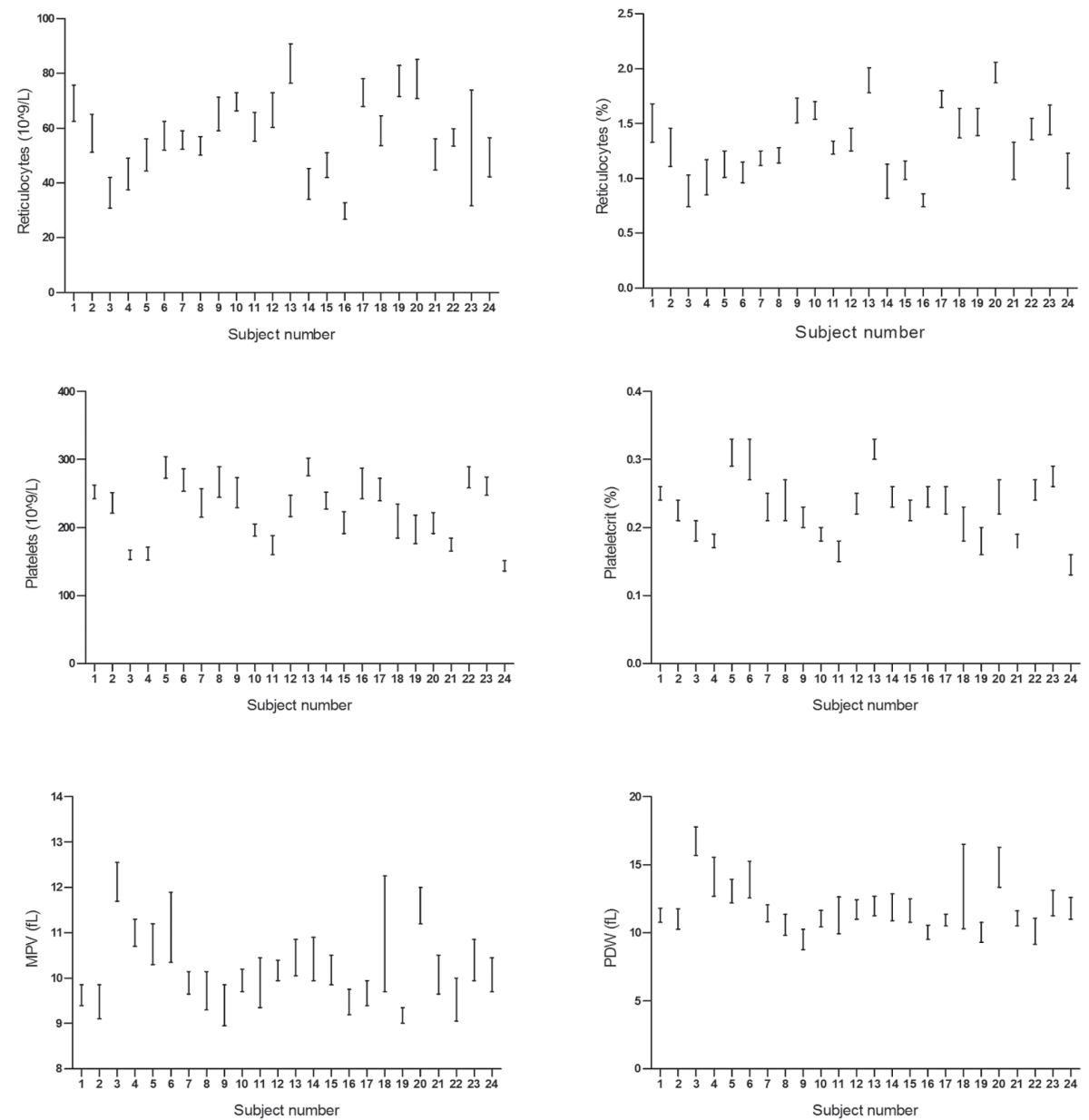

\section{Results}

Sample collection was incomplete for four subjects. Two subjects left the study prematurely, with respectively 12 and 4 hours of consecutive missing data (subject 1 and subject 8 . Since the missing values were not 'at random', a balanced design was required (30). In order to maintain a balanced design, in line with the statistical conditions for a balanced ANOVA with a nested random design in two levels, these subjects were excluded from all analyses. In two other individuals (subjects 15 and 23, night samples were missing due to technical problems with the intravenous cannula. Analyses in these subjects were restricted to the samples obtained 
between 8:30 a.m. and 11:30 p.m. Subject 20 became sick during the test day and due to a lack of a stable situation, this subject was excluded from all analyses.

Outliers and excluded subjects per parameter are listed in supplemental table 3.1. Concentration ranges of all hematological parameters are shown in figure 3.1.

\section{Posture-dependent changes of hematological parameters}

Concentrations of 7 parameters (hemoglobin, hematocrit, erythrocytes, reticulocytes, platelets and plateletcrit) were posture-dependent with an acute concentration drop at 11:30 p.m. (during transition from upright position to sleep position) and a prominent rise at 7 a.m. (change from sleep to upright position) (31). Figure 3.1 shows the average concentrations during the day of the parameters with posture-dependent changes. For these parameters, variation components were analyzed separately for the day and night. Since biological variation within both timeframes was similar (data not shown), only day- variation components (08:30 a.m. - 11:30 p.m. and 07:30 a.m. the next morning) are listed in table 3.2 for these parameters.

\section{4-hour variation components}

Variation components $\left(\mathrm{CV}_{\mathrm{A}^{\prime}} \mathrm{CV}_{\mathrm{I}^{\prime}}\right.$, and $\left.\mathrm{CV}_{\mathrm{G}}\right)$, indexes of individuality and RCVs are listed in table 3.2. The uncertainties regarding the calculations are reflected in the $95 \%$ confidence intervals (CI) given for all CV values $(24,25)$.

All parameters showed higher $\mathrm{CV}_{\mathrm{G}}$ than $\mathrm{CV}_{\mathrm{I}}$ values. Highest $\mathrm{CV}_{\mathrm{G}}$ was found for eosinophils (46.6\% [95\% CI 34.9-70.1\%]) and the lowest value was MCHC (3.2\%[95\% CI $2.4-4.8 \%]$ ). $\mathrm{CV}_{\mathrm{I}}$ was much lower and varied from $0.4 \%$ [95\% CI $0.32-0.42 \%$ ] to 20.9\% [95\% CI 19.4-22.6\%] of red cell distribution width (RDW) and eosinophils, respectively. Duplicate measurements of all parameters offered the advantage of analytical variation estimation at the same level as subject samples (20), and yielded an analytical variation range from $0.17 \%$ [95\% CI $0.16-0.18 \%$ ] to $21.2 \%$ [95\% CI 19.9-22.7\%] (of MCV and basophils, respectively). 
Figure 3.2: Diurnal variation of posture-dependent parameters.
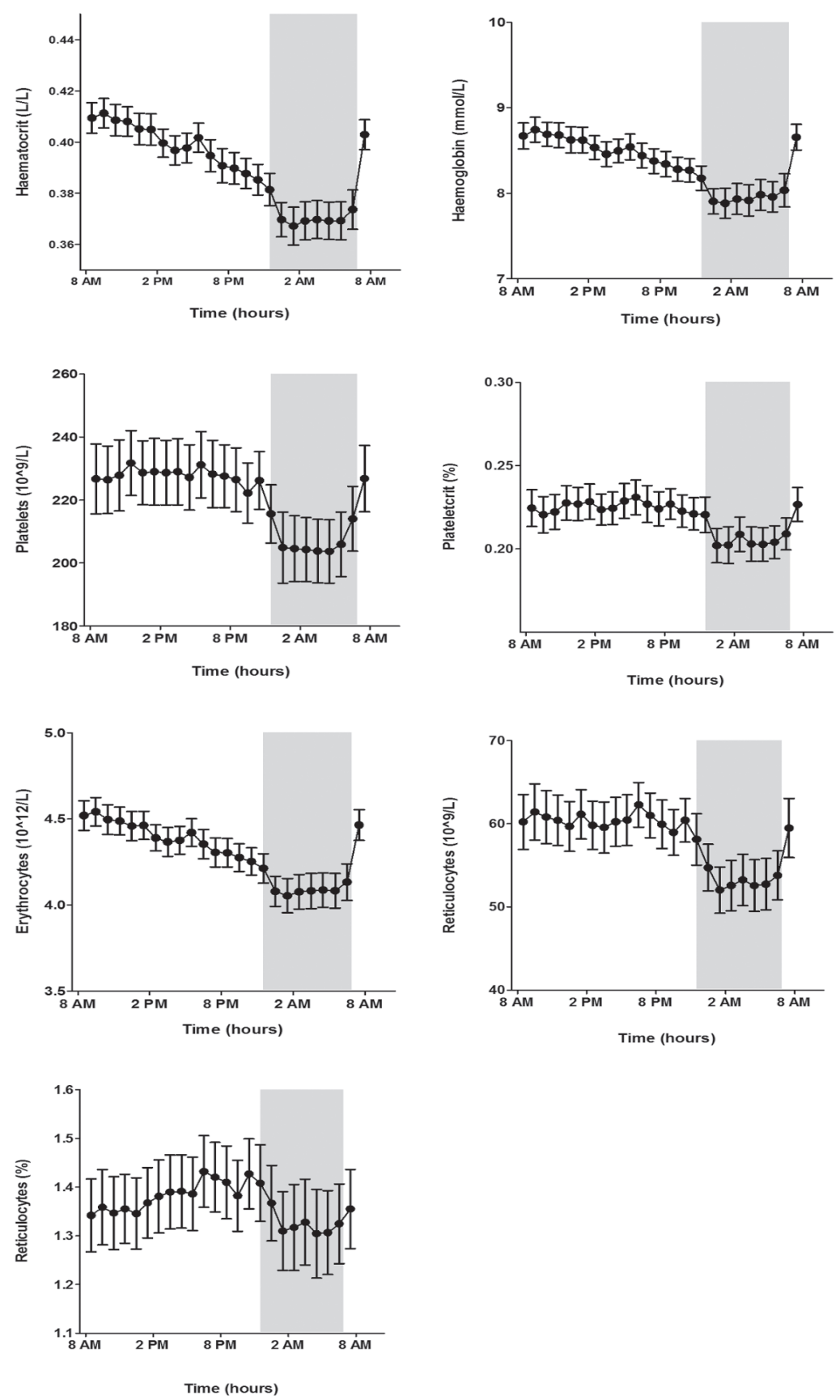

Values represent means \pm SEM of posture-dependent parameters. At 11:30 PM, there was a transition from upright position to sleep position and at 07:00 AM, posture changed from sleep to upright position 
Table 3.2. Analytical variation, biological variation, indexes of individuality and reference change values for hematological parameters

\begin{tabular}{|c|c|c|c|c|c|c|c|c|}
\hline & $n$ & $\begin{array}{l}\text { Measured } \\
\text { unit }\end{array}$ & Mean & $\mathrm{CV}_{\mathrm{G}}(\%)$ & $\mathrm{CV}_{\mathrm{I}}(\%)$ & $\mathrm{CV}_{\mathrm{A}}{ }^{\mathrm{a}}(\%)$ & $\begin{array}{r}\mathrm{RCV}_{95 \%} \\
(\%)\end{array}$ & II \\
\hline \multicolumn{9}{|c|}{ Routine hematological parameters } \\
\hline Leukocytes & 17 & $10 \wedge 9 / L$ & 6.6 & $13.7(10.2-21.0)$ & $7.0(6.6-7.6)$ & $1.4(1.3-1.5)$ & * & 0.5 \\
\hline Neutrophils & 18 & $10^{\wedge} 9 / \mathrm{L}$ & 3.8 & $22.3(16.7-33.5)$ & $11.1(10.3-11.9)$ & $1.9(1.8-2.0)$ & * & 0.5 \\
\hline Lymphocytes & 18 & $10 \wedge 9 / L$ & 1.9 & $26.0(19.4-36.1)$ & $15.0(14.1-16.1)$ & $2.7(2.5-2.9)$ & * & 0.6 \\
\hline Eosinophils & 18 & $10^{\wedge} 9 / \mathrm{L}$ & 0.2 & $46.6(34.9-70.1)$ & $20.9(19.4-22.6)$ & $9.3(8.7-9.9)$ & * & 0.5 \\
\hline Basophils & 19 & $10 \wedge 9 / L$ & 0.03 & $32.2(24.2-47.7)$ & $8.8(5.4-11.4)$ & $21.2(19.9-22.7)$ & * & 0.7 \\
\hline Monocytes & 18 & $10^{\wedge} 9 / \mathrm{L}$ & 0.6 & $16.8(12.5-25.3)$ & $12.3(11.4-13.3)$ & $6.2(5.8-6.6)$ & * & 0.8 \\
\hline Erythrocytes & 20 & $10^{\wedge} 12 / \mathrm{L}$ & 4.4 & $8.1(6.1-11.8)$ & $2.9(2.7-3.1)$ & $0.65(0.61-0.71)$ & 8.2 & 0.4 \\
\hline $\begin{array}{l}\text { Reticulocytes, } \\
\text { count }^{\mathrm{b}}\end{array}$ & 21 & $10^{\wedge} 9 / \mathrm{L}$ & 57.2 & $23.5(17.9-33.9)$ & $5.1(4.5-5.7)$ & $4.7(4.4-5.0)$ & 19.2 & 0.3 \\
\hline $\begin{array}{l}\text { Reticulocytes, } \\
\text { percentage }^{\text {b }}\end{array}$ & 20 & $\%$ & 1.3 & $23.3(17.7-34.1)$ & $4.0(3.5-4.6)$ & $4.6(4.3-4.9)$ & 16.9 & 0.3 \\
\hline Hemoglobin ${ }^{b}$ & 20 & $\mathrm{mmol} / \mathrm{L}$ & 8.5 & $7.2(5.4-10.5)$ & $2.7(2.5-2.9)$ & $0.55(0.51-0.59)$ & 7.6 & 0.4 \\
\hline Hematocrit ${ }^{\mathrm{b}}$ & 17 & $\mathrm{~L} / \mathrm{L}$ & 0.4 & $4.1(3.0-6.3)$ & $3.0(2.8-3.3))$ & $0.6(0.55-0.65)$ & 8.5 & 0.8 \\
\hline MCV & 17 & $\mathrm{fL}$ & 90.7 & $4.1(3.1-6.3)$ & $0.45(0.42-0.48)$ & $0.17(0.16-0.18)$ & 1.3 & 0.1 \\
\hline $\mathrm{MCH}$ & 19 & fmol & 1.9 & $4.0(3.0-5.9)$ & $0.8(0.78-0.89)$ & $0.17(0.16-0.17)$ & 2.4 & 0.2 \\
\hline $\mathrm{MCHC}$ & 19 & $\mathrm{mmol} / \mathrm{L}$ & 21.3 & $3.2(2.4-4.8)$ & $0.8(0.7-0.9)$ & $0.8(0.77-0.88)$ & 3.2 & 0.4 \\
\hline MPV & 18 & $\mathrm{fL}$ & 10.1 & $7.2(5.4-10.8)$ & $1.9(1.7-2.1)$ & $1.4(1.3-1.5)$ & 6.4 & 0.3 \\
\hline RDW & 17 & $\%$ & 13.2 & $4.2(3.1-6.3)$ & $0.37(0.32-0.42)$ & $0.47(0.44-0.50)$ & 1.7 & 0.1 \\
\hline Platelets ${ }^{b}$ & 19 & $10 \wedge 9 / L$ & 228 & $20.2(15.2-29.8)$ & $3.2(2.9-3.5)$ & $1.5(1.4-1.6)$ & 9.8 & 0.2 \\
\hline \multicolumn{9}{|c|}{ Research hematological parameters } \\
\hline IPF & 17 & $\%$ & 2.1 & $42.3(31.4-64.5)$ & $12.8(11.6-14.0)$ & $10.2(9.5-10.9)$ & 45.2 & 0.4 \\
\hline Plateletcrit ${ }^{\mathrm{b}}$ & 16 & $\%$ & 0.2 & $20.1(14.9-31.2)$ & $3.5(3.1-4.0)$ & $2.8(2.6-3.1)$ & 12.6 & 0.2 \\
\hline PDW & 18 & $\mathrm{fL}$ & 11.6 & $14.6(10.9-21.9)$ & $3.7(3.2-4.2)$ & $3.8(3.5-4.2)$ & 14.7 & 0.4 \\
\hline
\end{tabular}

$95 \%$ confidence intervals are shown in brackets. ${ }^{a}$ on the basis of duplicate measurements. ${ }^{\mathrm{b}}$ This parameter has a diurnal rhythm and therefore, hour-to-hour RCVs are calculated (see Table 3 and Supplemental Table 1). ' Concentrations are posture-dependent, hence only data from 08:30 a.m. - 11:30 p.m. and 07:30 a.m. were used. $n$ number of subjects after removing outliers. CV, coefficient of variation, $\mathrm{CV}_{\mathrm{G}^{\prime}}$, between-subject biological variation, $\mathrm{CV}_{\mathrm{I}^{\prime}}$ within-subject biological variation, $\mathrm{CV}_{\mathrm{A}^{\prime}}$ analytical variation, $\mathrm{II}$, index of individuality, $\mathrm{RCV}$, reference change value, $\mathrm{MCV}$, mean corpuscular volume, $\mathrm{MCH}$, mean corpuscular haemoglobin, MCHC, mean corpuscular haemoglobin concentration, MPV, mean platelet volume, RDW, red cell distribution width, IPF, immature platelet fraction, PDW, platelet distribution width 
All parameters, except hematocrit, basophils and monocytes, showed marked individuality, with individuality indexes $<0.6$. The lowest calculated individuality index was 0.1 for MCV and RDW and the highest calculated index of individuality was 0.8 for hematocrit and monocytes. The RCVs ranged from 1.3\% (MCV) to $45.2 \%$ (IPF).

\section{Rhythmic diurnal variation in hematological parameters}

Six hematological parameters showed a diurnal rhythm unrelated to posturechanges in blood volume: leukocytes, neutrophils, lymphocytes, eosinophils, basophils and monocytes. These intrinsic diurnal rhythms were previously described by Druzd et al. (32), Lasselin et al. (33) and Sennels et al. (10).

Rhythmic diurnal oscillations precluded calculation of overall variation components and RCVs. Instead, hour-to-hour RCVs were calculated, which take into account the structural change according to the diurnal rhythm. Hour-to-hour RCVs facilitate accurate interpretations of changes between two serial laboratory measurements within 24 hours, taking into account the rhythmic diurnal oscillations. Hour-tohour RCVs of neutrophils range from $-41 \%$ to $46 \%$, depending on the time points chosen (table 3.3). Hour-to-hour RCVs for other parameters with a diurnal rhythm are presented in supplemental tables 3.2A-E. 
Table 3.3. Hour-to-hour RCVs of Neutrophils

Neutrophils

(10^9/L)

\begin{tabular}{|c|c|c|c|c|c|c|c|c|c|c|c|}
\hline $\begin{array}{l}\text { Second } \\
\text { measure- } \\
\text { ment } \rightarrow\end{array}$ & $\begin{array}{c}08: 30 \\
\text { AM }\end{array}$ & $\begin{array}{c}09: 30 \\
\text { AM }\end{array}$ & $\begin{array}{c}10: 30 \\
\text { AM }\end{array}$ & $\begin{array}{c}11: 30 \\
\text { AM }\end{array}$ & $\begin{array}{c}12: 30 \\
\text { AM }\end{array}$ & $\begin{array}{c}\text { 01:30 } \\
\text { PM }\end{array}$ & $\begin{array}{c}\text { 02:30 } \\
\text { PM }\end{array}$ & $\begin{array}{c}\text { 03:30 } \\
\text { PM }\end{array}$ & $\begin{array}{c}04: 30 \\
\text { PM }\end{array}$ & $\begin{array}{c}05: 30 \\
\text { PM }\end{array}$ & $\begin{array}{c}06: 30 \\
\text { PM }\end{array}$ \\
\hline
\end{tabular}

\section{First mea-}

surement $\downarrow$

\begin{tabular}{|c|c|c|c|c|c|c|c|c|c|c|}
\hline 08:30 AM & -1237 & -742 & -545 & -445 & -346 & -742 & -841 & -1039 & -1435 & -1535 \\
\hline 09:30 AM & & -2029 & -1831 & -1732 & -1733 & -2029 & -2128 & -2326 & -2623 & -2722 \\
\hline 10:30 AM & & & $-22 \quad 27$ & -2228 & -2128 & -2425 & -2624 & -2722 & -3019 & -3118 \\
\hline 11:30 AM & & & & -2425 & -2326 & -2623 & -2822 & -2920 & -3217 & -3316 \\
\hline 12:30 AM & & & & & -2425 & -2722 & -2821 & -3019 & -3316 & -3416 \\
\hline 01:30 PM & & & & & & -2822 & -2920 & -3019 & -3316 & -3415 \\
\hline 02:30 PM & & & & & & & -2623 & -2722 & -3119 & -3118 \\
\hline 03:30 PM & & & & & & & & -2623 & -2920 & -3019 \\
\hline 04:30 PM & & & & & & & & & -2821 & -2921 \\
\hline 05:30 PM & & & & & & & & & & -2524 \\
\hline 06:30 PM & & & & & & & & & & \\
\hline 07:30 PM & & & & & & & & & & \\
\hline 08:30 PM & & & & & & & & & & \\
\hline 09:30 PM & & & & & & & & & & \\
\hline 10:30 PM & & & & & & & & & & \\
\hline 11:30 PM & & & & & & & & & & \\
\hline 12:30 PM & & & & & & & & & & \\
\hline 01:30 AM & & & & & & & & & & \\
\hline 02:30 AM & & & & & & & & & & \\
\hline 03:30 AM & & & & & & & & & & \\
\hline 04:30 AM & & & & & & & & & & \\
\hline 05:30 AM & & & & & & & & & & \\
\hline 06:30 AM & & & & & & & & & & \\
\hline 07:30 AM & & & & & & & & & & \\
\hline
\end{tabular}

The vertical column corresponds to the time of the first measurement and the horizontal row corresponds to the time of the second measurement. Each box indicates the percentage change range that is considered 'normal' $(95 \% \mathrm{CI})$, relative to the first measurement. If a percentage change between two consecutive measurements is outside this range, the change can be considered statistically significant. 


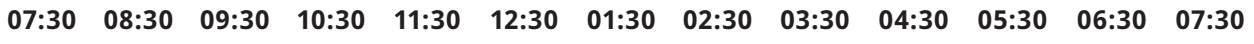

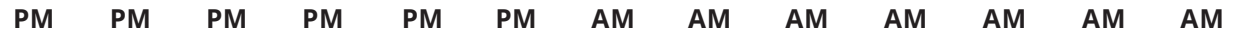

\begin{tabular}{|c|c|c|c|c|c|c|c|c|c|c|c|c|}
\hline-1435 & -1435 & -1732 & -1831 & -1831 & -2227 & -1831 & -1930 & -2227 & -2426 & -2227 & -2227 & -1139 \\
\hline-2623 & -2623 & -2920 & -3020 & -3020 & -3416 & -3020 & -3119 & -3316 & -3515 & -3416 & -3316 & -2326 \\
\hline-3119 & -3019 & -3316 & -3416 & -3416 & -3812 & -3416 & -3515 & -3712 & -3911 & -3712 & -3712 & -2822 \\
\hline-3317 & -3217 & -3514 & -3614 & -3614 & -3910 & -3614 & -3613 & -3910 & -409 & -3910 & -3910 & -3020 \\
\hline-3316 & -3316 & -3514 & -3613 & -3613 & -409 & -3613 & -3712 & -4010 & -418 & -409 & -4010 & -3019 \\
\hline-3416 & -3316 & -3613 & -3713 & -3713 & -409 & -3713 & -3812 & -409 & -418 & -409 & -409 & -3119 \\
\hline-3118 & -3119 & -3316 & -3415 & -3415 & -3811 & -3415 & -3514 & -3812 & -3910 & -3812 & -3812 & -2821 \\
\hline-3020 & -2920 & -3217 & -3317 & -3316 & -3713 & -3317 & -3416 & -3613 & -3812 & -3613 & -3613 & -2723 \\
\hline-2821 & -2821 & -3119 & -3118 & -3118 & -3514 & -3118 & -3217 & -3514 & -3613 & -3514 & -3514 & -2524 \\
\hline-2524 & -2525 & -2722 & -2821 & -2821 & -3217 & -2821 & -2920 & -3217 & -3316 & -3217 & -3217 & -2227 \\
\hline \multirow[t]{13}{*}{-2425} & -2425 & -2723 & -2822 & -2822 & -3218 & -2822 & -2921 & -3118 & -3317 & -3218 & -3118 & -2128 \\
\hline & -2425 & -2722 & -2821 & -2821 & -3217 & -2821 & -2920 & -3218 & -3316 & -3217 & -3218 & -2128 \\
\hline & & -2722 & -2821 & -2821 & -3217 & -2821 & -2920 & -3217 & -3316 & -3217 & -3217 & -2227 \\
\hline & & & -2524 & -2624 & -3019 & -2524 & -2723 & -2920 & -3118 & -3020 & -2920 & -1930 \\
\hline & & & & -2525 & -2920 & -2525 & -2624 & -2921 & -3019 & -2921 & -2921 & -1831 \\
\hline & & & & & -2920 & -2525 & -2624 & -2821 & -3019 & -2921 & -2821 & -1831 \\
\hline & & & & & & -2029 & -2128 & -2425 & -2624 & -2425 & -2425 & -1336 \\
\hline & & & & & & & -2624 & -2821 & -3019 & -2921 & -2921 & -1831 \\
\hline & & & & & & & & -2722 & -2920 & -2822 & -2822 & -1732 \\
\hline & & & & & & & & & -2623 & -2524 & -2525 & -1436 \\
\hline & & & & & & & & & & -2326 & -2326 & -1237 \\
\hline & & & & & & & & & & & -2425 & -1336 \\
\hline & & & & & & & & & & & & -1436 \\
\hline
\end{tabular}


Physiological basis of rhythmic diurnal variation

Rhythmic oscillations of parameters can be either intrinsic or in response to circadian variation of other parameters such as cortisol. As an illustration, we measured cortisol in all subjects of this study and plotted diurnal cortisol concentrations and lymphocytes together in supplemental figure 3.1. In this figure, exactly the opposite rhythmic oscillations are evident. This is in line with the established physiological effects of cortisol on lymphocyte levels $(34,35)$.

\section{Discussion}

This study presents novel 24-hour variability profiles for 20 hematological parameters. We have calculated variation components $\left(\mathrm{CV}_{\mathrm{A}^{\prime}} \mathrm{CV}_{\mathrm{I}}\right.$ and $\left.\mathrm{CV}_{\mathrm{G}}\right)$, indexes of individuality and RCVs of these parameters in order to better differentiate true changes from random fluctuations when interpreting changes between serial laboratory measurements within a day. Repeated within-day testing is customary in various clinical settings, e.g. the evaluation of therapeutic interventions, or when monitoring the disease course. The availability of comprehensive, 24hour variability data in the scientific literature represents a major gap for most laboratory parameters.

Comprehensive 24-hour sampling allowed to investigate diurnal cyclical rhythms and, if present, to take into account the 'time of sample-collection' as a determinant of natural fluctuation. For the six parameters with a detectable diurnal rhythm, we present for the first time hour-to hour RCVs that may help to objectively evaluate changes between any two time-points of the day.

\section{Hour-to-hour RCVs}

RCV values describe the maximum non- significant hour-to hour changes that might be seen in a 'steady state situation'. In a parameter with a diurnal rhythm, there is no steady state situation, because the level of the parameter changes randomly as well as systematically over time. If the systematic component is ignored, the $\mathrm{CV}_{\mathrm{I}}{ }^{\mathrm{S}} \mathrm{s}$ will be inflated and the RCVs will be systematically biased up or down depending on the hour. As a result, the RCVs will not properly reflect the bandwidth within which values are to be expected with a $95 \%$ probability.

To calculate unbiased RCVs, we calculated the $\beta$ value for each time point as a percentage from the first result. The data was then adjusted according to the 
$\beta$ value, and an adjusted steady state was achieved. After calculating the $C V_{I}$ 's and $C V_{A}{ }^{\prime} s$, the $\beta$ 's were combined with the $C V_{I}{ }^{\prime}$ s and $C V_{A}$ 's in order to get an asymmetrical hour-to-hour RCV with an upper-and lower limit. This method was first described by Aakre et al.(28).

By implementing the $\beta$ 's the way we did, we were able to fully correct for any variation in parameter levels during the day. The advantage of our approach is that it yields unbiased results, but the downside is that it requires a substantial number of betas to be estimated on the data, potentially increasing estimation error. Other approaches, such as fitting a (co)sinus function to the data, make stronger assumptions about the nature of the fluctuations in the parameter during the day, which allows them to be implemented with fewer betas to estimate and, accordingly, reduced estimation error. However, if the inherent assumptions about the nature of the fluctuations are not correct, they yield biased results. In this trade-off, we chose to avoid making additional assumptions and, with that, to avoid introducing bias, at the cost of estimating more betas. What approach is optimal in the context of diurnal parameter rhythms is to our knowledge still an open question, and may vary per parameter. However, for parameters such as neutrophils, any of these approaches is an improvement over the old assumption of a constant diurnal level.

The asymmetry of the calculated hour-to-hour RCVs in table 3.3 shows how different they can be from standard symmetrical RCVs and underlines the clinical importance of letting go of the restricting assumption of a constant diurnal level.

\section{Influence of fasting vs non-fasting}

To the best of our knowledge, fasting does not have a direct influence on the parameters that were studied. As all conditions including mealtimes were standardized, we cannot empirically prove that any fasting leaves our results unaffected.

\section{Comparison of $C V_{I}$ values to those in previous studies}

In general, the $\mathrm{CV}_{\mathrm{I}}$ values of this study were comparable to those in previous studies about biological variation of hematological parameters. Overall, other studies about within-day biological variation of hematological parameters found slightly higher $\mathrm{CV}_{\mathrm{I}}$ values, except for MCV and Platelet Distribution Width (PDW) $(9,10)$. However, when comparing the $\mathrm{CV}_{\mathrm{I}}$ values of the current study with previous studies 
about between-day biological variation, some parameters showed slightly lower $\mathrm{CV}_{\mathrm{I}}$ values in other studies. The study of Fraser et al. reported $\mathrm{CV}_{\mathrm{I}}$ values of 9.4 for lymphocytes, compared to $15.0(14.1$ - 16.1) in our study (36). A potential explanation for this difference could be that the time of sample collection was standardized in Fraser's study. Hereby, the intrinsic rhythmic oscillations during the day are not included in the $\mathrm{CV}_{\mathrm{I}}$ and therefore the $\mathrm{CV}_{\mathrm{I}}$ is lower.

\section{Gradual decrease of hemoglobin and hematocrit during the day}

A remarkable finding is the gradual decrease of hemoglobin, hematocrit and erythrocytes during the day, before the 'acute concentration drop' due to the transition from an upright to lying position in the evening. The other parameters that were posture-dependent did not show such a gradual decrease during the day. A dilution effect for the gradual decrease is unlikely since other parameters such as platelets are unaffected.

Hemoglobin concentration decreased from $8.2 \mathrm{mmol} / \mathrm{L}$ in sitting position to 7.9 $\mathrm{mmol} / \mathrm{L}$ in lying position $(-3.8 \%)$ and increased from $8.0 \mathrm{mmol} / \mathrm{L}$ to $8.7 \mathrm{mmol} / \mathrm{L}$ $(+8.8 \%)$ during the transition from a lying to an upright position. Parallel changes were found for hematocrit, $0.37 \mathrm{~L} / \mathrm{L}$ to $0.38 \mathrm{~L} / \mathrm{L}(-2.6 \%)$ and from $0.37 \mathrm{~L} / \mathrm{L}$ to 0.40 L/L (+8.1\%), respectively.

\section{Index of individuality}

Parameters with individuality indexes of $>1.4$ show very little individuality and, therefore, the use of reference values is considered appropriate because unusual values for almost all subjects will lie outside the reference limits $(18,26,37)$. For parameters with individuality indexes of $<0.6$ however, population based reference values have limited utility, and the use of RCVs may have added value. Analysis of the samples obtained from participants in this study revealed that all parameters, except hematocrit, basophils and monocytes, showed substantial individuality indexes below 0.6 (table 3.2). Consequently, for almost all of the studied parameters, calculated RCVs may offer substantial benefit when monitoring a patient within a day.

\section{Study Limitations}

Some limitations in the study population merit attention. We have included 16-21 subjects (depending on the parameter) in biological variation analyses. Although the number of excluded subjects was rather high for some parameters 
(i.e. plateletcrit, 8 subjects), 16 subject still afforded sufficient power to make reliable estimations for the various components of variation (24). However, due to disproportional inclusion of both sexes (men $n=19$, women $n=5$ ), we were unable to assess the appropriateness of partitioning $\mathrm{CV}_{\mathrm{I}}$ and $\mathrm{CV}_{\mathrm{G}}$ by sex. Besides, the age range of the subjects was 65-79 years. Although this age category corresponds to the age of the average hospitalized patient, the results of this study cannot be extrapolated to other age categories.

Another limitation that should be mentioned is that RCV calculations in this study were based on the $\mathrm{CV}_{\mathrm{A}}$ values we reported in table 3.2. However, $\mathrm{CV}_{\mathrm{A}}$ is to some degree concentration-dependent and RCVs may vary according to the concentration of the parameter. However, for the majority of the routine hematological parameters $(65 \%), \mathrm{CV}_{\mathrm{A}}$ was $\leq 1 / 2 \mathrm{CV}_{\mathrm{I}^{\prime}}$ which diminishes the contribution of $\mathrm{CV}_{\mathrm{A}}$ to the overall variation to less than $12 \%(20)$.

\section{Conclusion}

This is the first study that assesses the within-day biological variation of most of the relevant hematological parameters by using hourly blood samples. Moreover, it is the first to study the hour-to-hour fluctuations in parameter levels that occur for certain parameters during the day. It presents $\mathrm{CV}_{\mathrm{G}^{\prime}} \mathrm{CV}_{\mathrm{I}^{\prime}} \mathrm{CV}_{\mathrm{A}^{\prime}}$ indexes of individuality and RCVs for 20 hematological parameters, and where appropriate adjusts the RCVs for diurnal rhythms. 24-hour biological variation profiles are an important part of the scientific basis for better interpretation of serial within-day laboratory measurements. 


\section{References}

1. Fraser CG. Reference change values. Clin Chem Lab Med 2012;50:807-12.

2. Bailey D, Bevilacqua V, Colantonio DA, Pasic MD, Perumal N, Chan MK, Adeli K. Pediatric within-day biological variation and quality specifications for 38 biochemical markers in the caliper cohort. Clin Chem 2014;60:518-29.

3. Nunes LA, Brenzikofer R, de Macedo DV. Reference change values of blood analytes from physically active subjects. Eur J Appl Physiol 2010;110:191-8.

4. Maes M, Scharpe S, Cooreman W, Wauters A, Neels H, Verkerk R, et al. Components of biological, including seasonal, variation in hematological measurements and plasma fibrinogen concentrations in normal humans. Experientia 1995;51:141-9.

5. Dot D, Miro J, Fuentes-Arderiu X. Within-subject biological variation of hematological quantities and analytical goals. Arch Pathol Lab Med 1992;116:825-6.

6. Pineda-Tenor D, Laserna-Mendieta EJ, Timon-Zapata J, Rodelgo-Jimenez L, Ramos-Corral R, Recio-Montealegre A, Reus MG. Biological variation and reference change values of common clinical chemistry and haematologic laboratory analytes in the elderly population. Clin Chem Lab Med 2013;51:851-62.

7. Rudez G, Meijer P, Spronk HM, Leebeek FW, ten Cate H, Kluft C, de Maat MP. Biological variation in inflammatory and hemostatic markers. J Thromb Haemost 2009;7:1247-55.

8. Statland BE, Winkel P, Harris SC, Burdsall MJ, Saunders AM. Evaluation of biologic sources of variation of leukocyte counts and other hematologic quantities using very precise automated analyzers. Am J Clin Pathol 1978;69:48-54.

9. Yang D, Zhou Y, Yang C. Daytime biological variation of hematological parameters in a healthy chinese population. Int J Lab Hematol 2016.

10. Sennels HP, Jorgensen HL, Hansen AL, Goetze JP, Fahrenkrug J. Diurnal variation of hematology parameters in healthy young males: The bispebjerg study of diurnal variations. Scand J Clin Lab Invest 2011;71:532-41.

11. Zhang $P$, Tang $H$, Chen $K$, Chen $Y, X u$ D. Biological variations of hematologic parameters determined by unicel dxh 800 hematology analyzer. Arch Pathol Lab Med 2013;137:1106-10.

12. Harris EK. Statistical aspects of reference values in clinical pathology. Prog Clin Pathol 1981;8:45-66.

13. Bartlett WA, Braga F, Carobene A, Coskun A, Prusa R, Fernandez-Calle P, et al. A checklist for critical appraisal of studies of biological variation. Clin Chem Lab Med 2015;53:879-85.

14. American Diabetes A. Diagnosis and classification of diabetes mellitus. Diabetes Care 2004;27 Suppl 1:S5-S10.

15. Inker LA, Schmid CH, Tighiouart $\mathrm{H}$, Eckfeldt JH, Feldman HI, Greene T, et al. Estimating glomerular filtration rate from serum creatinine and cystatin c. N EnglJ Med 2012;367:20-9.

16. Klinkenberg LJJ, Wildi K, van der Linden N, Kouw IWK, Niens M, Twerenbold R, et al. A diurnal rhythm of cardiac troponin: Consequences for the diagnosis of acute myocardial infarction. Clin Chem (accepted) 2016.

17. Burnett RW. Accurate estimation of standard deviations for quantitative methods used in clinical chemistry. Clinical chemistry 1975;21:1935-8.

18. Fraser CG, Harris EK. Generation and application of data on biological variation in clinical chemistry. Critical reviews in clinical laboratory sciences 1989;27:409-37.

19. IUPAC. Protocol for the design, conduct and interpretation of method performance studies. Pure Appl Chem 1995;67.

20. Fraser CG. Biological variation: From principles to practice. AACC Press, Washington DC 2001. 
21. Carlsen S PP, Skeie S, Skadberg O, Sandberg S. . Within subject biological variation of glucose and hba1c in healthy persons and in type 1 diabetes patients. Clin Chem Lab Med 2011;49:1501-7.

22. Reed AH, Henry RJ, Mason WB. Influence of statistical method used on the resulting estimate of normal range. Clinical chemistry 1971;17:275-84.

23. Dixon WJ. Processing data for outliers. Biometrics 1953;9:74-89.

24. Roraas T, Petersen PH, Sandberg S. Confidence intervals and power calculations for withinperson biological variation: Effect of analytical imprecision, number of replicates, number of samples, and number of individuals. Clin Chem 2012;58:1306-13.

25. Burdick RK GF. Confidence intervals on variance components. New York, Marcel Dekker 1992.

26. Petersen PH, Fraser CG, Sandberg S, Goldschmidt H. The index of individuality is often a misinterpreted quantity characteristic. Clin Chem Lab Med 1999;37:655-61.

27. Harris EK, Yasaka T. On the calculation of a "reference change" for comparing two consecutive measurements. Clinical chemistry 1983;29:25-30.

28. Aakre KM, Roraas T, Petersen PH, Svarstad E, Sellevoll H, Skadberg O, et al. Weekly and 90-minute biological variations in cardiac troponin $\mathrm{t}$ and cardiac troponin i in hemodialysis patients and healthy controls. Clin Chem 2014;60:838-47.

29. Solvik UO, Petersen PH, Monsen G, Stavelin AV, Sandberg S. Discrepancies in international normalized ratio results between instruments: A model to split the variation into subcomponents. Clinical chemistry 2010;56:1618-26.

30. McDonald JH. Handbook of biological statistics (3rd edition). Sparky House Publishing, Baltimore, Maryland 2014.

31. Felding P, Tryding N, Hyltoft Petersen P, Horder M. Effects of posture on concentrations of blood constituents in healthy adults: Practical application of blood specimen collection procedures recommended by the scandinavian committee on reference values. Scand J Clin Lab Invest 1980;40:615-21.

32. Druzd D, de Juan A, Scheiermann C. Circadian rhythms in leukocyte trafficking. Semin Immunopathol 2014;36:149-62.

33. Lasselin J, Rehman JU, Akerstedt T, Lekander M, Axelsson J. Effect of long-term sleep restriction and subsequent recovery sleep on the diurnal rhythms of white blood cell subpopulations. Brain Behav Immun 2015;47:93-9.

34. Mazzoccoli G, Correra M, Bianco G, De Cata A, Balzanelli M, Giuliani A, Tarquini R. Age-related changes of neuro-endocrine-immune interactions in healthy humans. J Biol Regul Homeost Agents 1997;11:143-7.

35. Mazzoccoli G, Carughi S, Sperandeo M, Pazienza V, Giuliani F, Greco A. Alteration of circadian rhythmicity of $c d 3+c d 4+$ lymphocyte subpopulation in healthy aging. J Biol Regul Homeost Agents 2011;25:405-16.

36. Fraser CG, Wilkinson SP, Neville RG, Knox JD, King JF, MacWalter RS. Biologic variation of common hematologic laboratory quantities in the elderly. Am J Clin Pathol 1989;92:465-70.

37. Iglesias $\mathrm{N}$, Petersen $\mathrm{PH}$, Ricos $\mathrm{C}$. Power function of the refence change value in relation to cut-off points, reference intervals and index of individuality. Clin Chem Lab Med 2005;43:4418 . 


\section{Supplementary figures and tables}

\begin{tabular}{|c|c|c|}
\hline Parameter & $\begin{array}{l}\text { Excluded subjects because of } \\
\text { incompleteness of sample collec- } \\
\text { tion or instable medical situation }\end{array}$ & $\begin{array}{l}\text { Cochran's C test } \\
\text { (analytical outliers) }\end{array}$ \\
\hline Neutrophils & Subjects $1,8,15,20$ and 23 & $\begin{array}{l}\text { Subject } 5 \text {, measurement } 5 \\
\text { Subject 12, measurement } 8 \\
\text { Subject 16, measurement } 1\end{array}$ \\
\hline Leukocytes & Subjects $1,8,15,20$ and 23 & $\begin{array}{l}\text { Subject } 12, \text { measurement } 8 \\
\text { Subject } 16, \text { measurement } 1\end{array}$ \\
\hline Lymphocytes & Subjects $1,8,15,20$ and 23 & None \\
\hline Eosinophils & Subjects 1, 8, 15, 20 and 23 & None \\
\hline Basophils & Subjects $1,8,15,20$ and 23 & None \\
\hline Monocytes & Subjects $1,8,15,20$ and 23 & Subject 12 , measurement 20 \\
\hline Hemoglobin & Subjects 1,8 and 20 & $\begin{array}{l}\text { Subject 2, measurement } 11 \\
\text { Subject 11, measurement } 1 \\
\text { Subject 23, measurement } 6\end{array}$ \\
\hline Hematocrit & Subjects 1,8 and 20 & $\begin{array}{l}\text { Subject } 10, \text { measurement } 12 \\
\text { Subject } 24, \text { measurement } 14\end{array}$ \\
\hline Erythrocytes & Subjects 1,8 and 20 & None \\
\hline Reticulocytes, count & Subjects 1, 8 and 20 & $\begin{array}{l}\text { Subject } 7, \text { measurement } 24 \\
\text { Subject } 11, \text { measurement } 24 \\
\text { Subject } 17, \text { measurement } 13\end{array}$ \\
\hline Reticulocytes, percentage & Subjects 1,8 and 20 & Subject 7, measurement 24 \\
\hline Platelets & Subjects 1,8 and 20 & $\begin{array}{l}\text { Subject 4, measurement } 4 \\
\text { Subject 9, measurement } 9 \\
\text { Subject 10, measurement } 8 \\
\text { Subject } 12 \text {, measurement } 8 \\
\text { Subject } 14, \text { measurement } 1 \\
\text { Subject } 15, \text { measurement } 3 \\
\text { Subject } 17, \text { measurement } 24 \\
\text { Subject } 24, \text { measurement } 15\end{array}$ \\
\hline Plateletcrit & Subjects 1,8 and 20 & Subject 13, measurement 7 \\
\hline Platelet Distribution Width (PDW) & Subjects $1,8,15,20$ and 23 & None \\
\hline Mean Platelet Volume (MPV) & Subjects $1,8,15,20$ and 23 & None \\
\hline
\end{tabular}

\begin{tabular}{|c|c|c|}
\hline Immature Platelet Fraction (IPF) & Subjects 1, 8, 15, 20 and 23 & Subject 9, measurement 9 \\
\hline Red Cell Distribution Width (RDW) & Subjects $1,8,15,20$ and 23 & None \\
\hline Mean Corpuscular Volume (MCV) & Subjects $1,8,15,20$ and 23 & $\begin{array}{l}\text { Subject } 11 \text {, measurement } 12 \\
\text { Subject } 21 \text {, measurement } 19\end{array}$ \\
\hline Mean Corpuscular Hemoglobin (MCH) & Subjects $1,8,15,20$ and 23 & Subject 7, measurement 20 \\
\hline $\begin{array}{l}\text { Mean Corpuscular Hemoglobin } \\
\text { Concentration (MCHC) }\end{array}$ & Subjects $1,8,15,20$ and 23 & $\begin{array}{l}\text { Subject } 7 \text {, measurement } 20 \\
\text { Subject } 16 \text {, measurement } 20\end{array}$ \\
\hline
\end{tabular}


Burnett test

(analytical outliers)

Cochran's C test

within-subject (non-homogeneity)

Subject 19

Subjects 14 and 19

None

Subject 10, measurement 24

Subject 12, measurement 1

Subject 24, measurement 16

\begin{tabular}{lll} 
None & Subject 12 & None \\
None & Subject 5 & None \\
\hline Subject 4, measurement 14 & None & None \\
Subject 24, measurement 16 & Subject 18 & None \\
None & Subject 19 & None
\end{tabular}

None

Subjects 6, 7, 16 and 19

None

None

Subject 19

None

None

None

None

None

Subject 12, measurement 16

Subject 2

None

Subject 18

None

\begin{tabular}{lll} 
None & Subjects 14, 16, 22 and 24 & None \\
Subject 14, measurement 17 & Subject 18 & None \\
$\begin{array}{l}\text { Subject 4, measurement 16 } \\
\text { Subject 11, measurement 19 } \\
\text { Subject 14, measurement 17 } \\
\text { Subject 19, measurement 2 }\end{array}$ & Subject 18 & None \\
$\begin{array}{l}\text { Subject 11, measurements 11, 19 and 22 } \\
\text { Subject 14, measurement 17 } \\
\text { Subject 18, measurement 19 } \\
\text { Subject 24, measurement 23 }\end{array}$ & & None \\
None & & \\
Subject 2, measurement 3 and 18 & Subject 22 & None \\
\hline None & Subjects 16, and 22 & None \\
None & None & None \\
\hline
\end{tabular}


Supplemental figure 3.1. Diurnal rhythms of cortisol versus lymphocytes

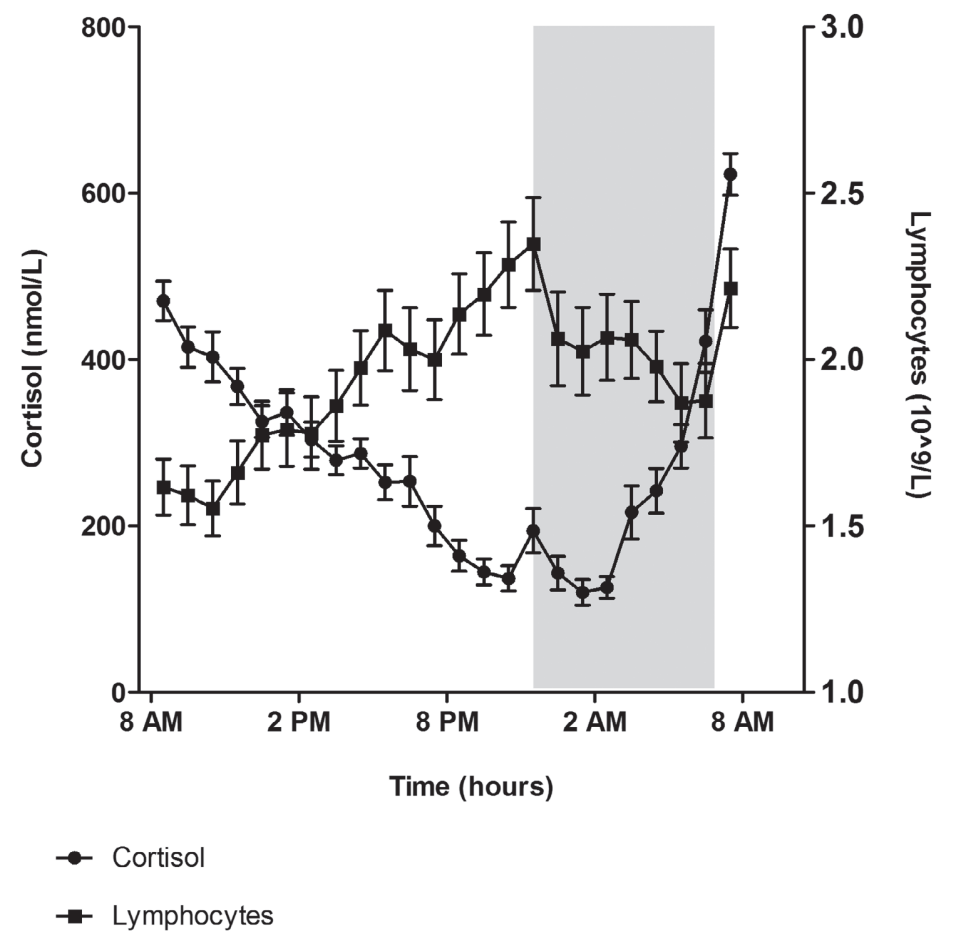

Values represent means \pm SEM $(n=18)$ of lymphocytes and cortisol within a day. Subjects slept between 11:30 p.m. and 7:00 a.m. (shaded area). 


\section{Supplemental table 3.2a: hour-to-hour RCVs of Leukocytes}

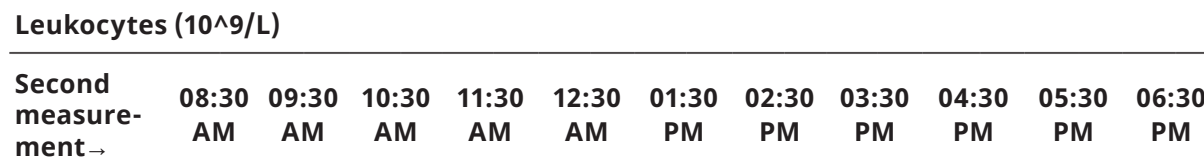

First mea-

surement $\downarrow$

\begin{tabular}{|c|c|c|c|c|c|c|c|c|c|c|}
\hline 08:30 AM & -1121 & -922 & -427 & -131 & -130 & -527 & -527 & -229 & -229 & -527 \\
\hline 09:30 AM & & -1517 & -1022 & -625 & -725 & -1021 & -1121 & -824 & -824 & -1021 \\
\hline 10:30 AM & & & -1120 & -824 & -823 & -1220 & -1220 & -922 & -922 & -1220 \\
\hline 11:30 AM & & & & -1319 & -1319 & -1615 & -1615 & -1418 & -1418 & -1615 \\
\hline 12:30 AM & & & & & -1615 & -1912 & -2012 & -1715 & -1714 & -1912 \\
\hline 01:30 PM & & & & & & -1913 & -1912 & -1715 & -1715 & -1913 \\
\hline 02:30 PM & & & & & & & -1616 & -1418 & -1418 & -1616 \\
\hline 03:30 PM & & & & & & & & -1318 & -1318 & -1616 \\
\hline 04:30 PM & & & & & & & & & -1616 & -1814 \\
\hline 05:30 PM & & & & & & & & & & -1814 \\
\hline \multicolumn{11}{|l|}{ 06:30 PM } \\
\hline \multicolumn{11}{|l|}{ 07:30 PM } \\
\hline \multicolumn{11}{|l|}{ 08:30 PM } \\
\hline \multicolumn{11}{|l|}{ 09:30 PM } \\
\hline \multicolumn{11}{|l|}{ 10:30 PM } \\
\hline \multicolumn{11}{|l|}{ 11:30 PM } \\
\hline \multicolumn{11}{|l|}{ 12:30 PM } \\
\hline \multicolumn{11}{|l|}{ 01:30 AM } \\
\hline \multicolumn{11}{|l|}{ 02:30 AM } \\
\hline \multicolumn{11}{|l|}{ 03:30 AM } \\
\hline \multicolumn{11}{|l|}{ 04:30 AM } \\
\hline \multicolumn{11}{|l|}{ 05:30 AM } \\
\hline \multicolumn{11}{|l|}{ 06:30 AM } \\
\hline 07:30 AM & & & & & & & & & & \\
\hline
\end{tabular}

The vertical column corresponds to the time of the first measurement and the horizontal row corresponds to the time of the second measurement. Each box indicates the percentage change range that is considered 'normal' $(95 \% \mathrm{CI})$, relative to the first measurement. If a percentage change between two consecutive measurements is outside this range, the change can be considered statistically significant. 


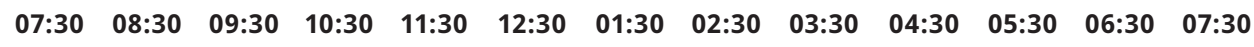 $\begin{array}{llllllllllll}\text { PM } & \text { PM } & \text { PM } & \text { PM } & \text { PM } & \text { PM } & \text { AM } & \text { AM } & \text { AM } & \text { AM } & \text { AM } & \text { AM }\end{array}$}

\begin{tabular}{|c|c|c|c|c|c|c|c|c|c|c|c|c|}
\hline-527 & -130 & -229 & -230 & 032 & -724 & -625 & -625 & -823 & -1022 & -1121 & -1120 & 133 \\
\hline-1121 & -725 & -824 & -724 & -626 & -1319 & -1220 & -1220 & -1418 & -1516 & -1616 & -1615 & -427 \\
\hline-1220 & -823 & -922 & -923 & -725 & -1418 & -1319 & -1319 & -1517 & -1715 & -1714 & -1814 & -626 \\
\hline-1615 & -1319 & -1418 & -1318 & -1220 & -1913 & -1814 & -1714 & $\begin{array}{ll}-19 & 12 \\
\end{array}$ & -2111 & -2110 & -2210 & -1121 \\
\hline-2012 & -1615 & -1714 & -1715 & -1517 & -2210 & -2111 & -2111 & -229 & -248 & -247 & -257 & -1418 \\
\hline-1912 & -1616 & -1715 & -1615 & -1517 & -2110 & -2011 & -2011 & -2210 & -248 & -248 & -257 & -1318 \\
\hline-1616 & -1319 & -1418 & -1319 & -1120 & -1813 & -1714 & -1715 & $\begin{array}{lll}-19 & 13 \\
\end{array}$ & -2111 & -2111 & -2210 & $\begin{array}{ll}-10 & 21 \\
\end{array}$ \\
\hline-1616 & -1219 & -1318 & -1319 & -1121 & $-18 \quad 14$ & -1715 & -1715 & -1913 & -2011 & -2111 & -2110 & $\begin{array}{ll}-10 & 22 \\
\end{array}$ \\
\hline-1813 & -1517 & -1616 & -1516 & -1418 & -2011 & -1912 & -1912 & $-21 \quad 10$ & -239 & -238 & -248 & -1319 \\
\hline-1813 & -1517 & -1616 & -1516 & -1418 & -2011 & -1912 & -1912 & -2110 & -239 & -238 & -248 & -1219 \\
\hline \multirow[t]{13}{*}{-1616} & -1319 & -1418 & -1319 & -1120 & -1813 & -1714 & -1715 & -1913 & -2111 & -2111 & -2210 & -1021 \\
\hline & -1219 & -1318 & -1319 & $\begin{array}{ll}-11 & 21 \\
\end{array}$ & -1814 & -1715 & -1715 & $\begin{array}{lll}-19 & 13\end{array}$ & -2011 & -2111 & -2110 & -1022 \\
\hline & & -1715 & -1615 & -1517 & -2110 & -2011 & -2011 & -2210 & -248 & -248 & -257 & -1318 \\
\hline & & & -1516 & -1418 & -2011 & $\begin{array}{lll}-19 & 12 \\
\end{array}$ & $\begin{array}{l}-1912 \\
\end{array}$ & -2111 & -239 & -238 & -248 & -1219 \\
\hline & & & & -1417 & -2111 & -2012 & -2012 & -2210 & -238 & -248 & -247 & -1319 \\
\hline & & & & & -229 & $-21 \quad 10$ & -2110 & -238 & -257 & -256 & -266 & -1517 \\
\hline & & & & & & -1517 & -1517 & -1715 & -1813 & -1913 & -1912 & -824 \\
\hline & & & & & & & -1616 & $-18 \quad 14$ & -1912 & -2012 & -2011 & -923 \\
\hline & & & & & & & & -1814 & -1912 & -2012 & -2011 & -923 \\
\hline & & & & & & & & & -1714 & $\begin{array}{lll}-18 & 14\end{array}$ & -1913 & -725 \\
\hline & & & & & & & & & & -1615 & -1715 & -527 \\
\hline & & & & & & & & & & & -1615 & -427 \\
\hline & & & & & & & & & & & & -428 \\
\hline
\end{tabular}




\section{Supplemental table 3.2b: hour-to-hour RCVs of Lymphocytes}

\section{Lymphocytes $\left(10^{\wedge} 9 / \mathrm{L}\right)$}

\begin{tabular}{|c|c|c|c|c|c|c|c|c|c|c|c|}
\hline $\begin{array}{l}\text { Second } \\
\text { measure- } \\
\text { ment } \rightarrow\end{array}$ & $\begin{array}{c}08: 30 \\
\text { AM }\end{array}$ & $\begin{array}{c}\text { 09:30 } \\
\text { AM }\end{array}$ & $\begin{array}{c}10: 30 \\
\text { AM }\end{array}$ & $\begin{array}{c}11: 30 \\
\text { AM }\end{array}$ & $\begin{array}{c}12: 30 \\
\text { AM }\end{array}$ & $\begin{array}{c}\text { 01:30 } \\
\text { PM }\end{array}$ & $\begin{array}{c}\text { 02:30 } \\
\text { PM }\end{array}$ & $\begin{array}{l}\text { 03:30 } \\
\text { PM }\end{array}$ & $\begin{array}{c}\text { 04:30 } \\
\text { PM }\end{array}$ & $\begin{array}{c}\text { 05:30 } \\
\text { PM }\end{array}$ & $\begin{array}{c}\text { 06:30 } \\
\text { PM }\end{array}$ \\
\hline \multicolumn{12}{|l|}{$\begin{array}{l}\text { First mea- } \\
\text { surement } \downarrow\end{array}$} \\
\hline 08:30 AM & & -2622 & -2920 & -2226 & -1633 & -1533 & -1732 & -1236 & -345 & 554 & 048 \\
\hline 09:30 AM & & & -2722 & -2028 & -1335 & -1335 & -1534 & -1038 & -147 & 856 & 251 \\
\hline 10:30 AM & & & & $\begin{array}{lll}-18 & 31\end{array}$ & -1038 & -1039 & -1237 & -741 & 251 & 1260 & 654 \\
\hline 11:30 AM & & & & & -1831 & -1731 & -1930 & -1434 & -543 & 352 & -246 \\
\hline 12:30 AM & & & & & & -2425 & -2523 & -2127 & -1336 & -544 & -1038 \\
\hline 01:30 PM & & & & & & & -2623 & -2227 & -1335 & -543 & -1138 \\
\hline 02:30 PM & & & & & & & & -2028 & -1237 & -445 & -939 \\
\hline 03:30 PM & & & & & & & & & -1632 & -840 & -1335 \\
\hline 04:30 PM & & & & & & & & & & -1731 & $-22 \quad 27$ \\
\hline 05:30 PM & & & & & & & & & & & -2920 \\
\hline \multicolumn{12}{|l|}{ 06:30 PM } \\
\hline \multicolumn{12}{|l|}{ 07:30 PM } \\
\hline \multicolumn{12}{|l|}{ 08:30 PM } \\
\hline \multicolumn{12}{|l|}{ 09:30 PM } \\
\hline \multicolumn{12}{|l|}{ 10:30 PM } \\
\hline \multicolumn{12}{|l|}{ 11:30 PM } \\
\hline \multicolumn{12}{|l|}{ 12:30 PM } \\
\hline \multicolumn{12}{|l|}{ 01:30 AM } \\
\hline \multicolumn{12}{|l|}{ 02:30 AM } \\
\hline \multicolumn{12}{|l|}{ 03:30 AM } \\
\hline \multicolumn{12}{|l|}{ 04:30 AM } \\
\hline \multicolumn{12}{|l|}{ 05:30 AM } \\
\hline \multicolumn{12}{|l|}{ 06:30 AM } \\
\hline 07:30 AM & & & & & & & & & & & \\
\hline
\end{tabular}

The vertical column corresponds to the time of the first measurement and the horizontal row corresponds to the time of the second measurement. Each box indicates the percentage change range that is considered 'normal' (95\% CI), relative to the first measurement. If a percentage change between two consecutive measurements is outside this range, the change can be considered statistically significant. 


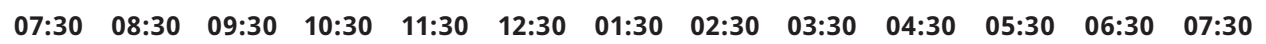

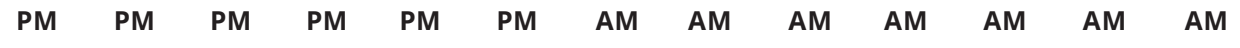

\begin{tabular}{|c|c|c|c|c|c|c|c|c|c|c|c|c|}
\hline-345 & 655 & 957 & 1463 & 1967 & 250 & -147 & 251 & 251 & -346 & -840 & -840 & 1261 \\
\hline-147 & 957 & 1160 & 1766 & 2270 & 553 & 150 & 553 & 553 & 048 & -642 & -642 & 1563 \\
\hline 251 & 1361 & 1564 & 2170 & 2674 & 857 & 553 & 857 & 857 & 352 & -346 & -346 & 1967 \\
\hline-543 & 452 & 655 & 1260 & 1665 & 048 & -345 & 048 & 048 & -544 & -1038 & -1038 & 1058 \\
\hline-1336 & -444 & -247 & 452 & 856 & -841 & -1138 & -841 & -841 & -1236 & -1731 & -1831 & 150 \\
\hline-1335 & -544 & -246 & 351 & 755 & -840 & -1137 & -840 & -840 & -1336 & -1830 & -1830 & 149 \\
\hline-1237 & -346 & 048 & 553 & 957 & -742 & -1039 & -742 & -742 & -1137 & -1632 & -1632 & 351 \\
\hline-1632 & -741 & -543 & 048 & 452 & -1137 & -1434 & -1137 & -1137 & -1533 & -2028 & -2128 & -246 \\
\hline-2424 & -1632 & -1434 & -939 & -643 & -2029 & -2226 & -2029 & -2029 & -2425 & -2820 & -2820 & -1137 \\
\hline-3117 & -2325 & $-22 \quad 27$ & -1731 & -1434 & -2722 & -2919 & -2722 & -2722 & -3018 & -3514 & -3513 & -1929 \\
\hline \multirow[t]{13}{*}{-2722} & -1930 & -1732 & -1236 & -940 & $-22 \quad 26$ & -2524 & -2226 & $-22 \quad 26$ & -2622 & -3118 & -3118 & -1434 \\
\hline & -1632 & -1434 & -939 & -643 & -2029 & -2226 & -2029 & -2029 & -2425 & -2820 & -2820 & -1137 \\
\hline & & $-22 \quad 26$ & -1830 & -1534 & -2721 & -3018 & -2721 & -2721 & -3117 & -3513 & -3613 & -2029 \\
\hline & & & -2028 & -1732 & -2919 & -3217 & -2919 & -2919 & -3316 & -3711 & -3711 & -2227 \\
\hline & & & & $\begin{array}{ll}-21 & 27\end{array}$ & -3315 & -3513 & -3315 & -3315 & -3612 & -418 & -418 & -2623 \\
\hline & & & & & -3613 & -3810 & -3613 & -3613 & -399 & -435 & -435 & -2920 \\
\hline & & & & & & -2722 & -2424 & -2424 & -2820 & -3216 & -3316 & -1632 \\
\hline & & & & & & & $\begin{array}{ll}-21 & 27\end{array}$ & $-22 \quad 27$ & -2523 & -3018 & -3018 & -1335 \\
\hline & & & & & & & & -2424 & -2820 & -3316 & -3316 & -1632 \\
\hline & & & & & & & & & -2820 & -3216 & -3316 & -1632 \\
\hline & & & & & & & & & & -2919 & -2919 & -1236 \\
\hline & & & & & & & & & & & -2424 & -742 \\
\hline & & & & & & & & & & & & -642 \\
\hline
\end{tabular}




\section{Supplemental table 3.2c: hour-to-hour RCVs of Eosinophils}

\section{Eosinophils (10^9/L)}

\begin{tabular}{|c|c|c|c|c|c|c|c|c|c|c|c|}
\hline $\begin{array}{l}\text { Second } \\
\text { measure- } \\
\text { ment } \rightarrow\end{array}$ & $\begin{array}{c}08: 30 \\
\text { AM }\end{array}$ & $\begin{array}{c}09: 30 \\
\text { AM }\end{array}$ & $\begin{array}{c}10: 30 \\
\text { AM }\end{array}$ & $\begin{array}{c}11: 30 \\
\text { AM }\end{array}$ & $\begin{array}{c}12: 30 \\
A M\end{array}$ & $\begin{array}{c}\text { 01:30 } \\
\text { PM }\end{array}$ & $\begin{array}{c}\text { 02:30 } \\
\text { PM }\end{array}$ & $\begin{array}{c}\text { 03:30 } \\
\text { PM }\end{array}$ & $\begin{array}{c}\text { 04:30 } \\
\text { PM }\end{array}$ & $\begin{array}{c}\text { 05:30 } \\
\text { PM }\end{array}$ & $\begin{array}{c}\text { 06:30 } \\
\text { PM }\end{array}$ \\
\hline \multicolumn{12}{|l|}{$\begin{array}{l}\text { First mea- } \\
\text { surement } \downarrow\end{array}$} \\
\hline 08:30 AM & & -6528 & -7518 & -7419 & -7320 & -7320 & -6726 & -6231 & -5340 & -5340 & -5241 \\
\hline 09:30 AM & & & -5835 & -5835 & -5637 & -5637 & -4944 & -4449 & -3360 & -3261 & -3162 \\
\hline 10:30 AM & & & & -4647 & -4449 & -4449 & -3657 & -3063 & -1776 & -1776 & -1578 \\
\hline 11:30 AM & & & & & -4449 & -4449 & -3657 & -3063 & -1776 & -1776 & -1677 \\
\hline 12:30 AM & & & & & & -4746 & -3954 & -3261 & -2073 & -2073 & -1974 \\
\hline 01:30 PM & & & & & & & -3855 & -3261 & -2073 & -2073 & -1875 \\
\hline 02:30 PM & & & & & & & & -4152 & -3063 & -2964 & -2865 \\
\hline 03:30 PM & & & & & & & & & -3657 & -3657 & -3459 \\
\hline 04:30 PM & & & & & & & & & & -4647 & -4548 \\
\hline 05:30 PM & & & & & & & & & & & -4548 \\
\hline \multicolumn{12}{|l|}{ 06:30 PM } \\
\hline \multicolumn{12}{|l|}{ 07:30 PM } \\
\hline \multicolumn{12}{|l|}{ 08:30 PM } \\
\hline \multicolumn{12}{|l|}{ 09:30 PM } \\
\hline \multicolumn{12}{|l|}{ 10:30 PM } \\
\hline \multicolumn{12}{|l|}{ 11:30 PM } \\
\hline \multicolumn{12}{|l|}{ 12:30 PM } \\
\hline \multicolumn{12}{|l|}{ 01:30 AM } \\
\hline \multicolumn{12}{|l|}{ 02:30 AM } \\
\hline \multicolumn{12}{|l|}{ 03:30 AM } \\
\hline \multicolumn{12}{|l|}{ 04:30 AM } \\
\hline \multicolumn{12}{|l|}{ 05:30 AM } \\
\hline \multicolumn{12}{|l|}{ 06:30 AM } \\
\hline 07:30 AM & & & & & & & & & & & \\
\hline
\end{tabular}

The vertical column corresponds to the time of the first measurement and the horizontal row corresponds to the time of the second measurement. Each box indicates the percentage change range that is considered 'normal' (95\% CI), relative to the first measurement. If a percentage change between two consecutive measurements is outside this range, the change can be considered statistically significant. 


\begin{tabular}{|c|c|c|c|c|c|c|c|c|c|c|c|c|}
\hline 07:30 & 08:30 & 09:30 & $10: 30$ & $11: 30$ & $12: 30$ & 01:30 & 02:30 & 03:30 & 04:30 & 05:30 & 06:30 & 07:30 \\
\hline PM & PM & PM & PM & PM & PM & $\mathrm{AM}$ & AM & $\mathrm{AM}$ & AM & AM & AM & AM \\
\hline
\end{tabular}

\begin{tabular}{|c|c|c|c|c|c|c|c|c|c|c|c|c|}
\hline-4449 & -4152 & -4350 & -4449 & -3756 & -3855 & -3459 & -3558 & -3855 & -3360 & -3360 & -3459 & -3261 \\
\hline-2272 & -1776 & -1974 & -2172 & -1281 & -1479 & -984 & -1182 & -1479 & -786 & -786 & -984 & -687 \\
\hline-489 & 093 & -291 & -489 & 699 & 497 & 9102 & 8101 & 497 & 12105 & 12105 & 9102 & 13106 \\
\hline-588 & 093 & -291 & -489 & 699 & 397 & 9102 & 8101 & 497 & 11104 & + 11104 & 9102 & 13106 \\
\hline-885 & -390 & -687 & -885 & 396 & 093 & 598 & 497 & 194 & 8101 & 8101 & 699 & 9102 \\
\hline-885 & -390 & -588 & -786 & 396 & 093 & 699 & 598 & 194 & 8101 & 8101 & 699 & 9102 \\
\hline-1875 & -1479 & -1677 & -1875 & -885 & -1182 & -687 & -786 & -1083 & -390 & -390 & -687 & -291 \\
\hline-2568 & -2172 & -2370 & -2568 & -1677 & -1875 & -1380 & -1479 & -1875 & -1182 & -1182 & -1380 & -1083 \\
\hline-3756 & -3360 & -3558 & -3657 & -2865 & -3063 & -2667 & -2766 & -3063 & -2469 & -2469 & -2667 & -2370 \\
\hline-3756 & -3360 & -3558 & -3756 & -2865 & -3063 & -2667 & -2766 & -3063 & -2469 & -2469 & -2667 & -2370 \\
\hline \multirow[t]{13}{*}{-3855} & -3558 & -3657 & -3855 & -3063 & -3261 & -2865 & -2964 & -3261 & -2667 & -2667 & -2865 & -2568 \\
\hline & -4350 & -4548 & -4647 & -3954 & -4152 & -3756 & -3855 & -4053 & -3558 & -3558 & -3756 & -3459 \\
\hline & & -4845 & -4944 & -4251 & -4449 & -4053 & -4152 & -4449 & -3954 & -3954 & -4053 & -3855 \\
\hline & & & -4845 & -4152 & -4251 & -3954 & -3954 & -4251 & -3756 & -3756 & -3955 & -3657 \\
\hline & & & & -3954 & -4152 & -3756 & -3855 & -4152 & -3558 & -3558 & -3756 & -3558 \\
\hline & & & & & -4845 & -4548 & -4548 & -4845 & -4350 & -4350 & -4548 & -4251 \\
\hline & & & & & & -4350 & -4449 & -4647 & -4152 & -4152 & -4350 & -4053 \\
\hline & & & & & & & -4746 & -5043 & -4548 & -4548 & -4647 & -4449 \\
\hline & & & & & & & & -4944 & -4449 & -4449 & -4647 & -4350 \\
\hline & & & & & & & & & -4251 & -4251 & -4350 & -4152 \\
\hline & & & & & & & & & & -4747 & -4845 & -4647 \\
\hline & & & & & & & & & & & -4845 & -4647 \\
\hline & & & & & & & & & & & & -4449 \\
\hline
\end{tabular}




\section{Supplemental table 3.2d: hour-to-hour RCVs of Basophils}

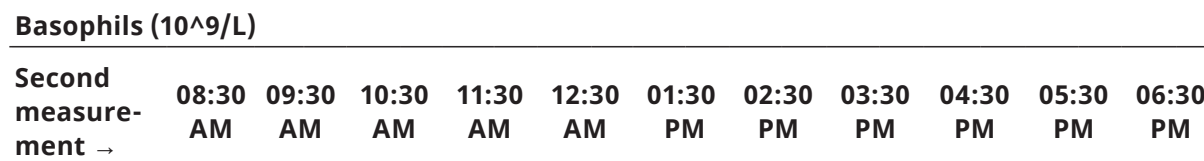

First mea-

surement $\downarrow$

\begin{tabular}{|c|c|c|c|c|c|c|c|c|c|c|}
\hline 08:30 AM & -7967 & -8066 & -7670 & -7570 & -7274 & -7670 & -7175 & -7274 & -6680 & -7175 \\
\hline 09:30 AM & & -7571 & -7075 & -6976 & -6680 & -7075 & -6581 & -6680 & -5987 & -6581 \\
\hline 10:30 AM & & & -6877 & -6878 & -6482 & -6877 & -6383 & -6482 & -5789 & -6383 \\
\hline 11:30 AM & & & & -7274 & -6878 & -7373 & -6878 & -6977 & -6284 & -6878 \\
\hline 12:30 AM & & & & & -6977 & -7472 & -6877 & -7076 & -6383 & -6977 \\
\hline 01:30 PM & & & & & & -7768 & -7274 & -7372 & -6779 & -7273 \\
\hline 02:30 PM & & & & & & & -6878 & -6977 & -6284 & -6878 \\
\hline 03:30 PM & & & & & & & & -7472 & -6878 & -7373 \\
\hline 04:30 PM & & & & & & & & & -6679 & -7274 \\
\hline 05:30 PM & & & & & & & & & & -7868 \\
\hline \multicolumn{11}{|l|}{ 06:30 PM } \\
\hline \multicolumn{11}{|l|}{ 07:30 PM } \\
\hline \multicolumn{11}{|l|}{ 08:30 PM } \\
\hline \multicolumn{11}{|l|}{ 09:30 PM } \\
\hline \multicolumn{11}{|l|}{ 10:30 PM } \\
\hline \multicolumn{11}{|l|}{ 11:30 PM } \\
\hline \multicolumn{11}{|l|}{ 12:30 PM } \\
\hline \multicolumn{11}{|l|}{ 01:30 AM } \\
\hline \multicolumn{11}{|l|}{ 02:30 AM } \\
\hline \multicolumn{11}{|l|}{ 03:30 AM } \\
\hline \multicolumn{11}{|l|}{ 04:30 AM } \\
\hline \multicolumn{11}{|l|}{ 05:30 AM } \\
\hline \multicolumn{11}{|l|}{ 06:30 AM } \\
\hline 07:30 AM & & & & & & & & & & \\
\hline
\end{tabular}

The vertical column corresponds to the time of the first measurement and the horizontal row corresponds to the time of the second measurement. Each box indicates the percentage change range that is considered 'normal' $(95 \% \mathrm{CI})$, relative to the first measurement. If a percentage change between two consecutive measurements is outside this range, the change can be considered statistically significant. 


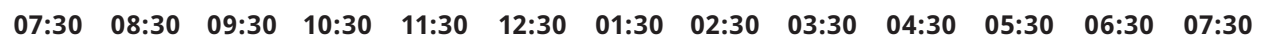

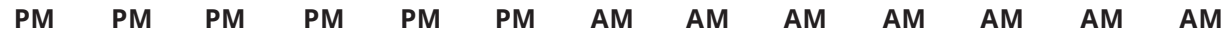

\begin{tabular}{|c|c|c|c|c|c|c|c|c|c|c|c|c|}
\hline-7075 & -7076 & -6977 & -6284 & -6977 & -8264 & -7967 & -7966 & -7670 & -8165 & -6679 & -7670 & -6185 \\
\hline-6482 & -6382 & -6383 & -5591 & -6383 & -7669 & -7373 & -7472 & -7075 & -7570 & -6086 & -7075 & -5492 \\
\hline-6283 & -6184 & -6185 & -5393 & -6185 & -7571 & -7175 & -7274 & -6877 & -7472 & -5888 & -6877 & -5294 \\
\hline-6779 & -6680 & -6680 & -5888 & -6580 & -7967 & -7570 & -7670 & -7373 & -7868 & -6383 & -7373 & -5789 \\
\hline-6878 & -6779 & -6679 & -5987 & -6680 & -8066 & -7670 & -7769 & -7472 & -7967 & -6482 & -7472 & -5888 \\
\hline-7274 & -7175 & -7075 & -6383 & -7076 & -8363 & -8066 & -8165 & -7768 & -8264 & -6878 & -7768 & -6284 \\
\hline-6779 & -6680 & -6680 & -5888 & -6580 & -7967 & -7570 & -7670 & -7373 & -7868 & -6383 & -7373 & -5789 \\
\hline-7273 & -7274 & -7175 & -6482 & -7175 & -8462 & -8065 & -8165 & -7868 & -8363 & -6877 & -7868 & -6383 \\
\hline-7174 & -7075 & -7076 & -6283 & -7076 & -8363 & -7966 & -8066 & -7769 & -8264 & -6779 & -7769 & -6284 \\
\hline-7768 & -7769 & -7670 & -6977 & -7670 & -8858 & -8561 & -8660 & -8363 & -8758 & -7472 & -8363 & -6877 \\
\hline \multirow[t]{13}{*}{-7274} & -7174 & -7175 & -6382 & -7075 & -8362 & -8066 & -8165 & -7868 & -8263 & -6878 & -7868 & -6283 \\
\hline & -7274 & -7274 & -6482 & -7174 & -8462 & -8165 & -8264 & -7867 & -8363 & -6977 & -7867 & -6382 \\
\hline & & -7273 & -6581 & -7274 & -8561 & -8264 & -8263 & -7967 & -8462 & -7076 & -7967 & -6482 \\
\hline & & & -6680 & -7373 & -8561 & -8264 & -8363 & -8066 & -8461 & -7075 & -8066 & -6581 \\
\hline & & & & -7966 & -9155 & -8858 & -8957 & -8660 & -9055 & -7769 & -8660 & -7274 \\
\hline & & & & & -8560 & -8264 & -8363 & -8066 & -8561 & -7175 & -8066 & -6581 \\
\hline & & & & & & -6976 & -7076 & -6779 & -7274 & -5690 & -6779 & -5096 \\
\hline & & & & & & & -7472 & -7075 & -7570 & -6086 & -7075 & -5492 \\
\hline & & & & & & & & -6976 & -7571 & -5987 & -6976 & -5393 \\
\hline & & & & & & & & & -7868 & -6383 & -7373 & -5789 \\
\hline & & & & & & & & & & -5789 & -6878 & -5195 \\
\hline & & & & & & & & & & & -8264 & -6878 \\
\hline & & & & & & & & & & & & -5789 \\
\hline
\end{tabular}




\section{Supplemental table 3.2e: hour-to-hour RCVs of Monocytes}

\begin{tabular}{|c|c|c|c|c|c|c|c|c|c|c|c|}
\hline Monocyte & $\left(10^{\wedge} 9 / \mathrm{L}\right.$ & & & & & & & & & & \\
\hline $\begin{array}{l}\text { Second } \\
\text { measure- } \\
\text { ment } \rightarrow\end{array}$ & $\begin{array}{c}08: 30 \\
\text { AM }\end{array}$ & $\begin{array}{c}\text { 09:30 } \\
\text { AM }\end{array}$ & $\begin{array}{c}10: 30 \\
\text { AM }\end{array}$ & $\begin{array}{c}11: 30 \\
\text { AM }\end{array}$ & $\begin{array}{c}12: 30 \\
\text { AM }\end{array}$ & $\begin{array}{c}\text { 01:30 } \\
\text { PM }\end{array}$ & $\begin{array}{c}\text { 02:30 } \\
\text { PM }\end{array}$ & $\begin{array}{c}\text { 03:30 } \\
\text { PM }\end{array}$ & $\begin{array}{c}\text { 04:30 } \\
\text { PM }\end{array}$ & $\begin{array}{c}\text { 05:30 } \\
\text { PM }\end{array}$ & $\begin{array}{c}06: 30 \\
\text { PM }\end{array}$ \\
\hline
\end{tabular}

\section{First mea-}

surement $\downarrow$

\begin{tabular}{|c|c|c|c|c|c|c|c|c|c|}
\hline 08:30 AM & $\begin{array}{llll}-40 & 26 & -48 & 17\end{array}$ & -3332 & -1847 & -3036 & -3728 & -3234 & -2442 & -1946 & -3431 \\
\hline 09:30 AM & -4224 & -2540 & -956 & -2244 & -3035 & -2442 & -1550 & -1155 & -2739 \\
\hline 10:30 AM & & -1551 & 368 & -1055 & -2046 & -1353 & -362 & 267 & -1649 \\
\hline 11:30 AM & & & -1848 & -2936 & -3728 & -3134 & -2342 & -1947 & -3431 \\
\hline 12:30 AM & & & & -4323 & -4916 & -4421 & -3728 & -3432 & -4719 \\
\hline 01:30 PM & & & & & -4025 & -3531 & -2739 & -2343 & -3728 \\
\hline 02:30 PM & & & & & & -2739 & -1847 & -1452 & -3036 \\
\hline 03:30 PM & & & & & & & -2541 & -2145 & -3630 \\
\hline 04:30 PM & & & & & & & & -2937 & -4323 \\
\hline 05:30 PM & & & & & & & & & -4619 \\
\hline \multicolumn{10}{|l|}{ 06:30 PM } \\
\hline \multicolumn{10}{|l|}{ 07:30 PM } \\
\hline \multicolumn{10}{|l|}{ 08:30 PM } \\
\hline \multicolumn{10}{|l|}{ 09:30 PM } \\
\hline \multicolumn{10}{|l|}{ 10:30 PM } \\
\hline \multicolumn{10}{|l|}{ 11:30 PM } \\
\hline \multicolumn{10}{|l|}{ 12:30 PM } \\
\hline \multicolumn{10}{|l|}{ 01:30 AM } \\
\hline \multicolumn{10}{|l|}{ 02:30 AM } \\
\hline \multicolumn{10}{|l|}{ 03:30 AM } \\
\hline \multicolumn{10}{|l|}{ 04:30 AM } \\
\hline \multicolumn{10}{|l|}{ 05:30 AM } \\
\hline \multicolumn{10}{|l|}{ 06:30 AM } \\
\hline 07:30 AM & & & & & & & & & \\
\hline
\end{tabular}

The vertical column corresponds to the time of the first measurement and the horizontal row corresponds to the time of the second measurement. Each box indicates the percentage change range that is considered 'normal' (95\% CI), relative to the first measurement. If a percentage change between two consecutive measurements is outside this range, the change can be considered statistically significant. 


$\begin{array}{ccccccccccccc}07: 30 & 08: 30 & 09: 30 & 10: 30 & 11: 30 & 12: 30 & 01: 30 & 02: 30 & 03: 30 & 04: 30 & 05: 30 & 06: 30 & 07: 30 \\ \text { PM } & \text { PM } & \text { PM } & \text { PM } & \text { PM } & \text { PM } & \text { AM } & \text { AM } & \text { AM } & \text { AM } & \text { AM } & \text { AM } & \text { AM }\end{array}$

\begin{tabular}{|c|c|c|c|c|c|c|c|c|c|c|c|}
\hline $\begin{array}{lll}-30 & 36 & -19 \\
\end{array}$ & -2046 & -1848 & -1650 & -2540 & -2541 & -2936 & -2837 & -2937 & -2738 & -2639 & -2144 \\
\hline$-2244-1056$ & -1154 & -956 & -759 & -1748 & -1749 & -2144 & -2045 & -2145 & -1947 & -1848 & -1353 \\
\hline$-1055 \quad 368$ & 167 & 369 & 671 & -560 & -561 & -1056 & -957 & -956 & -758 & -659 & 065 \\
\hline $\begin{array}{llll}-29 & 36 & -18 & 47\end{array}$ & -1946 & -1848 & -1550 & -2541 & -2541 & -2937 & -2838 & -2837 & -2739 & -2640 & -2145 \\
\hline$-4323-3332$ & -3431 & -3233 & -3135 & -3927 & -3927 & -4223 & -4124 & -4224 & -4025 & -4026 & -3530 \\
\hline 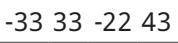 & -2342 & -2244 & -1946 & -2937 & -2837 & -3233 & -3134 & -3234 & -3035 & -2936 & -2541 \\
\hline $\begin{array}{lll}-25 & 41 & -13 \\
52\end{array}$ & -1451 & -1253 & -1055 & -2045 & -2046 & -2441 & -2343 & -2442 & -2244 & -2145 & -1650 \\
\hline $\begin{array}{lll}-31 & 35 & -20 \\
\end{array}$ & -2145 & -1946 & -1748 & -2739 & -2639 & -3035 & -2936 & -3036 & -2837 & -2738 & -2243 \\
\hline $\begin{array}{llll}-38 & 27 & -28 & 37\end{array}$ & -2936 & -2838 & -2640 & -3431 & -3432 & -3828 & -3729 & -3728 & -3630 & -3530 & -3135 \\
\hline$-4224-3233$ & -3332 & -3234 & -3036 & -3827 & -3828 & -4124 & -4125 & -4124 & -4026 & -3927 & -3431 \\
\hline \multirow[t]{13}{*}{$\begin{array}{lll}-28 & 38 & -17 \\
49\end{array}$} & -1848 & -1650 & -1452 & -2342 & -2342 & -2738 & -2639 & -2739 & -2540 & -2441 & -1946 \\
\hline & -2342 & -2144 & -1946 & -2937 & -2837 & -3233 & -3134 & -3234 & -3035 & -2936 & -2541 \\
\hline & -3432 & -3233 & -3035 & -3927 & -3827 & -4223 & -4124 & -4224 & -4025 & -3926 & -3531 \\
\hline & & -3134 & -2936 & -3828 & -3728 & -4124 & -4025 & -4125 & -3926 & $\begin{array}{ll}-38 & 27\end{array}$ & -3432 \\
\hline & & & -3135 & -3926 & -3927 & -4323 & -4224 & -4223 & -4125 & -4026 & -3630 \\
\hline & & & & -4125 & -4125 & -4421 & -4322 & -4422 & -4223 & -4224 & -3728 \\
\hline & & & & & -3233 & -3629 & -3530 & -3630 & -3431 & -3432 & -2937 \\
\hline & & & & & & -3729 & -3630 & -3629 & -3531 & -3432 & -2936 \\
\hline & & & & & & & -3234 & -3233 & -3135 & -3036 & -2541 \\
\hline & & & & & & & & -3332 & -3234 & -3135 & -2639 \\
\hline & & & & & & & & & -3134 & -3035 & -2540 \\
\hline & & & & & & & & & & -3234 & -2738 \\
\hline & & & & & & & & & & & -2837 \\
\hline
\end{tabular}




\section{Chapter 4}




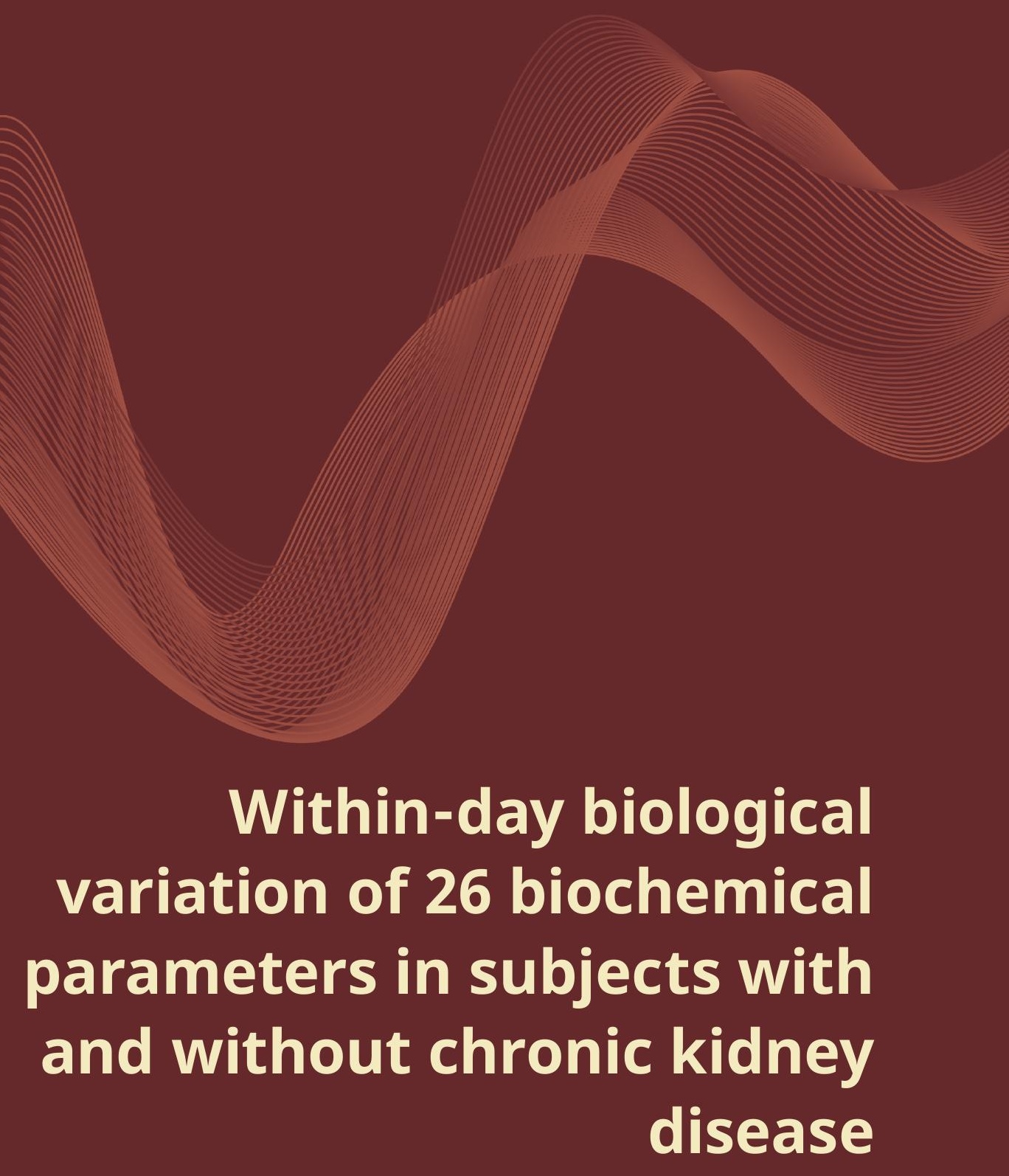

J.M. Hilderink, N. van der Linden, D.M. Kimenai, L.J.J. Klinkenberg, O. Bekers, R.P. Koopmans, S.J.R. Meex 


\section{Abstract}

\section{Background}

Within-day biological variation data is important to be able to make reliable estimates whether changes between two serial laboratory results within the day, are 'real changes' or not. Biological variation is believed to be of the same magnitude in health and disease, if the illness is chronic and the disease is stable. However, it is unclear if this holds true for within-day biological variation and for parameters that have not been studied yet.

This study was performed to present an overview of within-day biological variation data of 26 biochemical parameters, some routine and some research parameters. The assumption was tested if within-day biological variation is similar for people with and without a chronic kidney disease (CKD).

\section{Methods}

During 24h, hourly blood samples were collected from 24 subjects without CKD and 20 subjects with CKD. Components of variation, as well as Reference Change Values (RCV's) were calculated for both study groups. Outlier and homogeneity analyses were performed before using a nested ANOVA test to determine components of biological variation, including within-subject biological variation $\left(\mathrm{CV}_{\mathrm{I}}\right)$, between-subject biological variation $\left(\mathrm{CV}_{\mathrm{G}}\right)$, analytical variation $\left(\mathrm{CV}_{\mathrm{A}}\right)$ and index of individuality (II).

\section{Results}

$\mathrm{CV}_{\mathrm{G}}$ was larger than $\mathrm{CV}_{\mathrm{I}}$ for all parameters in both study groups, except for sodium and phosphate. $\mathrm{CV}_{\mathrm{I}}$ was similar for the two study groups in 20 parameters (77\%). In the other parameters, $\mathrm{CV}_{\mathrm{I}}$ values differed. In both study groups, 24 out of 26 parameters (92\%) had an index of individuality $<0.6$.

In general, RCVs were of similar magnitudes for subjects with- and without CKD. The highest RCVs were found for triglycerides ( $59.1 \%$ and $48.8 \%$, respectively). The lowest RCVs were found for sodium ( $2.6 \%$ and $3.1 \%$, respectively).

\section{Conclusion}

Our findings imply that the within-day $\mathrm{CV}_{\mathrm{I}}$ is not affected by the presence of chronic (kidney) disease for most biochemical parameters. Observed RCV's were of similar magnitude for subjects with- and without CKD. Thus, the use of RCV's derived from healthy subjects for monitoring patients seems to be reasonable. 


\section{Background}

Biological variation studies have been widely performed in laboratory medicine. The past two decades, there has been an ever-increasing interest in the dissemination of guidelines from biological variation working groups (1-3). This initiative was designed to strive for more uniformity in the implementation of biological variation studies and to deliver 'high quality biological variation data' (4).

For most of the parameters, biological variation has already been studied and reported before. However, these studies are mostly about between-day biological variation $(5,6)$ Few papers report the within-day biological variation. Moreover, to the best of our knowledge, for some parameters, such as soluble transferrin receptor (sTfR) and homocystein (within-day) biological variation was never studied before.

Within-day biological variation data is important to be able to make reliable estimates whether changes between two serial laboratory results within the day, are 'real changes' or not. Besides, if a parameter has an intrinsic diurnal rhythm, within-day biological variation data can contribute if this parameter is measured on two different time points.

Also, with reliable biological variation data, reference change values (RCV) can be calculated to assess the significance of a change between two serial laboratory results from an individual. Moreover, availability of reliable (within-day) biological variation data is important for the definition of analytical performance specifications (7). Furthermore, the utility of population based reference values can be assessed by the calculation of the index of individuality (II), which is the ratio of the between-subject biological variation $\left(\mathrm{CV}_{\mathrm{G}}\right)$ and the within-subject biological variation $\left(\mathrm{CV}_{\mathrm{I}}\right)(8)$.

Biological variation is believed to be of the same magnitude in health and disease, if the illness is chronic and the disease is stable $(9,10)$. However, it is unclear if this holds true for within-day biological variation and for parameters that have not been studied yet.

This study was performed to present an overview of within-day biological variation data of 26 biochemical parameters, some routine and some research parameters. Besides, we assessed if within-day biological variation is similar for people with and without a chronic illness. 


\section{Materials and methods}

This study was approved by the Institutional Review Board and the ethics committee at Maastricht University, the Netherlands, and agrees with the World Medical Association Declaration of Helsinki. The study was performed in the period 2013-2015 at Maastricht University Medical Center. The registration numbers at www.clinicaltrials.org were: NCT02091427 and NCT02210897. All subjects provided written informed consent.

\section{Sample collection and handling}

This study included 44 subjects, 33 men and 11 women, aged between 39 and 83 years. Baseline characteristics were shown in table 1 . The subjects were divided into two study groups. The first study group consisted of 24 subjects without clinically diagnosed CKD (19 males and 5 females). The other study group consisted of 20 patients with CKD stage 3 or higher (eGFR $\leq 59 \mathrm{~mL} / \mathrm{min} / 1.73 \mathrm{~m}^{2}$ ) (11) (14 males and 6 females). This number of subjects per group provides adequate power to make reliable estimations about biological variation (power $=1.00, a=0.05)(12)$. The subjects are described in more detail in previous studies of our group (13-16).

Subjects were invited for a test day in sets of two or three persons per test day. On a test day, subjects arrived at the laboratory at 8:00 AM after an overnight fast. During the test day, subjects were restricted to the laboratory environment and were refrained from strenuous exercise. Mealtimes were standardized at 08:30 AM (breakfast), 12:30 AM (lunch) and 6:00 PM (dinner). Subjects went to bed at 11:30 PM and got up the next morning at 07:00 AM. Venous blood samples were drawn every hour, during 24 hour in ethylenediaminetraacetic acid (EDTA)-containing tubes using an intravenous cannula. During the night, an extending line was attached to the cannula to prevent sleep disturbance during blood sampling.

The first (baseline) blood sample was taken at 8:30 AM, and from then every hour during 24h (9:30 AM, 10:30 AM, etc.). Most of the blood samples were drawn by the same phlebotomist.

After each sample collection, serum/plasma was separated by centrifugation at $2700 \mathrm{~g}$ for 12 minutes at room temperature. Samples were then aliquoted and stored at $-80^{\circ} \mathrm{C}$ until analysis. 


\section{Analytical methods}

Measurements of all parameters were performed on a Cobas 8000 (Roche Diagnostics, Basel, Switzerland). All samples from half of the study population were analyzed in duplicate within a single run in order to estimate ${C V_{A}}_{A}$.

\section{Data analysis}

Data were CV-transformed in order to perform the CV-ANOVA (17). This nonparametric calculation has been shown to be robust and distribution-independent, which makes it suitable for estimating components of biological variation (17). Briefly, the CV-ANOVA method is based

Table 4.1. Baseline characteristics of the study participants $(n=43)$

\begin{tabular}{lrr} 
& Subjects without CKD $(\boldsymbol{n}=\mathbf{2 3})$ & Subjects with CKD $(\boldsymbol{n}=\mathbf{2 0})$ \\
\hline Age, years & $72 \pm 7$ & $66 \pm 12$ \\
Male gender & $19(83)$ & $14(70)$ \\
Body Mass Index & $26 \pm 4.5$ & $28 \pm 4$ \\
Systolic blood pressure, mmHg & $138.9 \pm 13.8$ & $136 \pm 19$ \\
Diastolic blood pressure & $67.4 \pm 7.5$ & $86 \pm 14$ \\
eGFR, $\mathrm{ml} / \mathrm{min} / 1.73 \mathrm{~m}^{2 a}$ & $76 \pm 19$ & $19.0 \pm 7.0$ \\
\hline
\end{tabular}

a for the estimation of GFR the CKD-EPI creatinine - cystatin C formula was used (21).

on the CV transformation with normalization of the data by dividing by each subject's mean value, and then performing the ANOVA. As recommended for biological variation studies, data were checked for homogeneity and outliers (18). Replicates were excluded until homoscedasticity was achieved on analytical and within-subject level (3). To verify the normality of the residuals, the Shapiro Wilk test was used (19).

Since some parameters are posture dependent and show a concentration drop during nighttime (such as albumin, total protein, transferrin, see figures 1 and 2), we calculated biological variation by using the first 15 consecutive measurements (08:30 AM - 11:30 PM), to make sure there was a steady state for each parameter. Besides, biological variation data were determined identically for all parameters since the number of time points was the same. 
The Dixon-Reed criterion was used to detect any outliers between subjects (19). Between-subject biological variation $\left(\mathrm{CV}_{\mathrm{G}}\right)$ was calculated by the standard ANOVA method (16). Again, the Shapiro-Wilk test was used to verify the normality of the residuals (18).

The RCV values were calculated using the RCV formula as extensively described by Roraas et al. (17). For the RCV calculations in this study, a Z-score of 1.96 was used and the probability level of a significant change was set at $95 \%$.

The index of individuality was calculated following the method of Fraser and Harris (18):

$$
\mathrm{II}=\frac{\sqrt{C V_{A}^{2}+C V_{I}^{2}}}{C V_{G}}
$$

All statistical calculations were performed using SPSS for Windows version 23 (IBM SPSS Statistics, IBM Corporation, Armonk, New York) and Excel 2010.

\section{Results}

One individual (participant 21) was excluded from analysis in advance, since this participant developed a severe cold during the test day and therefore, the clinical situation could not be considered stable.

An overview of the numbers of excluded samples and individuals is given in supplemental table 4.1. In total, $5.9 \%$ of the data was excluded. Since some parameters are posture-dependent and show concentration drops during nighttime (for instance total protein, albumin, homocystein and apolipoprotein A), only daytime data were used to calculate biological variation. 


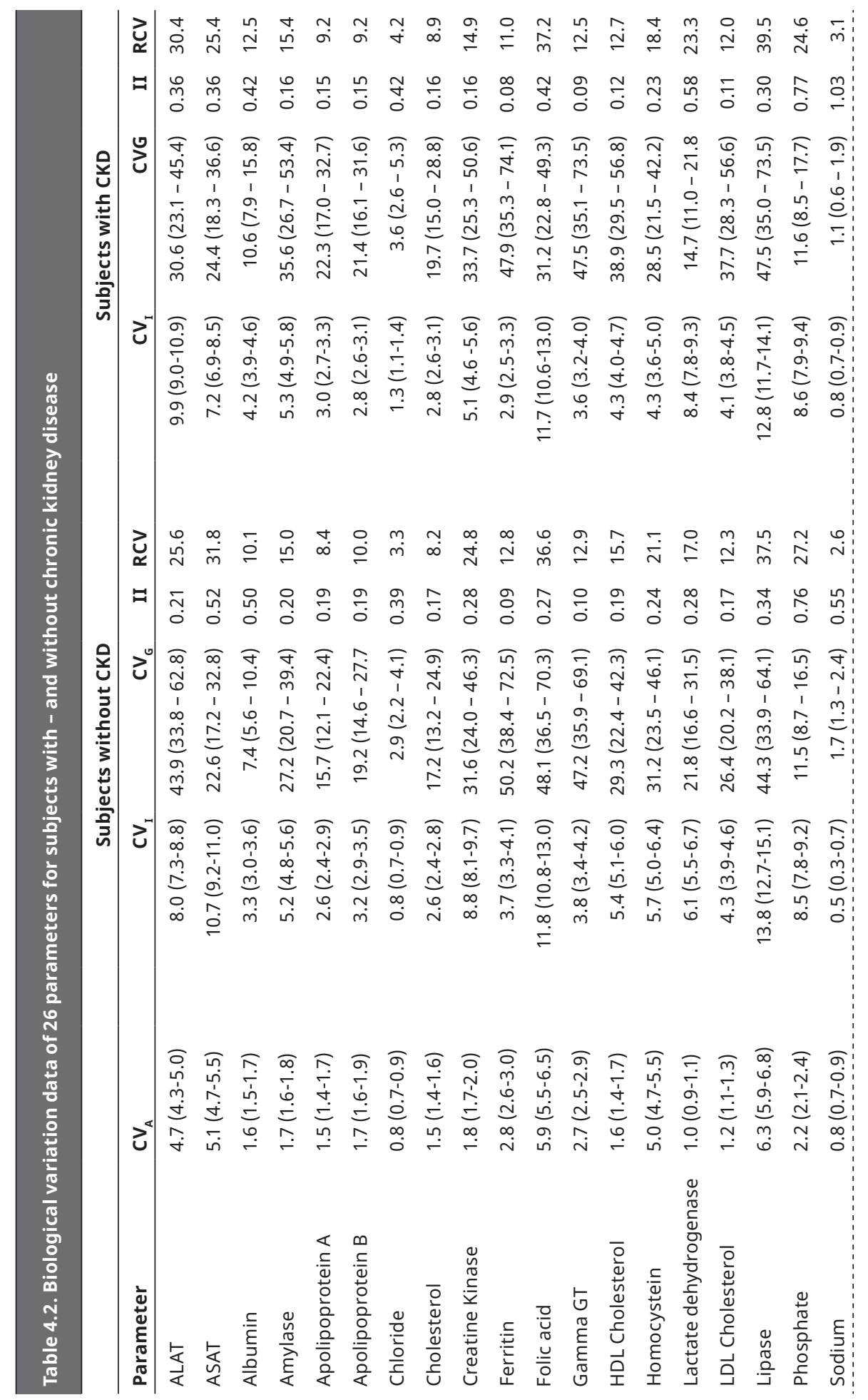




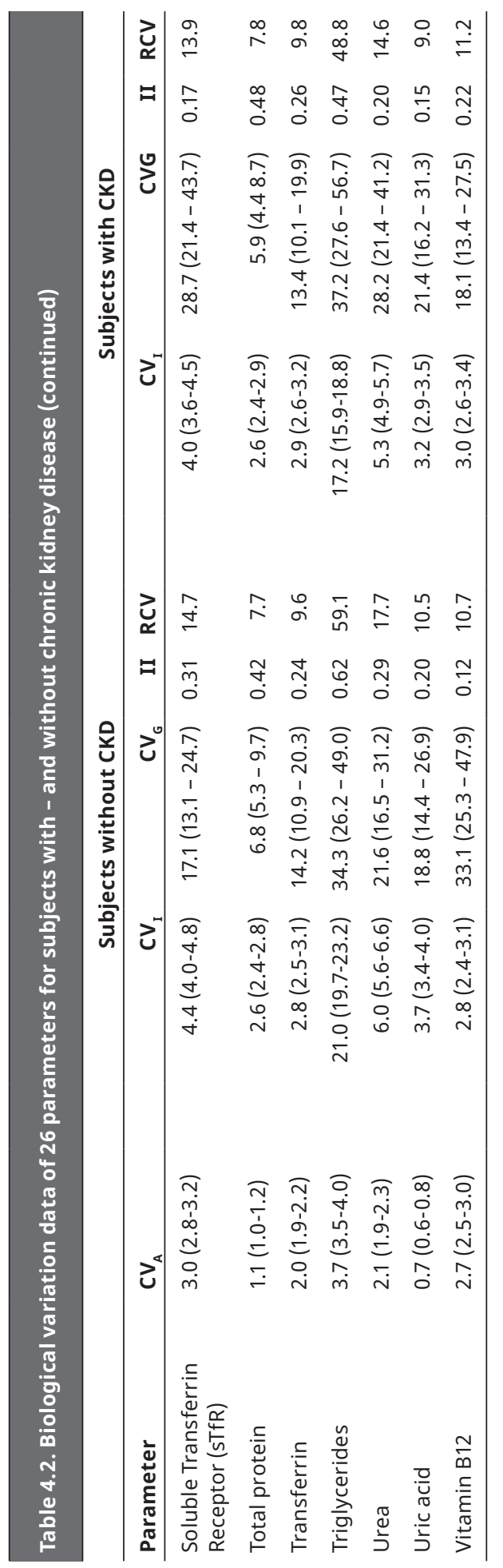


Baseline concentrations were similar for most of the studied parameters (supplemental figures 4.1 and 4.2). No diurnal rhythms were observed in the studied parameters, since we excluded the nighttime values. Some parameter showed some non-rhythmical diurnal variation. However, the observed variation in these parameters was smaller than the corresponding RCV. Therefore, all parameters, except triglycerides, could be considered as having a steady state situation. In triglycerides however, the increase in triglycerides concentration during the day has a physiological reason and can be declared by food consumption.

In table 4.2 , biological variation data of all parameters were shown for subjects with- and without CKD.

$\mathrm{CV}_{\mathrm{G}}$ was larger than $\mathrm{CV}_{\mathrm{I}}$ for all parameters in both study groups, except for sodium and phosphate. $\mathrm{CV}_{\mathrm{I}}$ was similar for the two study groups in 20 parameters (77\%). In the other parameters, $\mathrm{CV}_{\mathrm{I}}$ values differed.

In both study groups, 24 out of 26 parameters (92\%) had an index of individuality (II) <0.6. An II < 0.6 indicates significant individuality which means that, for these parameters, population-based reference values are of limited utility and screening methods using these reference values will not detect (early or latent) disease in many subjects $(8,22)$. The highest II was found for sodium (1.03), which is known for this parameter. $\mathrm{CV}_{\mathrm{A}}$ varied from $0.8 \%$ (sodium and calcium) to $6.3 \%$ (lipase).

In general, RCVs were of similar magnitudes for subjects with- and without CKD. The highest RCVs were found for triglycerides ( $59.1 \%$ and $48.8 \%$, respectively), which can be explained by the strong influence of food consumption on the triglycerides concentration. The lowest RCVs were found for sodium ( $2.6 \%$ and $3.1 \%$, respectively). 
Figure 4.1. 24h variation profiles in people without Chronic Kidney Disease
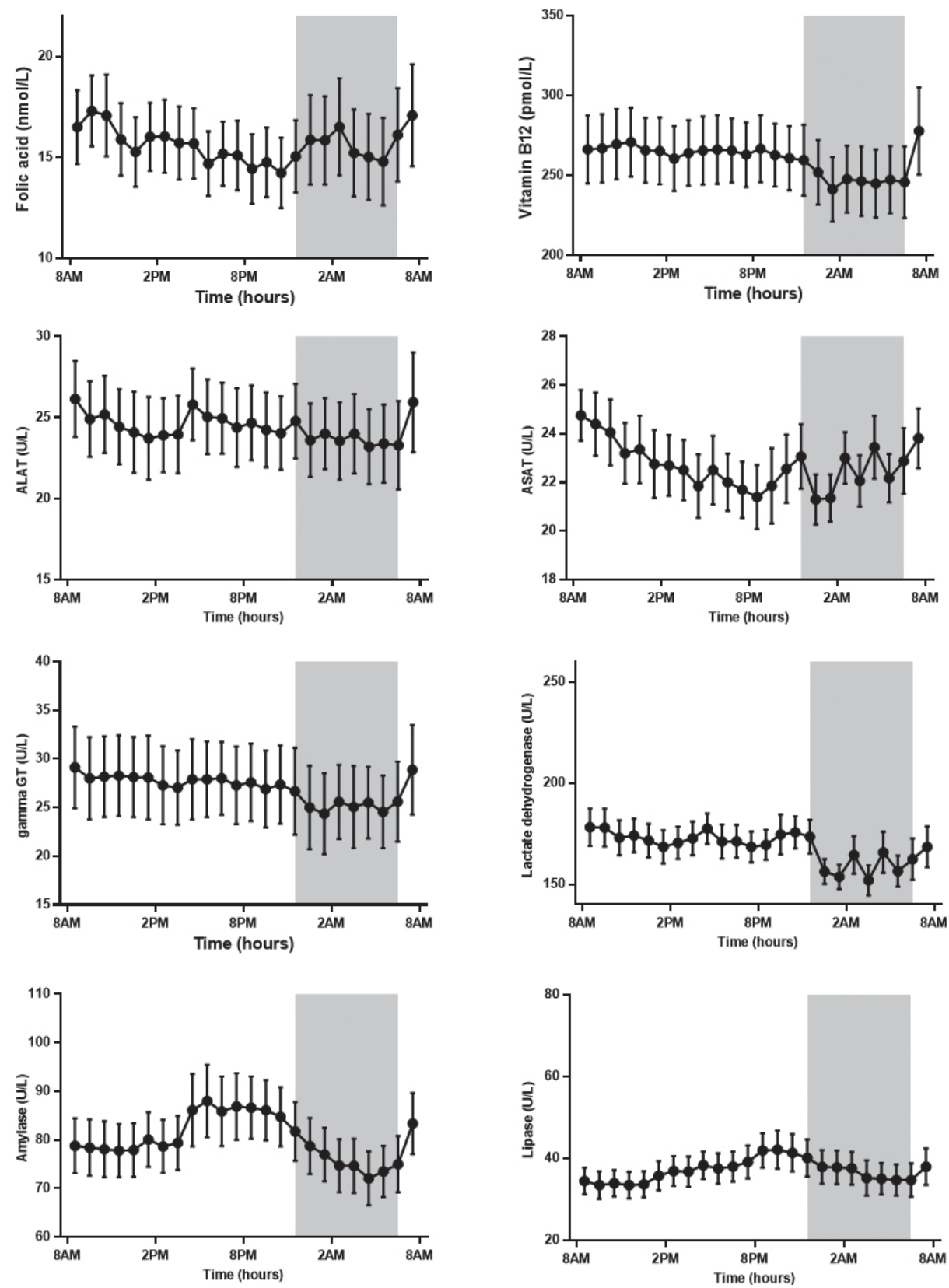

Values represent means \pm SEM. Participants slept between 11:30 PM and 7 AM (shaded area). 
Figure 4.1. 24h variation profiles in people without Chronic Kidney Disease (continued)
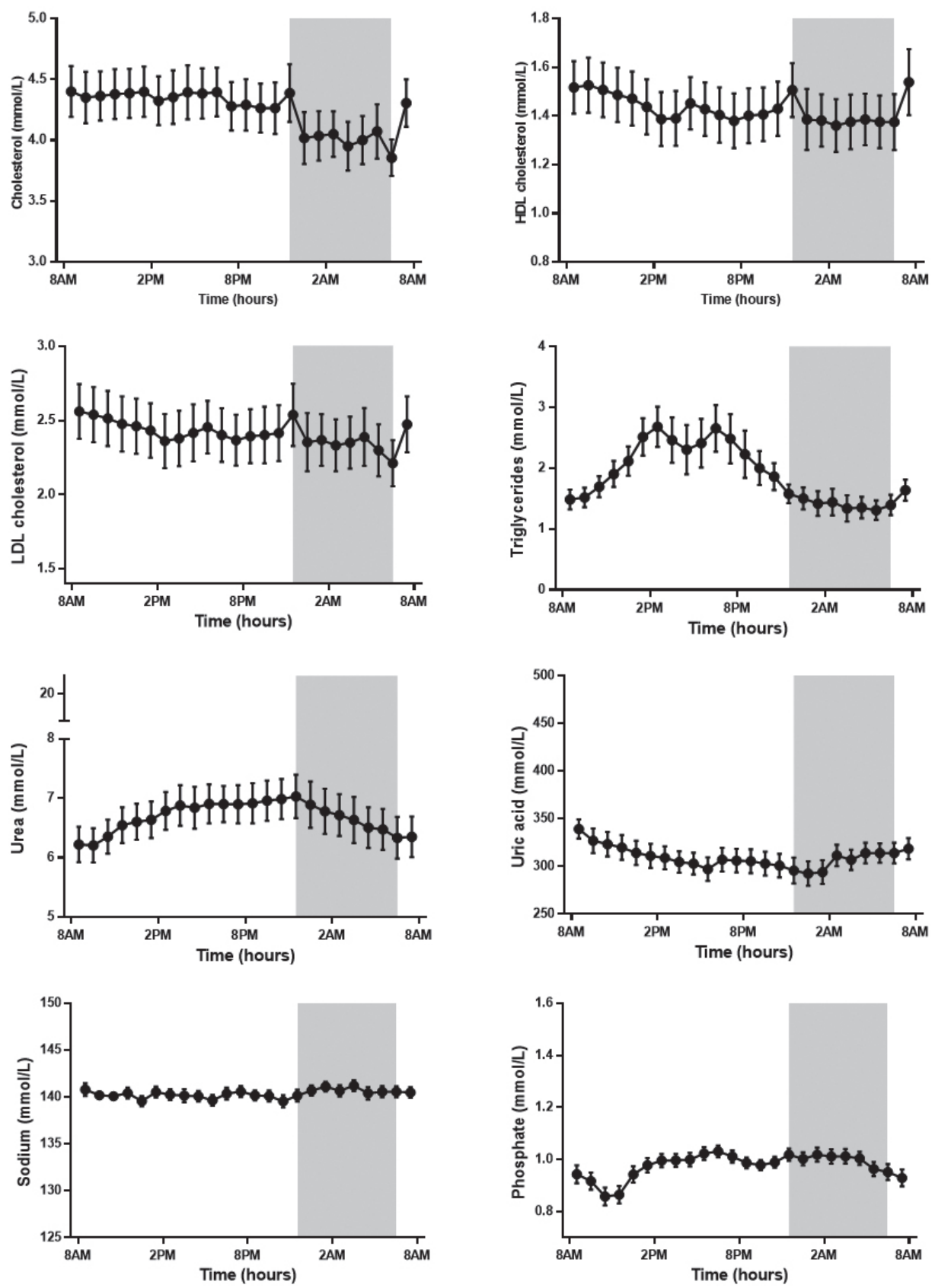

Values represent means \pm SEM. Participants slept between 11:30 PM and 7 AM (shaded area). 
Figure 4.1. 24h variation profiles in people without Chronic Kidney Disease (continued)
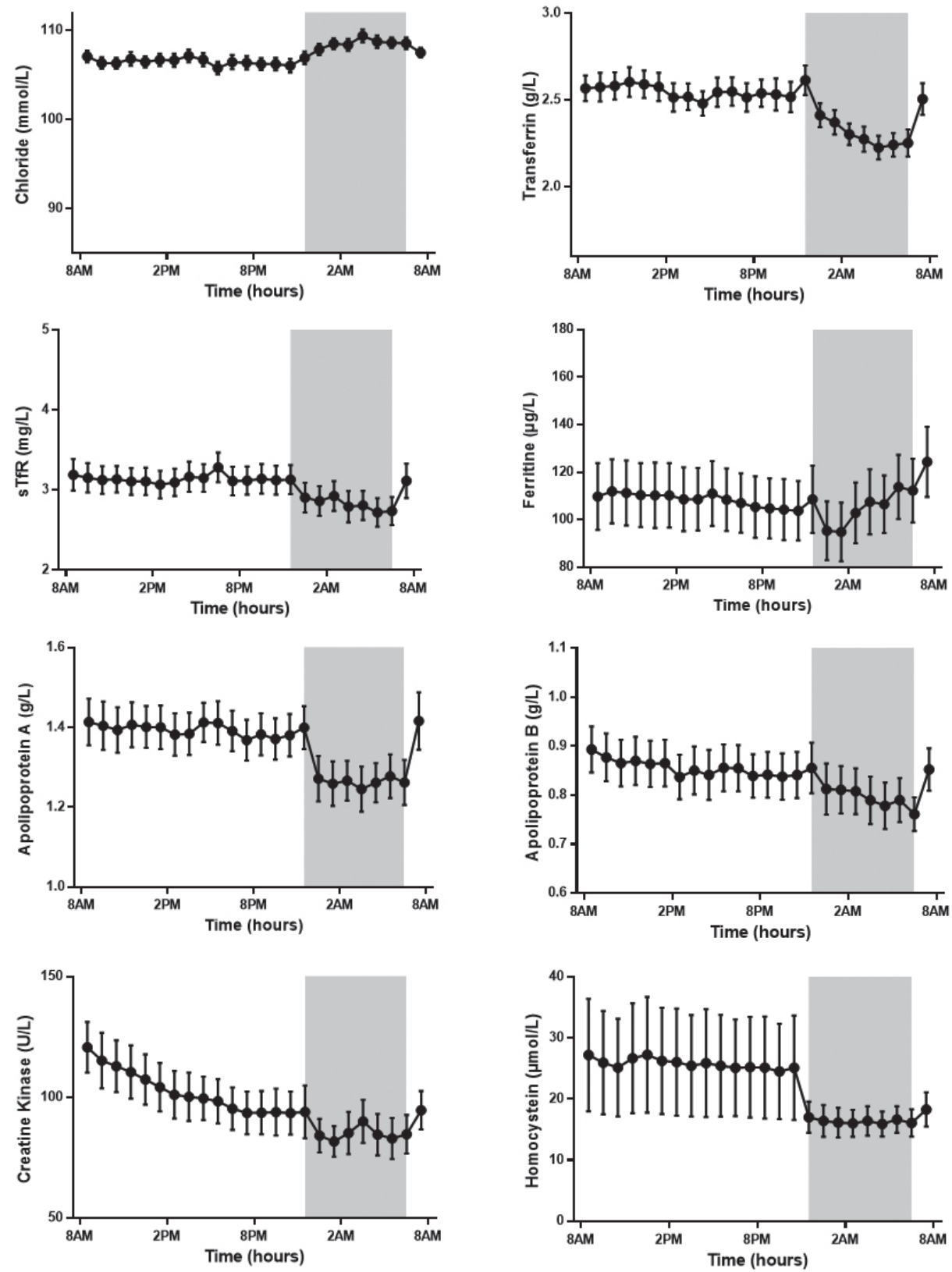

Values represent means \pm SEM. Participants slept between 11:30 PM and 7 AM (shaded area). 
Figure 4.1. 24h variation profiles in people without Chronic Kidney Disease (continued)
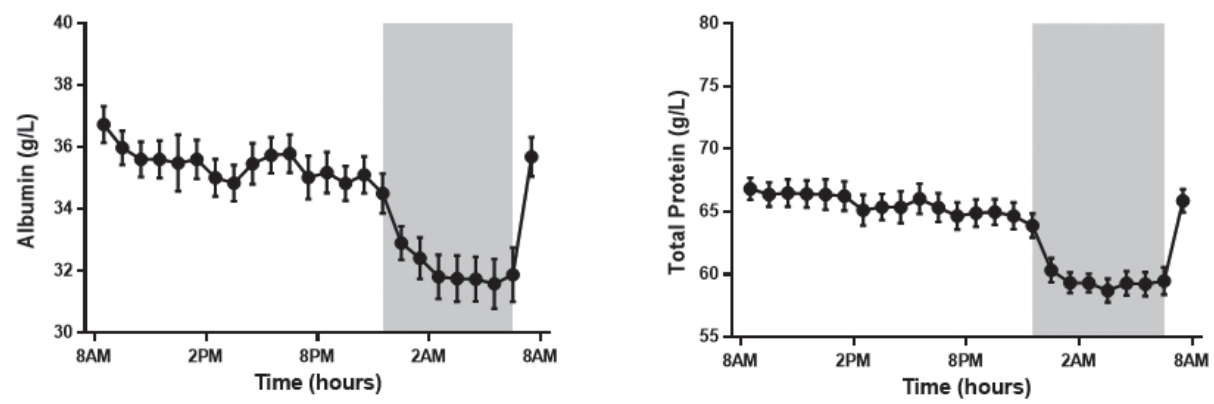

Values represent means \pm SEM. Participants slept between 11:30 PM and 7 AM (shaded area).

\section{Discussion}

In this study we report within-day biological variation data of 26 biochemical parameters. Most of these parameters are frequently ordered from clinical laboratories in daily practice and make an important contribution to diagnosing or monitoring diseases.

This study partially fills the gap in knowledge about within-day biological variation of these parameters.

The main finding in the present study is that the within-subject biological variation $\left(\mathrm{CV}_{\mathrm{I}}\right)$ was found to be of similar magnitude for people with and without CKD for most of the parameters. This is in line with the claim that, for majority of parameters $\mathrm{CV}_{\mathrm{I}}$ values are of the same magnitude in health and stable chronic disease $(9,10)$. For these parameters, physicians do not have to distinguish between diseased and healthy people when they use the $\mathrm{CV}_{\mathrm{I}}$ to interpret consecutive laboratory results. However, for some parameters (for instance creatine kinase and LDH), CV $\mathrm{I}_{\mathrm{I}}$ showed different values between the study groups.

The difference in $\mathrm{CV}_{\mathrm{I}}$ of creatine kinase could be attributed to sex-related differences. Carobene et al. described a higher $\mathrm{CV}_{\mathrm{I}}$ in creatine kinase for men (22). In our study, the group without CKD consisted of $83 \%$ men vs. $70 \%$ in the CKD group. For $\mathrm{LDH}$, the explanation of the higher $\mathrm{CV}_{\mathrm{I}}$ value in the CKD group, is more complicated and could be multifactorial. Factors that have been described to induce diurnal LDH changes are for instance diet, environment temperature and exercise. The RCV values of both CK and LDH however are more similar and we believe these 
biological variation shifts are not of a magnitude that would invalidate the use of RCV's, calculated with $\mathrm{CV}_{\mathrm{I}}$ data from healthy subjects, in these patients.

Since few within-day biological variation studies have been performed, it is not possible to compare our results of all parameters with other studies. However, Bailey et al. report within-day $\mathrm{CV}_{\mathrm{I}}$ values of 17 corresponding parameters (23). The $\mathrm{CV}_{\mathrm{I}}$ values reported in this study were slightly lower than the $\mathrm{CV}_{\mathrm{I}}$ values we found. The most striking difference was STfR, where Bailey et al. reported a $\mathrm{CV}_{\mathrm{I}}$ of $1.4 \%$ and we found CVI values of $4.4 \%$ and $4.0 \%$. However, this study does not seem to be entirely comparable, since it is based on a pediatric population (24).

The $\mathrm{CV}_{\mathrm{I}}$ values reported by Sennels et al. were higher in general, compared to the current study (26). The fact that they found higher $\mathrm{CV}_{\mathrm{I}}$ values may have multiple reasons; the absence of outlier analyses is probably an important one.

Despite the fact that within-day biological variation and between-day biological variation are two different concepts, we have compared our results of some parameters with a between-day biological variation study to get an impression of the magnitude of the differences. Carobene et al. performed a study in which the $\mathrm{CV}_{\mathrm{I}}$ of ALAT, ASAT, gamma GT, LDH, creatine kinase, amylase and lipase was investigated according to the exact same methods as this study (21). The most striking differences were the $\mathrm{CV}_{\mathrm{I}}{ }^{\prime}$ 's of gamma GT (8.9\% vs. 3.8\% and 3.6\%) and creatine kinase (14.5 vs. $8.8 \%$ and $5.1 \%$ ). However, the $\mathrm{CV}_{\mathrm{I}}$ of lipase reported by Carobene et al. was considerably lower (7.7\% vs. $13.8 \%$ and $12.8 \%)$.

Our study differs from existing literature in two important ways. First, the sample collection of most within-day biological variation studies is based on just a few time points $(24,26)$. The calculations in this study were based on 15 consecutive measurements. Second, this study incorporates data from both subjects with and without a chronic illness, which is a positive point compared to many other biological variation studies, which are only performed in healthy subjects. We believe the results of our study are generalizable to the average hospital and outpatient patient and are therefore applicable for the user's clinical practice.

Of course, this study also has some limitations. Unfortunately, it was not possible to stratify biological variation data for gender, since the number of included men and women was unequal. The second limitation concerns the relatively small number 
of participants. Because of the exclusion of outliers, the number of subjects in a group sometimes drops to 16 (in Ferritin for people with CKD). However, outlier exclusion is considered as an absolute requirement for biological variation studies and it is essential that studies on biological variation identify that outlier tests were undertaken as part of the data processing $(2,3,21)$.

In conclusion, variation components from most of the parameters were of the same magnitude in subjects with or without CKD. Our findings imply that the within-day $\mathrm{CV}_{\mathrm{I}}$ is not affected by the presence of chronic (kidney) disease for most biochemical parameters. Thus, the use of RCV's derived from healthy subjects for monitoring patients seems to be reasonable.

Figure 4.2. 24h variation profiles in people with Chronic Kidney Disease
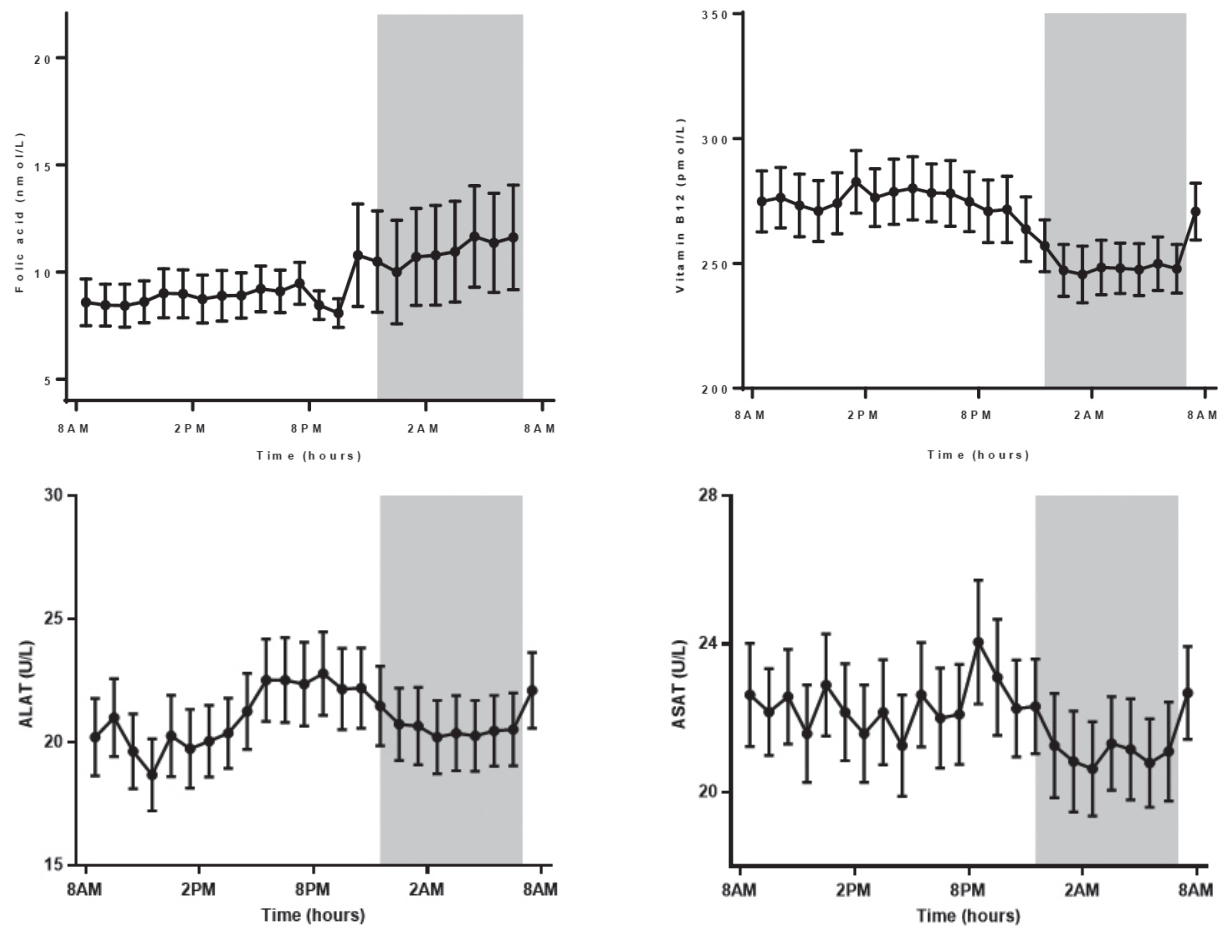

Values represent means \pm SEM. Participants slept between 11:30 PM and 7 AM (shaded area). 
Figure 4.2. 24h variation profiles in people with Chronic Kidney Disease (continued)
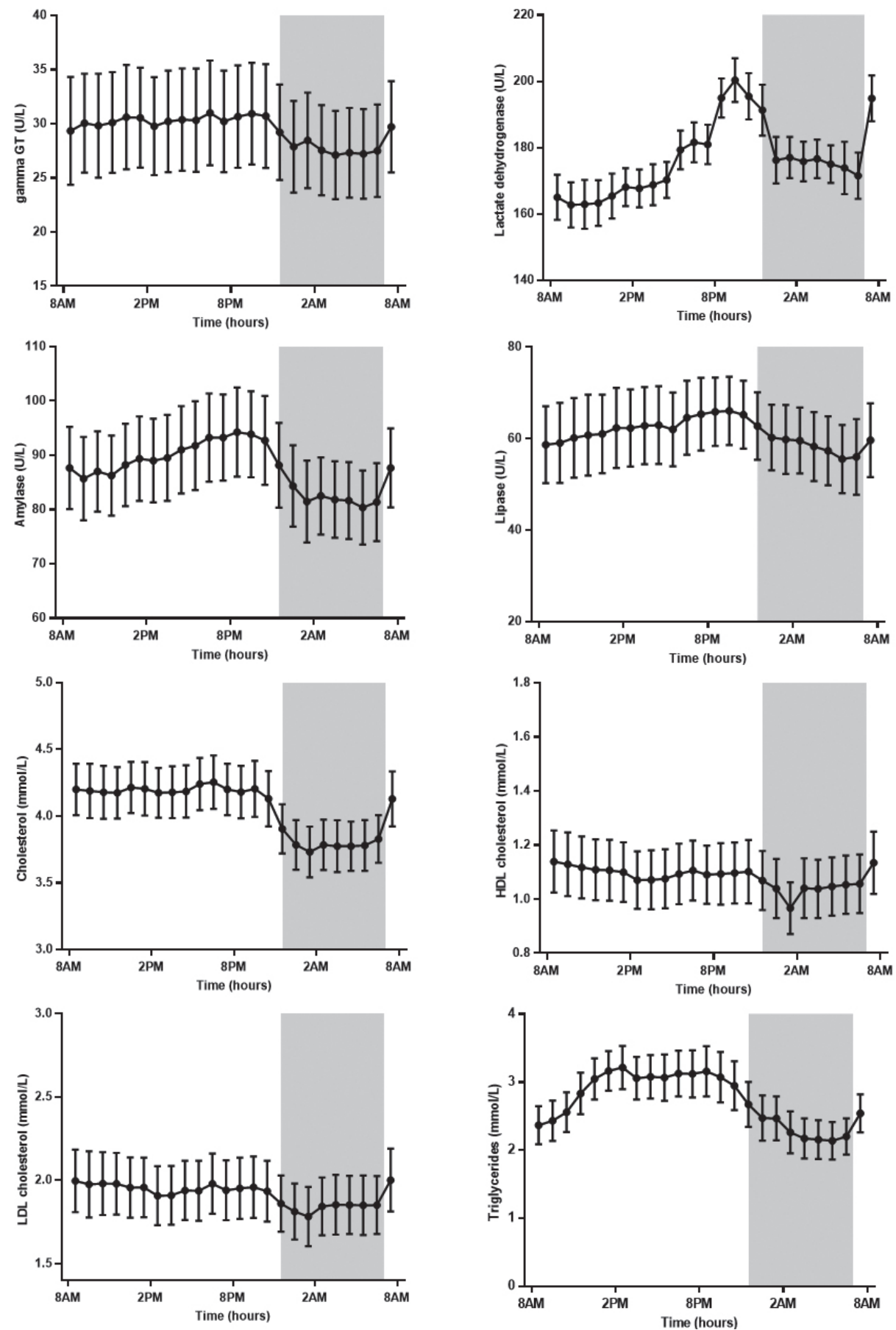

Values represent means \pm SEM. Participants slept between 11:30 PM and 7 AM (shaded area). 
Figure 4.2. 24h variation profiles in people with Chronic Kidney Disease (continued)
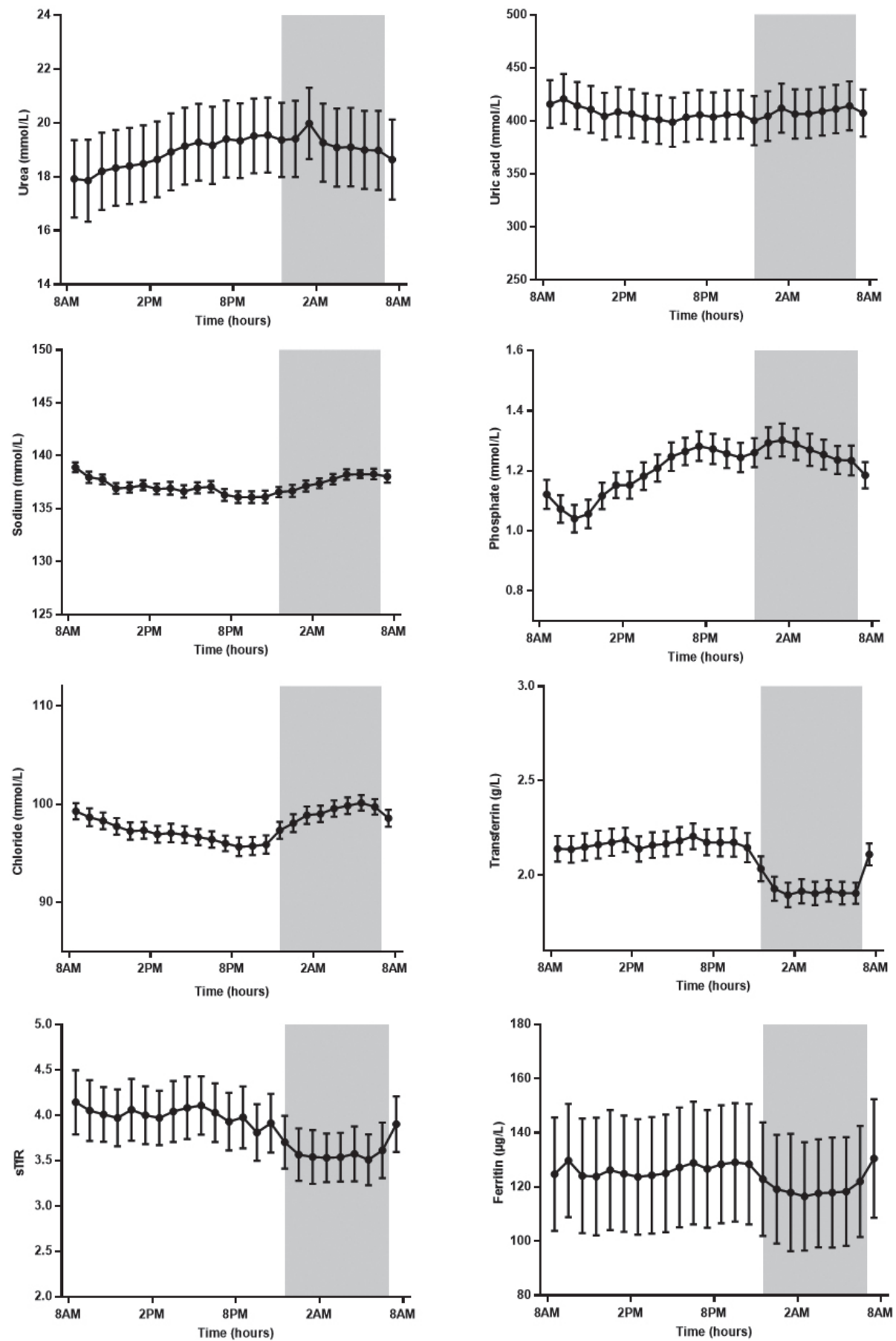

Values represent means \pm SEM. Participants slept between 11:30 PM and 7 AM (shaded area). 
Figure 4.2. 24h variation profiles in people with Chronic Kidney Disease (continued)
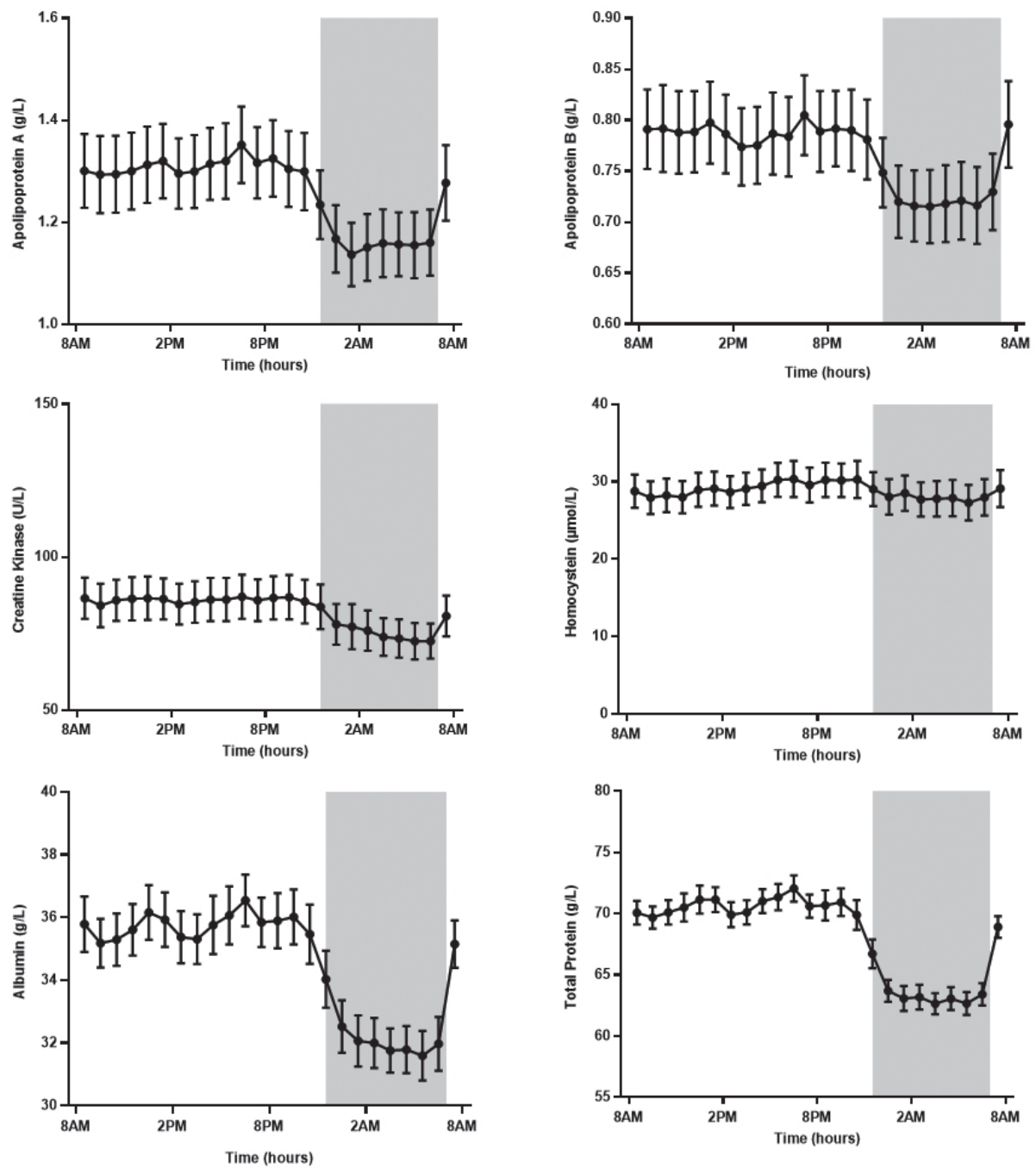

Values represent means \pm SEM. Participants slept between 11:30 PM and 7 AM (shaded area). 


\section{References}

1. Fraser CG. Biological variation: From principles to practice. AACC Press, Washington DC 2001.

2. Aarsand AK, T RR, Fernandez-Calle P, Ricos C, Diaz-Garzon J, Jonker N, et al. The biological variation data critical appraisal checklist:A standard for evaluating studies on biological variation. Clin Chem 2017.

3. Bartlett WA, Braga F, Carobene A, Coskun A, Prusa R, Fernandez-Calle P, et al. A checklist for critical appraisal of studies of biological variation. Clin Chem Lab Med 2015;53:879-85.

4. Carobene A, Strollo M, Jonker N, Barla G, Bartlett WA, Sandberg S, et al. Sample collections from healthy volunteers for biological variation estimates' update: A new project undertaken by the working group on biological variation established by the european federation of clinical chemistry and laboratory medicine. Clin Chem Lab Med 2016;54:1599-608.

5. Pelsers MM, Chapelle JP, Knapen M, Vermeer C, Muijtjens AM, Hermens WT, Glatz JF. Influence of age and sex and day-to-day and within-day biological variation on plasma concentrations of fatty acid-binding protein and myoglobin in healthy subjects. Clin Chem 1999;45:441-3.

6. Winkel $\mathrm{P}$, Statland $\mathrm{BE}$, Bokelund $\mathrm{H}$. The effects of time of venipuncture on variation of serum constituents. Consideration of within-day and day-to-day changes in a group of healthy young men. Am J Clin Pathol 1975;64:433-47.

7. Cotlove E, Harris EK, Williams GZ. Biological and analytic components of variation in long-term studies of serum constituents in normal subjects. 3. Physiological and medical implications. Clin Chem 1970;16:1028-32.

8. Petersen PH, Fraser CG, Sandberg S, Goldschmidt H. The index of individuality is often a misinterpreted quantity characteristic. Clin Chem Lab Med 1999;37:655-61.

9. Ricos C, Iglesias N, Garcia-Lario JV, Simon M, Cava F, Hernandez A, et al. Within-subject biological variation in disease: Collated data and clinical consequences. Ann Clin Biochem 2007;44:343-52.

10. Fraser CG. Biological variation: From principles to practice. Washington DC: AACC Press, 2001.

11. Levey AS, Stevens LA, Coresh J. Conceptual model of ckd: Applications and implications. Am J Kidney Dis 2009;53:S4-16.

12. Roraas T, Petersen PH, Sandberg S. Confidence intervals and power calculations for withinperson biological variation: Effect of analytical imprecision, number of replicates, number of samples, and number of individuals. Clin Chem 2012;58:1306-13.

13. Klinkenberg LJ, Wildi K, van der Linden N, Kouw IW, Niens M, Twerenbold R, et al. Diurnal rhythm of cardiac troponin: Consequences for the diagnosis of acute myocardial infarction. Clin Chem 2016;62:1602-11.

14. Hilderink JM, Klinkenberg LJJ, Aakre KM, de Wit NCJ, Henskens YMC, van der Linden N, et al. Within-day biological variation and hour-to-hour reference change values for hematological parameters. Clin Chem Lab Med 2017;55:1013-24.

15. van der Linden N, Hilderink JM, Cornelis T, Kimenai DM, Klinkenberg LJJ, van Doorn WP, et al. Twenty-four-hour biological variation profiles of cardiac troponin $\mathrm{i}$ in individuals with or without chronic kidney disease. Clin Chem 2017.

16. Hilderink JM, van der Linden N, Kimenai DM, Litjens EJR, Klinkenberg LJJ, Aref BM, et al. Biological variation of creatinine, cystatin c, and egfr over 24 hours. Clin Chem 2018;64:851-60

17. Roraas T, Stove B, Petersen PH, Sandberg S. Biological variation: The effect of different distributions on estimated within-person variation and reference change values. Clin Chem 2016;62:725-36. 
18. Fraser CG, Harris EK. Generation and application of data on biological variation in clinical chemistry. Crit Rev Clin Lab Sci 1989;27:409-37.

19. Shapiro SS, Wilk, M.B. An analysis of variance test for normality (complete samples). Biometrika 1965;52:591-611.

20. Dixon WJ. Processing data for outliers. Biometrics 1953;9:74-89.

21. Inker LA, Schmid CH, Tighiouart H, Eckfeldt JH, Feldman HI, Greene T, et al. Estimating glomerular filtration rate from serum creatinine and cystatin c. N Engl J Med 2012;367:20-9.

22. Carobene A, Roraas T, Solvik UO, Sylte MS, Sandberg S, Guerra E, et al. Biological variation estimates obtained from 91 healthy study participants for 9 enzymes in serum. Clin Chem 2017;63:1141-50.

23. Fraser CG, Wilkinson SP, Neville RG, Knox JD, King JF, MacWalter RS. Biologic variation of common hematologic laboratory quantities in the elderly. Am J Clin Pathol 1989;92:465-70.

24. Bailey D, Bevilacqua V, Colantonio DA, Pasic MD, Perumal N, Chan MK, Adeli K. Pediatric within-day biological variation and quality specifications for 38 biochemical markers in the caliper cohort. Clin Chem 2014;60:518-29.

25. Ricos C, Alvarez V, Perich C, Fernandez-Calle P, Minchinela J, Cava F, et al. Rationale for using data on biological variation. Clin Chem Lab Med 2015;53:863-70.

26. Sennels HP, Jorgensen HL, Fahrenkrug J. Diurnal changes of biochemical metabolic markers in healthy young males - the bispebjerg study of diurnal variations. Scand J Clin Lab Invest 2015;75:686-92. 


\section{Supplemental files}

\section{Supplemental table 4.1. Number of excluded samples per parameter}

\begin{tabular}{|c|c|c|c|c|c|c|}
\hline Parameter & $\begin{array}{r}\text { Analytical } \\
\text { level } \\
\text { (Cochran) }\end{array}$ & $\begin{array}{r}\text { Within- } \\
\text { subject level } \\
\text { (Cochran) }\end{array}$ & $\begin{array}{r}\text { Between- } \\
\text { subject level } \\
\text { (Reed and Dixon) }\end{array}$ & Results & Subjects & $\begin{array}{r}\text { Total } \\
\% \text { of } \\
\text { outliers } \\
\end{array}$ \\
\hline ALAT & 0 & 2 & 0 & 918 & 41 & $7.3 \%$ \\
\hline Albumin & 4 & 2 & 0 & 939 & 41 & $5.2 \%$ \\
\hline Amylase & 0 & 5 & 0 & 822 & 38 & $16.9 \%$ \\
\hline Apolipoprotein A & 3 & 0 & 0 & 987 & 43 & $0.3 \%$ \\
\hline Apolipoprotein B & 1 & 2 & 0 & 917 & 41 & $7.4 \%$ \\
\hline ASAT & 1 & 3 & 0 & 870 & 39 & $12.1 \%$ \\
\hline Chloride & 1 & 0 & 0 & 989 & 43 & $0.1 \%$ \\
\hline Cholesterol & 0 & 3 & 0 & 870 & 40 & $12.1 \%$ \\
\hline Creatine kinase & 1 & 5 & 0 & 845 & 38 & $14.6 \%$ \\
\hline Ferritin & 1 & 6 & 0 & 845 & 35 & $14.6 \%$ \\
\hline Folic acid & 0 & 5 & 1 & 750 & 37 & $24.2 \%$ \\
\hline gamma GT & 0 & 3 & 1 & 846 & 39 & $14.5 \%$ \\
\hline HDL Cholesterol & 0 & 2 & 0 & 894 & 41 & $9.7 \%$ \\
\hline Homocystein & 3 & 2 & 0 & 891 & 41 & $10.0 \%$ \\
\hline LDH & 0 & 2 & 1 & 870 & 41 & $12.1 \%$ \\
\hline LDL Cholesterol & 1 & 1 & 0 & 941 & 42 & $4.9 \%$ \\
\hline Lipase & 1 & 4 & 0 & 869 & 39 & $12.2 \%$ \\
\hline Phosphate & 1 & 3 & 1 & 845 & 39 & $14.6 \%$ \\
\hline Sodium & 0 & 1 & 0 & 942 & 42 & $4.8 \%$ \\
\hline sTfR & 0 & 4 & 0 & 894 & 39 & $9.7 \%$ \\
\hline Total protein & 2 & 0 & 0 & 988 & 43 & $0.2 \%$ \\
\hline Transferrin & 2 & 1 & 0 & 965 & 42 & $2.5 \%$ \\
\hline Triglycerides & 2 & 2 & 0 & 916 & 41 & $7.5 \%$ \\
\hline Urea & 0 & 3 & 0 & 870 & 40 & $12.1 \%$ \\
\hline Uric acid & 0 & 2 & 1 & 870 & 40 & $12.1 \%$ \\
\hline Vitamin B12 & 1 & 1 & 0 & 941 & 42 & $4.9 \%$ \\
\hline
\end{tabular}


Supplemental figure 4.2. 24h variation profiles in people with Chronic Kidney Disease
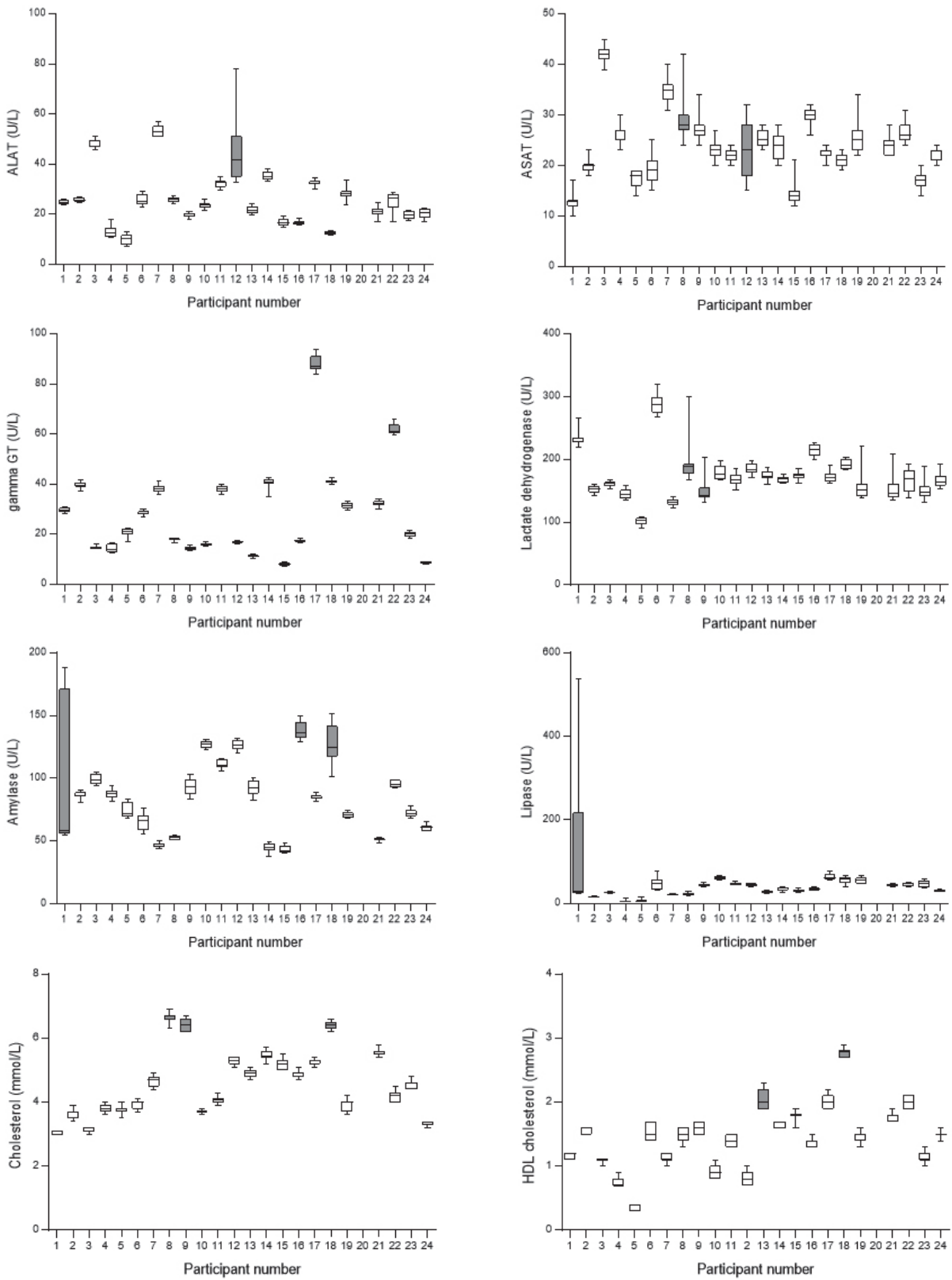

In the box-and whisker plots, the tips of the bars represent the lowest and highest concentrations and the central box covers the 25,50 , and 75 percentiles. Individuals in grey were excluded since they were found to be outliers. 
Supplemental figure 4.2. $24 \mathrm{~h}$ variation profiles in people with Chronic Kidney Disease (continued)
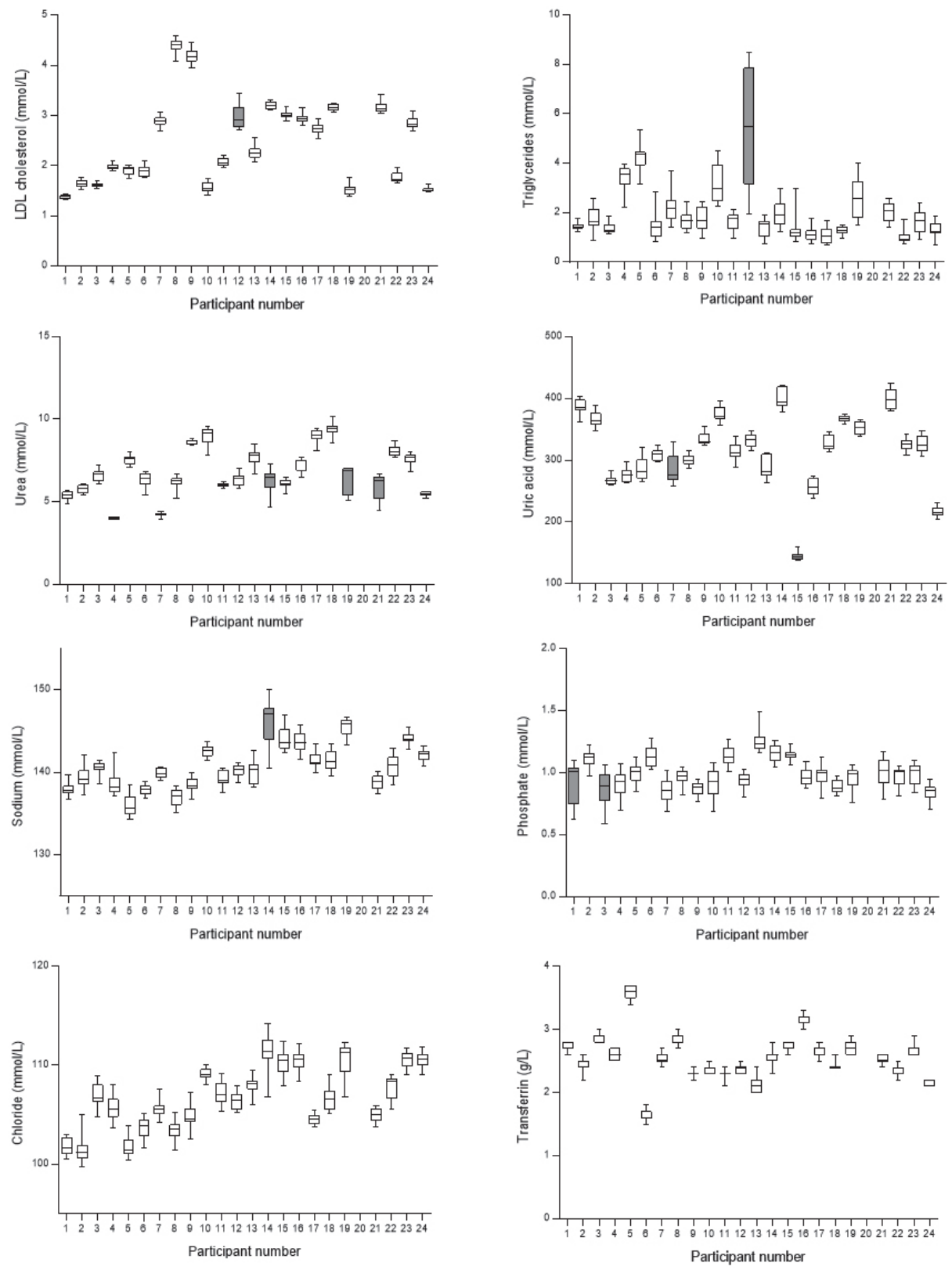

In the box-and whisker plots, the tips of the bars represent the lowest and highest concentrations and the central box covers the 25,50 , and 75 percentiles. Individuals in grey were excluded since they were found to be outliers. 
Supplemental figure 4.2. 24h variation profiles in people with Chronic Kidney Disease (continued)
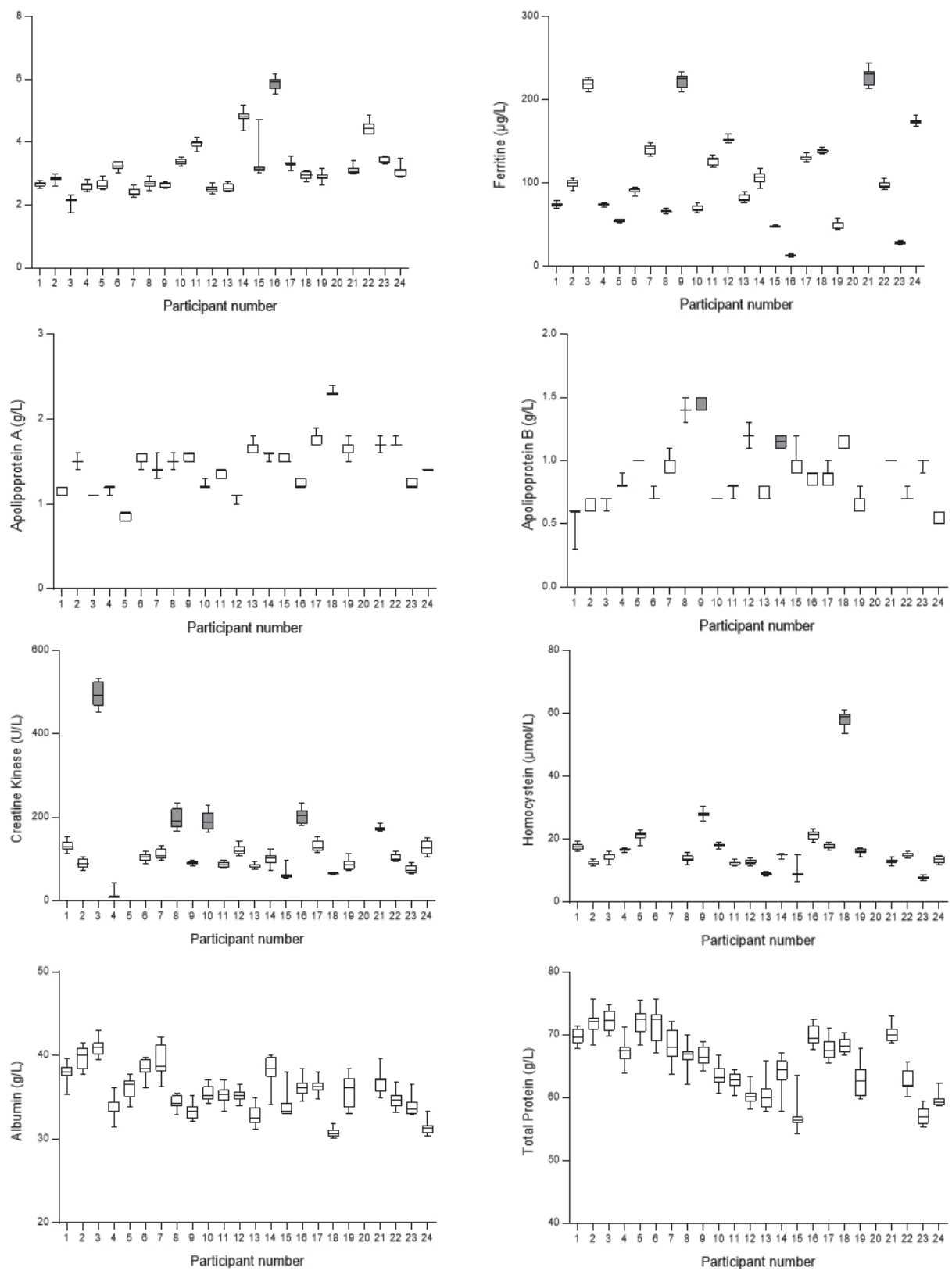

In the box-and whisker plots, the tips of the bars represent the lowest and highest concentrations and the central box covers the 25,50 , and 75 percentiles. Individuals in grey were excluded since they were found to be outliers. 
Supplemental figure 4.2. $24 \mathrm{~h}$ variation profiles in people with Chronic Kidney Disease (continued)
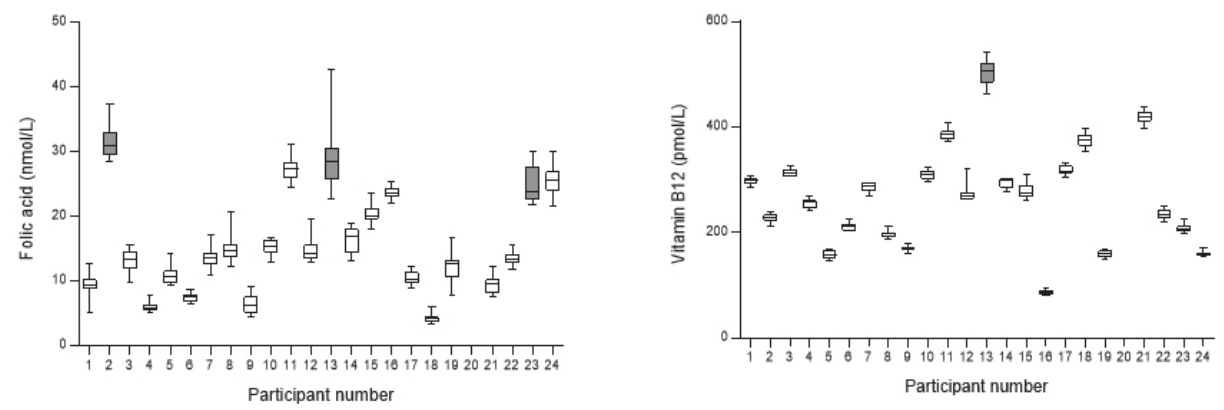

In the box-and whisker plots, the tips of the bars represent the lowest and highest concentrations and the central box covers the 25,50 , and 75 percentiles. Individuals in grey were excluded since they were found to be outliers.

Supplemental figure 4.2 (A-E). Concentration ranges of all parameters for people with CKD
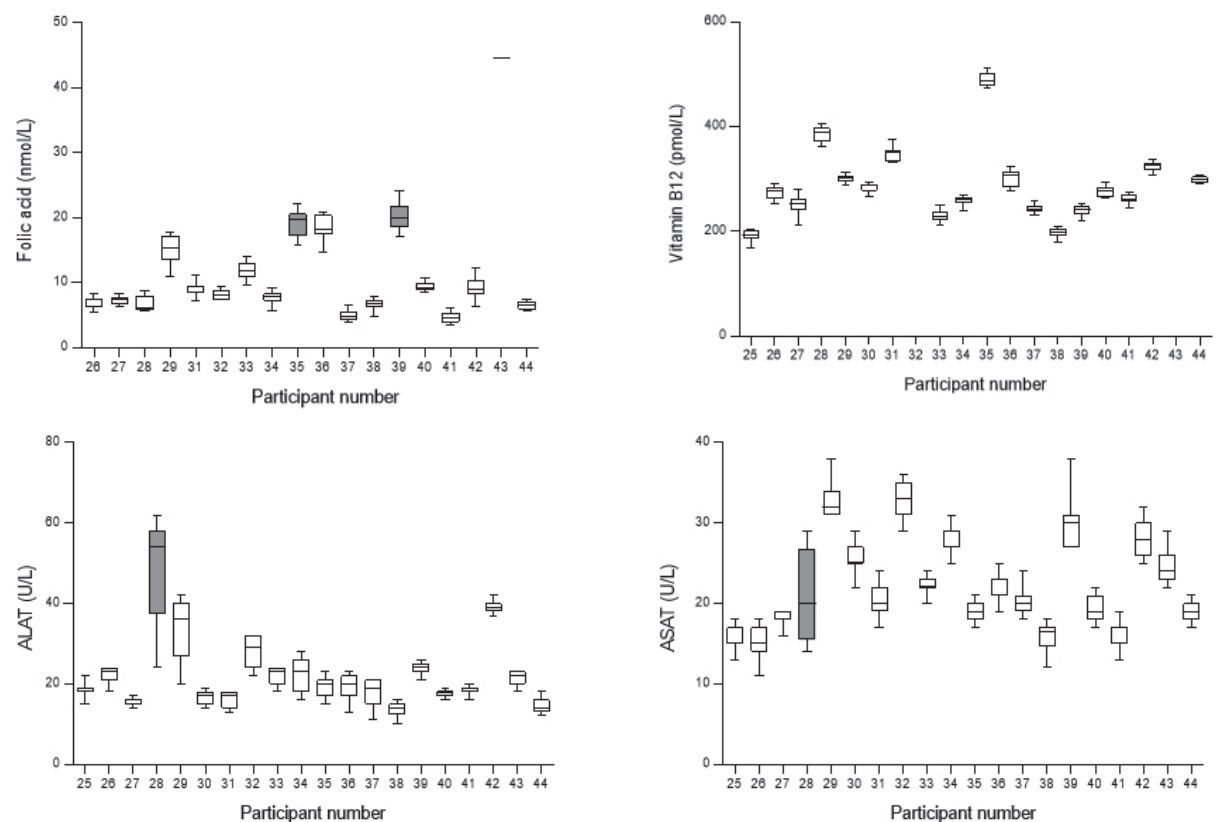

In the box-and whisker plots, the tips of the bars represent the lowest and highest concentrations and the central box covers the 25,50 , and 75 percentiles. Individuals in grey were excluded since they were found to be outliers. 
Supplemental figure 4.2 (A-E). Concentration ranges of all parameters for people with CKD (continued)
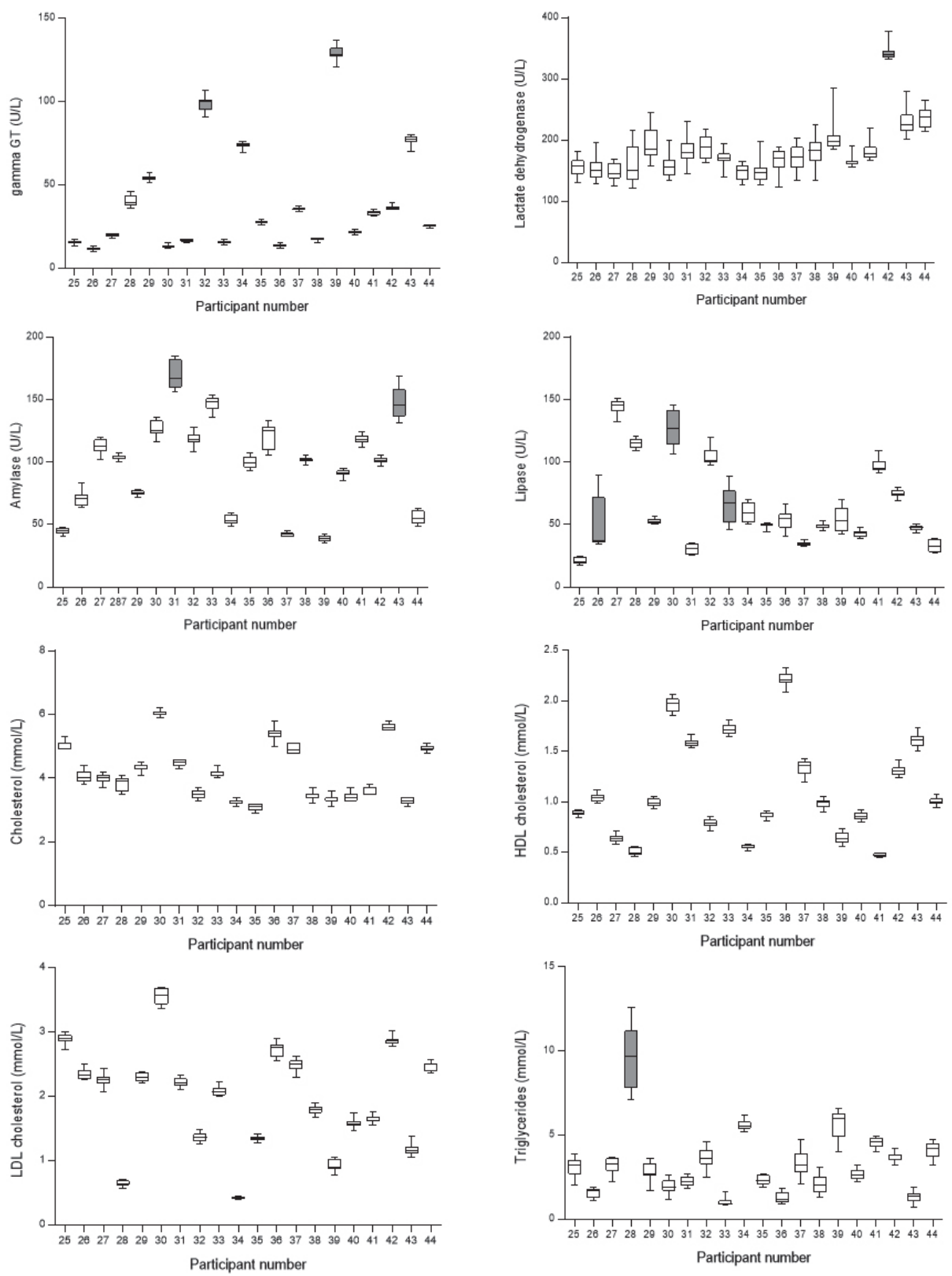

In the box-and whisker plots, the tips of the bars represent the lowest and highest concentrations and the central box covers the 25,50 , and 75 percentiles. Individuals in grey were excluded since they were found to be outliers. 
Supplemental figure 4.2 (A-E). Concentration ranges of all parameters for people with CKD
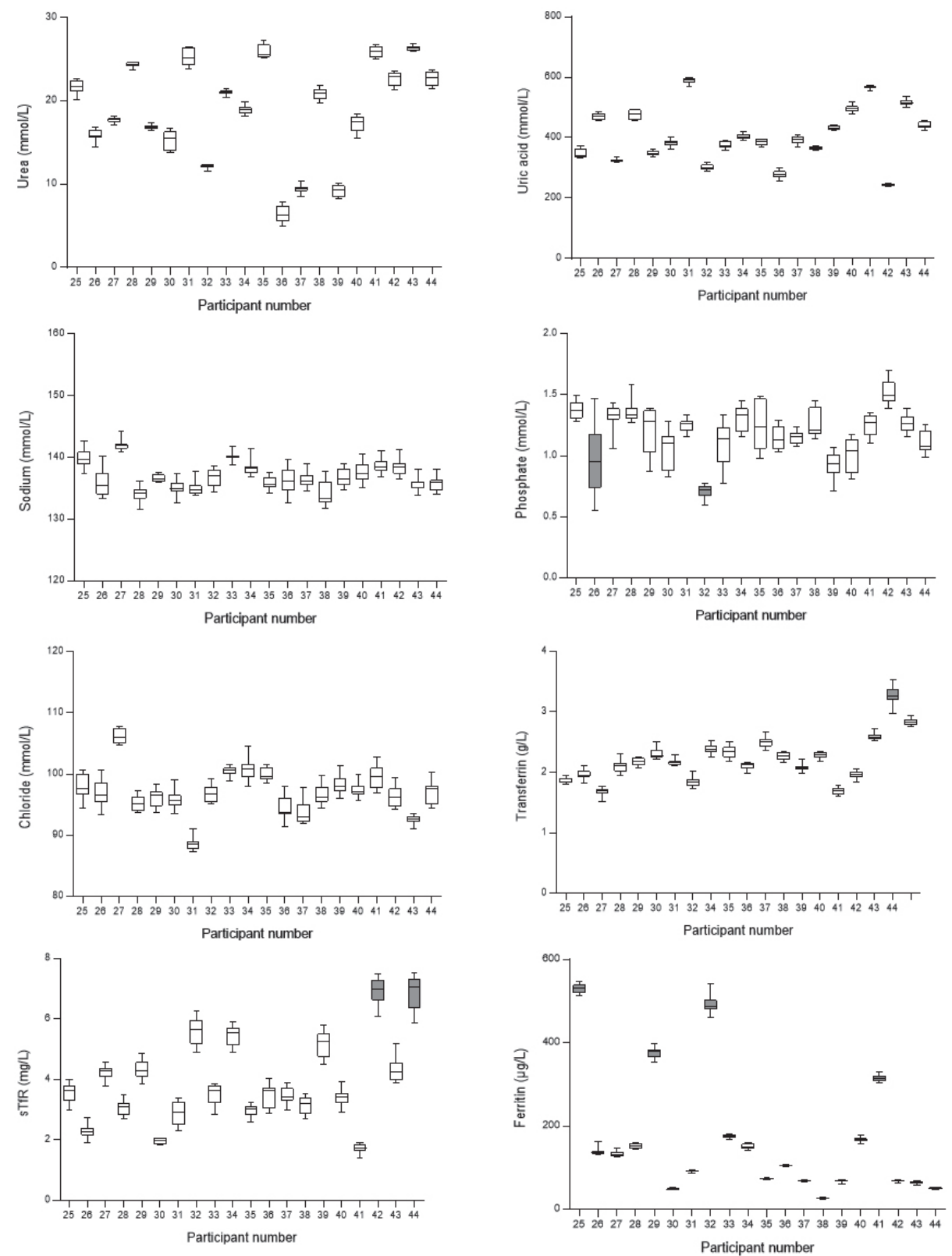

In the box-and whisker plots, the tips of the bars represent the lowest and highest concentrations and the central box covers the 25,50 , and 75 percentiles. Individuals in grey were excluded since they were found to be outliers. 
Supplemental figure 4.2 (A-E). Concentration ranges of all parameters for people with CKD
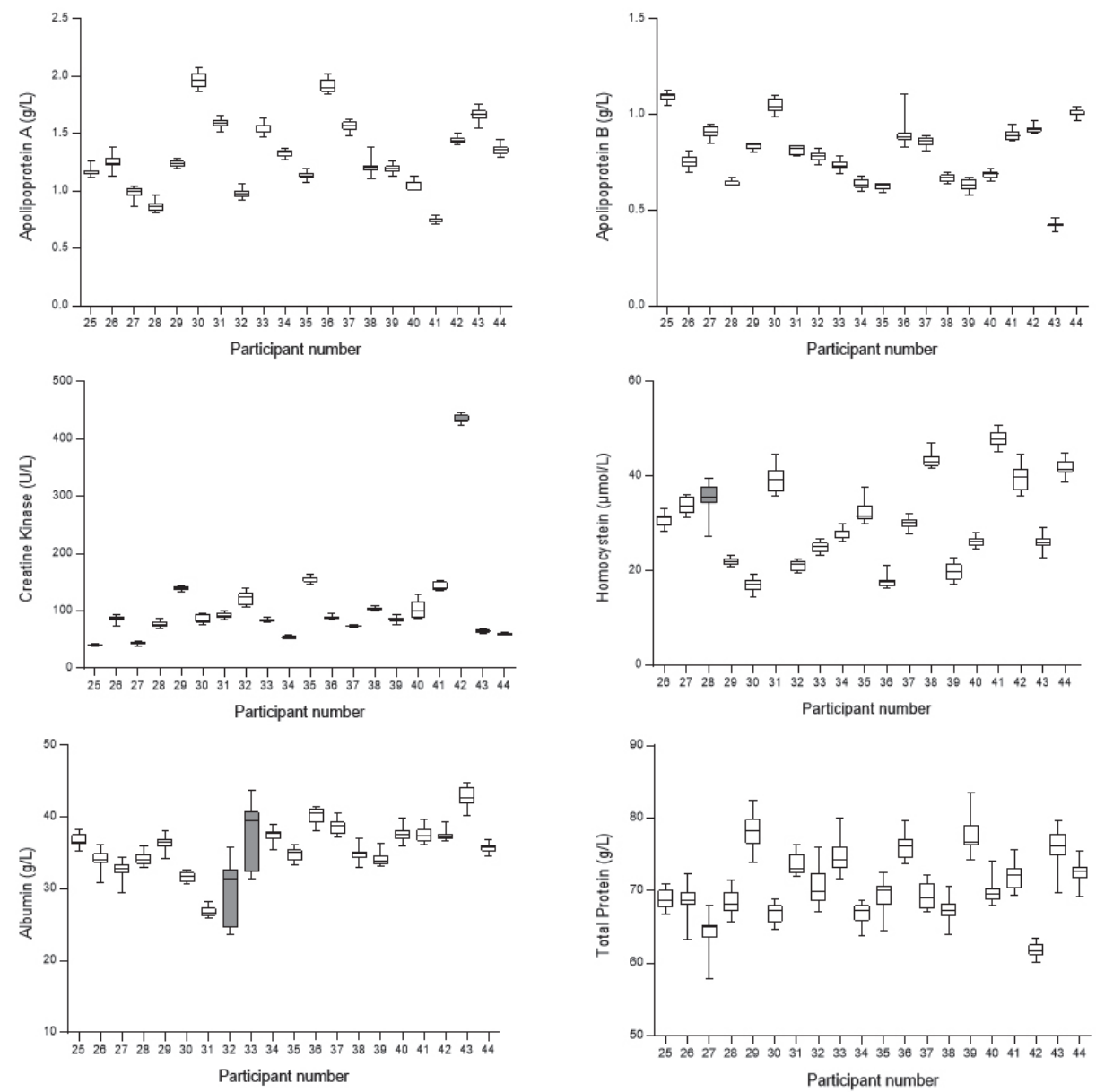

In the box-and whisker plots, the tips of the bars represent the lowest and highest concentrations and the central box covers the 25,50 , and 75 percentiles. Individuals in grey were excluded since they were found to be outliers. 


\section{Chapter 5}




\section{Twenty-four hour biological variation of creatinine, cystatin C and estimated Glomerular Filtration Rate in subjects with or without Chronic Kidney Disease}

J.M. Hilderink, N. van der Linden, D.M. Kimenai , E.J. Litjens, L.J.J. Klinkenberg, B. M. Aref, F. Aziz, J.P. Kooman, R.J.M.W. Rennenberg, O. Bekers, R.P. Koopmans, S.J.R. Meex 


\section{Abstract}

\section{Background}

Estimated glomerular filtration rate (eGFR) is widely used in clinical practice. This study assessed the within-subject biological variation $\left(\mathrm{CV}_{\mathrm{I}}\right)$ of different eGFR equations in people with chronic kidney disease (CKD) and people without CKD. The aims of this study were: 1 ) to determine the $24 \mathrm{~h}$ biological variation profiles of creatinine, cystatin $C$ and eGFR and 2) to determine if $\mathrm{CV}_{\mathrm{I}}$ of creatinine, cystatin $\mathrm{C}$ and eGFR changes upon deterioration of glomerular filtration.

\section{Methods}

Hourly blood samples were analyzed from 37 individuals (17 without CKD, 20 with CKD) during 24h. Creatinine (enzymatic method) and cystatin C were measured using a Cobas 8000 (Roche Diagnostics). eGFR was estimated using the Modification of Diet in Renal Disease (MDRD) and the Chronic Kidney Disease Epidemiology Collaboration (CKD-EPI) based on creatinine and/or cystatin C. Plasma samples were stored at $-80^{\circ} \mathrm{C}$ before analysis. Outlier and homogeneity analyses were checked before performing a nested ANOVA to determine biological variation.

\section{Results}

$\mathrm{CV}_{\mathrm{I}}$ of creatinine was higher in people without CKD than with CKD $(6.4 \%$ versus $2.5 \%$ ) owing primarily to the more profound effect of meat consumption on creatinine variability in individuals with lower baseline creatinine concentrations. Unlike creatinine, cystatin C concentrations were unaffected by meat consumption. Cystatin $C$ showed some diurnal rhythmic variation, less in people with CKD. Reference change values (RCV) of all eGFR equations were within $13-20 \%$ in both study groups.

\section{Conclusion}

Despite differences in $\mathrm{CV}_{\mathrm{I}}$ of creatinine, the $\mathrm{CV}_{\mathrm{I}}$ and RCV of the eGFR equations were relatively similar for people with or without CKD. 


\section{Background}

The estimated glomerular filtration rate (eGFR) is widely applied for the diagnosis of kidney disease and the monitoring of renal function in patients with chronic kidney disease (CKD)(1). CKD is defined as the presence of kidney damage or a glomerular filtration rate (GFR) $<60 \mathrm{~mL} / \mathrm{min} / 1.73 \mathrm{~m} 2$ for three months or longer, irrespective of the cause $(1,2)$. The Chronic Kidney Disease- Epidemiology Collaboration equation (CKD-EPI) and the Modification of Diet in Renal Disease (MDRD) are the equations most commonly used in clinical practice to estimate the GFR $(3,4)$. Although the equations are constructed differently, the calculation of both CKD-EPI and MDRD is based on the two renal biomarkers: creatinine and / or cystatin C (3-5). Serum creatinine and cystatin C concentrations can fluctuate during the day, either from 'true' circadian differences in GFR or from random biological variation, without reflecting 'real' changes $(6,7)$. These fluctuations could affect the interpretation of eGFR values based on creatinine and / or cystatin C. Insight regarding the magnitude of diurnal fluctuations may prevent physicians from erroneously interpreting random variations as clinically relevant changes (8).

Biological variation studies allow a systematic assessment of random diurnal variation of biomarkers. Thus far, most studies that have assessed the biological variation of renal biomarkers and eGFR reported on between-day variation (6, 9-17), but data on within-day biological variation are scarce (18). Moreover, most studies focused either on healthy volunteers, or on people with impaired renal function, but data from a direct comparison of people with and without CKD are not available $(6,15,19,20)$. In this study, we aimed to construct $24 \mathrm{~h}$ profiles of creatinine and cystatin C concentrations, as well as GFR estimations that are based on creatinine and/ or cystatin C. In addition, we assessed whether variation over the day is of similar magnitude in people with CKD and people without CKD.

\section{Materials and methods}

\section{Study design and population}

This study on $24 \mathrm{~h}$ variability in renal function and eGFR was performed between January 2013 and October 2015, as described previously (21, 22). It conformed to the principles of the Declaration of Helsinki (23) and was approved by the institutional review board and the ethics committee at Maastricht University Medical Center (MUMC). Each participant provided written informed consent. 
The design of this study complied with the current checklists regarding biological variation as much as possible $(24,25)$.

In total 44 individuals were included, and divided into two study groups: The first study group consisted of 24 individuals without clinically diagnosed CKD (79\% males and $21 \%$ females), and the second group ( $70 \%$ males and $30 \%$ females) consisted of 20 patients (subject number 25 - 44) with clinically diagnosed CKD stage 3 or higher (eGFR $<60 \mathrm{~mL} / \mathrm{min} / 1.73 \mathrm{~m}^{2}$ ) (2). This number of individuals per group afforded sufficient power to make reliable estimations about biological variation (26). All individuals were Caucasian and aged between $39-83$ years. Diabetes was defined as fasting plasma glucose $\geq 126 \mathrm{mg} / \mathrm{dL}$ and/or $\mathrm{HbA1c} \geq 6.5 \%$ (=47 mmol/mol) (27). Exclusion criteria were: current dialysis treatment, an acute myocardial infarction (AMI) in the 12 months prior to the study, active cardiac disease (angina pectoris, cardiomyopathy or myocarditis), and anemia (hemoglobin $<10.5 \mathrm{~g} / \mathrm{dL}$ ).

\section{Table 5.1. Subject's baseline characteristics}

\begin{tabular}{|c|c|c|}
\hline & $\begin{array}{l}\text { Subjects without CKD } \\
(n=17)\end{array}$ & $\begin{array}{l}\text { Subjects with CKD } \\
(n=20)\end{array}$ \\
\hline Age (years) & $72 \pm 7$ & $66 \pm 12$ \\
\hline Male sex & $14(82)$ & $14(70)$ \\
\hline Body mass index $\left(\mathrm{kg} / \mathrm{m}^{2}\right)$ & $26 \pm 3$ & $28 \pm 4$ \\
\hline Diabetes Mellitus ${ }^{a}$ & $6(35)$ & $7(35)$ \\
\hline Cholesterol concentration (mg/dL) & $176 \pm 35$ & $157 \pm 33$ \\
\hline Systolic blood pressure $(\mathrm{mmHg})$ & $140 \pm 15$ & $136 \pm 19$ \\
\hline Diastolic blood pressure $(\mathrm{mmHg})$ & $68 \pm 8$ & $86 \pm 14$ \\
\hline Creatinine (mg/dL) & $1.0 \pm 0.2$ & $3.3 \pm 1.0$ \\
\hline Cystatin C (mg/L) & $1.0 \pm 0.2$ & $2.8 \pm 0.8$ \\
\hline $\operatorname{MDRD}\left(\mathrm{ml} / \mathrm{min} / 1.73 \mathrm{~m}^{2}\right)$ & $73.4 \pm 18.5$ & $19.2 \pm 6.4$ \\
\hline CKD-EPI ${ }_{\text {creatinine }}\left(\mathrm{ml} / \mathrm{min} / 1.73 \mathrm{~m}^{2}\right)$ & $72.9 \pm 17.2$ & $18.9 \pm 6.6$ \\
\hline CKD-EPI ${ }_{\text {cystatin C }}\left(\mathrm{ml} / \mathrm{min} / 1.73 \mathrm{~m}^{2}\right)$ & $74.2 \pm 17.2$ & $20.2 \pm 8.5$ \\
\hline CKD-EPI creatinine - cystatin C $\left(\mathrm{ml} / \mathrm{min} / 1.73 \mathrm{~m}^{2}\right)$ & $74.2 \pm 17.2$ & $19.0 \pm 7.0$ \\
\hline Chronic Kidney Disease & $0(0)$ & $20(100)$ \\
\hline Glomerular disease & NA & $7(35)$ \\
\hline Tubulointerstitial disease & NA & $4(20)$ \\
\hline Vascular disease & NA & $8(40)$ \\
\hline Cystic and congenital disease & NA & $1(5)$ \\
\hline
\end{tabular}

Continuous data is presented as mean \pm SD and categorical data is presented as $n(\%)$ a Diabetes was defined as fasting plasma glucose $\geq 126 \mathrm{mg} / \mathrm{dL}$ and $/$ or $\mathrm{HbA} 1 \mathrm{c} \geq 6.5 \%(=47 \mathrm{mmol} / \mathrm{mol})(27)$ NA, not applicable. SD, standard deviation. 


\section{Sample collection and handling}

Creatinine and cystatin C were measured on a Cobas ${ }^{\circledR} 8000$ (Roche Diagnostics) using the following Roche reagents: CREP2, Creatinine plus ver.2, code 05168589 and CYSC2, Tina-quant Cystatin C Gen.2, code 06600239. We used an enzymatic method to measure creatinine, based on the conversion of creatinine with the aid of creatininase, creatinase and sarcosine oxidase to glycine, formaldehyde and hydrogen peroxide. We used Bio-rad controls for creatinine and 'Cystatin C Control Set Gen. 2' for cystatin C (Roche Diagnostics).

The eGFR was calculated using MDRD and CKD-EPI formulae $(3,5)$. Three CKD-EPI estimations were calculated: CKD-EPI creatinine, CKD-EPI cystatin C, and CKD-EPI creatinine-cystatin C (4).

Hourly blood samples were drawn in ethylenediaminetraacetic acid (EDTA)containing tubes $(8 \mathrm{ml})$. The plasma samples were centrifuged immediately (centrifugation at $2700 \mathrm{~g}$ for 12 minutes at room temperature) after collection and plasma was stored at $-80^{\circ} \mathrm{C}$ until analysis. The plasma samples were then thawed and all samples from the same individual were analyzed within a single run.

To estimate analytical variation $\left(\mathrm{CV}_{\mathrm{A}}\right)$, samples from eight randomly selected individuals (four individuals without CKD and four individuals with CKD, 18\% of all samples) were analyzed in duplicate.

\section{Study procedure}

To study the within-day biological variability, blood samples were drawn every hour during $24 \mathrm{~h}$ using an intravenous cannula. The participants arrived at 8:00 $\mathrm{AM}$ at the laboratory after an overnight fast. During the test day, participants were restricted to the laboratory under sedentary conditions. Mealtimes were standardized at 8:30 AM (breakfast), 12:30 AM (lunch) and 6:00 PM (dinner). The participants could select from approximately four dinner options. The majority of dinner options consisted of dishes containing meat. The meal options and meat content were not standardized. Breakfast and lunch consisted of sandwiches with cheese, ham or sweet toppings. The participants went to bed at 11:30 PM and got up the next morning at 7:00 AM. During the night, an extension line was attached to the cannula to prevent sleep disturbance during blood sampling. 


\section{Statistical analysis}

As recommended for biological variation studies, we performed outlier analyses on three levels (analytical, within-subject and between-subject) $(7,26)$. The Cochran test was used to identify outliers on the analytical and within-subject level and the Dixon-Reed criterion was performed to identify outliers on the betweensubject level $(7,8,28,29)$. People with outlying within-subject variances were rejected from calculations, due to their heterogeneity of variance (24). Since data on the analytical, within-subject and between-subject levels followed a Gaussian distribution (Shapiro-Wilk test), transformation of the data was not required. For the analytical normality check, we used data of eight individuals which were measured in duplicate (192 replicates). For the within-subject level we verified normality for each individual subject. For the between-subject level, we verified normality for the means of each individual. The variation on three levels, the between-subject variation $\left(\mathrm{CV}_{\mathrm{G}}\right)$, within-subject biological variation $\left(\mathrm{CV}_{\mathrm{I}}\right)$, and $\mathrm{CV}_{\mathrm{A}}$ were calculated using a nested ANOVA with $95 \%$ confidence intervals determined according to the method of Burdick and Graybill $(8,26,30)$.

Reference change values (RCVs) (Z-score of 1.96) and the index of individuality (II) were calculated according to the method of Fraser and Harris (8):

$$
\begin{gathered}
R C V=\sqrt{2} * Z * \sqrt{C V_{A}^{2}+C V_{I}^{2}} \\
\mathrm{II}=\frac{\sqrt{C V_{A}^{2}+C V_{I}^{2}}}{C V_{G}}
\end{gathered}
$$

We used an F-test to monitor whether the degree of renal impairment was stable in the people with CKD. To investigate this, we examined the eGFR one week, one month, and three months after the initial test day. P-values $<0.05$ were considered statistically significant.

To visualize the diurnal rhythm of cystatin C, $24 \mathrm{~h}$ concentration curves were fitted for both study groups using cosinor rhythmometry.

All statistical calculations were performed using SPSS for Windows version 23 (IBM SPSS Statistics, IBM Corporation). 


\section{Results}

Eligibility of participants

A total of 44 individuals, 24 without CKD and 20 with CKD, participated in this biological variation study. Two participants ( 1 and 8 ) left the study prematurely and two other participants (15 and 23) had missing data due to problems with the venous cannula overnight. In order to maintain a balanced design, and in line with the statistical conditions for a nested ANOVA, these people were considered 'not eligible' and excluded from further analyses (31). Additionally, participant 20 was excluded, because this participant developed a severe cold during the test day and therefore, the clinical situation of this participant was unstable. Although participants 10 and 18 were not clinically diagnosed with CKD, these participants were excluded because the creatinine concentrations were not appropriate for the non-CKD group (mean concentrations $1.8 \pm 0.2 \mathrm{mg} / \mathrm{dL}$ and $2.0 \pm 0.08 \mathrm{mg} / \mathrm{dL}$, respectively). In online Supplemental Figure 5.1, a flowchart of the study was shown. Eventually, 37 individuals were considered eligible for analyses. Baseline characteristics of the two study groups are shown in Table 5.1.

\section{Clinical stability of people with CKD}

To verify that the people with CKD were clinically stable and not rapidly progressive in terms of their CKD, additional blood samples were taken at one week, one month, and three months after the test day at the same time as the baseline measurement on the initial test day (08:30 AM). The eGFR values (calculated with MDRD, CKD-EPI creatinine, CKD-EPI cystatin C, and CKD-EPI creatinine - cystatin C) did not decrease significantly during this follow-up period (min-max follow-up concentration ranges: MDRD $18.7-19.3 \mathrm{~mL} / \mathrm{min} / 1.73 \mathrm{~m} 2, \mathrm{p}=0.63$; CKD-EPI creatinine 18.3 - 18.9 $\mathrm{mL} / \mathrm{min} / 1.73 \mathrm{~m} 2, \mathrm{p}=0.99$; CKD-EPI cystatin C $20.1-20.3 \mathrm{~mL} / \mathrm{min} / 1.73 \mathrm{~m} 2, \mathrm{p}=0.49$; CKD-EPI creatinine-cystatin C 18.6 - $19.0 \mathrm{~mL} / \mathrm{min} / 1.73 \mathrm{~m} 2, \mathrm{p}=0.99)$. Therefore, the participants with CKD were considered as having a stable chronic disease. 
Table 5.2. Components of biological variation, indexes of individuality and reference change values for renal biomarkers and different eGFR equations in both study groups

\begin{tabular}{lllllll}
$n$ & Mean & $\mathrm{CV}_{\mathrm{A}}$ & $\mathrm{CV}_{\mathrm{I}}$ & $\mathrm{CV}_{\mathrm{G}}$ & II & $\mathrm{RCV}$ \\
\hline
\end{tabular}

\section{Subjects without CKD}

\begin{tabular}{|c|c|c|c|c|c|c|c|}
\hline Creatinine & 16 & $1.0 \mathrm{mg} / \mathrm{dL}$ & $1.1(1.0-1.3)$ & $6.4(6.0-6.9)$ & $21.2(15.7-32.9)$ & 0.3 & 18.2 \\
\hline Cystatin C & 17 & $1.0 \mathrm{mg} / \mathrm{dL}$ & $1.1(1.0-1.3)$ & $4.1(3.8-4.4)$ & $15.3(11.3-23.2)$ & 0.3 & 11.6 \\
\hline MDRD & 16 & $\begin{array}{l}75.3 \mathrm{~mL} / \\
\mathrm{min} / 1.73 \mathrm{~m}^{2}\end{array}$ & $1.6(1.4-1.9)$ & $6.1(5.7-6.6)$ & $27.3(20.2-42.3)$ & 0.2 & 16.9 \\
\hline CKD-EPI creatinine & 16 & $\begin{array}{l}73.8 \mathrm{~mL} / \\
\mathrm{min} / 1.73 \mathrm{~m}^{2}\end{array}$ & $1.2(1.0-1.4)$ & $5.3(5.1-5.6)$ & $24.3(18.0-37.7)$ & 0.2 & 14 \\
\hline CKD-EPI cystatin C & 17 & $\begin{array}{l}76.7 \mathrm{~mL} / \\
\mathrm{min} / 1.73 \mathrm{~m}^{2}\end{array}$ & $2.0(1.8-2.3)$ & $5.5(5.2-5.9)$ & $21.2(15.8-32.3)$ & 0.3 & 15.3 \\
\hline CKD-EPI creatinine-cystatin C & 16 & $\begin{array}{l}75.2 \mathrm{~mL} / \\
\mathrm{min} / 1.73 \mathrm{~m}^{2}\end{array}$ & $1.2(1.1-1.4)$ & $4.6(4.3-5.0)$ & $20.3(16.5-34.5)$ & 0.2 & 13.2 \\
\hline
\end{tabular}

\section{Subjects with CKD}

\begin{tabular}{|c|c|c|c|c|c|c|c|}
\hline Creatinine & 19 & $3.3 \mathrm{mg} / \mathrm{dL}$ & $1.3(1.1-1.5)$ & $2.5(2.4-2.7)$ & $28.1(21.2-41.6)$ & 0.1 & 7.9 \\
\hline Cystatin C & 18 & $2.7 \mathrm{mg} / \mathrm{L}$ & $0.8(0.7-1.0)$ & $3.2(3.0-3.4)$ & $27.2(20.4-40.8)$ & 0.1 & 9.1 \\
\hline MDRD & 19 & $\begin{array}{l}20.2 \mathrm{~mL} / \\
\mathrm{min} / 1.73 \mathrm{~m}^{2}\end{array}$ & $2.4(2.1-2.8)$ & $5.5(5.2-5.9)$ & $28.4(21.5-42.0)$ & 0.2 & 13.3 \\
\hline CKD-EPI ${ }_{\text {creatinine }}$ & 19 & $\begin{array}{l}20.1 \mathrm{~mL} / \\
\mathrm{min} / 1.73 \mathrm{~m}^{2}\end{array}$ & $2.6(2.3-3.0)$ & $5.2(4.9-5.6)$ & $30.0(22.7-44.4)$ & 0.2 & 14.4 \\
\hline CKD-EPI ${ }_{\text {cystatin C }}$ & 18 & $\begin{array}{l}23.8 \mathrm{~mL} / \\
\mathrm{min} / 1.73 \mathrm{~m}^{2}\end{array}$ & $1.2(1.1-1.4)$ & $7.3(6.8-7.8)$ & $38.7(29.1-58.1)$ & 0.2 & 20.1 \\
\hline CKD-EPI creatinine-cystatin C & 17 & $\begin{array}{l}20.9 \mathrm{~mL} / \\
\mathrm{min} / 1.73 \mathrm{~m}^{2}\end{array}$ & $1.6(1.4-1.8)$ & $5.4(5.1-5.8)$ & $35.2(26.4-52.9)$ & 0.2 & 15.1 \\
\hline
\end{tabular}

Except where stated otherwise, the data are percentage (\%) values. 95\% Confidence Intervals (CI) are shown between brackets

$\mathrm{CV}_{\mathrm{A}^{\prime}}$ analytical variation, $\mathrm{CV}_{\mathrm{I}}$, within-subject biological variation, $\mathrm{CV}_{\mathrm{G}^{\prime}}$, between-subject variation, II, index of individuality, RCV, reference change value

\section{$24 h$ variability profiles}

Figures 5.1 and 5.2 show $24 \mathrm{~h}$ variability profiles of creatinine, cystatin C, and all eGFR equations for both study groups. The mean creatinine and cystatin $C$ concentrations were by definition significantly lower in people without CKD than in those with CKD (creatinine: $1.0 \pm 0.3 \mathrm{mg} / \mathrm{dL}$ vs. $3.3 \pm 1.0 \mathrm{mg} / \mathrm{dL}, \mathrm{P}<0.01$; cystatin C: $1.0 \pm 0.3 \mathrm{mg} / \mathrm{L}$ vs. $2.7 \pm 0.8 \mathrm{mg} / \mathrm{L}, \mathrm{P}<0.01)$. Consequently, all average eGFR values were significantly higher in people without CKD, as compared to the people with CKD (MDRD: $75 \pm 21 \mathrm{~mL} / \mathrm{min} / 1.73 \mathrm{~m} 2$ vs. $20 \pm 9 \mathrm{~mL} / \mathrm{min} / 1.73 \mathrm{~m} 2 \mathrm{P}<0.01$; CKD-EPI creatinine: $74 \pm 18 \mathrm{~mL} / \mathrm{min} / 1.73 \mathrm{~m} 2$ vs. $20 \pm 10 \mathrm{~mL} / \mathrm{min} / 1.73 \mathrm{~m} 2, \mathrm{P}<0.01$; CKD-EPI 
cystatin C; $77 \pm 17 \mathrm{~mL} / \mathrm{min} / 1.73 \mathrm{~m} 2$ vs. $24 \pm 14 \mathrm{~mL} / \mathrm{min} / 1.73 \mathrm{~m} 2, \mathrm{P}<0.01$; CKD-EPI creatinine-cystatin C: $76 \pm 17 \mathrm{~mL} / \mathrm{min} / 1.73 \mathrm{~m} 2 \mathrm{vs} .21 \pm 12 \mathrm{~mL} / \mathrm{min} / 1.73 \mathrm{~m} 2, \mathrm{P}<0.01)$.

Creatinine concentrations increased up to $50 \%(0.46 \mathrm{mg} / \mathrm{dL})$ in people without CKD after dinner (participant 11, individual 24h profile of this participant was not shown). The increase was numerically similar in people with CKD $(0.40 \mathrm{mg} / \mathrm{dL})$ compared to people without CKD, but as a percentage the increase was substantially lower $(18 \%)$ due to the high baseline concentrations of creatinine in the people with CKD.

Unlike creatinine, cystatin C concentrations were not affected by dinner. Interestingly however, cystatin $C$ showed a small, but clearly evident diurnal rhythm, with decreasing cystatin c concentrations during daytime and increasing concentrations during the evening and night. This circadian rhythm of cystatin C was less prominent in people with CKD (Figures 5.1 and 5.2). In Supplemental Figure 5.2, the diurnal rhythm of cystatin $C$ was visualized by fitting a cosinor model through the average data points. For people without CKD the amplitude of the diurnal cosinor rhythm was $4.9 \pm 0.8$, whereas the amplitude was reduced to $1.6 \pm 0.5 \%$ for people with CKD.

Components of variation in people with and without CKD

Variation components of all parameters in both study groups are shown in table 5.2. $\mathrm{CV}_{\mathrm{G}}$ was comparable for the study groups. In both groups, $\mathrm{CV}_{\mathrm{G}}$ was significantly larger than $\mathrm{CV}_{\mathrm{I}}$ for all parameters. Due to the relatively high $\mathrm{CV}_{\mathrm{G}}$ values, all indexes of individuality were low (0.1 - 0.3). 
Figure 5.1. 24h variation profiles in subjects without CKD
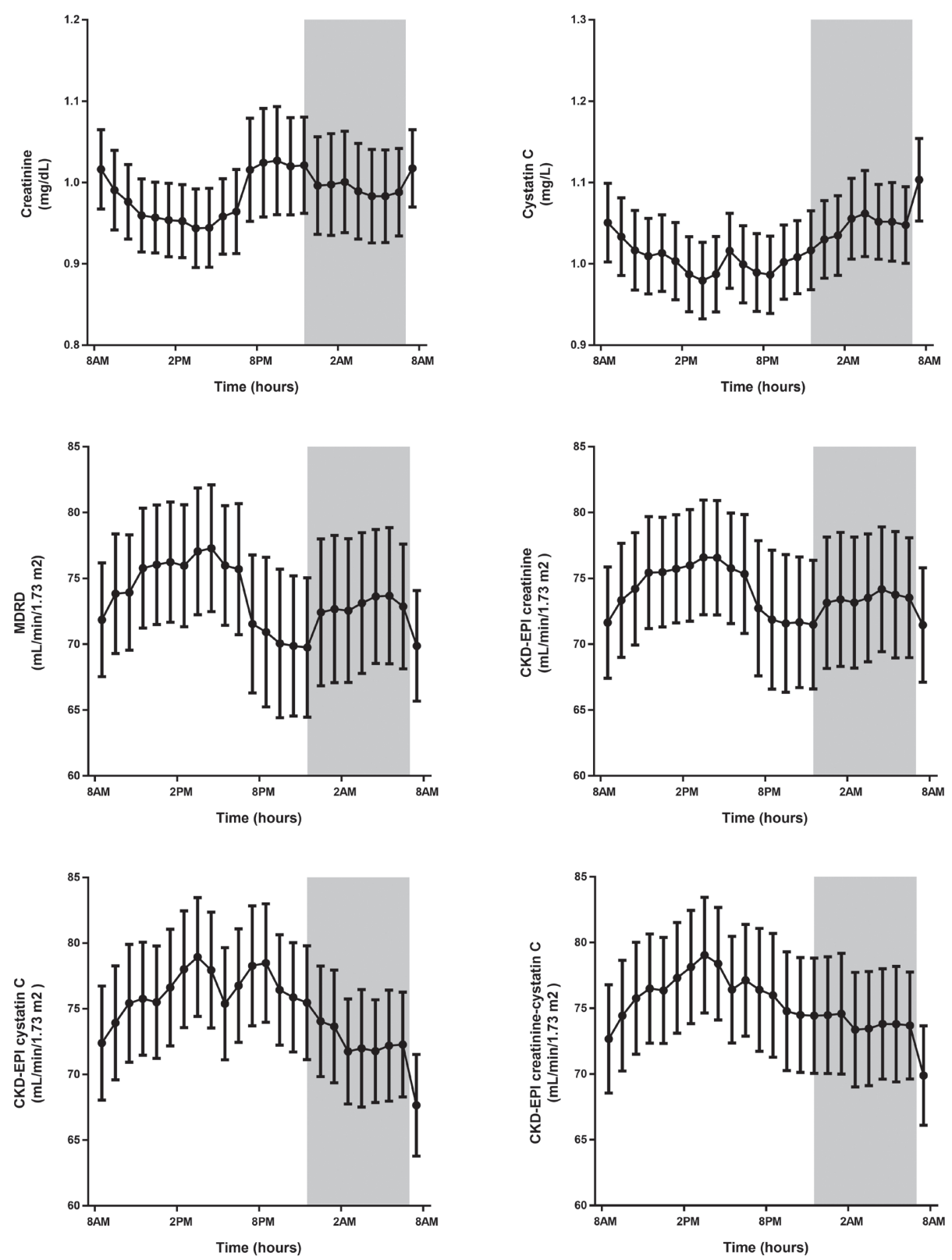

Values represent means \pm SEM. Subjects slept between 11:30 PM and 7 AM (shaded area). CKD, chronic kidney disease. SEM, standard error of the mean 
Figure 5.2. 24h variation profiles in subjects with CKD
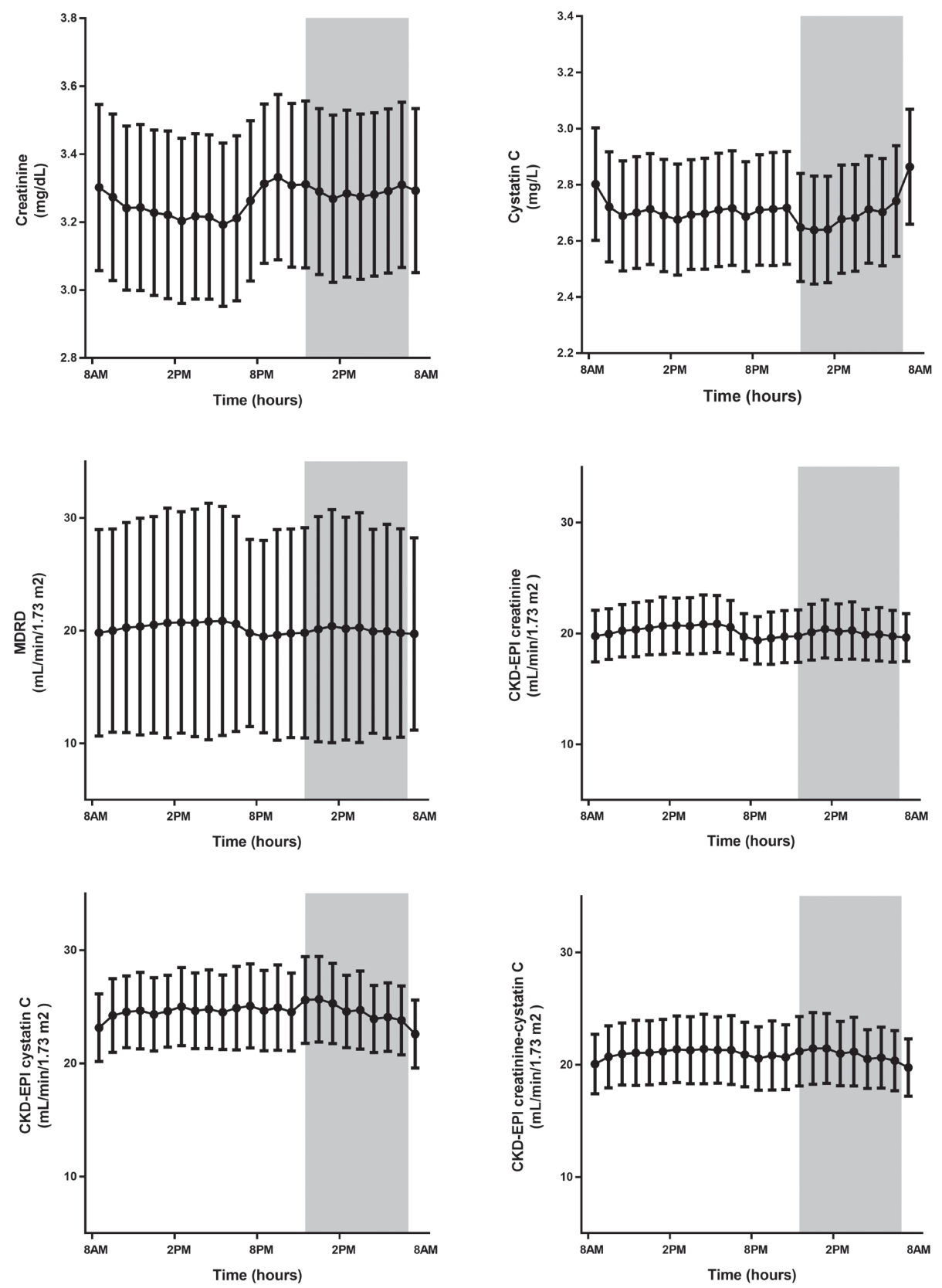

Values represent means \pm SEM. Subjects slept between 11:30 PM and 7 AM (shaded area). CKD, chronic kidney disease. SEM, standard error of the mean 
In Figure 5.3, the min-max concentration ranges of creatinine and cystatin $C$ are presented for the people without and with CKD. The people without CKD (panel A) exhibit a higher relative range in creatinine concentrations than the people with CKD (panel C). This difference in concentrations contributes to a significantly higher $\mathrm{CV}_{\mathrm{I}}$ expressed as percent in people without CKD (6.4\% vs. $\left.2.5 \%\right)$, and also the RCV was significantly higher in people without CKD (18\% vs. $8 \%$ ). The CV values of cystatin C, CKD-EPI cystatin C and CKD-EPI creatinine - cystatin C were also significantly different between the groups. However, these differences were smaller than for creatinine.

In people without CKD, the CKD-EPI based on the combination of creatinine and cystatin C (CKD-EPI creatinine-cystatin C) was the eGFR equation with lowest $C_{\mathrm{I}}$. In people with CKD, the CKD-EPI based on creatinine (CKD-EPI creatinine) demonstrated the lowest $\mathrm{CV}_{\mathrm{I}}$ over the day. However, in this group the difference with CKD-EPI creatinine - cystatin C was minimal (5.2\% vs. 5.4\%, with overlapping confidence intervals).

$\mathrm{CV}_{\mathrm{A}}$ was low in both study groups (maximum $2.0 \%$ for people without CKD and $2.6 \%$ for people with CKD). All $\mathrm{CV}_{\mathrm{A}}$ values met the 'desirable ratio' between $\mathrm{CV}_{\mathrm{I}}$ and $\mathrm{CV}_{\mathrm{A}^{\prime}}$ which is 1:2. A ratio below 0.5 assures that the analytical 'noise' contributes less than $12 \%$ to the total variation (7).

\section{Sensitivity analysis}

According to the Cochran within-subject outlier test, the 'after-dinner increase' of creatinine demanded the exclusion of some individuals (participants 14 and 30, respectively) (Supplemental Table 5.1 and Supplemental Figure 5.3. However, this strict statistical perspective may neglect the fact that the creatinine rises are in fact 'physiological changes', which also occur in hospitalized patients. We believe that excluding these participants may be too stringent and can lead to an underestimation of biological variation data. A sensitivity analysis included individuals that were excluded in the primary analysis due to heterogeneity of variance. In this analysis, slightly larger $\mathrm{CV}_{\mathrm{I}}$ values were revealed for most biomarkers (Supplemental Table 5.2). The difference was more pronounced for creatinine than for cystatin C (which lacks the after dinner rise), and the effect was stronger in people without CKD than in people with CKD. 


\section{Discussion}

In the current study, we present $24 \mathrm{~h}$ variation profiles of creatinine, cystatin $\mathrm{C}$, and their derived estimates of glomerular filtration rate (MDRD, CKD-EPI creatinine, CKD-EPI cystatin C, and CKD-EPI creatinine-cystatin C) in people without CKD, and in people with CKD. In addition, separate variation components were calculated and compared between both study groups.

An important finding of this study is that the $\mathrm{CV}_{\mathrm{I}}$ of creatinine is significantly higher in people without CKD than in people with CKD. In our study, the effect of the post-dinner creatinine spike on $\mathrm{CV}_{\mathrm{I}}$ was substantial especially for people without CKD, given that these people have low baseline creatinine concentrations (mean $1.0 \mathrm{mg} / \mathrm{dL}$ ). For people with $\mathrm{CKD}$, the effect on $\mathrm{CV}_{\mathrm{I}}{ }^{\prime} \mathrm{s}$ due to higher baseline creatinine concentrations (mean $3.3 \mathrm{mg} / \mathrm{dL}$ ) was smaller. This difference was even more pronounced in the sensitivity analysis that included the individuals with a (physiological) high post-dinner creatinine increase, likely due to meat consumption, increasing their $\mathrm{CV}_{\mathrm{I}}$ 's and marking them for removal based on statistical criteria. However, the variation in creatinine concentrations caused by dinner content is of interest for clinical practice, given that patients often have dinner with unknown meat and other content.

For clinical practice, this variation might ideally be included, even if this variation is statistically large and seemingly divergent. Rivara et al. performed a similar sensitivity analysis in which they showed a slight increase of the $\mathrm{CV}_{\mathrm{I}}$ of creatinine after including the individuals that were excluded in the primary analysis due to heterogeneity of variances (19).

The $\mathrm{CV}_{\mathrm{I}}$ for creatinine in this study was higher than reported in the EUBIVAS project (6.2\% vs. $4.4 \%$ ), which can likely be attributed to the fact that the creatinine concentrations measured in the EUBIVAS project were between-day $\mathrm{CV}_{\mathrm{I}}$ values based on fasting blood samples collected once per day at the same hour between 08.00 and 10.00 (20) (32). Therefore, creatinine increase after meat consumption is not integrated in the EUBIVAS $\mathrm{CV}_{\mathrm{I}}$ value. Since within-day $\mathrm{CV}_{\mathrm{I}}$ and between-day $\mathrm{CV}_{\mathrm{I}}$ are different concepts, the results of this study are not directly comparable to those of the EUBIVAS project.

The design of this study complies with the current checklists regarding biological variation as much as possible $(24,25)$. Only on item 7 of the most recent biological 
variation checklist, which is about the steady state condition do we deviate from the checklist by not including meat consumption in the model for the concentration of creatinine to create a 'steady state'. Whether or not meat consumption is included in a model for the concentration of creatinine, has consequences for the amount of variation left unexplained, and with that the size of the $\mathrm{CV}_{\mathrm{I}}$.

In a clinical setting, neither the time of dinner of a patient, nor the meat content of the dinner (or other meal), are known to the physician interpreting the creatinine laboratory results.

Figure 5.3. Min-max concentration ranges of creatinine and cystatin $C$ in subjects without and with CKD

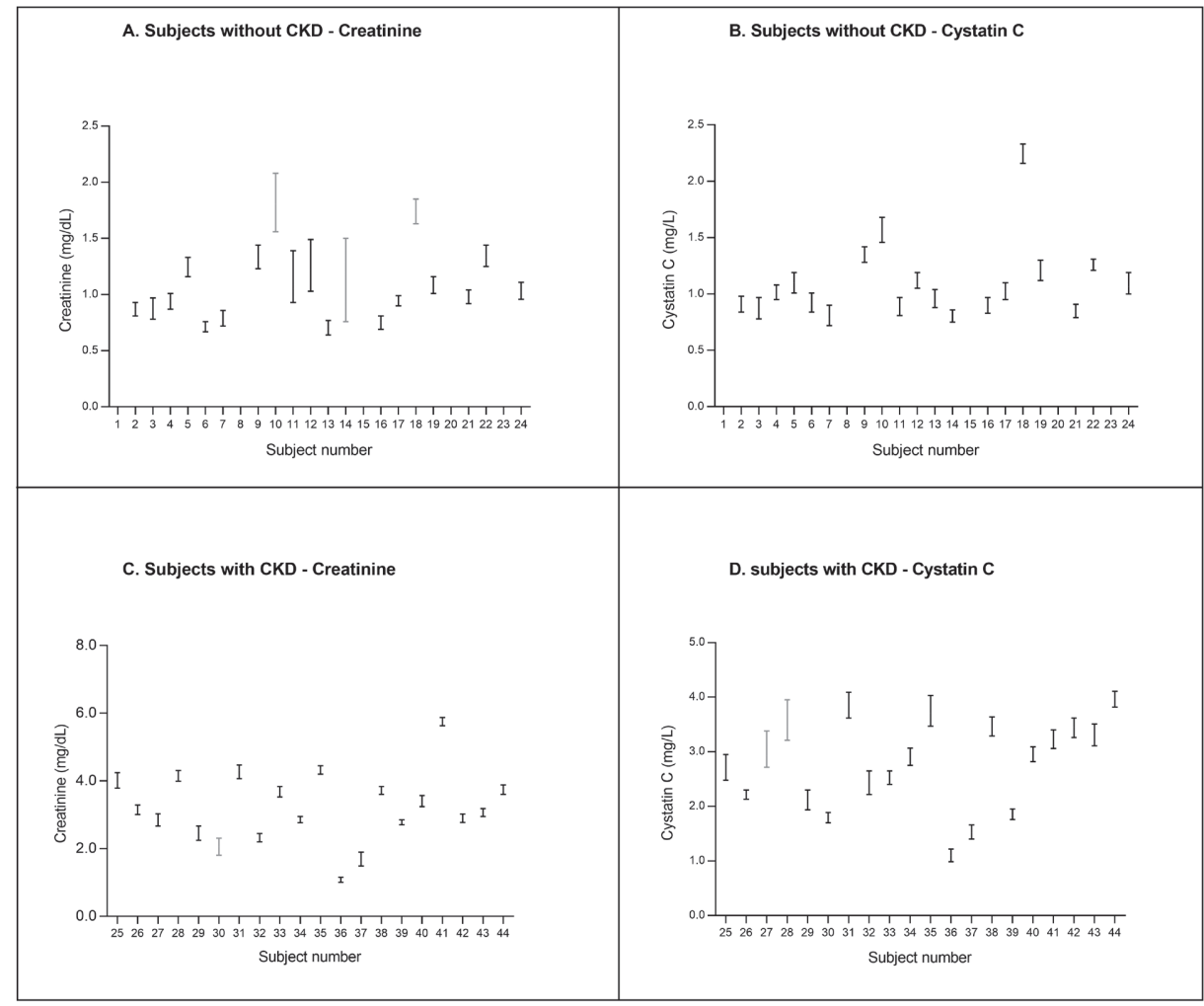

Panel A. Creatinine concentration ranges over $24 \mathrm{~h}$, subjects without CKD Panel B. Cystatin C concentration ranges over $24 \mathrm{~h}$, subjects without CKD Panel C. Creatinine concentration ranges over $24 \mathrm{~h}$, subjects with CKD Panel D. Cystatin C concentration ranges over $24 \mathrm{~h}$, subjects with CKD

Subjects in grey were excluded according to the outlier analyses CKD, chronic kidney disease 
Hence for clinical purposes, variation resulting from meat consumption should not be excluded from the model and should be included in the $\mathrm{CV}_{\mathrm{I}}$ estimate to allow for this additional uncertainty. Since most people consume meat in their diet, our $\mathrm{CV}_{\mathrm{I}}$ estimate for a 24 hour period can be considered to reflect typical physiologic conditions facing interpretation of creatinine and eGFR results.

Since this study was not originally designed to investigate the influence of meat consumption on creatinine concentrations, no detailed individual data of the participants regarding their meat consumption during the day were available. However, the study of Nair et al. standardized the meat consumption of all participants and compared their results with a non-meat meal. The Nair study described a significant increase of creatinine concentration after a meat meal for all study participants (33). For healthy volunteers and individuals with CKD stage 1 or 2 , the study reported statistically significant creatinine increases of $5 \mu \mathrm{mol} / \mathrm{L}(0.06$ $\mathrm{mg} / \mathrm{dL})$ and $8 \mu \mathrm{mol} / \mathrm{L}(0.09 \mathrm{mg} / \mathrm{dL})$, respectively. We observed a similar average increase of $0.07 \mathrm{mg} / \mathrm{dL}(6.2 \mu \mathrm{mol} / \mathrm{L})$ between 6PM-10PM presumably related to dinner composition. For people with CKD, the Nair study reported maximum creatinine increases of up to $0.25 \mathrm{mg} / \mathrm{dL}(22 \mu \mathrm{mol} / \mathrm{L})$, which is more pronounced than the average increase of $0.13 \mathrm{mg} / \mathrm{dL}(12 \mu \mathrm{mol} / \mathrm{L})$ we observed in the people with CKD.

Nevertheless, the study of Nair et al. emphasizes the finding that the relative fall in eGFR after meat consumption is proportionately less in patients with more advanced CKD stages (33), which is consistent with our findings. Another study by Preiss et al. demonstrated that the effect of meat consumption could even have an impact on diagnosis, due to misclassification of CKD staging if measurements are made after consuming a cooked-meat meal (34). The authors of this study state that physicians should ensure that, when classifying the stage of CKD, samples are taken under appropriate conditions (34). We endorse this conclusion, especially in the context of a 24-hour biological variation study. Creatinine was included in our study in order to be able to determine the calculated eGFR's and not to be an indicator for GFR (35).

Unlike creatinine, the $\mathrm{CV}_{\mathrm{I}}$ of cystatin $\mathrm{C}$ was in the same range for both people with or without CKD, and its $24 \mathrm{~h}$ profile was not characterized by a post-dinner spike. Despite substantial differences in biological variation and RCVs between cystatin C and creatinine, especially in people without CKD, these differences do 
not translate to similar differences in the biological variation and RCV's of the eGFR equations that are derived from these biomarkers. In fact, $\mathrm{CV}_{\mathrm{I}}$ values and RCVs of the CKD-EPI creatinine equation are of the same magnitude in both study groups. However, $\mathrm{CV}_{\mathrm{I}}$ values and RCV of the CKD-EPI cystatin $\mathrm{C}$ are of different magnitude. The transformation from cystatin C to CKD-EPI cystatin C leads to more dispersed CKD-EPI values than the transformation from creatinine to CKD-EPI creatinine.

Diurnal cystatin C profiles showed modest intrinsic diurnal rhythmicity, with a slight decrease during daytime, and increase during the evening and night. This is in line with modestly reduced glomerular filtration at nighttime compared to daytime $(36,37)$. A similar diurnal rhythmicity may be apparent in the creatinine profiles (at least a declining pattern from morning until evening), but is obscured by the post dinner-creatinine peak. Interestingly, the diurnal cystatin $C$ rhythm is somewhat diminished in people with CKD. This effect can be explained by reduced renal clearance in people with CKD, leading to accumulation of cystatin $C$, and consequent reduction in its diurnal rhythm (38).

Large rhythmic diurnal oscillations preclude calculation of overall variation components and RCVs, as they would become time-of day- dependent. Instead, hour-to-hour RCVs can be calculated, which take into account the structural change according to the diurnal rhythm. Such approach has been previously applied by our group and others for the calculation of RCVs for various hematological biomarkers and cardiac troponin $\mathrm{T}(39,40)$. However, rhythmic variation of cystatin $\mathrm{C}$ in this study was so small that the calculation of hour-to-hour variation components and RCVs would have complicated their interpretation without offering substantial benefit in terms of mathematical accuracy.

Some limitations of this study merit consideration: First, the design of the study was not limited to the assessment of the biological variation of kidney biomarkers. Although mealtimes were standardized across study participants, the content of the meals were not. Hence, the consumption of meat among meals was variable, and may have accounted for the highly variable creatinine rises, especially after dinner.

Second, the ratio between men and women was skewed $(77 \%$ men and $23 \%$ women), precluding robust assessment of potential gender differences. Third, the generalizability of this study to other ethnicities may be limited due to the fact that 
all participants were Caucasian. Fourth, most individuals, including those without CKD, had comorbidities. Nevertheless, the presence of comorbidities, and also the fact that the meals were not standardized, is representative for the average patient (both hospitalized and outpatient) and provides 'real world' estimates of the biological variation of interest when interpreting results.

In conclusion, we show that the $\mathrm{CV}_{\mathrm{I}}$ of creatinine is higher in people without CKD than in people with CKD. Despite differences in biological variation, RCVs of all derived estimates of glomerular filtration rate (MDRD, CKD-EPI creatinine, CKD-EPI cystatin C, and CKD-EPI creatinine-cystatin C) are within the same range (13-20\%) and are similar for people with or without CKD. 


\section{References}

1. Stevens PE, Levin A, Kidney Disease: Improving Global Outcomes Chronic Kidney Disease Guideline Development Work Group M. Evaluation and management of chronic kidney disease: Synopsis of the kidney disease: Improving global outcomes 2012 clinical practice guideline. Ann Intern Med 2013;158:825-30.

2. Levey AS, Stevens LA, Coresh J. Conceptual model of CKD: Applications and implications. Am J Kidney Dis 2009;53:S4-16.

3. Levey AS, Bosch JP, Lewis JB, Greene T, Rogers N, Roth D. A more accurate method to estimate glomerular filtration rate from serum creatinine: A new prediction equation. Modification of Diet in Renal Disease Study Group. Ann Intern Med 1999;130:461-70.

4. Inker LA, Schmid CH, Tighiouart H, Eckfeldt JH, Feldman HI, Greene T, et al. Estimating glomerular filtration rate from serum creatinine and cystatin C. N Engl J Med 2012;367:20-9.

5. Levey AS, Stevens LA, Schmid CH, Zhang YL, Castro AF, 3rd, Feldman HI, et al. A new equation to estimate glomerular filtration rate. Ann Intern Med 2009;150:604-12.

6. Carter JL, Parker CT, Stevens PE, Eaglestone G, Knight S, Farmer CK, Lamb EJ. Biological variation of plasma and urinary markers of acute kidney injury in patients with chronic kidney disease. Clin Chem 2016;62:876-83.

7. Fraser CG. Biological variation: From principles to practice AACC Press, Washington DC 2001.

8. Fraser CG, Harris EK. Generation and application of data on biological variation in clinical chemistry. Crit Rev Clin Lab Sci 1989;27:409-37.

9. Ozturk OG, Paydas S, Balal M, Sahin G, Karacor ED, Ariyurek SY, Yaman A. Biological variations of some analytes in renal posttransplant patients: A different way to assess routine parameters. J Clin Lab Anal 2013;27:438-43.

10. Delanaye P, Cavalier E, Depas G, Chapelle JP, Krzesinski JM. New data on the intraindividual variation of cystatin C. Nephron Clinical practice 2008;108:c246-8.

11. Larsson A, Akerstedt T, Hansson LO, Axelsson J. Circadian variability of cystatin C, creatinine, and glomerular filtration rate (GFR) in healthy men during normal sleep and after an acute shift of sleep. Chronobiol Int 2008;25:1047-61.

12. Carobene A, Graziani MS, Lo Cascio C, Tretti L, Cremonese E, Yabarek T, et al. Age dependence of within-subject biological variation of nine common clinical chemistry analytes. Clin Chem Lab Med 2012;50:841-4.

13. Reinhard M, Erlandsen EJ, Randers E. Biological variation of cystatin C and creatinine. Scand J Clin Lab Invest 2009;69:831-6.

14. Fraser CG, Williams P. Short-term biological variation of plasma analytes in renal disease. Clin Chem 1983;29:508-10.

15. Selvin E, Juraschek SP, Eckfeldt J, Levey AS, Inker LA, Coresh J. Within-person variability in kidney measures. Am J Kidney Dis 2013;61:716-22.

16. Pascoe PJ, Gallagher CS, Fraser CG. Components of biological variation of some serum analytes in hospitalized pregnant women. Clin Chem 1984;30:583-4.

17. Gowans EM, Fraser CG. Biological variation of serum and urine creatinine and creatinine clearance: Ramifications for interpretation of results and patient care. Ann Clin Biochem 1988;25 ( Pt 3):259-63.

18. Ravn B, Larsson A, Martensson J, Martling CR, Bell M. Intra-day variability of cystatin C, creatinine and estimated GFR in intensive care patients. Clin Chim Acta 2016;460:1-4.

19. Rivara MB, Zelnick LR, Hoofnagle AN, Newitt R, Tracy RP, Kratz M, et al. Diurnal and long-term variation in plasma concentrations and renal clearances of circulating markers of kidney proximal tubular secretion. Clin Chem 2017;63:915-23. 
20. Carobene A, Marino I, Coskun A, Serteser M, Unsal I, Guerra E, et al. The EuBIVAS project: Within-and between-subject biological variation data for serum creatinine using enzymatic and alkaline picrate methods and implications for monitoring. Clin Chem 2017;63:1527-36.

21. Klinkenberg LJ, Wildi K, van der Linden N, Kouw IW, Niens M, Twerenbold R, et al. Diurnal rhythm of cardiac troponin: Consequences for the diagnosis of acute myocardial infarction. Clin Chem 2016;62:1602-11.

22. van der Linden N, Hilderink JM, Cornelis T, Kimenai DM, Klinkenberg LJJ, van Doorn WP, et al. Twenty-four-hour biological variation profiles of cardiac troponin in individuals with or without chronic kidney disease. Clin Chem 2017;63:1655-6.

23. Association WM. Declaration of helsinki: Ethical principles for medical research involving human subjects. JAMA 2013;310:2191-4.

24. Bartlett WA, Braga F, Carobene A, Coskun A, Prusa R, Fernandez-Calle P, et al. A checklist for critical appraisal of studies of biological variation. Clin Chem Lab Med 2015;53:879-85.

25. Aarsand AK, T RR, Fernandez-Calle P, Ricos C, Diaz-Garzon J, Jonker N, et al. The biological variation data critical appraisal checklist:A standard for evaluating studies on biological variation. Clin Chem 2017 Dec 8. as doi: 10.1373/clinchem.2017.281808. [Epub ahead of print]

26. Roraas T, Petersen PH, Sandberg S. Confidence intervals and power calculations for withinperson biological variation: Effect of analytical imprecision, number of replicates, number of samples, and number of individuals. Clin Chem 2012;58:1306-13.

27. de Leur K, Vroemen JP, Vos DI, Elmans L, van der Laan L. Outcome after osteosynthesis of hip fractures in nonagenarians. Clin Interv Aging 2014;9:41-9.

28. Reed AH, Henry RJ, Mason WB. Influence of statistical method used on the resulting estimate of normal range. Clin Chem 1971;17:275-84.

29. Dixon WJ. Processing data for outliers. Biometrics 1953;9:74-89.

30. Burdick RK GF. Confidence intervals on variance components. New York, NY: Marcel Dekker 1992.

31. McDonald JH. Handbook of biological statistics reB-m, Maryland: Sparky House Publishing, 2014.

32. Carobene A, Strollo M, Jonker N, Barla G, Bartlett WA, Sandberg S, et al. Sample collections from healthy volunteers for biological variation estimates' update: A new project undertaken by the working group on biological variation established by the European Federation of Clinical Chemistry and Laboratory Medicine. Clin Chem Lab Med 2016;54:1599-608.

33. Nair S, O'Brien SV, Hayden K, Pandya B, Lisboa PJ, Hardy KJ, Wilding JP. Effect of a cooked meat meal on serum creatinine and estimated glomerular filtration rate in diabetes-related kidney disease. Diabetes Care 2014;37:483-7.

34. Preiss DJ, Godber IM, Lamb EJ, Dalton RN, Gunn IR. The influence of a cooked-meat meal on estimated glomerular filtration rate. Ann Clin Biochem 2007;44:35-42.

35. Diskin CJ. Creatinine and glomerular filtration rate: Evolution of an accommodation. Ann Clin Biochem 2007;44:16-9.

36. Wuerzner G, Firsov D, Bonny O. Circadian glomerular function: From physiology to molecular and therapeutical aspects. Nephrol Dial Transplant 2014;29:1475-80.

37. Koopman MG, Koomen GC, Krediet RT, de Moor EA, Hoek FJ, Arisz L. Circadian rhythm of glomerular filtration rate in normal individuals. Clin Sci (Lond) 1989;77:105-11.

38. van der Linden N, Cornelis T, Kimenai DM, Klinkenberg LJJ, Hilderink JM, Luck S, et al. Origin of cardiac troponin T elevations in chronic kidney disease. Circulation 2017;136:1073-5.

39. Hilderink JM, Klinkenberg LJJ, Aakre KM, de Wit NCJ, Henskens YMC, van der Linden N, et al. Within-day biological variation and hour-to-hour reference change values for hematological parameters. Clin Chem Lab Med 2017;55:1013-24.

40. Aakre KM, Roraas T, Petersen PH, Svarstad E, Sellevoll H, Skadberg O, et al. Weekly and 90minute biological variations in cardiac troponin $\mathrm{T}$ and cardiac troponin I in hemodialysis patients and healthy controls. Clin Chem 2014;60:838-47. 


\section{Supplemental material}

\section{Supplemental figure 5.1. Study flow diagram}

Individuals included without CKD

$(n=24)$

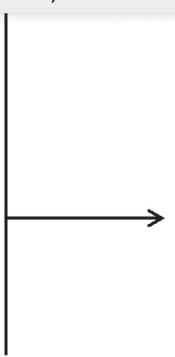

Individuals excluded in advance:

Participants 1,8, 15, and 23

(incomplete data)

Participant 20

(unstable situation)

Participants 10 and 18

(high creatinine values)

$n=19$

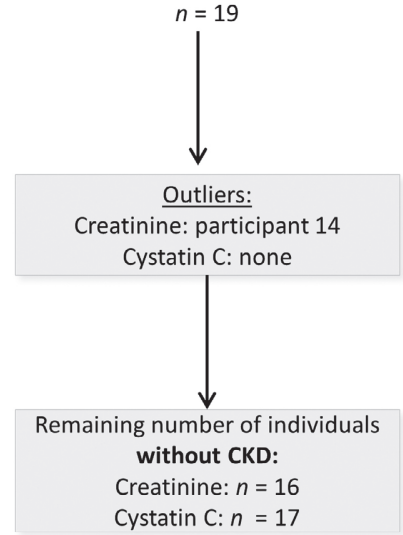

CKD, chronic kidney disease

Individuals included with CKD

( $n=20)$

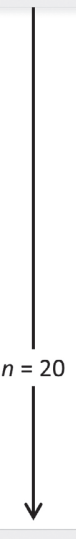

Outliers:

Creatinine: participant 30

Cystatin C: participants 27 and 28

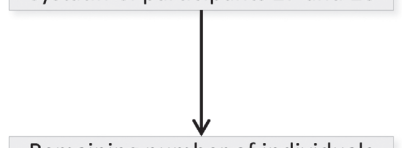

Remaining number of individuals with CKD:

Creatinine: $n=19$

Cystatin C: $n=18$ 
Supplemental table 5.1. Overview of outliers and excluded subjects per parameter

Excluded subjects Cochran's C test

Cochran's C test Reed's criterion in advance (analytical outliers)

(within-subject) (between-subject)

Subjects without CKD

Creatinine Subjects $1,8,10$, None $15,18,20$ and 23

Cystatin C Subjects 1, 8, 10,

$15,18,20$ and 23
.

Subject 13, None

Subject 14

None measurement 20

None None

Subjects with CKD

Creatinine None

None

Subject 30

None

Cystatin C None

None

Subjects 27 and 28 None

\section{Supplemental Figure 5.2. Diurnal rhythm of cystatin C}

A. Diurnal rhythm cystatin C - subjects without CKD

B. Diurnal rhythm cystatin C - subjects with CKD
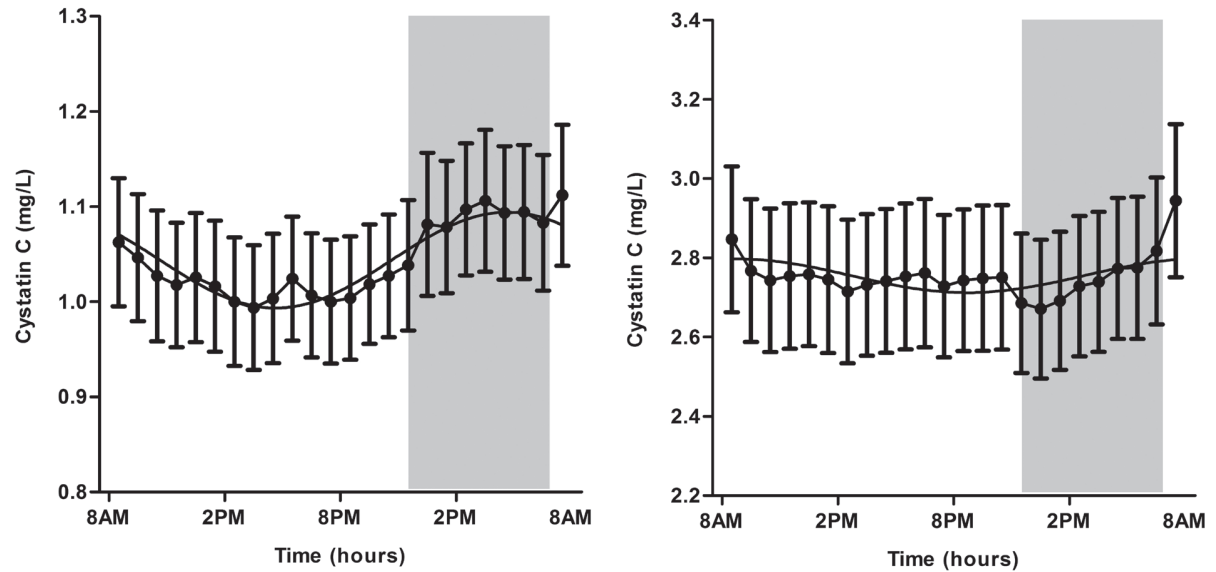

Panel A: Mean data points \pm SEM and cosine curve on the group level of the people without CKD. Amplitude $=4.9 \pm 0.8 \%\left(R^{2}=0.88\right)$. SEM, standard error of the mean Panel B: Mean data points \pm SEM and cosine curve on the group level for the people with CKD. Amplitude $=1.6 \pm 0.5 \%\left(R^{2}=0.32\right)$. SEM, standard error of the mean 
Supplemental Table 5.2. Sensitivity analysis: Components of biological variation of creatinine, cystatin C and eGFR for both study groups, including individuals excluded from primary analysis due to heterogeneity of variances in creatinine (subjects 14 and 30 )

$\begin{array}{lllllll}n & \text { Mean } & \mathrm{CV}_{\mathrm{A}} & \mathrm{CV}_{\mathrm{I}} & \mathrm{CV}_{\mathrm{G}} & \text { II } & \mathrm{RCV} \\ \end{array}$

\section{Subjects without CKD}

\begin{tabular}{|c|c|c|c|c|c|c|c|}
\hline Creatinine & 17 & $1.0 \mathrm{mg} / \mathrm{dL}$ & $1.1(1.0-1.3)$ & $8.7(8.2-9.4)$ & $20.4(15.2-31.2)$ & 0.4 & 24.4 \\
\hline Cystatin C & 17 & $1.0 \mathrm{mg} / \mathrm{dL}$ & $1.1(1.0-1.3)$ & $4.1(3.8-4.4)$ & $15.3(11.3-23.2)$ & 0.3 & 11.6 \\
\hline MDRD & 17 & $\begin{array}{l}75.3 \mathrm{~mL} / \\
\mathrm{min} / 1.73 \mathrm{~m}^{2}\end{array}$ & $1.6(1.4-1.9)$ & $8.6(8.0-9.2)$ & $26.4(19.7-40.3)$ & 0.3 & 23.8 \\
\hline CKD-EPI creatinine & 17 & $\begin{array}{l}73.8 \mathrm{~mL} / \\
\mathrm{min} / 1.73 \mathrm{~m}^{2}\end{array}$ & $1.2(1.0-1.4)$ & $7.6(7.1-8.2)$ & $23.5(17.5-35.9)$ & 0.3 & 21.1 \\
\hline CKD-EPI ${ }_{\text {cystatin C }}$ & 17 & $\begin{array}{l}76.7 \mathrm{~mL} / \\
\mathrm{min} / 1.73 \mathrm{~m}^{2}\end{array}$ & $2.0(1.8-2.3)$ & $5.5(5.2-5.9)$ & $21.2(15.8-32.3)$ & 0.3 & 15.3 \\
\hline CKD-EPI ${ }_{\text {creatinine-cystatin C }}$ & 17 & $\begin{array}{l}75.9 \mathrm{~mL} / \\
\mathrm{min} / 1.73 \mathrm{~m}^{2}\end{array}$ & $1.2(1.1-1.4)$ & $5.8(5.4-6.2)$ & $21.7(16.1-33.0)$ & 0.3 & 16.3 \\
\hline
\end{tabular}

\section{Subjects with CKD}

\begin{tabular}{|c|c|c|c|c|c|c|c|}
\hline Creatinine & 20 & $3.2 \mathrm{mg} / \mathrm{dL}$ & $1.3(1.1-1.5)$ & $2.8(2.6-2.9)$ & $28.2(21.4-41.2)$ & 0.1 & 8.4 \\
\hline Cystatin C & 18 & $2.7 \mathrm{mg} / \mathrm{L}$ & $0.8(0.7-1.0)$ & $3.5(3.3-3.8)$ & $25.1(19.1-36.7)$ & 0.1 & 10.1 \\
\hline MDRD & 20 & $\begin{array}{l}20.8 \mathrm{~mL} / \\
\mathrm{min} / 1.73 \mathrm{~m}^{2}\end{array}$ & $2.4(2.1-2.8)$ & $4.8(4.5-5.1)$ & $30.8(23.4-44.9)$ & 0.2 & 15.2 \\
\hline CKD-EPI creatinine & 20 & $\begin{array}{l}20.8 \mathrm{~mL} / \\
\mathrm{min} / 1.73 \mathrm{~m}^{2}\end{array}$ & $\begin{array}{l}2.6(2.3- \\
3.0)\end{array}$ & $5.9(5.5-6.3)$ & $32.2(24.5-47.0)$ & 0.2 & 16.3 \\
\hline CKD-EPI cystatin C & 18 & $\begin{array}{l}23.8 \mathrm{~mL} / \\
\mathrm{min} / 1.73 \mathrm{~m}^{2}\end{array}$ & $1.2(1.1-1.4)$ & $7.3(6.8-7.8)$ & $38.3(29.1-56.0)$ & 0.2 & 20.2 \\
\hline CKD-EPI ${ }_{\text {creatinine-cystatin C }}$ & 18 & $\begin{array}{l}21.6 \mathrm{~mL} / \\
\mathrm{min} / 1.73 \mathrm{~m}^{2}\end{array}$ & $1.6(1.4-1.8)$ & $5.4(5.1-5.8)$ & $34.3(26.1-50.2)$ & 0.2 & 15.0 \\
\hline
\end{tabular}

Except where stated otherwise, the data are percentage (\%) values. 95\% Confidence Intervals (CI) are shown between brackets. $\mathrm{CV}_{\mathrm{A}^{\prime}}$ analytical variation, $\mathrm{CV}_{\mathrm{I}^{\prime}}$, within-subject biological variation, $\mathrm{CV}_{\mathrm{G}^{\prime}}$ between-subject variation, II, index of individuality, RCV, reference change value 
Supplemental figure 5.3. Individual $24 \mathrm{~h}$ creatinine curves of 'outlier' subjects
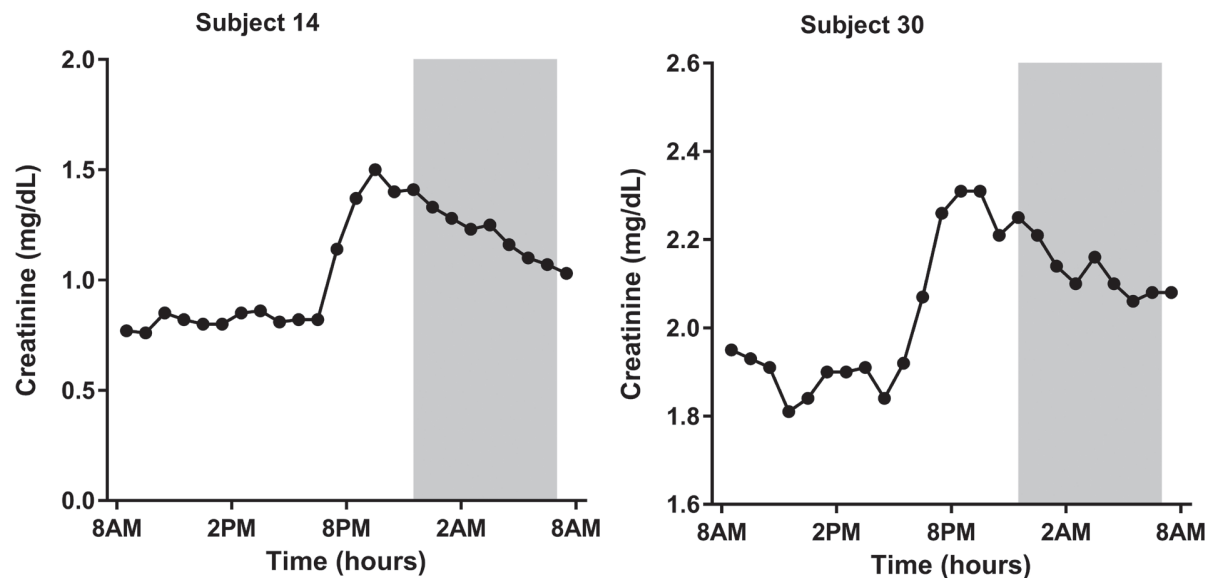

Values represent creatinine concentrations (mg/dL). Subjects slept between 11:30 PM and 7 AM (shaded area). 


\section{Chapter 6}




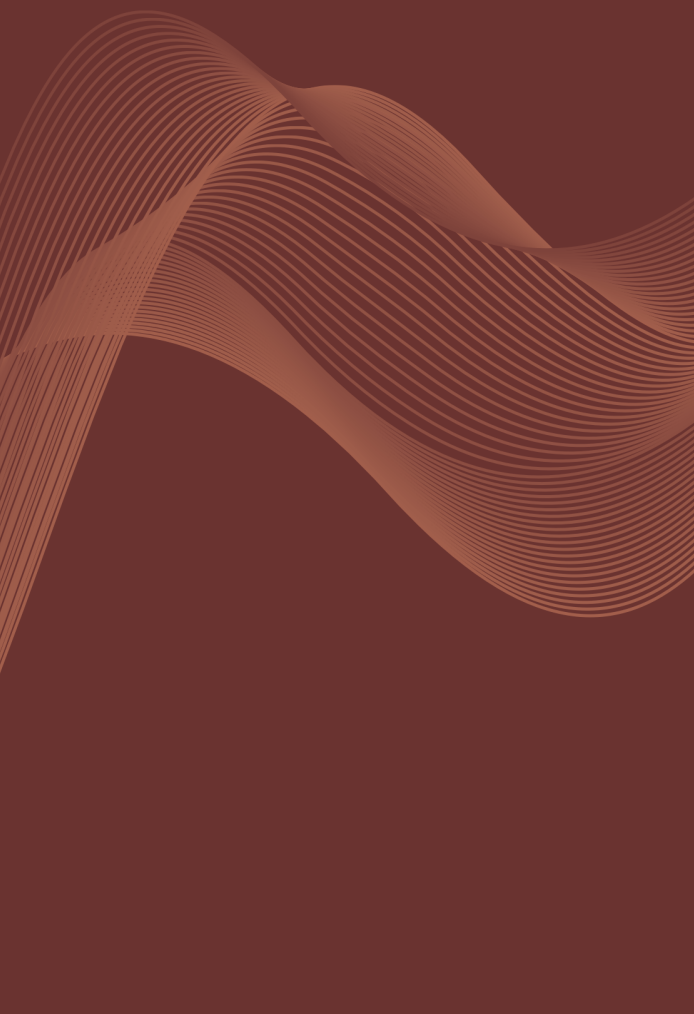

\section{Twenty-four hour biological variation profiles of cardiac troponin I in subjects with or without chronic kidney disease}

van der Linden N, Hilderink JM, Cornelis T, Kimenai DM, Klinkenberg LJJ, van Doorn WP, Litjens EJR, van Suijlen JDE, van Loon LJC, Bekers O, Kooman JP, Meex SJR 


\section{Abstract}

\section{Background}

Correct interpretation of serial cardiac troponin measurements is of profound importance for the diagnosis of acute myocardial infarction, especially in populations with a high prevalence of persistently elevated cardiac troponin levels. To gain more insight in physiological cardiac troponin I fluctuations, we performed a biological variation study in which we examined and compared the hour-to-hour biological variation in cardiac troponin I over 24-hours in subjects with and subjects without chronic kidney disease.

\section{Methods}

During 24h, hourly blood samples were collected from 20 subjects with and 24 subjects without chronic kidney disease. Cardiac troponin I was determined in duplicate. Analytical variation $\left(\mathrm{CV}_{\mathrm{A}}\right)$, within-subject biological variation $\left(\mathrm{CV}_{\mathrm{I}}\right)$ and between-subject biological variation $\left(\mathrm{CV}_{\mathrm{G}}\right)$ were calculated for 1-hour, 3-hour and 6-hour intervals in both groups.

\section{Results}

Baseline cardiac troponin I levels were higher in subjects with chronic kidney disease compared to those without (6.8 [3.5-9.2] ng/L vs. 4.7 [2.8-6.9] ng/L; $\mathrm{p}=0.09)$. The within-day variation showed no diurnal pattern. Variation components remained constant for all time-intervals. Whereas $\mathrm{CV}_{\mathrm{G}}(49-95 \%)$ and $\mathrm{CV}_{\mathrm{I}}(8-9 \%)$ were not significantly different between both groups, $\mathrm{CV}_{\mathrm{A}}$ was significantly lower in the group with the highest concentrations, the subjects with chronic kidney disease (6\% vs. $10 \%)$.

\section{Conclusions}

Cardiac troponin I concentrations fluctuate at random during the day. Except from the analytical variation, variation components were of the same magnitude in subjects with or without CKD. Our findings imply that the variation in cardiac troponin I levels is not affected by the presence of chronic kidney disease. 


\section{Background}

The interpretation of cardiac troponin concentrations at presentation and their dynamics over time is a key aspect in the diagnostic workup of acute myocardial infarction in the absence of characteristic ECG-abnormalities (1, 2). Whereas subjects with very low or high cardiac troponin concentrations at presentation can immediately be ruled-out or ruled-in respectively, serial measurements are particularly indicated in patients with moderately elevated cardiac troponin concentrations (3-6). A majority of these patients does not have an acute myocardial infarction, but shows persistently elevated cardiac troponin concentrations which are associated with the presence of underlying (subclinical) diseases like diabetes, coronary artery disease, hypertension and chronic kidney disease (CKD) (7-9). Especially in these patients it is of profound importance to distinguish pathology associated changes from physiological fluctuation in serial cardiac troponin measurements.

Biological variation studies provide insight in the magnitude of physiological variation. On the basis is the assumption that the biomarker concentrations fluctuate around an individual set point, and that the totally observed variation can be subdivided in a between-subject, a within-subject and an analytical component (10). These individual components facilitate the estimation of the reference change value (RCV), the required threshold for a change between serial measurements to be significantly different from a physiological variation. Additionally it is also possible to calculate the index of individuality (II), which represents the variation between the individual set points (11-13).

During the last decade, the duration of the diagnostic workup for acute myocardial infarction shortened, which was reflected in the recent introduction of the $0 \mathrm{~h} / 1 \mathrm{~h}$ algorithm $(1,14,15)$. Since most biological variation studies focus on long(er)-term variation in cardiac troponin I (cTnI) (16-19), less is known about the hour-to-hour variation. In addition, all previous studies were limited to a certain time interval and it is hence not clear whether the derived values apply to both day and night, or may be affected by a diurnal rhythm (20). The present biological variation study was the first to examine and compare the hour-to-hour biological variation in CTnI over 24-hours in subjects with and subjects without CKD. 


\section{Material and Methods}

This study was carried out according to the principles of the Declaration of Helsinki, approved by the Institutional Review Board and Ethics Committee of Maastricht University Medical Center, and registered at clinicaltrial.gov (NCT02091427 and NCT02210897). All participants provided written informed consent. The current study meets the critical appraisal checklist criteria for studies of biological variation as proposed by Bartlett et al. (21).

\section{Study population}

The study population consisted of 44 adult subjects, 24 subjects ( 19 males and 5 females) without CKD and 20 subjects ( 14 males and 6 females) with CKD. The subjects without CKD were previously described in more detail (20). Subjects with CKD stage 3 or higher (estimated glomerular filtration rate (eGFR) $<59 \mathrm{~mL}$ / $\min / 1.73 \mathrm{~m} 2)(22)$ were included at the nephrology outpatient department of the Maastricht University Medical Center (MUMC), and suffered from various types of kidney disease (glomerular $(n=7)$, tubulointerstitial $(n=4)$, vascular $(n=8)$ and congenital kidney disease $(n=1)$ ). Exclusion criteria were: history of acute myocardial infarction less than 12 months prior to exclusion, (current) dialysis regimen, active cardiac disease (angina pectoris, cardiomyopathy and myocarditis) and anemia $(\mathrm{Hb}<10.5 \mathrm{~g} / \mathrm{dL})$. To monitor whether the degree of renal impairment was stable in CKD subjects, we examined kidney function (eGFR) one week and one month after the initial test day.

\section{Study design}

After an overnight fast, subjects arrived at 8 a.m. at the laboratory by car of public transport. For $25 \mathrm{~h}$, from 8.30 A.M. till 9.30 A.M. the next day, subjects were restricted to the laboratory environment, and samples were collected every hour from an antecubital venous catheter. Extension lines for blood sampling were used to prevent disturbance of participants' sleep during the night. Meals were consumed at 8.30 A.M., 12.30 P.M. and 6.00 P.M. (breakfast, lunch and dinner, respectively). Subjects went to bed at 11.30 P.M., lights were off between 11.35 P.M. and 7.00 A.M., and they slept in a supine position. Participants were asked to refrain from exhaustive physical activities and exercise training, two days before the test day.

\section{Laboratory measurements}

Blood samples were collected in ethylenediaminetetraacetic acid containing (EDTA) tubes. Samples were centrifuged and the plasma was stored at $-80^{\circ} \mathrm{C}$ until analysis. 
cTnI was measured with the STAT high-sensitivity troponin I assay (Architect, Abbott Diagnostics). According to the manufacturer, the 99th percentile of this assay is $26.2 \mathrm{ng} / \mathrm{L}$ with a corresponding coefficient of variation of $<5 \%$. To enable the determination of analytical variation, all samples were measured in duplicate. Creatinine and cystatin C were both measured on the COBAS 6000 analyzer. The estimated glomerular filtration rate (eGFR) was calculated according to the Chronic Kidney Disease Epidemiology Collaboration (CKD-EPI) formula based on creatinine and cystatin C (23).

\section{Statistical analysis}

For the comparison between the baseline characteristics in both groups, continuous variables were compared with the Mann-Whitney $U$ test, and categorical variables were compared by use of the Pearson $\chi 2$ test.

For our 24-hour analyses, we used the data obtained between 10.30 AM (first day) till 9.30 (AM) (second day). As suggested by Fraser and Harris (24), we tested for homogeneity in the analytical- and within-subject biological variances using the method described by Cochran. Finally, the criteria of Reed were used to identify between-subject outliers level (10).

Two-fold nested ANOVA was used to calculate the coefficients of variation on a between-subject $\left(\mathrm{CV}_{\mathrm{G}}\right)$, within-subject $\left(\mathrm{CV}_{\mathrm{I}}\right)$ and an analytical $\left(\mathrm{CV}_{\mathrm{A}}\right)$ level, including $95 \%$ confidence intervals $(25,26)$. We calculated the reference change value (RCV) using the formula:

$$
\mathrm{RCV}=\mathrm{Z} * \sqrt{2 *\left(C V_{A}^{2}+C V_{I}^{2}\right.}
$$

In the RCV formula, Z represents the number of standard deviations appropriate for the desired level of statistical significance for a bidirectional change (10). For the RCV calculations in this study, a Z-score of 1.96 was used. The index of individuality (II) was calculated using the formula $(13,27)$ :

$$
\mathrm{II}=\frac{\left.\sqrt{(} C V_{I}^{2}+C V_{A}^{2}\right)}{C V_{G}}
$$

All hypothesis testing was two-tailed, and values of $\mathrm{P}<0.05$ were considered statistically significant. Statistical analyses were performed with SPSS for Windows 23.0 (IBM Corporation). 


\section{Results}

A total of 44 subjects, 20 with and 24 without CKD disease, participated in this biological variation study. We plotted the range in observed cTnI concentrations over $24 \mathrm{~h}$ for all individual subjects in Figure 6.1. Complete data sets were available for 40 subjects (subjects 1, 8, 15 and 23 had missing data). In addition, one subject had no measurable levels of cTnI (subject 36), and another subject became sick during the test day (subject 20). Two subjects (subjects 3 and 44) were identified as outliers and were therefore excluded from further analyses. A total of 36 subjects, 18 subjects with and 18 subjects without CKD, were included in the final data analyses.

Figure 6.1. Subject specific concentrations over $24 \mathrm{~h}$ with hourly sampling

A

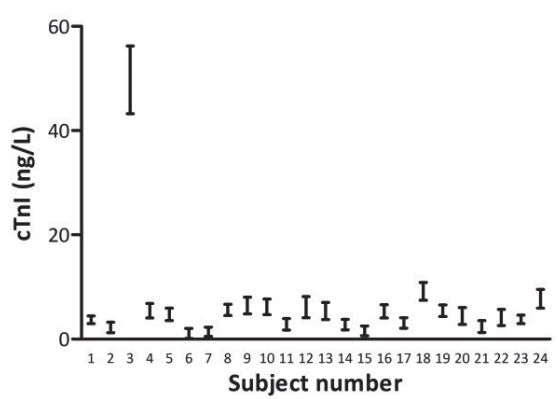

B

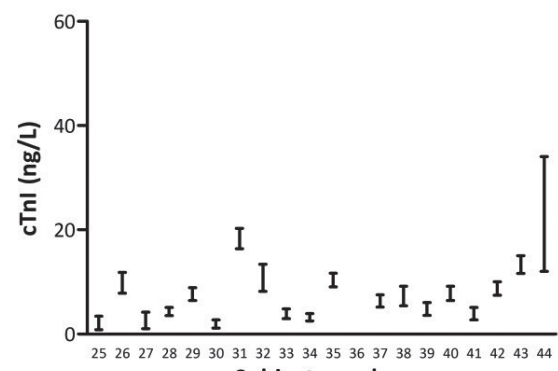

Absolute ranges (min - max) of CTnI concentrations over 24h in subjects without CKD (panel A) and with CKD (panel B).

Figure 6.2 shows the in- and exclusion of subjects, including baseline characteristics. In subjects with CKD the eGFR remained for at least one month after the initial test day (data not shown).

Baseline cTnI levels where higher in subjects with CKD compared to those without CKD (6.8 [3.5-9.2] ng/L vs. 4.7 [2.8-6.9] ng/L; $p=0.09)$. The within-day variation in these concentrations showed no diurnal pattern, and biological variation seemed to be constant over $24 \mathrm{~h}$ in both groups (Figure 6.3, Supplemental Figure 6.1) (individual curves of subjects without CKD have been previously published (20)). Consecutively, we calculated the variation components of 1-hour, 3-hour and 6-hour intervals during the day, which are presented in Table 6.1. We detected no significant differences in the components of variation for the different time intervals. When the variation components where compared between subjects without and subjects with $\mathrm{CKD}, \mathrm{CV}_{\mathrm{G}}$ and $\mathrm{CV}_{\mathrm{I}}$ were not significantly different. In 
contrast, $\mathrm{CV}_{\mathrm{A}}$ was significantly smaller in subjects with $\mathrm{CKD}$, the group with the higher CTnI concentrations. This lower $\mathrm{CV}_{\mathrm{A}}$ contributed to lower RCV's among these subjects. Besides, we observed a trend towards a higher between-subject variation and a lower index of individuality in this group.

Table 6.1. Hour-to-hour biological variation in subjects with or without chronic kidney disease

\begin{tabular}{|c|c|c|c|c|c|c|}
\hline & \multicolumn{2}{|c|}{ 1-hour } & \multicolumn{2}{|c|}{ 3-hourc } & \multicolumn{2}{|c|}{ 6-hourd } \\
\hline & $\begin{array}{c}\text { No CKD } \\
n=18\end{array}$ & $\begin{array}{l}\text { CKD } \\
n=18\end{array}$ & $\begin{array}{c}\text { No CKD } \\
n=18\end{array}$ & $\begin{array}{l}\text { CKD } \\
n=18\end{array}$ & $\begin{array}{c}\text { No CKD } \\
n=18\end{array}$ & $\begin{array}{l}\text { CKD } \\
n=18\end{array}$ \\
\hline \multicolumn{7}{|c|}{ Variance components } \\
\hline $\mathrm{CV}_{\mathrm{G}}$ & $\begin{array}{c}49.4 \\
(37.1-74.2)\end{array}$ & $\begin{array}{c}62.4 \\
(46.8-93.5)\end{array}$ & $\begin{array}{c}48.0 \\
(35.9-71.1)\end{array}$ & $\begin{array}{c}61.6 \\
(46.2-92.4)\end{array}$ & $\begin{array}{c}48.6 \\
(36.2-73.1)\end{array}$ & $\begin{array}{c}61.6 \\
(46.1-92.5)\end{array}$ \\
\hline$C V_{I}$ & $\begin{array}{c}8.6 \\
(7.6-9.7)\end{array}$ & $\begin{array}{c}7.7 \\
(7.1-8.4)\end{array}$ & $\begin{array}{c}9.4 \\
(7.6-11.5)\end{array}$ & $\begin{array}{c}8.7 \\
(7.5-10.2)\end{array}$ & $\begin{array}{c}9.2 \\
(6.2-12.4)\end{array}$ & $\begin{array}{c}8.9 \\
(7.1-11.3)\end{array}$ \\
\hline$C V_{A}{ }^{a}$ & $\begin{array}{c}10.0 \\
(9.4-10.8)\end{array}$ & $\begin{array}{c}5.6 \\
(5.2-6.0)\end{array}$ & $\begin{array}{c}9.9 \\
(8.8-11.2)\end{array}$ & $\begin{array}{c}5.6 \\
(5.0-6.3)\end{array}$ & $\begin{array}{c}10.1 \\
(8.7-12.1)\end{array}$ & $\begin{array}{c}5.9 \\
(5.0-7.0)\end{array}$ \\
\hline \multicolumn{7}{|l|}{$\mathbf{R C V}^{\mathrm{b}}$} \\
\hline Normal & 36.7 & 26.4 & 37.9 & 28.6 & 37.5 & 29.5 \\
\hline Log-normal & $44.0 ;-30.6$ & $30.18 ;-23.16$ & $45.8 ;-31.4$ & $33.0 ;-24.8$ & $45.7 ;-31.4$ & $34.2 ;-25.5$ \\
\hline $\begin{array}{l}\text { Index of } \\
\text { individuality }\end{array}$ & 0.27 & 0.15 & 0.28 & 0.17 & 0.28 & 0.17 \\
\hline
\end{tabular}

Values are \% $(95 \% \mathrm{CI}) ; \mathrm{CI}=$ confidence interval; $\mathrm{CV}_{\mathrm{A}}=$ analytical coefficient of variation; $\mathrm{CV}_{\mathrm{G}}=$ between-subject coefficient of variation; $\mathrm{CV}_{\mathrm{I}}=$ within-subject biological coefficient of variation; $\mathrm{RCV}=$ reference change value

${ }^{a}$ on the basis of duplicate measurements

b on the basis of a Z-score of 1.96

c 3-hour intervals: 10:30AM-01:30PM, 01:30PM-04:30PM, 04:30PM-07:30PM, 07:30PM-10:30PM, 01:30AM-4:30AM and 4:30AM-7:30AM

d 6-hour intervals: 10:30AM-04:30PM, 04:30PM-10:30PM and 10:30PM-04:30AM

\section{Discussion}

This study examined the hour-to-hour biological variation of cTnI, and calculated its variation components over 24-hours in subjects with or without CKD. We report four major findings: First, we observed randomly fluctuating CTnI levels over the day. In the absence of a circadian rhythm, we calculated the 1-hour, 3-hour and 6-hour variation over the day without any additional transformations. The fact that the $\mathrm{CV}_{\mathrm{I}}$ remained constant (8-9\%) during all time-intervals over 24-hours, supports 
our observation of random variation. Second, the $\mathrm{CV}_{\mathrm{I}}$ was not significantly different in subjects with or without CKD. This seems to be in line with the claim that for the majority of parameters $\mathrm{CV}_{\mathrm{I}}$ values are in the same order in health and disease (10, 28). Third, we identified a substantial amount of variation between individuals ( $C V_{G}$ $=48-62 \%)$. This finding corresponds to that of previous studies $(16-18,29,30)$, and, since the $\mathrm{CV}_{\mathrm{I}}$ is rather limited, it produces a low index of individuality $(<0.3)$. The observation that the $\mathrm{CV}_{\mathrm{G}}$ is slightly higher in subjects with CKD may be due to a high variety in comorbidities characteristic for this population (31). Fourth, we showed that the $\mathrm{CV}_{\mathrm{A}}$ is significantly higher in subjects without than in those with CKD (10\% vs. $6 \%$ ). This can be explained by the concentration-dependency of the $\mathrm{CV}_{\mathrm{A}}$. As a consequence of these differences in $\mathrm{CV}_{\mathrm{A}^{\prime}}$, the RCV is slightly lower in subjects with CKD.

Figure 6.2. Flowchart of the in- and exclusion of subjects, including baseline characteristics

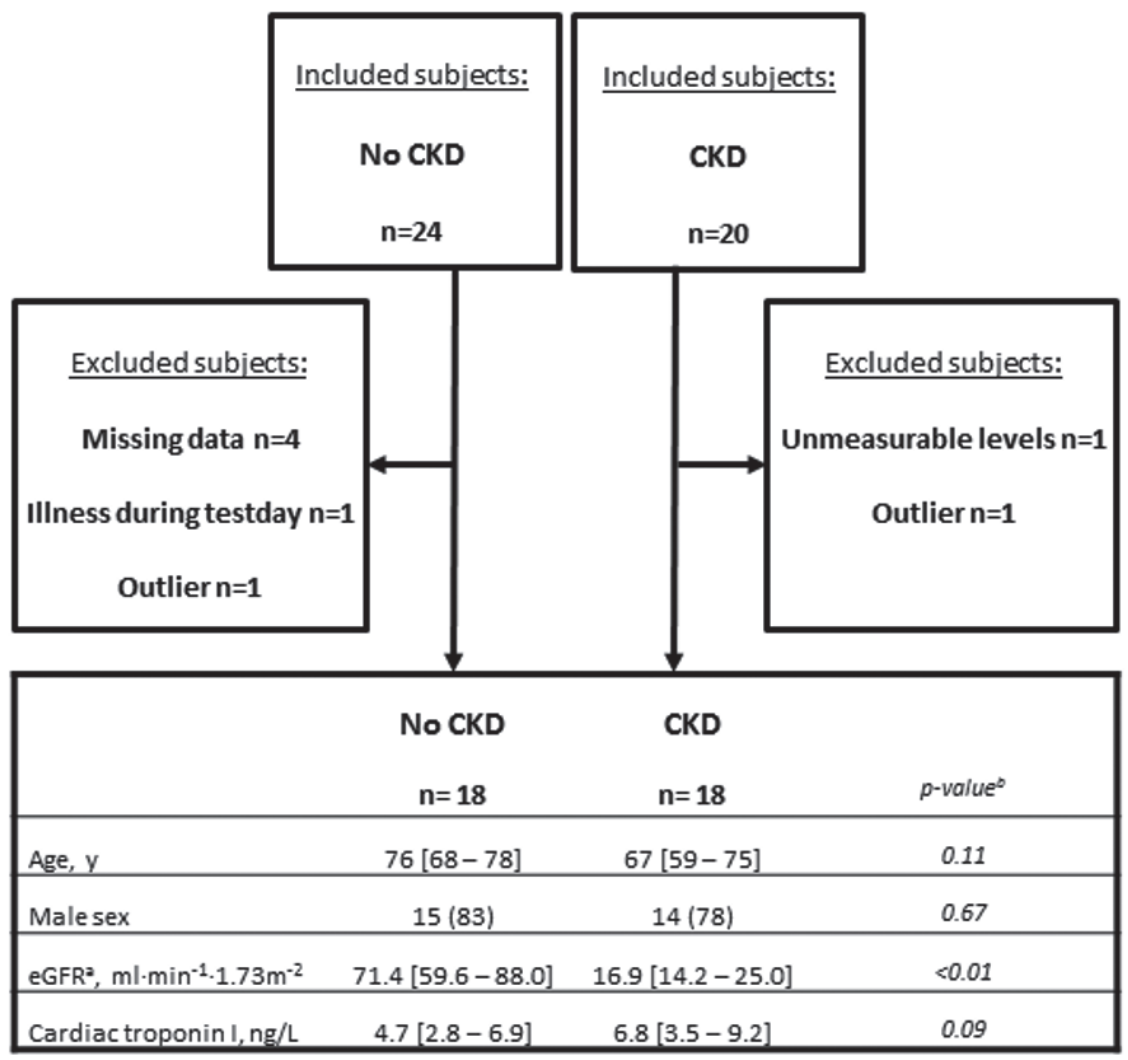

eGFR, estimated glomerular filtration rate according to the CKD-EPI Creatinine-Cystatin C equitation (23) b Comparison between subjects with or without CKD 
From a clinical point of view, the finding of a substantial $\mathrm{CV}_{\mathrm{G}}$ and a low index of individuality emphasizes the wide variety in cardiac troponin concentrations among the population, the limited utility of population-based reference intervals and the importance of the use of delta-change values $(13,32)$. Nevertheless, it is important to consider that the recent development of increasingly short time intervals between serial measurements and detection of small delta-change values, will come to a stop at the point where the changes are so small that there is no distinction more with the physiological variation within a person. This can only be partially overcome by increasing the sensitivity and precision of the assay: Based on a $\mathrm{CV}_{\mathrm{I}}$ of approximately $8 \%$, the RCV may decrease from approximately $37 \%$ to $22 \%$ for cTnI values in the lower range, but will not drop below this $22 \%$.

Figure 6.3. No diurnal pattern in CTnI variation in subjects with or without CKD

no CKD

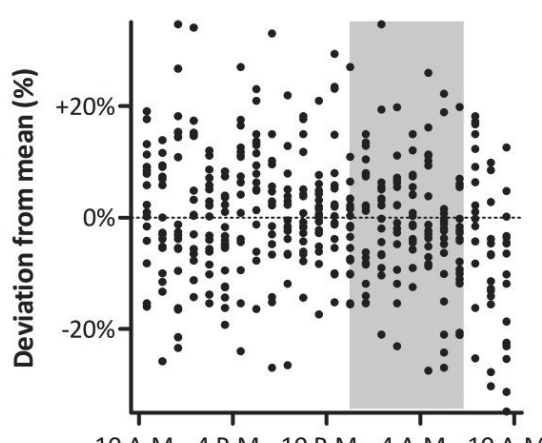

10 A.M. 4 P.M. 10 P.M. 4 A.M. 10 A.M.

Time
CKD

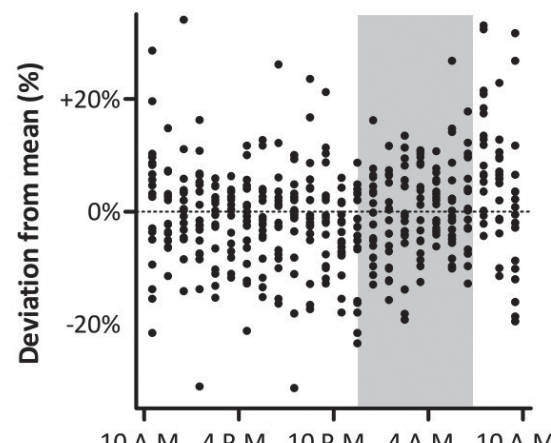

10 A.M. 4 P.M. 10 P.M. 4 A.M. 10 A.M.

Time

We calculated the deviation (in \%) from the mean concentration (over 24h) of each individual on every time point. This variation is random and is not subject to a circadian pattern.

Potential limitations of our study merit consideration. First, most subjects included in the final data analyses have relatively low concentrations of CTnI, therefore it is hard to extrapolate our findings to higher concentration ranges. However, when cTnI values at presentation are much higher, serial measurements are not relevant anymore $(1,3)$. Second, since the prevalence of comorbidities in both populations is relatively high (20), we cannot make an unbiased comparison between health and disease. Therefore, we were only able to examine the effect of the presence of chronic kidney disease. 
In conclusion, with this biological variation study we were the first to examine the hour-to-hour biological variation in cTnI over 24-hours. We showed a random variation over the day. Except from the analytical variation, variation components were of the same magnitude in subjects with or without CKD. Our findings imply that the $\mathrm{CV}_{\mathrm{I}}$ of cardiac troponin I is not affected by the presence of chronic kidney disease. 


\section{References}

1. Roffi M, Patrono C, Collet JP, Mueller C, Valgimigli M, Andreotti F, et al. 2015 ESC Guidelines for the management of acute coronary syndromes in patients presenting without persistent ST-segment elevation: Task Force for the Management of Acute Coronary Syndromes in Patients Presenting without Persistent ST-Segment Elevation of the European Society of Cardiology (ESC). Eur Heart J. 2016;37(3):267-315.

2. Amsterdam EA, Wenger NK, Brindis RG, Casey DE, Jr., Ganiats TG, Holmes DR, Jr., et al. 2014 AHA/ACC Guideline for the Management of Patients with Non-ST-Elevation Acute Coronary Syndromes: a report of the American College of Cardiology/American Heart Association Task Force on Practice Guidelines. J Am Coll Cardiol. 2014;64(24):e139-228.

3. Mueller-Hennessen M, Mueller C, Giannitsis E, Biener M, Vafaie M, deFilippi CR, et al. Serial Sampling of High-Sensitivity Cardiac Troponin T May Not Be Required for Prediction of Acute Myocardial Infarction Diagnosis in Chest Pain Patients with Highly Abnormal Concentrations at Presentation. Clin Chem. 2017;63(2):542-51.

4. Body R, Burrows G, Carley S, Cullen L, Than M, Jaffe AS, et al. High-sensitivity cardiac troponin t concentrations below the limit of detection to exclude acute myocardial infarction: a prospective evaluation. Clin Chem. 2015;61(7):983-9.

5. Sandoval Y, Smith SW, Shah AS, Anand A, Chapman AR, Love SA, et al. Rapid Rule-Out of Acute Myocardial Injury Using a Single High-Sensitivity Cardiac Troponin I Measurement. Clin Chem. 2017;63(1):369-76.

6. Boeddinghaus J, Reichlin T, Nestelberger T, Twerenbold R, Meili Y, Wildi K, et al. Early diagnosis of acute myocardial infarction in patients with mild elevations of cardiac troponin. Clin Res Cardiol. 2017.

7. Nestelberger T, Wildi K, Boeddinghaus J, Twerenbold R, Reichlin T, Gimenez MR, et al. Characterization of the observe zone of the ESC 2015 high-sensitivity cardiac troponin 0h/1halgorithm for the early diagnosis of acute myocardial infarction. Int J Cardiol. 2016;207:23845.

8. Twerenbold R, Wildi K, Jaeger C, Gimenez MR, Reiter M, Reichlin T, et al. Optimal Cutoff Levels of More Sensitive Cardiac Troponin Assays for the Early Diagnosis of Myocardial Infarction in Patients With Renal Dysfunction. Circulation. 2015;131(23):2041-50.

9. de Lemos JA. Increasingly sensitive assays for cardiac troponins: a review. JAMA. 2013;309(21):2262-9.

10. Fraser C. Biological Variation: From principles to practice. Washington: AACC press; 2001.

11. Braga F, Panteghini M. Generation of data on within-subject biological variation in laboratory medicine: An update. Crit Rev CI Lab Sci. 2016;53(5):313-25.

12. Fraser CG. Reference change values. Clin Chem Lab Med. 2011;50(5):807-12.

13. Harris EK. Effects of intra- and interindividual variation on the appropriate use of normal ranges. Clin Chem. 1974;20(12):1535-42.

14. Reichlin T, Schindler C, Drexler B, Twerenbold R, Reiter M, Zellweger C, et al. One-hour ruleout and rule-in of acute myocardial infarction using high-sensitivity cardiac troponin T. Arch Intern Med. 2012;172(16):1211-8.

15. Rubini Gimenez M, Twerenbold R, Jaeger C, Schindler C, Puelacher C, Wildi K, et al. One-hour rule-in and rule-out of acute myocardial infarction using high-sensitivity cardiac troponin I. Am J Med. 2015;128(8):861-70 e4.

16. Wu AH, Akhigbe P, Wians F. Long-term biological variation in cardiac troponin I. Clin Biochem. 2012;45(10-11):714-6. 
17. Wu AH, Shea E, Lu QT, Minyard J, Bui K, Hsu JC, et al. Short- and long-term cardiac troponin I analyte stability in plasma and serum from healthy volunteers by use of an ultrasensitive, single-molecule counting assay. Clin Chem. 2009;55(11):2057-9.

18. Simpson AJ, Potter JM, Koerbin G, Oakman C, Cullen L, Wilkes GJ, et al. Use of observed withinperson variation of cardiac troponin in emergency department patients for determination of biological variation and percentage and absolute reference change values. Clin Chem. 2014;60(6):848-54.

19. Apple FS, Collinson PO, Biomarkers ITFoCAoC. Analytical characteristics of high-sensitivity cardiac troponin assays. Clin Chem. 2012;58(1):54-61.

20. Klinkenberg LJ, Wildi K, van der Linden N, Kouw IW, Niens M, Twerenbold R, et al. Diurnal Rhythm of Cardiac Troponin: Consequences for the Diagnosis of Acute Myocardial Infarction. Clin Chem. 2016;62(12):1602-11.

21. Bartlett WA, Braga F, Carobene A, Coskun A, Prusa R, Fernandez-Calle P, et al. A checklist for critical appraisal of studies of biological variation. Clin Chem Lab Med. 2015;53(6):879-85.

22. Levey AS, Stevens LA, Coresh J. Conceptual model of CKD: applications and implications. Am J Kidney Dis. 2009;53(3 Suppl 3):S4-16.

23. Inker LA, Schmid CH, Tighiouart $\mathrm{H}$, Eckfeldt JH, Feldman HI, Greene T, et al. Estimating glomerular filtration rate from serum creatinine and cystatin C. N EnglJ Med. 2012;367(1):20-9.

24. Fraser CG, Harris EK. Generation and application of data on biological variation in clinical chemistry. Crit Rev Clin Lab Sci. 1989;27(5):409-37.

25. Roraas T, Petersen PH, Sandberg S. Confidence Intervals and Power Calculations for Within-Person Biological Variation: Effect of Analytical Imprecision, Number of Replicates, Number of Samples, and Number of Individuals (vol 58, pg 1306, 2012). Clinical Chemistry. 2012;58(12):1724-.

26. Burdick RK GF. Confidence intervals on variance components. New York: Marcel Dekker, Inc; 1992.

27. Petersen PH, Fraser CG, Sandberg S, Goldschmidt H. The index of individuality is often a misinterpreted quantity characteristic. Clin Chem Lab Med. 1999;37(6):655-61.

28. Ricos C, Iglesias N, Garcia-Lario JV, Simon M, Cava F, Hernandez A, et al. Within-subject biological variation in disease: collated data and clinical consequences. Ann Clin Biochem. 2007;44(Pt 4):343-52.

29. Aakre KM, Roraas T, Petersen PH, Svarstad E, Sellevoll H, Skadberg O, et al. Weekly and 90Minute Biological Variations in Cardiac Troponin T and Cardiac Troponin I in Hemodialysis Patients and Healthy Controls. Clinical Chemistry. 2014;60(6):838-47.

30. Mbagaya W, Luvai A, Lopez B. Biological variation of cardiac troponin in stable haemodialysis patients. Ann Clin Biochem. 2015;52(Pt 5):562-8.

31. Tonelli M, Wiebe N, Guthrie B, James MT, Quan H, Fortin M, et al. Comorbidity as a driver of adverse outcomes in people with chronic kidney disease. Kidney Int. 2015;88(4):859-66.

32. Harris EK. Statistical aspects of reference values in clinical pathology. Prog Clin Pathol. 1981;8:45-66. 


\section{Supplemental information}

\section{Supplemental figure 6.1. Individual diurnal cardiac troponin I profiles}

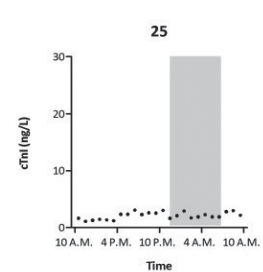

29

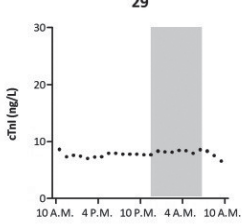

Time

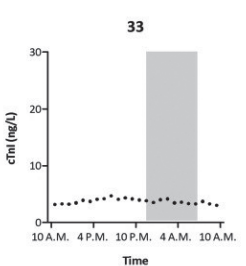

38

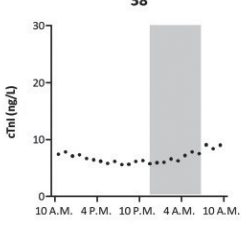

Time

42

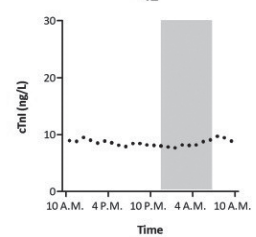

26

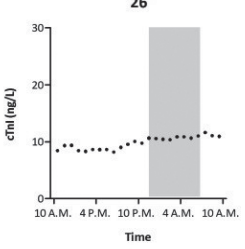

30

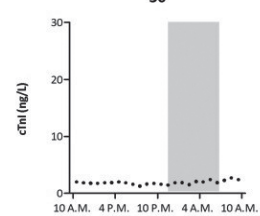

Time

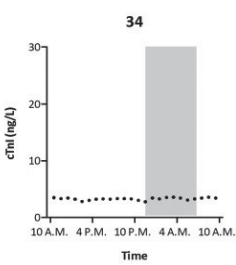

39

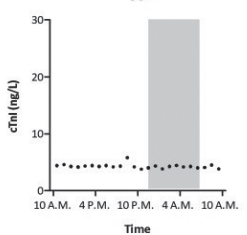

43

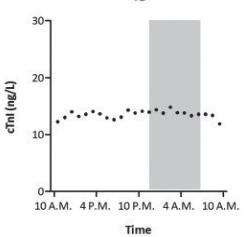

27

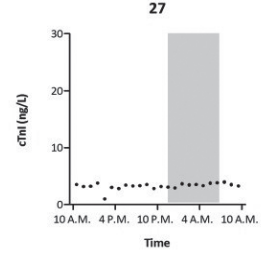

31

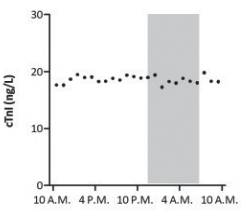

Time

35

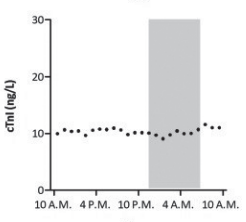

Time

40

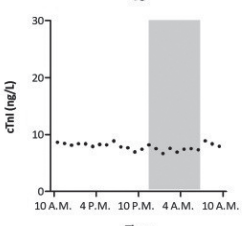

Time

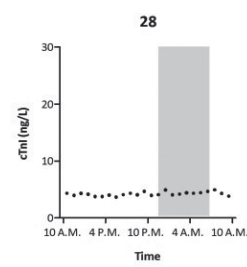

32

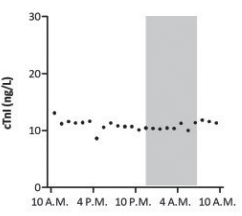

Time

37

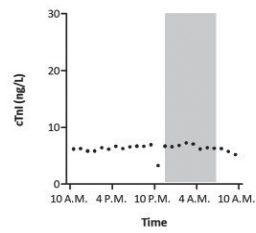

41

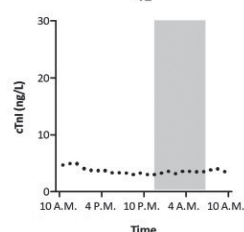

Depicted are the mean of the duplicate measurements of subjects with chronic kidney disease. The numbering is identical to Figure 1. Subjects slept in a supine position between 11:30 P.M. and 7:00 A.M. (shaded area) 


\section{Chapter 7}




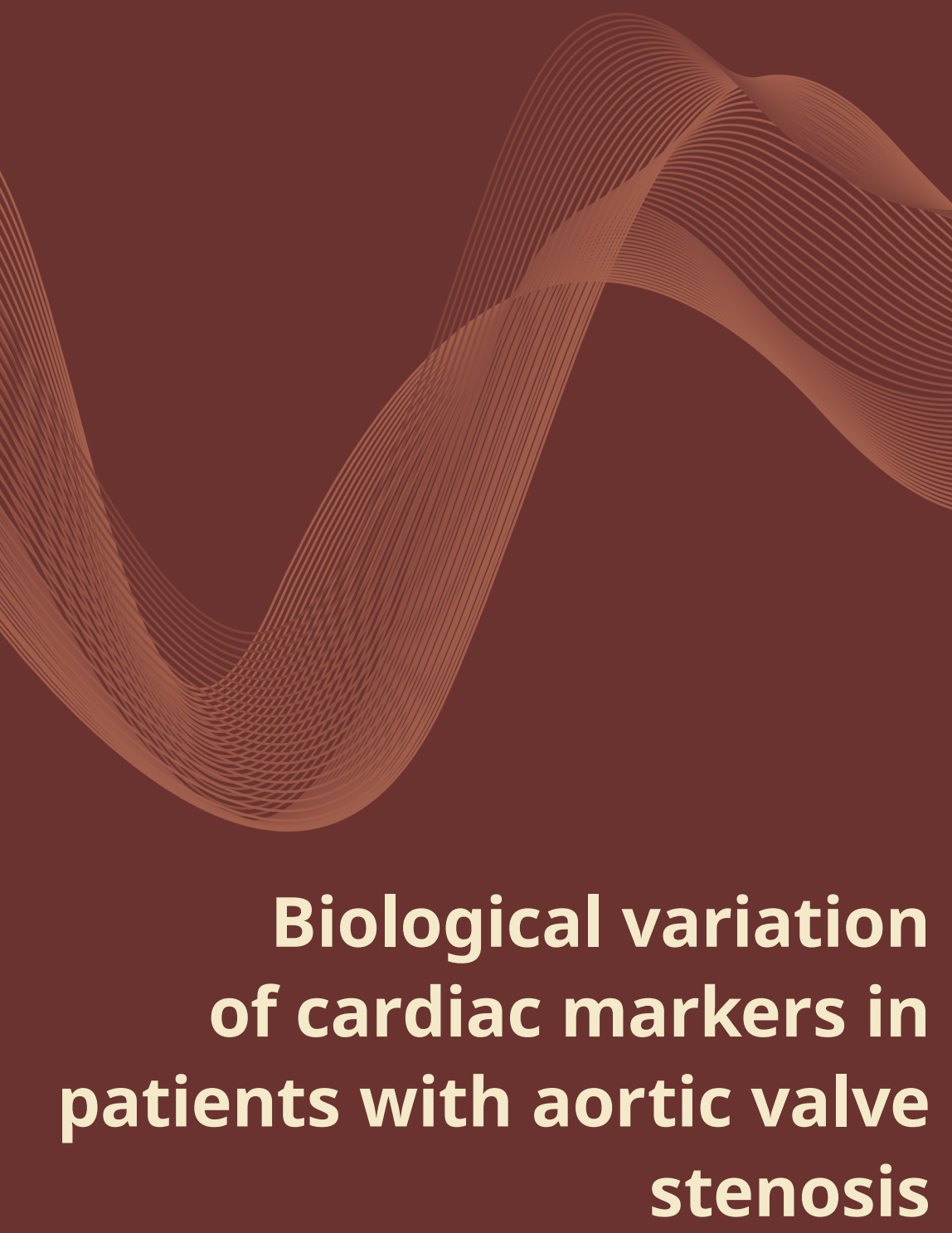

Frederique E.C.M. Peeters, Bas L.J.H. Kietselaer, Judith M. Hilderink, Noreen van der Linden, Marijke Niens, Harry J.G.M. Crijns, Steven J.R. Meex 


\section{Abstract}

\section{Background}

Incorporation of cardiac biomarkers hold potential to be of value in the follow up and management of aortic valve stenosis. However, we lack knowledge about the biological variation of these biomarkers, which is an essential component of correct interpretation of serial biomarker management. Therefore, the aim of the current study was to determine the biological variation of a panel of cardiac biomarkers in subjects with stable aortic valve stenosis.

\section{Methods}

Serial blood sampling was performed in 25 subjects with (echocardiographic confirmed) moderate aortic valve stenosis (echocardiographic confirmed), all free from acute cardiovascular events in the past six months. Each subject visited the outpatient clinic seven times during one year, and blood samples were taken under standardized conditions. Analytical variation $\left(\mathrm{CV}_{\mathrm{A}}\right)$, within-subject biological variation $\left(C V_{I}\right)$, between-subject biological variation $\left(C_{G}\right)$, index of individuality (II) and reference change values (RCV) were calculated for brain natriuretic peptide (BNP), NT-pro BNP, high-sensitivity troponin T and I and ST2 (suppression of tumourigenicity 2 ).

\section{Results}

Within-subject biological variation was highest for BNP $(62.03 \%, 95 \%$ CI 17.05;23.43) and lowest for troponin I $(9.18 \%, 95 \%$ CI 2.83;13.75). Between-subject biological variation was considerably higher in all biomarkers except BNP, and ranged from 19.82\% (95\% CI 13.76;33.36) for ST2 to $57.19 \%$ (95\% CI 40.39;97.29) for troponin-T. NT-pro BNP, troponin-T and ST2 revealed analytical variation $<5 \%$, whilst BNP and troponin-I showed a higher $\mathrm{CV}_{\mathrm{A}}$ (19.74 and 14.86 respectively). All biomarkers except BNP showed marked individuality, with II ranging from 0.21 to 0.67 (BNP 1.34).

\section{Conclusion}

Biological variation indices of subjects with stable aortic valve stenosis approximate those of healthy subjects.

\section{Key words}

Aortic valve stenosis, progression, cardiac biomarkers, biological variation, echocardiography 


\section{Introduction}

Being indicators of biological processes, circulating biomarkers are commonly used in clinical decision making for diagnosing, risk stratification and management of various cardiovascular diseases $(1,2)$. However, incorporation of biomarkers in the management of aortic valve stenosis (AVS), the most common type of valvular disease in the Western world, is a matter of debate. The 2017 ESC/EACTS guidelines recommend to incorporate repeated measurements of only one markedly elevated biomarker: B-type natriuretic peptide (BNP) in asymptomatic severe AVS forms a class IIa (level C) indication for surgical or trans-catheter valve replacement, while the 2014 AHA/ACC guidelines do not make reference to biomarkers in the recommendations $(3,4)$. Once present, AVS is a progressive disease with poor understanding of exact underlying mechanisms, and echocardiography forms the golden standard for diagnosis and evaluation (5). Parameters used during echocardiography provide limited insight in the pathophysiology though, and are poor predictors for progression rates (6). Therefore, biomarkers hold potential as a complementary approach to gain insight in AVS progression and timing of intervention. Theoretically, serial measurements of biomarkers showing changes over time in parallel with AVS progression are of potential use in tailored AVS management. However, to interpret whether changes over time are 'real' and not just a physiological fluctuation, knowledge on the magnitude of physiological variation of a biomarker is essential. This principle is known as biological variation (7). Biological variation assumes that biomarker concentrations fluctuate around a homeostatic set point, driven by withinsubject and analytical variation ( $\mathrm{CV}_{\mathrm{I}}$ and $\mathrm{CV}_{\mathrm{A}}$ respectively). These components enable estimation of the reference change value (RCV), which represents the required threshold for a change between measurements to be statistically significant and with that, a ' true' change (8). (2) Additionally, the variation between specific set points, known as the index of individuality (II), can be calculated $(9,10)$.

Studies investigating biological variation of cardiac biomarkers have been performed in a few specific patient populations for instance with heart failure or kidney dysfunction, but remain sparse to date (11-16). Biological variation of biomarkers in patients with aortic valve stenosis has not been determined.

Several cardiac biomarkers have been investigated in AVS, such as BNP (brain natriuretic peptide), the N-terminal fragment of BNP (NT-pro BNP), troponin-T and -I and ST2 ST2 (suppression of tumourigenicity 2) with variable results (17). Taking the potential role of biomarkers in the course of AVS into account, it would be of 
importance to gain insight in the variation of biomarkers for the interpretation of serial biomarker results. Therefore, the aim of the current study was to assess the analytical and biological variation of cardiac biomarkers BNP, NT-pro BNP, troponin-T, troponin-I and ST2 in patients with AVS.

\section{Methods}

\section{Study population}

The study population consisted of 25 subjects (>18 years) with known moderate aortic valve stenosis who were followed up at the outpatient clinics in the department of Cardiology in the Maastricht University Medical Center (MUMC), the Netherlands. Severity of aortic valve stenosis was defined by echocardiographic measurement (mean gradient 20-40 mmHg, AVA 1.0-1.5 cm2 or maximal transvalvular velocity 3-3.9 $\mathrm{m} / \mathrm{s}$ ). Prior to inclusion, subjects had to be in a stable medical condition and without complaints directly related to aortic valve stenosis. Exclusion criteria consisted of presence of severe aortic valve stenosis, left ventricular ejection fraction (LVEF) $<50 \%$, documented atrial fibrillation in the last year, chronic kidney disease (eGFR $<45 \mathrm{~mL} / \mathrm{min} / 1.73 \mathrm{~m}^{2}$ ) and a history of acute myocardial infarction, hospitalization for heart failure or a pulmonary embolism within 6 months prior to inclusion. Subjects who met any of the exclusion criteria and those unable to provide written informed consent were not included. At the end of the study period, subjects were evaluated to monitor progression of aortic valve stenosis and indication for surgical intervention. Subjects with symptomatic aortic valve stenosis and those showing an increase in mean gradient $>7 \mathrm{mmHg}$ or maximum velocity $>0.3 \mathrm{~m} / \mathrm{s}$ were reported as progressive $(18,19)$. This study was performed according to the Declaration of Helsinki and was approved by the local Institutional Review Board. It was registered at www.clinicaltrials.gov as NCT02510482. All study subjects provided written informed consent. This study was executed according to the critical appraisal checklist criteria for biological variation studies by Bartlett et al (20).

\section{Study design}

All subjects visited the outpatient clinics of Cardiology of our center 7 times during 1 year (baseline and 1 day, 1 week, 1 month, 3 months, 6 months and 12 months after baseline respectively). Visits and blood samplings were performed in a standardized manner and all patients were asked to refrain from intense physical heavy physical labor and exercise training 2 days before each visit. All patient visits took place between 08.00 and $09.00 \mathrm{AM}$, during which standard history taking and 
a standardized questionnaire (including medication use) were performed. Blood sampling was performed through standard venipuncture in seated position.

\section{Laboratory measurements}

Blood samples were collected in serum, ethylenediaminetraacetic acid (EDTA) and citrate tubes. Immediately after collection, standard hematological parameters (hemoglobin, hematocrit, white blood cells, neutrophils) were measured in EDTAsamples using the Sysmex XE-5000 analyzer (Sysmex Corporation, Kobe, Japan). After this measurement (approx. 5 minutes), EDTA samples were centrifuged $(12 \mathrm{~min}, 2500 \mathrm{~g})$, and were aliquoted. Citrate samples were centrifuged together with the EDTA samples, and were additionally centrifuged (10 min, $10.000 \mathrm{~g}$ ). The serum samples were centrifuged after 25 minutes (12 min, $2500 \mathrm{~g}$ ). Directly after aliquoting, samples were stored at $-80^{\circ} \mathrm{C}$ until further analyses.

The estimated glomerular filtration rate (eGFR) was calculated according to the Chronic Kidney Disease Epidemiology Collaboration (CKD-EPI) formula (21).

NT-pro BNP and high-sensitivity troponin-T levels were measured on the COBAS 6000 analyzer, and measurements of high-sensitivity troponin-I were measured with the STAT high-sensitivity troponin I assay (Architect, Abbott Diagnostics). BNP was measured on the Architect analyzer (Abbott Diagnostics) and ST2 using the Presage ${ }^{\circledR}$ ST2 Assay (Critical Diagnostics). To estimate analytical variation, all samples of BNP, NT-pro BNP, troponin-T and troponin-I were measured in duplicate. $61 \%$ of samples of ST2 were measured in duplicate.

\section{Echocardiography}

Standard two-dimensional transthoracic echocardiography was performed by an independent observer prior to inclusion and during regular visits to the outpatient clinics (Sonos 5500 and IE33, Philips Medical Systems, Andover, MA, USA) according to the European Association of Echocardiography (EAE) guidelines (22).

\section{Statistical analyses}

Cochran's C test was used to test data for homogeneity in analytical and withinsubject biological variances as suggested by Fraser and Harris (23). In short, variances were tested for homogeneity by examination of the (maximum variance): (sum of variances)-ratio and compared with a prior determined critical value for both analytical and within-subject biological variances $(23,24)$. Subjects were excluded 
until homogeneity of variances was achieved. Between-subject outliers were identified using the criteria of Reed $(7,25,26)$. Between-subject $\left(C_{G}\right)$ and withinperson subject biological variation $\left(\mathrm{CV}_{\mathrm{I}}\right)$ and analytical variation $\left(\mathrm{CV}_{\mathrm{A}}\right)$ were calculated using a balanced analysis of variance with a nested random design in two levels (27). The method of Burdick and Graybill was used accordingly to calculate $95 \%$ confidence intervals (CI) of the variance components $(27,28)$. The index of individuality (II) and Reference Change Value (RCV) were calculated according to the method described by Petersen et al. and Fraser and Harris $(23,29,30)$. II was calculated using the formula:

$$
\mathrm{II}=\frac{\sqrt{C V_{A}^{2}+C V_{I}^{2}}}{C V_{G}}
$$

The RCV was calculated using the formula:

$$
R C V=\sqrt{2} * Z * \sqrt{C V_{A}^{2}+C V_{I}^{2}}
$$

In this formula, $\mathrm{Z}$ represents the number of standard deviations appropriate for the desired level of statistical significance for a bidirectional change. For RCV calculations in this study, a Z-score of 1.96 was used. Additionally, RCVs were calculated and evaluated after log-normal transformation (31). All statistical analyses were performed using SPSS statistics version 22 (IBM Corp, Armonk, NY).

\section{Results}

\section{Baseline population characteristics}

A total of 25 subjects with moderate aortic valve stenosis participated in the current study. Mean age $( \pm S D)$ was $66 \pm 6$ years, and $44 \%(n=11)$ subjects were female. All subjects had moderate aortic valve stenosis on baseline echocardiography (median [IQR] mean gradient 25 [11] mmHg, maximum velocity $340[65] \mathrm{cm} / \mathrm{s}$ and aortic valve area $1.3[0.2] \mathrm{cm}^{2}$ ) and median [IQR] left ventricular ejection fraction (LVEF) 63 [5]\%. None had complaints attributable to aortic valve stenosis. Baseline concentrations of all biomarkers are shown in figure 7.1.

\section{Biological variation in stable aortic valve stenosis}

The ranges observed in biomarkers in all subjects (BNP, NT-pro BNP, troponin-T, troponin-I and ST2) are plotted in figure 7.2. Sample collection was complete for all subjects. Figure 1 shows the baseline characteristics of the total population. 
In 9 subjects, aortic valve stenosis was progressive (figure 7.1). Therefore, the primary analysis to determine biological variation was performed in the stable population $(n=16)$. An overview of outliers and excluded subjects per biomarker is provided in Supplemental table 7.1.

Within-subject variation $\left(\mathrm{CV}_{\mathrm{I}}\right)$ and between-subject variation $\left(\mathrm{CV}_{\mathrm{G}}\right)$ of $\mathrm{BNP}, \mathrm{NT}$-pro BNP, troponin-T, troponin-I and ST2 were determined accordingly. NT-pro BNP, troponin-T, troponin-I and ST2 revealed lower within-subject than between-subject values. BNP showed highest within-subject variation $(62.03 \%, 95 \%$ CI 17.05;23.43) and troponin-I lowest $(9.18 \%, 95 \%$ CI 2.83;13.75). Between-subject biological variation was considerably higher in all biomarkers (except BNP), and ranged from 19.82\% (95\% CI 13.76;33.36) for ST2) to 57.19\% (95\% CI 40.39;97.29) for troponin-T, respectively. NT-pro BNP, troponin-T and $\mathrm{ST} 2$ revealed $\mathrm{CV}_{\mathrm{A}}<5 \%$ (ranging from $1.90 \%$ to $3.67 \%$ ), whereas BNP and troponin-I showed higher analytical variation (19.74 and 14.86 respectively).

Additionally, the variation between specific set points, known as the index of individuality (II) was calculated. All biomarkers except BNP showed marked individuality, with II ranging from 0.21 to 0.67 (BNP 1.34). Table 7.1 depicts biological variation parameters of all biomarkers, including RCV.

Biological variation of the total population: does progressive aortic valve stenosis change biological variation indices?

A secondary, explorative analysis was performed to determine biological variation in the total population ( $n=25$ ) thus including the subpopulation of subjects showing progressive aortic valve disease $(n=9)$. Of the last group, 5 patients progressed to symptomatic aortic valve stenosis after their 6 months visit. Another 4 remained asymptomatic, but showed progressive disease during echocardiographic examination after 1 year of follow up. Supplemental table 7.2 provides an overview of outliers and excluded subjects per biomarker. Consecutively, variation components were calculated for the total population, which are presented in Table 7.2.

BNP showed a higher between-subject variation and lower RCV and index of individuality were observed in the total population. NT-pro BNP showed a marked increase in between- and within-subject variation and the latter contributed to the increased RCV observed in this group. For troponin-I the within-subject variability and RCV increased markedly. At last, between-subject variability of ST2 increased 
and with similar within-subject variation in both groups, the index of individuality of ST2 decreased in the total population.

Figure 7.1: Flowchart of baseline and follow-up populations, including baseline characteristics.

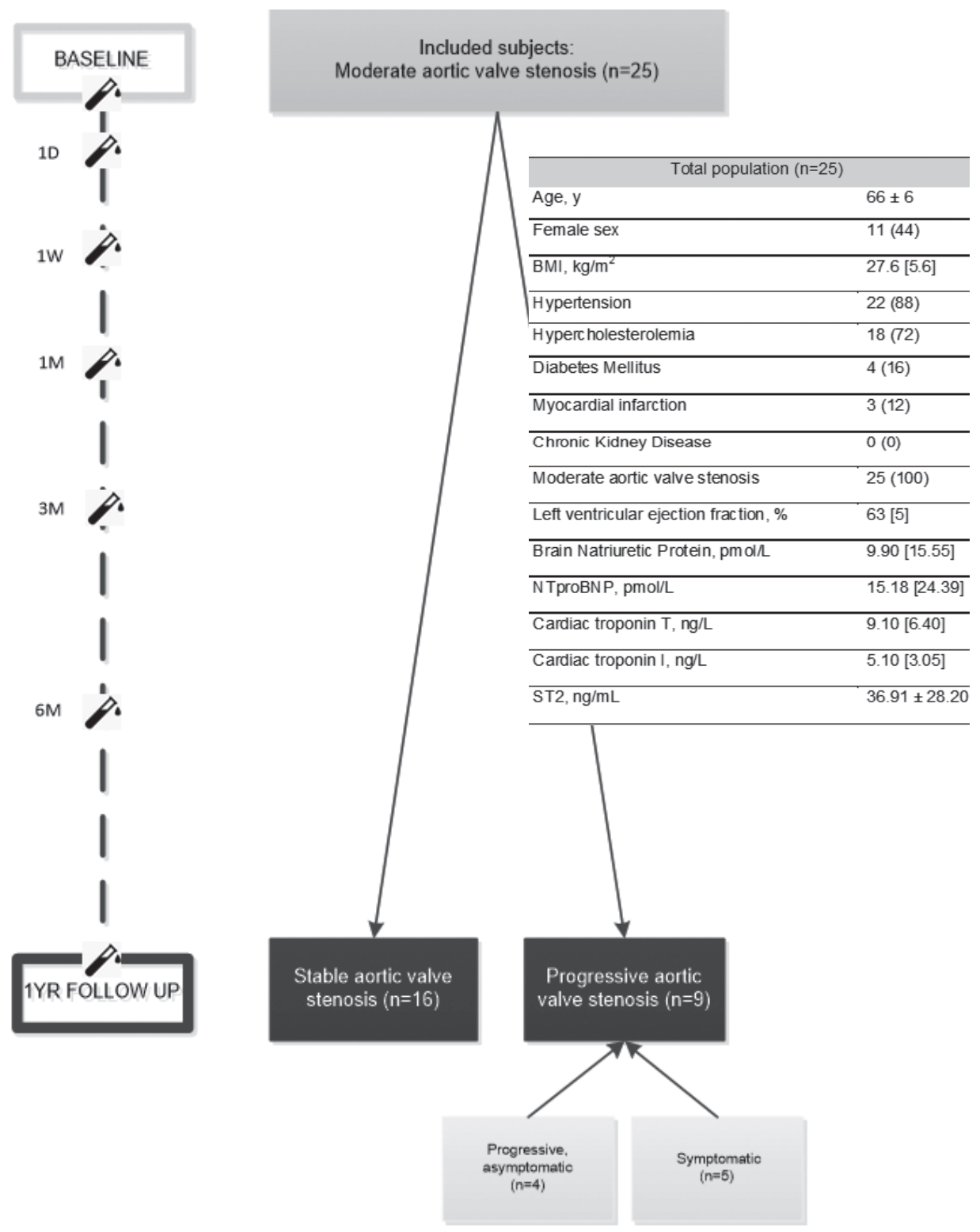

Continuous variables are expressed as mean \pm SD or median [IQR] depending on their distribution. Categorical variables are reported as $\mathrm{n}(\%)$. 
Figure 7.2: Subject-specific concentrations of BNP, NT-pro BNP, troponin-T (hsTnT), troponin-I (hsTnI) and ST2 over 7 measurement point during 1 year of follow-up.
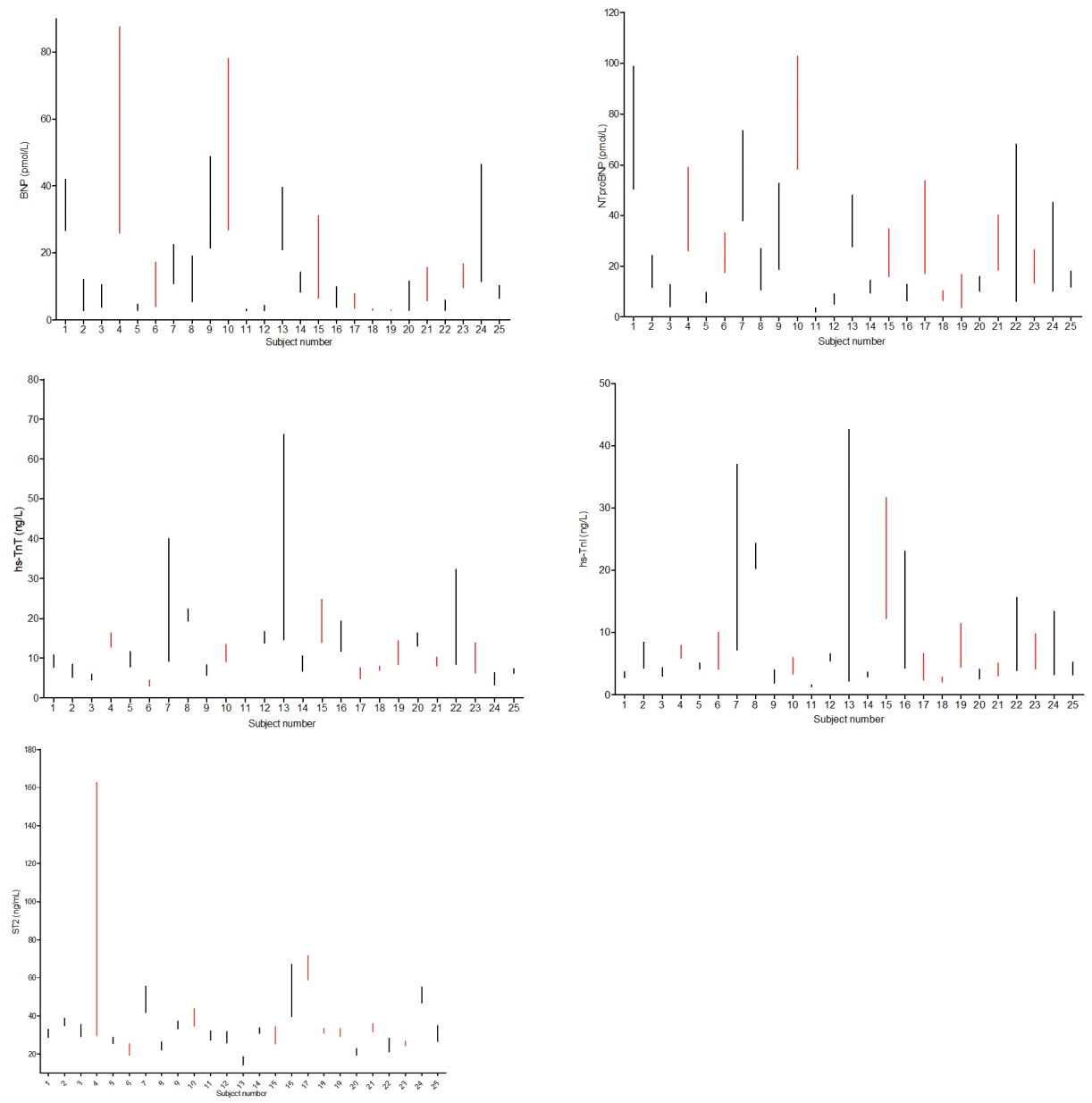

Parameters are shown in absolute ranges (minimum-maximum concentration). Red lines indicate subjects with progressive AVS 
Table 7.1: Analytical variation, biological variation, indexes of individuality and reference change values of all measured biomarkers in stable aortic valve stenosis.

\begin{tabular}{|c|c|c|c|c|c|c|c|}
\hline & $\begin{array}{l}\text { Mean } \\
\text { concentration }\end{array}$ & Vari & ance compon & ents & & $\mathrm{RCV}^{\mathrm{b}}$ & II \\
\hline & & $\mathrm{CV}_{\mathrm{G}}$ & $\mathrm{CV}_{\mathrm{I}}$ & $\mathrm{CV}_{\mathrm{A}}{ }^{\mathrm{a}}$ & Normal & Log-normal & \\
\hline BNP & $5.71 \mathrm{pmol} / \mathrm{L}$ & $\begin{array}{l}48.42 \\
(28.62 ; 91.72)\end{array}$ & $\begin{array}{l}62.03 \\
(52.49 ; 75.35)\end{array}$ & $\begin{array}{l}19.74 \\
(17.05 ; 23.43)\end{array}$ & 180.43 & $52.45 ;-34.41$ & 1.34 \\
\hline $\begin{array}{l}\text { NT-pro } \\
\text { BNP }\end{array}$ & $9.27 \mathrm{pmol} / \mathrm{L}$ & $\begin{array}{l}43.03 \\
(27.73 ; 88.81)\end{array}$ & $\begin{array}{l}22.00 \\
(18.33 ; 27.50)\end{array}$ & $\begin{array}{l}1.90 \\
(1.60 ; 2.33)\end{array}$ & 61.20 & $44.62 ;-30.85$ & 0.51 \\
\hline Hs-TnT & $9.19 \mathrm{ng} / \mathrm{L}$ & $\begin{array}{l}57.19 \\
(40.39 ; 97.29)\end{array}$ & $\begin{array}{l}11.22 \\
(9.55 ; 13.51)\end{array}$ & $\begin{array}{l}3.67 \\
(3.19 ; 4.33)\end{array}$ & 32.72 & $16.82 ;-14.40$ & 0.21 \\
\hline Hs-TnI & $3.64 \mathrm{ng} / \mathrm{L}$ & $\begin{array}{l}35.01 \\
(23.31 ; 67.61)\end{array}$ & $\begin{array}{l}9.18 \\
(2.83 ; 13.75)\end{array}$ & $\begin{array}{l}14.86 \\
(12.65 ; 17.99)\end{array}$ & 48.41 & $72.98 ;-42.19$ & 0.50 \\
\hline ST2 & $28.38 \mathrm{ng} / \mathrm{mL}$ & $\begin{array}{l}19.82 \\
(13.76 ; 33.36)\end{array}$ & $\begin{array}{l}13.13 \\
(11.33 ; 15.60)\end{array}$ & $\begin{array}{l}2.01 \\
(1.72 ; 2.41)\end{array}$ & 36.83 & * & 0.67 \\
\hline
\end{tabular}

Values are \% $(95 \% \mathrm{CI}) ; \mathrm{CI}=$ confidence interval; $\mathrm{CV}_{\mathrm{A}}=$ analytical coefficient of variation; $\mathrm{CV}_{\mathrm{G}}=$ between-subject coefficient of variation; $\mathrm{CV}_{\mathrm{I}}=$ within-subject biological coefficient of variation; RCV: reference change value. BNP: brain natriuretic peptide; NT-pro-BNP: N-terminus probrain natriuretic peptide; hs-TnT: high-sensitivity troponin T; hs-TnI: high-sensitivity troponin I; IST2: suppression of tumourigenicity 2. II: index of individuality. ${ }^{\text {a }}$ On the basis of duplicate measurements. ${ }^{\mathrm{b}}$ On the basis of a Z-score of 1.96. *Normal distribution

Table 7.2: Analytical variation, biological variation, indexes of individuality and reference

\begin{tabular}{|c|c|c|c|c|c|c|c|}
\hline & \multirow{2}{*}{$\begin{array}{l}\text { Mean } \\
\text { concentration }\end{array}$} & \multicolumn{3}{|c|}{ Variance components } & \multicolumn{2}{|c|}{$\mathrm{RCV}^{\mathrm{b}}$} & \multirow[t]{2}{*}{ II } \\
\hline & & $\mathrm{CV}_{\mathrm{G}}$ & $\mathrm{CV}_{\mathrm{I}}$ & $\mathrm{CV}_{\mathrm{A}}{ }^{\mathrm{a}}$ & Normal & Log-normal & \\
\hline BNP & $9.31 \mathrm{pmol} / \mathrm{L}$ & $\begin{array}{l}86.28 \\
(64.91 ; 126.97)\end{array}$ & $\begin{array}{l}37.64 \\
(33.18 ; 43.37)\end{array}$ & $\begin{array}{l}12.25 \\
(10.97 ; 13.87)\end{array}$ & 109.73 & $52.01 ;-34.21$ & 0.46 \\
\hline $\begin{array}{l}\text { NT-pro } \\
\text { BNP }\end{array}$ & $23.68 \mathrm{pmol} / \mathrm{L}$ & $\begin{array}{l}75.83 \\
(58.49 ; 106.41)\end{array}$ & $\begin{array}{l}38.42 \\
(34.52 ; 43.34)\end{array}$ & $\begin{array}{l}2.01 \\
(1.82 ; 2.25)\end{array}$ & 106.65 & $42.91 ;-30.03$ & 0.51 \\
\hline Hs-TnT & $9.17 \mathrm{ng} / \mathrm{L}$ & $\begin{array}{l}48.59 \\
(37.01 ; 70.40)\end{array}$ & $\begin{array}{l}14.20 \\
(12.58 ; 16.27)\end{array}$ & $\begin{array}{l}3.86 \\
(3.46 ; 4.35)\end{array}$ & 40.79 & $33.67 ;-25.19$ & 0.30 \\
\hline Hs-TnI & $4.37 \mathrm{ng} / \mathrm{L}$ & $\begin{array}{l}34.45 \\
(25.06 ; 52.70)\end{array}$ & $\begin{array}{l}23.79 \\
(20.68 ; 27.79)\end{array}$ & $\begin{array}{l}10.57 \\
(9.41 ; 12.06)\end{array}$ & 72.17 & $65.67 ;-39.64$ & 0.76 \\
\hline ST2 & $30.80 \mathrm{ng} / \mathrm{mL}$ & $\begin{array}{l}32.13 \\
(24.71 ; 45.65)\end{array}$ & $\begin{array}{l}10.67 \\
(9.53 ; 12.12)\end{array}$ & $\begin{array}{l}2.07 \\
(1.82 ; 2.39)\end{array}$ & 30.13 & * & 0.34 \\
\hline
\end{tabular}

Values are \% $(95 \% \mathrm{CI}) ; \mathrm{CI}=$ confidence interval; $\mathrm{CV}_{\mathrm{A}}=$ analytical coefficient of variation; $\mathrm{CV}_{\mathrm{G}}=$ between-subject coefficient of variation; $\mathrm{CV}_{\mathrm{I}}=$ within-person biological coefficient of variation; $\mathrm{RCV}=$ reference change value

BNP: brain natriuretic peptide; NT-pro-BNP: N-terminus pro-brain natriuretic peptide; hs-TnT: high-sensitivity troponin T; hs-TnI: high-sensitivity troponin I; ST2: suppression of tumourigenicity 2. II: index of individuality

${ }^{a}$ On the basis of duplicate measurements

${ }^{b}$ On the basis of a Z-score of 1.96

*Normal distribution 


\section{Discussion}

Several (combinations of) biomarkers are associated with adverse outcome in patients with aortic valve stenosis and are suggested to be of value in future clinical care (32-35). Ideally; changes in serial measurements of a biomarker should reflect progression. However, to interpret serial measurements, knowledge about variation components of biomarkers is essential.

Therefore, this study is the first to examine biological variation of several cardiac biomarkers (BNP, NT-pro BNP, troponin-T, troponin-I and ST2) in subjects with stable moderate aortic valve stenosis. We report three major findings: First, we found a substantial variation within and between subjects with AVS $\left(\mathrm{CV}_{\mathrm{I}}=\right.$ 9-62\% and $\mathrm{CV}_{\mathrm{G}}=20-58 \%$ ), corresponding with previous studies. Within-subject variability was relatively small for ST2 and troponin-T and I, whilst large withinsubject variation was found for BNP and NT-pro BNP. These results suggest that values of BNP and NT-pro BNP have to vary substantially to hint toward relevant changes, whilst smaller variations in serial measurements in troponin-T and ST2 might indicate a relevant change. Studies examining biological variation have been performed in healthy populations, but a growing interest in variation components of biomarkers in populations with (cardiovascular) disease resulted in newer studies addressing biological variation in heart failure and chronic kidney disease $(11,13$, $16,36)$. We found that indices of biological variation in stable aortic valve stenosis approximated indices found in studies investigating biological variation in healthy subjects and chronic and stable heart failure $(13,16,36)$.

Second, we found substantial differences in various biological variation indices of BNP, NT-pro BNP, troponin-T, troponin-I and ST2 in the population with stable aortic valve stenosis in comparison to our total AVS population, suggesting that progressive aortic valve disease affects biological variation.

Third, between-subject variation was higher than within-subject variation in all biomarkers but BNP in our population. Both affect the index of individuality and therefore, we found low indexes of individuality in all biomarkers (except BNP) and thus marked individuality in our population. Therefore, the use of population based reference values is of limited utility $(8,10,37)$. Instead, the use of reference change values (RCV) is of value in these biomarkers. 
From a clinical perspective, a low index of individuality and marked betweensubject variation underline the extensive ranges of biomarker concentrations found in the population. This underlines the importance to use of reference change values instead of general population-based reference intervals to interpret serial measurements in an individual. However, the individual variation is undervalued in daily practice, as we tend to interpret biomarkers above or below general thresholds to identify a patient with low or high risk. The use of RCVs with serial biomarker measurements bears potential to integrate in the development of tailored treatment strategies in personalized medicine. In the context of interpretation of true changes in serial measurements, a little difference between measurements of biomarkers with a large RCV challenge the interpretation, while a little difference in measurements of biomarkers with a limited RCV suggest a true change. Thus, absolute changes of biomarkers should be placed in perspective and hold potential to improve estimation of the true risk of a patient.

\section{Study limitations}

Potential limitations of the current study merit attention. First, we included 25 patients with stable moderate aortic valve stenosis at baseline for analysis of biological variation. After 1 year of follow up, 9 patients showed progressive disease. Sixteen patients with stable aortic valve stenosis were left for primary analysis, providing us with sufficient power to make reliable estimations for variation components for primary analyses (27). Second, the size of our population did not allow stratification in sex- or- age-groups. Given the potential differences in pathophysiology between men and women, further exploration would be interesting.

\section{Conclusion}

This is the first study to present biological variation of cardiac biomarkers BNP, NT-pro BNP, troponin-T, troponin-I and ST2 in a population with stable aortic valve stenosis. Indices of biological variation approximate those in healthy subjects (derived from previous studies). BNP and NT-pro BNP show a high within-subject variation and RCV, whereas troponin-T, troponin-I and ST2 show lower variation indices. 


\section{References}

1. Toutouzas K, Stathogiannis K, Latsios G, Synetos A, Drakopoulou M, Penesopoulou V, Michelongona A, Tsiamis E, Tousoulis D. Biomarkers in aortic valve stenosis and their clinical significance in transcatheter aortic valve implantation. Curr Med Chem 2017.

2. van Holten TC, Waanders LF, de Groot PG, Vissers J, Hoefer IE, Pasterkamp G, Prins MW, Roest M. Circulating biomarkers for predicting cardiovascular disease risk; a systematic review and comprehensive overview of meta-analyses. PLoS One 2013;8(4):e62080.

3. Baumgartner H, Falk V, Bax JJ, De Bonis M, Hamm C, Holm PJ, Iung B, Lancellotti P, Lansac E, Rodriguez Munoz D, Rosenhek R, Sjogren J, Tornos Mas P, Vahanian A, Walther T, Wendler O, Windecker S, Zamorano JL, Group ESCSD. 2017 ESC/EACTS Guidelines for the management of valvular heart disease. Eur Heart J 2017;38(36):2739-2791.

4. Nishimura RA, Otto CM, Bonow RO, Carabello BA, Erwin JP, 3rd, Guyton RA, O'Gara PT, Ruiz CE, Skubas NJ, Sorajja P, Sundt TM, 3rd, Thomas JD, Members AATF. 2014 AHA/ACC Guideline for the Management of Patients With Valvular Heart Disease: a report of the American College of Cardiology/American Heart Association Task Force on Practice Guidelines. Circulation 2014;129(23):e521-643.

5. Peeters F, Meex SJR, Dweck MR, Aikawa E, Crijns H, Schurgers LJ, Kietselaer B. Calcific aortic valve stenosis: hard disease in the heart: $\mathrm{A}$ biomolecular approach towards diagnosis and treatment. Eur Heart J 2017.

6. Small A, Kiss D, Giri J, Anwaruddin S, Siddiqi H, Guerraty M, Chirinos JA, Ferrari G, Rader DJ. Biomarkers of Calcific Aortic Valve Disease. Arterioscler Thromb Vasc Biol 2017;37(4):623-632.

7. Fraser CG. Biological variation: from principles to practice. In. Washington DC: AACC Press; 2001.

8. Fraser CG. Reference change values. Clin Chem Lab Med 2011;50(5):807-12.

9. Bailey D, Bevilacqua V, Colantonio DA, Pasic MD, Perumal N, Chan MK, Adeli K. Pediatric within-day biological variation and quality specifications for 38 biochemical markers in the CALIPER cohort. Clin Chem 2014;60(3):518-29.

10. Harris EK. Effects of intra- and interindividual variation on the appropriate use of normal ranges. Clin Chem 1974;20(12):1535-42.

11. Frankenstein L, Wu AH, Hallermayer K, Wians FH, Jr., Giannitsis E, Katus HA. Biological variation and reference change value of high-sensitivity troponin $\mathrm{T}$ in healthy individuals during short and intermediate follow-up periods. Clin Chem 2011;57(7):1068-71.

12. Klinkenberg LJ, van Dijk JW, Tan FE, van Loon LJ, van Dieijen-Visser MP, Meex SJ. Circulating cardiac troponin T exhibits a diurnal rhythm. J Am Coll Cardiol 2014;63(17):1788-95.

13. Meijers WC, van der Velde AR, Muller Kobold AC, Dijck-Brouwer J, Wu AH, Jaffe A, de Boer RA. Variability of biomarkers in patients with chronic heart failure and healthy controls. Eur J Heart Fail 2017;19(3):357-365.

14. van der Linden N, Hilderink JM, Cornelis T, Kimenai DM, Klinkenberg LJJ, van Doorn WP, Litjens EJR, van Suijlen JDE, van Loon LJC, Bekers O, Kooman JP, Meex SJR. Twenty-Four-Hour Biological Variation Profiles of Cardiac Troponin I in Individuals with or without Chronic Kidney Disease. Clin Chem 2017;63(10):1655-1656.

15. Wu AH, Smith A. Biological variation of the natriuretic peptides and their role in monitoring patients with heart failure. Eur J Heart Fail 2004;6(3):355-8.

16. Wu AH, Wians F, Jaffe A. Biological variation of galectin-3 and soluble ST2 for chronic heart failure: implication on interpretation of test results. Am Heart J 2013;165(6):995-9.

17. Gardezi SK, Coffey S, Prendergast BD, Myerson SG. Serum biomarkers in valvular heart disease. Heart 2017. 
18. Otto CM, Burwash IG, Legget ME, Munt BI, Fujioka M, Healy NL, Kraft CD, Miyake-Hull CY, Schwaegler RG. Prospective study of asymptomatic valvular aortic stenosis. Clinical, echocardiographic, and exercise predictors of outcome. Circulation 1997;95(9):2262-70.

19. Rosenhek R, Binder T, Porenta G, Lang I, Christ G, Schemper M, Maurer G, Baumgartner H. Predictors of outcome in severe, asymptomatic aortic stenosis. N Engl J Med 2000;343(9):611-7.

20. Bartlett WA, Braga F, Carobene A, Coskun A, Prusa R, Fernandez-Calle P, Roraas T, Jonker N, Sandberg S, Biological Variation Working Group EFoCC, Laboratory M. A checklist for critical appraisal of studies of biological variation. Clin Chem Lab Med 2015;53(6):879-85.

21. Levey AS, Stevens LA, Schmid CH, Zhang YL, Castro AF, 3rd, Feldman HI, Kusek JW, Eggers P, Van Lente F, Greene T, Coresh J, Ckd EPI. A new equation to estimate glomerular filtration rate. Ann Intern Med 2009;150(9):604-12.

22. Evangelista A, Flachskampf F, Lancellotti P, Badano L, Aguilar R, Monaghan M, Zamorano J, Nihoyannopoulos P, European Association of E. European Association of Echocardiography recommendations for standardization of performance, digital storage and reporting of echocardiographic studies. Eur J Echocardiogr 2008;9(4):438-48.

23. Fraser CG, Harris EK. Generation and application of data on biological variation in clinical chemistry. Crit Rev Clin Lab Sci 1989;27(5):409-37.

24. IUPAC. Protocol for the design, conduct and interpretation of method-performance studies. Pure Appl Chem 1995;67(2):331-343.

25. Reed $A H$, Henry RJ, Mason WB. Influence of statistical method used on the resulting estimate of normal range. Clin Chem 1971;17(4):275-84.

26. Dixon WJ. Processing Data for Outliers. Biometrics 1953;9(1):74-89.

27. Roraas T, Petersen PH, Sandberg S. Confidence intervals and power calculations for withinperson biological variation: effect of analytical imprecision, number of replicates, number of samples, and number of individuals. Clin Chem 2012;58(9):1306-13.

28. Burdick RK, Graybill FA. Confidence intervals on variance components. New York: Marcel Dekker; 1992.

29. Harris EK, Yasaka T. On the calculation of a "reference change" for comparing two consecutive measurements. Clin Chem 1983;29(1):25-30.

30. Petersen PH, Fraser CG, Sandberg S, Goldschmidt H. The index of individuality is often a misinterpreted quantity characteristic. Clin Chem Lab Med 1999;37(6):655-61.

31. Fokkema MR, Herrmann Z, Muskiet FA, Moecks J. Reference change values for brain natriuretic peptides revisited. Clin Chem 2006;52(8):1602-3.

32. Henri C, Dulgheru R, Magne J, Caballero L, Laaraibi S, Davin L, Kou S, Voilliot D, Nchimi A, Oury C, Pierard LA, Lancellotti P. Impact of Serial B-Type Natriuretic Peptide Changes for Predicting Outcome in Asymptomatic Patients With Aortic Stenosis. Can J Cardiol 2016;32(2):183-9.

33. Lancellotti P, Dulgheru R, Magne J, Henri C, Servais L, Bouznad N, Ancion A, Martinez C, Davin L, Le Goff C, Nchimi A, Pierard L, Oury C. Elevated Plasma Soluble ST2 Is Associated with Heart Failure Symptoms and Outcome in Aortic Stenosis. PLoS One 2015;10(9):e0138940.

34. Rosjo H, Andreassen J, Edvardsen T, Omland T. Prognostic usefulness of circulating highsensitivity troponin $\mathrm{T}$ in aortic stenosis and relation to echocardiographic indexes of cardiac function and anatomy. Am J Cardiol 2011;108(1):88-91.

35. Shen M, Tastet L, Bergler-Klein J, Pibarot P, Clavel MA. Blood, tissue and imaging biomarkers in calcific aortic valve stenosis: past, present and future. Curr Opin Cardiol 2018;33(2):125-133.

36. Schindler EI, Szymanski JJ, Hock KG, Geltman EM, Scott MG. Short- and Long-term Biologic Variability of Galectin-3 and Other Cardiac Biomarkers in Patients with Stable Heart Failure and Healthy Adults. Clin Chem 2016;62(2):360-6.

37. Harris EK. Statistical aspects of reference values in clinical pathology. Prog Clin Pathol 1981;8:45-66. 


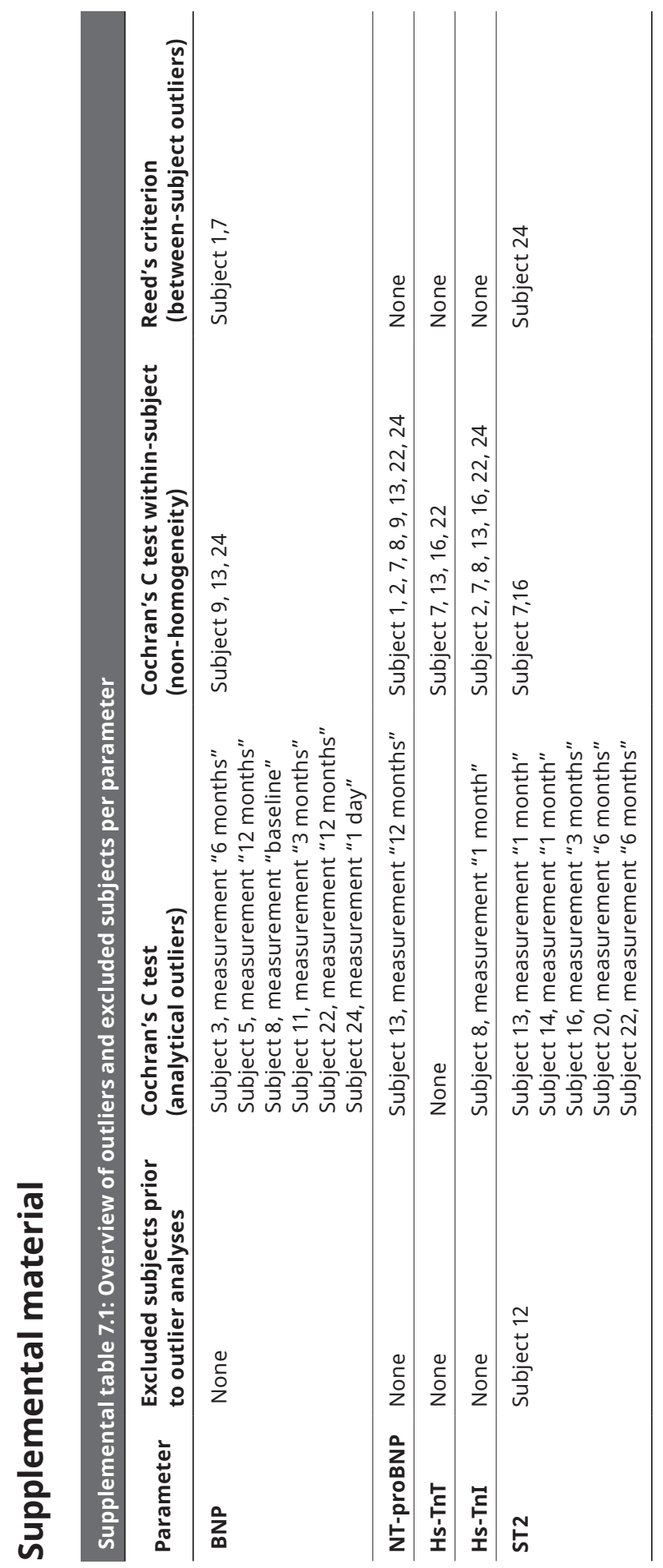




\section{Chapter 8}




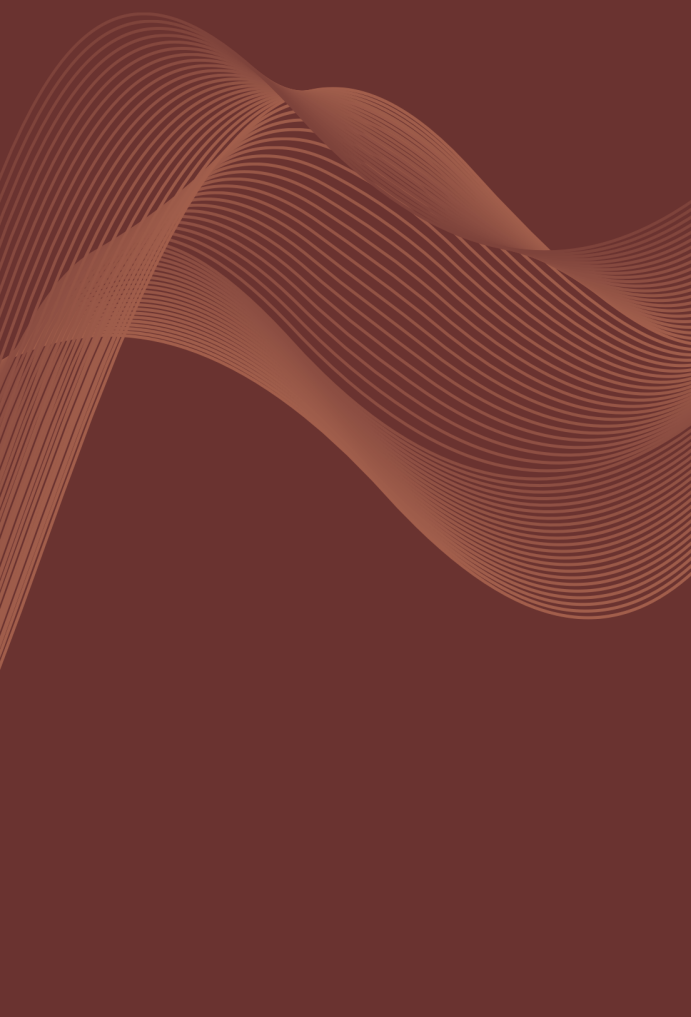

\section{Labtracker, a medical smartphone app for the interpretation of consecutive laboratory results: an external validation study}

J.M. Hilderink, R.J.M.W. Rennenberg, F.H.M. Vanmolkot, O. Bekers, R.P. Koopmans, S.J.R. Meex 


\begin{abstract}
Objectives

When monitoring patients over time, clinicians may struggle to distinguish 'real changes' in consecutive blood parameters from so-called natural fluctuations. In practice, they have to do so by relying on their clinical experience and intuition. We developed Labtracker, a medical app that calculates the probability that an increase or decrease over time in a specific blood parameter is real, given the time between measurements.
\end{abstract}

\title{
Design
}

We presented patient cases to 135 participants to examine whether there is a difference between medical students, residents and experienced clinicians when it comes to interpreting changes between consecutive laboratory results. Participants were asked to interpret if changes in consecutive laboratory values were likely to be 'real or rather due to natural fluctuations. The answers of the study participants were compared to the calculated probabilities by the app Labtracker and the concordance rates were assessed.

\section{Setting and participants}

Medical students $(n=92)$, medical residents from the department of internal medicine $(n=19)$ and internists $(n=24)$ at a Dutch University Medical Centre.

\section{Primary and secondary outcome measures}

Concordance rates between the study participants and the calculated probabilities by the app Labtracker were compared. Besides, we tested whether physicians with clinical experience scored better concordance rates with the app Labtracker than inexperienced clinicians.

\section{Results}

Medical residents and internists showed significantly better concordance rates with the calculated probabilities by the app Labtracker than medical students, regarding their interpretation of differences between consecutive laboratory results $(p=0.009$ and $\mathrm{p}<0.001$, respectively).

\section{Conclusion}

The app Labtracker could serve as a clinical decision tool in the interpretation of consecutive laboratory test results, and could contribute to rapid recognition of parameter changes by physicians. 


\section{Background}

Medical doctors frequently monitor the laboratory results of patients to determine whether their condition is stable, improving or deteriorating, and to decide whether treatment should be continued, initiated, or rather be postponed. Hence, an intuitive 'reference frame' of the patient can be formed by repeated assessment of laboratory parameters. An expertise-based reference frame is often lacking in less experienced clinicians, such as medical residents, or medical students. Hence, it can be difficult for less experienced clinicians to distinguish natural fluctuations in laboratory results from 'real changes' and vice versa.

According to a study of Jones et al., 1 in 5 medical graduates identified themselves as being 'less than competent' in using laboratory testing (1). Additionally, a study of Hickner et al. demonstrated that even experienced clinicians reported uncertainty in interpreting laboratory test results in approximately $10 \%$ of the ordered laboratory tests (2).

To help (inexperienced) clinicians to make the distinction whether a change between measurements is real or not, we developed a medical app (Labtracker),that calculates the probability of a 'real change' between two consecutive laboratory results. It is simple to use in clinical practice and provides evidence-based decision support to complement intuitive interpretation of changes in consecutive laboratory results.

The current study tests the hypothesis that experienced clinicians show better concordance rates with Labtracker-based calculations than medical students and less experienced clinicians. 

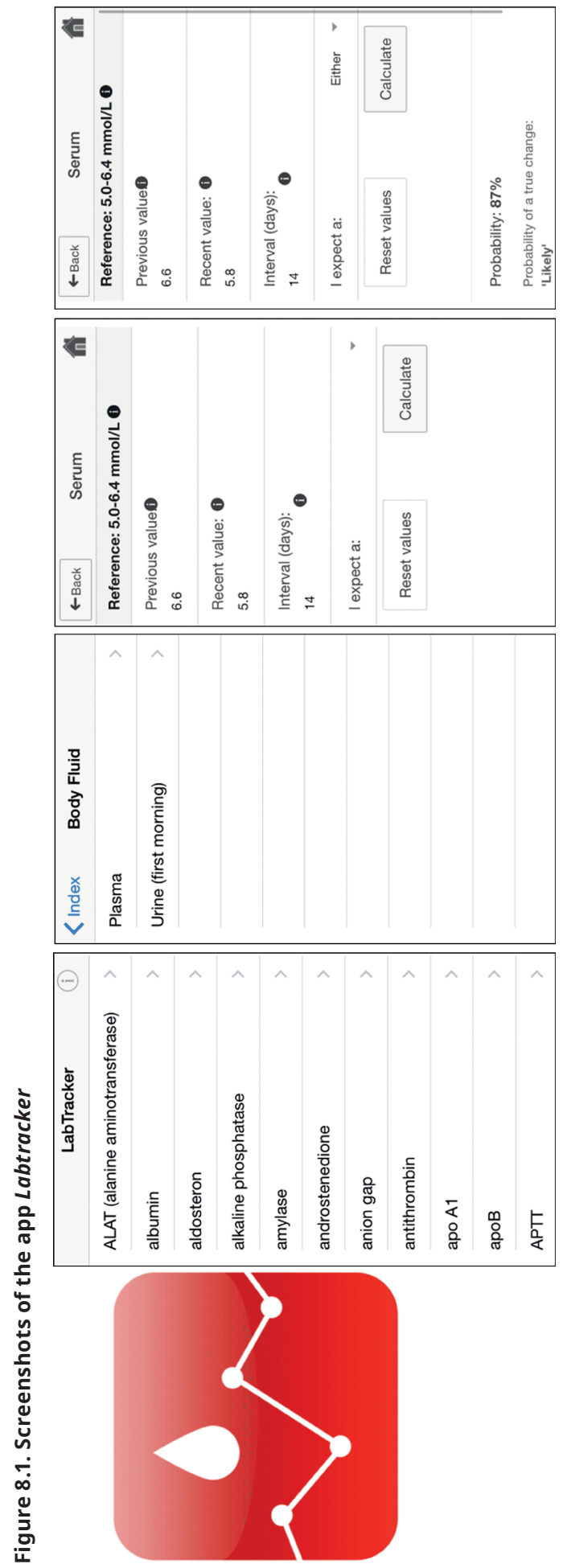


\section{Methods}

This study was approved by the Dutch Association for Medical Education (NVMO). Study participants provided written informed consent.

\section{Study participants}

135 participants were included: 92 medical students, 19 medical residents, and 24 medical specialists. We scheduled six days on which medical students participated in our study. All students were at the same stage of their internship and $92 \%$ of them were in their first medical internship (usually in their fourth year of the six years training to become a medical doctor in the Netherlands). The medical residents and medical specialists, all employed at the department of internal medicine at Maastricht University Medical Centre, were recruited during the daily morning briefing. The mean number of clinical experience years was $24 \pm 9$ for the internists and $3.5 \pm 2$ for the residents.

Exclusion criteria were: medical students in the bachelor phase of their studies and residents or specialists of a specialty other than internal medicine.

\section{Study design}

Ten fictive clinical cases were presented to the study participants. These cases were conceived and developed by the authors of this article. Central to each case was to interpret the likelihood of change between consecutive measurements of a certain laboratory parameter. The time interval between the measurements varied for all cases. The cases can be found in the supplemental material.

The participants were asked to categorize the presented changes in one of four likelihoods: 'unlikely' (<50\%), 'doubtful' (50-80\%), 'probably' (80-95\%), and 'very likely' (> 95\%). Participants answered these questions individually. The extent to which the responses of the participants were in accordance with the calculated probabilities by the app Labtracker was analyzed.

The laboratory parameters that were incorporated in the cases were: hemoglobin, leukocytes, thyroid stimulating hormone (TSH), vitamin B12, alanine aminotransferase (ALAT), glucose, glycosylated hemoglobin type A1c (HbA1c), creatinine, activated partial thromboplastin time ( $\mathrm{PPTT}$ ) and C-reactive protein (CRP). 


\section{Statistical analysis}

All answers given by the study participants were compared to the calculated probabilities by the app Labtracker. Two statistical analyses were performed. Firstly, differences among the study groups were calculated using one-way analysis of variance (ANOVA). P-values < 0.05 were considered statistically significant. Comparisons between pairs of groups were adjusted for multiple testing using the Bonferroni correction.

Secondly, two groups were compared; the medical students formed the 'inexperienced group' and the medical residents and internists together formed the 'experienced group'. The two groups were compared using the Fisher's exact test. All statistical calculations were performed using SPSS version 23 (IBM SPSS Statistics. IBM Corporation, Armonk, New York).

\section{Calculating the probability of change using the app Labtracker}

For a change between consecutive measurements to become significant, the difference must be larger than the change that would reasonably be expected due to normal analytical- and biological variation. This is termed the reference change value (RCV) and was first described by Fraser et al (3). The RCV has become an established concept in laboratory medicine.

The RCV differs for each parameter and depends on the within-subject biological variation $\left(\mathrm{CV}_{\mathrm{I}}\right)$ and the analytical variation $\left(\mathrm{CV}_{\mathrm{A}}\right)$ and can be calculated as follows $(4,5)$ :

$$
R C V=\sqrt{2} * Z * \sqrt{C V_{A}^{2}+C V_{I}^{2}}
$$

In this formula, the Z-score represents the number of standard deviations and corresponds to the desired probability. Commonly used Z-scores are 1.96 and 2.56. These Z-scores calculate the percentage increase or decrease that is required to become statistically significant, with a false positive rate of $5 \%,(p<0.05)$ and $1 \%$ $(p<0.01)$, respectively.

An important side note to calculate the RCV is that not all clinical decisions are made with statistical probabilities of $>95 \%$. In other words, some changes are strictly not statistically significant, but the probability may be high enough to lead to a treatment or clinical decision. For example, a hemoglobin concentration of 6.1 
$\mathrm{mmol} / \mathrm{L}$ was measured in a male patient. After a week, it dropped to $5.8 \mathrm{mmol} / \mathrm{L}$. Although the likelihood probability of a 'real' decrease in hemoglobin level is less than $95 \%$ in this case - namely around $70 \%$ - the clinician may still decide to use additional diagnostic testing or to treat the patient.

For implementation in the smartphone app, we rearranged the RCV-formula so that the Z-score (and therefore the likelihood probability) is the unknown. The probability of a real change between consecutive measurements will then be calculated. The rearranged RCV-formula is:

$$
\text { Z-score }=\frac{\text { percentage change between consecutive measurements }}{\sqrt{2 *\left(C V_{A}^{2}+C V_{I}^{2}\right)}}
$$

The calculations of the app Labtracker are based on the above formula. This makes it possible to compare two consecutive laboratory results and to calculate the probability of a real change.

\section{Time-dependency of within-subject biological variation in some parameters}

In a number of laboratory parameters, the within-subject biological variation $\left(\mathrm{CV}_{\mathrm{I}}\right)$ changes over time. We systematically reviewed published biological variation studies of 106 laboratory tests and found a time-dependent increase of the biological variation for 21 parameters. For the laboratory parameters with sufficient information about their biological variation in the scientific literature, the associations between biological variation data and the time interval to which they apply were modeled statistically and integrated into Labtracker. This enabled the calculation of probabilities of a change between measurements, while accounting for the time-span between consecutive measurements.

\section{Practical functionality of the app Labtracker}

In total, 106 parameters are available in Labtracker. When using the app, users can choose the desired parameter out of a list that is displayed in alphabetical order. Subsequently, they have to choose the 'body liquid' in which the measurements were performed and, if relevant, whether the blood sampling regards a male or a female person. However, this question is asked only to display an indication of the reference values, but it does not affect the final calculation of the probability. Then the user fills in the previous and the recent value, as well as the time interval in days between the measurements. Also the 'a priori expectation' of the change between 
the measurements has to be chosen ('rise' for an expected increase, 'fall' for an expected decrease or 'either' when there is no prior expectation). Labtracker will then calculate the probability that the change between the two measured values is a 'real change'. In figure 8.1, screenshots of the app were presented to visualize the functionality of the app.

Figure 8.2. Percentage concordant answers per group

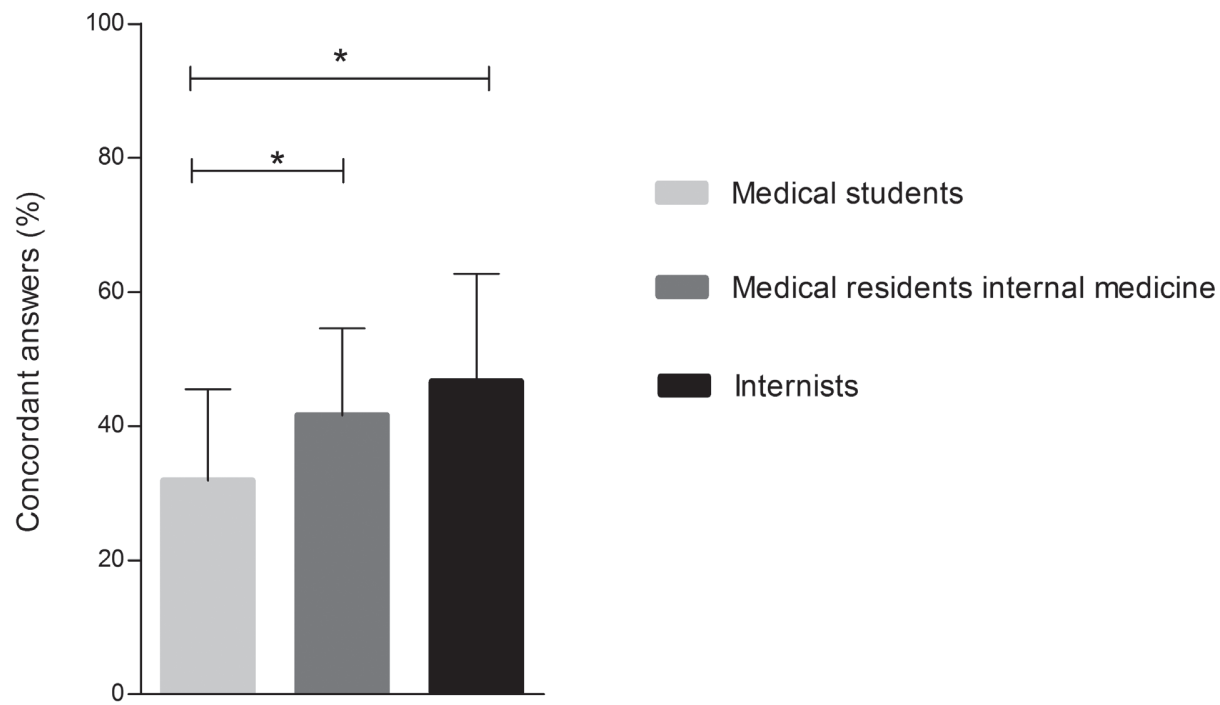

Values are mean percentages \pm SD.

Medical students vs. internists, $\mathrm{p}<0.001 ; 95 \% \mathrm{CI} 6.96-22.59$, medical students vs. medical residents, $\mathrm{p}=0.009 ; 95 \%$ CI 1.10-18.28, medical residents vs. internists, $\mathrm{p}=0.321 ; 95 \%$ CI $-5.36-15.53$. $p$-values $<0.05$ were considered statistically significant $(*)$.

\section{Results}

Data collection was incomplete for two subjects (1.5\%); it concerns two medical students who did not fill in all questions and therefore, they were excluded from all analyses.

\section{Psychometric analysis}

We performed the 'Item Test Correlation (RIT)' test, a psychometric analysis to check the distinctiveness of the case questions $(6,7)$. Briefly, the RIT is a correlation coefficient, which can vary between -1 and 1; a question with a RIT score of 0 does not distinguish between good and poor performers. If the RIT score is negative then the question may not be valid and should be removed $(6,7)$. The RIT score of 
the aPTT-question had a negative value and therefore, this question was excluded from further analyses. The RIT score of the other questions were all considered sufficient.

Figure 8.3. Percentage concordant answers when also the probability adjacent to the designated category was considered correct.

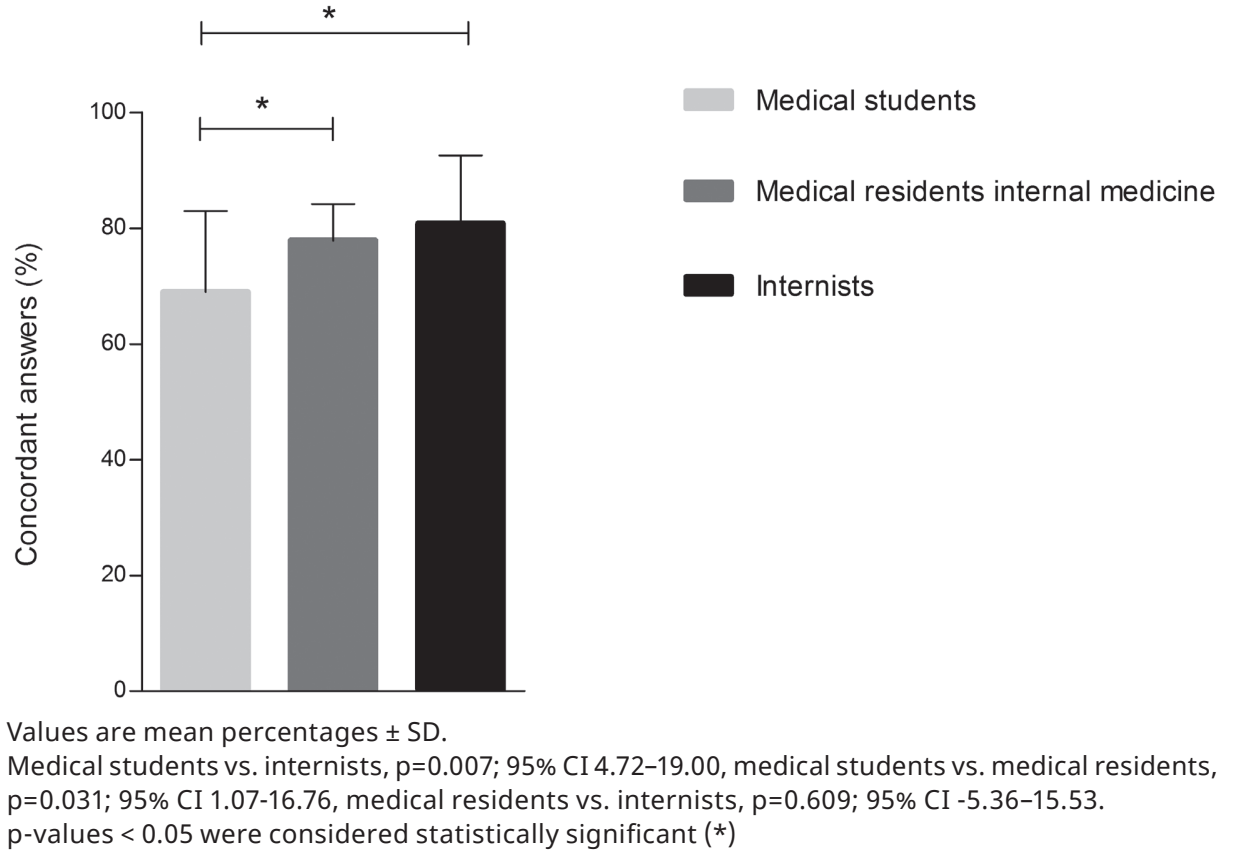

Comparison of the app Labtracker with the estimates of physicians

The correct answer was defined as the answer that matched exactly with the calculated probability by the app Labtracker i.e. the answer category containing the calculated probability percentage.

In figure 8.2 , the estimates of the study groups were shown. Participants with extensive clinical experience showed higher concordance rates with Labtrackercalculations; with internists and the medical residents scoring significantly better than the medical students ( $p<0.001$ and $p=0.021$, respectively). The percentage of questions answered correctly by the medical residents did not differ significantly from the internists ( $p=0.719 ; 95 \%$ CI $-5.4-15.5)$. 
A sensitivity analysis was conducted that allowed a broader range to define concordant answers. In this sensitivity analysis, not only the answer category that matched exactly with the calculated probability by Labtracker was considered correct, but also the answer category adjacent to that category. The results of this analysis are shown in figure 8.3. On average, the medical students answered 69\% of the questions correctly, the medical residents $78 \%$ and the internists $81 \%$. Similar to the primary analysis, concordance rates of answers from internists with the Labtracker-calculated probabilities were significantly better than medical students ( $p<0.001 ; 95 \%$ CI 4.7 - 19.0). Again, the difference between medical residents and medical students was statistically significant as well ( $p=0.020 ; 95 \%$ CI $1.1-16.8$ ). And, like the first analysis, concordance rates between the medical residents and Labtracker did not differ significantly from the internists ( $p=1.000 ; 95 \%$ CI $-12.5-6.6$ ). Therefore, in subsequent analyses, internists and medical residents were pooled as one group (the experienced clinicians) and compared with medical students.

\section{Analysis per parameter}

The scores of the study participants for each parameter were assessed separately. Figure 8.4 depicts the percentage of correct answers from the study groups per parameter. The 'experienced' group (the residents and the internists) was compared with the medical students. In general, the experienced group demonstrated the most concordant answers, compared to the inexperienced medical students. The experienced clinicians scored best on the parameter hemoglobin (95\%) and the parameter they scored the worst on was creatinine (28\%). The medical students scored best on the hemoglobin parameter as well (82\%). They scored worst on the TSH question (6\%). 


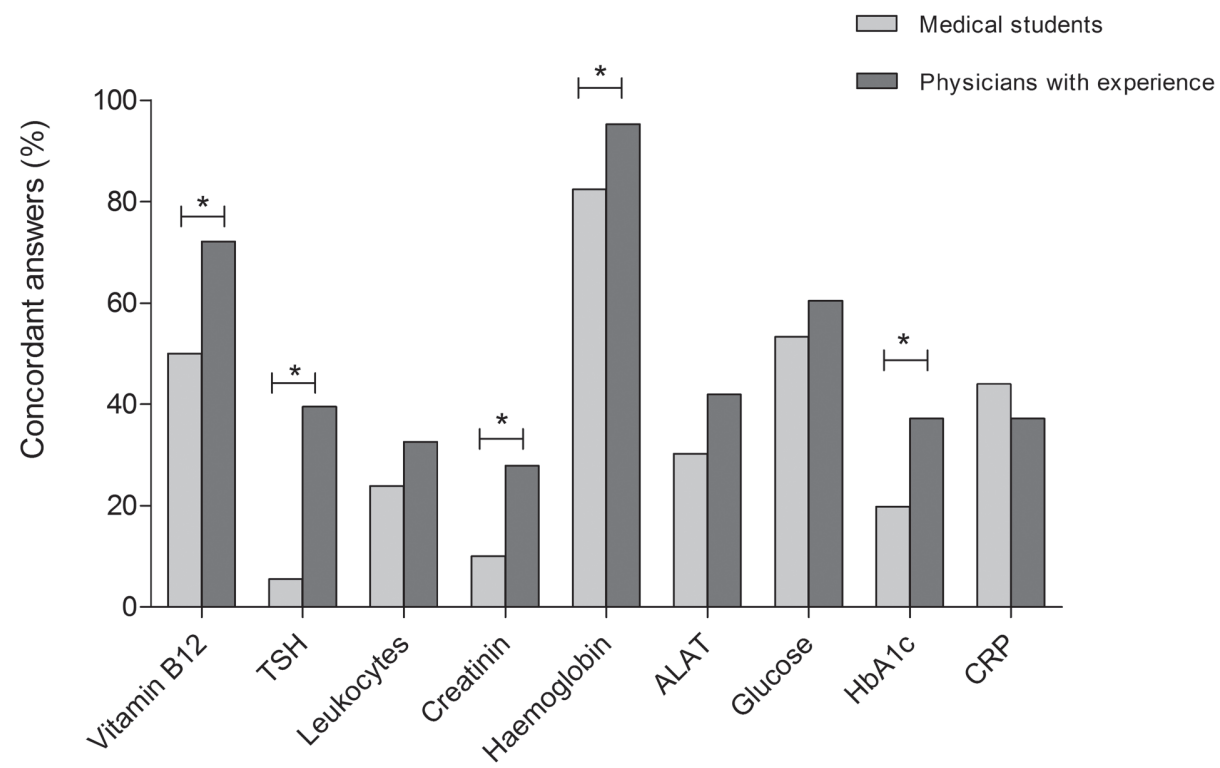

TSH $p<0.001$, creatinine $p=0.012$, vitamin B12 $p=0.024, \mathrm{HbA} 1 \mathrm{c} p=0.036$, haemoglobin $p=0.056$, ALAT $p=0.237$, leukocytes $p=0.307$, glucose $p=0.462$, CRP $p=0.574$

In this figure, the medical residents and the internists were combined into the group 'clinicians with experience'

In accordance with the previous analysis, we conducted a sensitivity analysis in which the category adjacent to the correct answer was also considered to be correct. These results are shown in figure 8.5. Again, the experienced clinician gave the most concordant answers. In this analysis, the experienced group (residents and internists) scored $88 \%$ correct answers on average. They scored the best on the vitamin B12, leukocytes, and hemoglobin questions (98\%). The TSH question was the least well answered of all parameters (70\%). The medical students scored $90 \%$ correct answers on the hemoglobin question. The least concordant question by the students was TSH (44\%). 


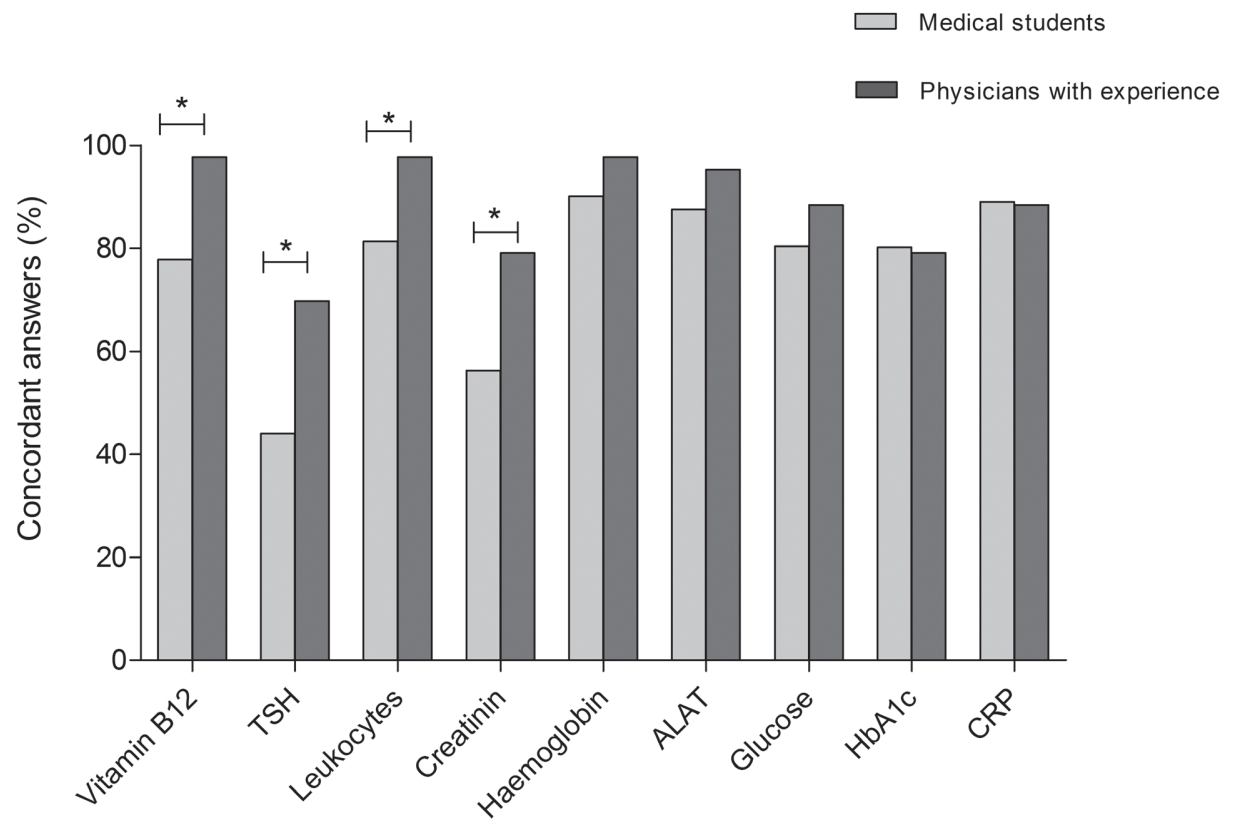

Vitamin B12 $p=0.002$, TSH $p=0.006$, leukocytes $p=0.012$, hemoglobin $p=0.167$, creatinine $p=0.012$, ALAT $p=0.221$, glucose $p=0.329$, HbA1c $p=1.000$, CRP $p=1.000$.

In this figure, the medical residents and the internists were combined into the group 'clinicians with experience

\section{Discussion}

The current study demonstrated that experienced clinicians show better concordance rates with the calculated probabilities by the app Labtracker than less experienced medical students. Stratified analyses reveal that this pattern holds true for the majority of studied laboratory parameters. Concordance rates with the app demonstrate an incremental improvement from medical students, to residents and internists. The differences between clinicians with experience (internists and residents) and inexperienced medical students were statistically significant. This outcome illustrates the relevance and added value of the medical app Labtracker.

The clear association between the degree of clinical experience and improved concordance rates with the calculated probabilities by the app supports the validity of Labtracker calculations. Improved concordance rates with increasing clinical 
experience suggest that Labtracker may well be of benefit for less experienced clinicians, such as medical students.

However, Labtracker is not only a useful tool for inexperienced clinicians. We believe the app can form a 'bridge' between laboratory science and clinical practice. Labtracker could be useful in reducing information overload and enabling rapid recognition of parameter changes by expert clinicians, especially in time constrained and rapidly changing environments (e.g., intensive care units). Clinicians do not have to make cumbersome calculations when estimating whether a change between consecutive measurements is a real change or not. By using the app, the quality of the laboratory information that often plays an important role in clinical decisions will improve. Labtracker enables clinicians to make efficient and standardized interpretations with minimal cognitive effort. Of course, the application will never replace clinical judgment, but it can serve as a supportive aid for diagnosis and treatment.

In addition, in current clinical practice, some clinical tasks that were always performed by physicians now more and more become the responsibility of physician assistants or nurse specialists. Such 'shifts of tasks' can be for instance, a diabetic nurse who monitors the routine diabetic check-ups. In 2014, LentersWestra et al. demonstrated that there are significant differences in interpretation of changes in $\mathrm{HbA1c}$ results between physicians and nurses (8). Labtracker may serve as a useful support to standardize care in such a situation.

The benefit offered by the app may be parameter dependent. According to this external validation study, the concordance rates with the calculated probabilities varied from $6 \%$ for students for the TSH question to $98 \%$ for experienced clinicians for the hemoglobin question. This difference in concordance rates suggests that the 'number intuition' of a clinician differs per laboratory parameter and varies with clinical experience. It may be difficult for a clinician to estimate how a 'normal value' of a parameter fluctuates over time in an individual if the clinician has little actual experience with that parameter. In other words, a clinician with more experience in interpreting a specific parameter can better estimate its biological variation. Since the scores of the medical residents were not significantly different from those of the internists, it might be assumed that medical residents develop their 'number intuition' early in the residency training. 
The participants scored not all parameters equally concordant. A potential explanation for the 'worse scored questions' could be that not all laboratory parameters are widely used in clinical practice, and that clinicians therefore have little experience in interpreting those parameters. Another potential explanation could be that not all questions were of equal difficulty.

Some limitations in the current study merit attention. First, the groups of medical residents and specialists were relatively small ( $n=19$ and $n=24$, respectively). Second, only nine parameters were tested, with one single interpretation per parameter. However, our estimation is that the study groups are a good reflection of the total population of people in these groups. In addition, the parameters we studied are commonly used in clinical practice and cover a broad range of clinical domains. Therefore, it can reasonably be expected that the results of this study can be generalized to all commonly used laboratory parameters. Third, the cases were not presented in random order to the subjects. It is possible that the subjects' performances were partially influenced by the order of case presentation. However, if there were a learning curve, then this should be the same for all study groups.

Fourth, it remains unclear whether the use of the medical app Labtracker, would affect clinical decision-making. In that respect, a study that incorporated medical decision-making, based on hypothetical cases involving laboratory test result interpretation(s), would be an interesting addition. The hypothesis that could then be tested is whether inexperienced clinicians make the same decisions as more experienced clinicians when they use the app Labtracker.

In 2014, Abbot et al. demonstrated that the self-assessed knowledge about interpreting laboratory results of inexperienced medical residents significantly improved after receiving an educational program about 'the basics of laboratory test ordering and interpretation (9). This finding endorses the importance of education for inexperienced clinicians. Elaborating on this, it would be interesting to investigate whether Labtracker, as an educative aid, could provide better medical decision-making.

In conclusion, the current study has provided insight into the intuition of medical students with hardly any clinical experience, clinicians with some experience (medical residents from the department of internal medicine), and experienced clinicians (internists), when interpreting a difference between consecutive 
laboratory results The findings suggest the existence of a trend that the 'number intuition' of a clinician improves with more years of experience. The development of this intuition fosters a better interpretation of laboratory results and could possible improve clinical decision-making. In that sense, the use of the medical app Labtracker can be a useful tool, especially for inexperienced clinicians. Besides, Labtracker could be useful in reducing information overload and enabling rapid recognition of parameter changes by expert clinicians. 


\section{References}

1. Jones A, McArdle PJ, O'Neill PA. Perceptions of how well graduates are prepared for the role of pre-registration house officer: a comparison of outcomes from a traditional and an integrated PBL curriculum. Med Educ 2002;36(1):16-25.

2. Hickner J, Thompson PJ, Wilkinson T, et al. Primary care physicians' challenges in ordering clinical laboratory tests and interpreting results. J Am Board Fam Med 2014;27(2):268-74.

3. Fraser CG. Reference change values. Clin Chem Lab Med 2012;50(5):807-12.

4. Ricos C, Alvarez V, Perich C, et al. Rationale for using data on biological variation. Clin Chem Lab Med 2015;53(6):863-70.

5. Ricos C, Alvarez V, Cava F, et al. Current databases on biological variation: pros, cons and progress. Scand J Clin Lab Invest 1999;59(7):491-500.

6. Ebel RL, Frisbie DA. Essentials of Educational Measurement. Pearson, 5th edition 1991.

7. Ory JC, Ryan KE. Tips for Improving Testing and Grading. Sage Publications Inc 1993.

8. Lenters-Westra E, Schindhelm RK, Bilo HJ, et al. Differences in interpretation of haemoglobin A1c values among diabetes care professionals. Neth J Med 2014;72(9):462-6.

9. Abbott $M$, Paulin $H$, Sidhu $D$, et al. Laboratory tests, interpretation, and use of resources: a program to introduce the basics. Can Fam Physician 2014;60(3):e167-72. 


\section{Supplementary material: Case questions}

Correct answers, calculated by the app Labtracker, are shown in bold.

Reference values are from Maastricht University Medical Center (MUMC).

1. Glucose

Reference values fasting glucose [4.0 - $7.0 \mathrm{mmol} / \mathrm{L}]$

First measurement: [7.3 $\mathrm{mmol} / \mathrm{L}]$

Second measurement (two weeks later) : [6.9 $\mathrm{mmol} / \mathrm{L}]$

What is the probability likelihood that this is a real change?

A: Unlikely ( $<50 \%)$

B: Doubtful (50-80\%)

C: Likely (80-95\%)

D: Very likely(>95\%)

2. TSH

Reference values TSH [0.4-4.3 $\mathrm{mU} / \mathrm{L}]$

First measurement: [6.5 mU/L]

Second measurement (three months later) : [7.1 mU/L]

What is the probability likelihood that this is a real change?
A: Unlikely $(<50 \%)$
B: Doubtful (50-80\%)
C: Likely (80-95\%)
D: Very likely (>95\%) 
3. CRP

Reference values CRP: $<10 \mathrm{mg} / \mathrm{L}$

First measurement:

[79 mg/L]

Second measurement (two days later):

[106 mg/L]

What is the probability likelihood that this is a real change?
A: Unlikely $(<50 \%)$
B: Doubtful (50-80\%)
C: Likely (80-95\%)
D: Very likely $(>95 \%)$

\section{Hemoglobin}

\section{Reference values hemoglobin for a woman (7.5-9.5 $\mathrm{mmol} / \mathrm{L})$}

First measurement:

[7.0 $\mathrm{mmol} / \mathrm{L}]$

Second measurement (six months later):

[7.2 $\mathrm{mmol} / \mathrm{L}]$

What is the probability likelihood that this is a real change?
A: Unlikely $(<50 \%)$
B: Doubtful (50-80\%)
C: Likely (80-95\%)
D: Very likely (>95\%)

5. HbA1c

\section{Target value $\mathrm{HbA1c}<58 \mathrm{mmol} / \mathrm{mol}$}

First measurement:

[55 $\mathrm{mmol} / \mathrm{mol}](=7.2 \%)$

Second measurement ( 6 months later): $\quad[60 \mathrm{mmol} / \mathrm{mol}](=\mathbf{7 . 6 \%})$

What is the probability likelihood that this is a real change?
A: Unlikely $(<50 \%)$
B: Doubtful (50-80\%)
C: Likely (80-95\%)
D: Very likely (>95\%) 


\section{Leukocytes}

\section{Reference values leukocytes [3.5-11.0*10\%/L]}

First measurement:

[12*10\%/L]

Second measurement (14 days later):

What is the probability likelihood that this is a real change?
A: Unlikely $(<50 \%)$
B: Doubtful (50-80\%)
C: Likely (80-95\%)
D: Very likely (>95\%)

7. aPTT (this question was excluded from analyses)

\section{Reference values aPTT [18-36 sec]}

First measurement: [30 sec]

Second measurement (a few hours later) : [34 sec]

What is the probability likelihood that this is a real change?
A: Unlikely $(<50 \%)$
B: Doubtful (50-80\%)
C: Likely (80-95\%)
D: Very likely (>95\%)

\section{Vitamin B12}

\section{Reference values vitamin B12 [250-850 pmol/L]}

First measurement:

Second measurement (3 months later): [125 pmol/L]

What is the probability likelihood that this is a real change?
A: Unlikely ( $<50 \%)$
B: Doubtful (50-80\%)
C: Likely (80-95\%)
D: Very likely (>95\%) 


\section{ALAT}

Reference values ALAT $<45 U / L$

First measurement:

[44 U/L]

Second measurement ( 6 months later): [ [55 U/L]

What is the probability likelihood that this is a real change?
A: Unlikely $(<50 \%)$
B: Doubtful (50-80\%)
C: Likely (80-95\%)
D: Very likely (>95\%)

10. Creatinine
Reference values creatinine for men [60-110 $\mu \mathrm{mol} / \mathrm{L}]$
First measurement:
[89 $\mu \mathrm{mol} / \mathrm{L}]$
Second measurement (one year later): [109 $\mu \mathrm{mol} / \mathrm{L}]$
What is the probability likelihood that this is a real change?
A: Unlikely $(<50 \%)$
B: Doubtful (50-80\%)
C: Likely (80-95\%)
D: Very likely (>95\%) 


\section{Chapter 9}




\section{General discussion}


172 Chapter 9 
This thesis connects basic principles of laboratory medicine with daily clinical practice. Historically there is a strong intertwining between the hospital laboratory and various clinical departments (1-4). However, the theoretical concept of biological variation in relation to serial measurements, and especially its potential value in clinical decision-making, is relatively unknown to many physicians $(5,6)$. Monitoring patients over time and the repeated measurement of various biomarkers is common practice for almost all hospitalized patients, and frequently applied to ambulant patients with chronic diseases $(7,8)$. Changes between measurements can reflect clinical improvement or deterioration, and occur as a result of random fluctuation without clinical consequences. Clinicians rely on their intuition and experience to discriminate between these two possibilities. In this thesis biological variation data of biomarkers within subjects is used to develop an evidence-based approach for the monitoring of lab results over time. Within-subject biological variation is for most parameters the dominant source of variation, which defines whether observed changes between serial measurements should be ascribed to either "random fluctuation" or "real changes"(9-13).

This thesis addresses 4 major topics.

Chapter 2 describes the development of a medical smartphone app Labtracker. Labtracker uses biological variation data from peer-reviewed literature and novel studies published in this thesis to provide evidence based support for the interpretation of serial laboratory results. Hence it can complement clinical intuition of physicians to make clinical decisions, and serve as an educational tool for medical students and residents. Chapter 3, 4, 5, 6 and 7 are biological variation studies that extend the availability of peer-reviewed literature of biological variation data, with a focus on within-day biological variation. Chapters 4-7 compare biological variation in healthy subjects to those with various chronic diseases. Finally, in chapter 8, a validation study of Labtracker is reported.

In this discussion we will elaborate on these 4 topics and provide directions for further research. 


\section{Development of the medical smartphone app Labtracker}

The backbone of this thesis is the development of the medical smartphone app Labtracker (chapter 2). The app Labtracker interprets the magnitude of change between two consecutive laboratory results, while taking into account the biological variation of the observed parameter, the time interval between the measurements, and the analytical variation. It calculates the probability (from $0 \%-100 \%$ ) that the observed change is a real change. This output provides an individualized and objective assessment of the likelihood of a true change, and may benefit clinical decisions related to patient monitoring e.g. starting or changing a treatment.

Currently, physicians rely exclusively on their intuition and clinical experience when interpreting changes in consecutive laboratory results $(14,15)$. However, physicians often overestimated the analytical accuracy of biomarkers and presence of biological variation is not always appreciated (16-18). Especially for less trained or less experienced medical professionals, the lack of an easy reference for the interpretation of consecutive laboratory results can be challenging and may contribute to undesirable heterogeneity in clinical actions following biomarker measurements. Labtracker addresses this clinical problem by standardizing the interpretation of laboratory test results and hence contribute to more standardized care. Physicians, medical students, nurses and medical professionals may benefit from using this application, especially when their experience with the interpretation of changes between consecutive laboratory measurements is limited, or when using it for biomarkers they are less familiar with. A typical example of inconsistent interpretation among health care professionals is that of serially measured Hemoglobin A1c values. In a survey with various diabetes care professionals -104 physicians, 177 diabetes specialist nurses, and 248 primary care nurses- 96\% of physicians were aware of some inherent uncertainty when comparing serial Hba1C results, but only $56 \%$ of the nurses recognized some degree of random fluctuation between measurements as an intrinsic characteristic of biomarkers(19, 20). As a consequence, many nurses considered adjusting therapy based on very small, insignificant changes in $\mathrm{HbA} 1 \mathrm{c}$, whereas physicians adhered better to more clinically meaningful thresholds (20). This is just one of many clinical scenarios where Labtracker could contribute to better standardization, and prevent undue adjustments in the treatment of patients, based on incorrectly perceived biomarker changes. 


\section{Biological variation}

The term biological variation can either refer to between-subject biological variation $\left(\mathrm{CV}_{\mathrm{G}}\right)$ or to within-subject biological variation $\left(\mathrm{CV}_{\mathrm{I}}\right)(21,22)$. Both are fundamentally different and have different clinical applications. $\mathrm{CV}_{\mathrm{G}}$ can be estimated from a single biomarker measurement in a large group of subjects (typically 100-1000), and it is based on population reference intervals. Reference intervals are a very familiar concept for most physicians (23). In contrast, $\mathrm{CV}_{\mathrm{I}}$ is derived from repeated measurements in a small set of subjects (typically 10-20), and the calculated variability within subjects can be used for monitoring patients, e.g by calculating reference change values or from estimations by Labtracker (24). The ratio between the within-subject biological variation and the between-subject biological variation is termed "index of individuality" (25-27). The low index of individuality has important consequences for the use of conventional reference values and the interpretation of laboratory values. When the index of individuality of a biomarker is low (usually $<0.6$ ), the dispersion of values of a person will span only a small part of the reference interval, and reference intervals will have limited utility (21, $28,29)$. Hence, for these biomarkers, calculation of reference change values is particularly useful $(30,31)$. Reliable $\mathrm{CV}_{\mathrm{I}}$ data are indispensable for this purpose, and chapters 3-6 provide a reference data set that extends our understanding of within-subject biological variation, to be used for RCV calculations, with emphasis on the variation within 24 hours. Within-day $\mathrm{CV}_{\mathrm{I}}$ is useful in hospitalized patients with short time intervals between biomarker measurements (32). Data from our laboratory information system show that at least $10 \%$ of hospitalized patients have multiple serial measurements within 24 hours, a time window that has conceivably different variability than intervals of multiple days, weeks or months.

\section{Biological variation in healthy vs. non-healthy subjects}

A largely unverified assumption for the validity of using reference change values (or Labtracker) in clinical practice is that within-subject biological variation does not change substantially in various chronic diseases, compared to healthy subjects $(21,33)$. In fact, consistent biological variation in health and chronic disease is an important prerequisite for the use of reference change values and derived medical devices such as Labtracker. An individual's homeostatic set-point of a biomarker may increase or decrease in some chronic diseases, but variability around the setpoint is assumed to remain relatively constant. We tested this assumption for a number of biomarkers in by comparing $\mathrm{CV}_{\mathrm{I}}$ 's from CKD patients and aortic valve stenosis with healthy subjects (chapters 4, 5, 6 and 7, respectively), and did not 
identify biological variation shifts of a magnitude that would invalidate the use of RCV's, calculated with CVI data from healthy subjects, in these patients.

\section{Clinical interpretation and medical practice and limitations}

Fourth and final major topic of this thesis is the external validation of the smartphone app Labtracker (chapter 8). We evaluated concordance rates of Labtracker calculations with medical students, medical residents, and experienced physicians and showed progressively better concordance rates with Labtracker with increasing clinical experience. This confirms that especially less experienced medical professionals may benefit from using Labtracker. For this group, but likely also for nurses, physician assistants and other health care professionals, Labtracker could be useful in reducing information overload and allow more standardized recognition of parameter changes, and more aligned interpretation with the expert professional.

A limitation of Labtracker may be the conscious misinterpretation of 1-p, and translation of this value into a probability by Labtracker (34). Frequentist statistics is commonly misinterpreted in medicine, and also in the setting of Labtracker, we acknowledge that a true probability calculation would require a Bayesian approach, including the estimation of a prior probability of a change between serial measurements (35-37). Nevertheless, while being fully aware of this statistical nuance, we argue that the development of Labtracker, which can be easily understood and applied and provide clinical benefit to medical professionals, outweighs the limitation of statistical imperfection.

\section{Concluding remarks and future perspectives}

This thesis linked laboratory sciences to medical practice through the development of the medical smartphone app Labtracker and through extending the within-day biological variation database.

We propose a number of future directions. The first strategy would be to secure and extend the connection that Labtracker initiated, by incorporating the methodology and algorithms of Labtracker into the medical laboratory system of hospitals. Such infrastructure would also facilitate a particularly interesting future study: to evaluate whether Labtracker could not only harmonize interpretation, and clinical decision-making. 
The second future perspective relates to the use of more than one parameter at the same time, as well as calculations with three or more serial measurements. In clinical practice, most of the laboratory parameters are determined as a part of a set of laboratory parameters, and it is conceivable that combining these data will provide even more accurate estimations.

The third and final interesting future perspective is to extend the methodology behind Labtracker to commonly used non-laboratory measurements such as blood pressure, heart rate, oxygen saturation and temperature measurements. These measurements are performed multiple times per day in a large number of hospitalized patients. Provided that the analytical and within-subject biological variation of these determinations can be established, Labtracker could help harmonize their interpretation. 


\section{References}

1. Plebani M. Harmonization in laboratory medicine: More than clinical chemistry? Clin Chem Lab Med 2018;56:1579-86.

2. Plebani M, Graziani MS, Tate JR. Harmonization in laboratory medicine: Blowin' in the wind. Clin Chem Lab Med 2018;56:1559-62.

3. Pennestri F, Banfi G. Value-based healthcare: The role of laboratory medicine. Clin Chem Lab Med 2019;57:798-801.

4. Jackups R, Jr., Szymanski JJ, Persaud SP. Clinical decision support for hematology laboratory test utilization. Int J Lab Hematol 2017;39 Suppl 1:128-35.

5. Macklin SK, Jackson JL, Atwal PS, Hines SL. Physician interpretation of variants of uncertain significance. Fam Cancer 2019;18:121-6.

6. Loh TP, Metz MP. Indirect estimation of pediatric between-individual biological variation data for 22 common serum biochemistries. Am J Clin Pathol 2015;143:683-93.

7. van Engelen TSR, Wiersinga WJ, Scicluna BP, van der Poll T. Biomarkers in sepsis. Crit Care Clin 2018;34:139-52.

8. Fontana RJ, Lok AS. Noninvasive monitoring of patients with chronic hepatitis c. Hepatology 2002;36:S57-64.

9. Braga F, Panteghini M. Generation of data on within-subject biological variation in laboratory medicine: An update. Crit Rev Clin Lab Sci 2016;53:313-25.

10. Roraas T, Stove B, Petersen PH, Sandberg S. Biological variation: The effect of different distributions on estimated within-person variation and reference change values. Clin $\mathrm{Chem}$ 2016;62:725-36.

11. Roraas T, Stove B, Petersen PH, Sandberg S. Biological variation: Evaluation of methods for constructing confidence intervals for estimates of within-person biological variation for different distributions of the within-person effect. Clin Chim Acta 2017;468:166-73.

12. Coskun A, Braga F, Carobene A, Tejedor Ganduxe X, Aarsand AK, Fernandez-Calle P, et al. Systematic review and meta-analysis of within-subject and between-subject biological variation estimates of 20 haematological parameters. Clin Chem Lab Med 2019;58:25-32.

13. Coskun A, Carobene A, Kilercik M, Serteser M, Sandberg S, Aarsand AK, et al. Within-subject and between-subject biological variation estimates of 21 hematological parameters in 30 healthy subjects. Clin Chem Lab Med 2018;56:1309-18.

14. Vanstone M, Monteiro S, Colvin E, Norman G, Sherbino J, Sibbald M, et al. Experienced physician descriptions of intuition in clinical reasoning: A typology. Diagnosis (Berl) 2019;6:259-68.

15. Aldamiri KT, Alhusain FA, Almoamary A, Alshehri K, Al Jerian N. Clinical decision-making among emergency physicians: Experiential or rational? J Epidemiol Glob Health 2018;8:65-8.

16. Taylor TR. Clinical decision analysis. Methods Inf Med 1976;15:216-24.

17. Bear R, Schneiderman J. Decision analysis in clinical medicine. Can Med Assoc J 1976;115:8336.

18. Hickner J, Thompson PJ, Wilkinson T, Epner P, Sheehan M, Pollock AM, et al. Primary care physicians' challenges in ordering clinical laboratory tests and interpreting results. J Am Board Fam Med 2014;27:268-74.

19. Lenters-Westra E, den Besten G, Slingerland RJ. An unexpected low hba1c: Measurement error or interpretation problem? Clin Chem 2019;65:1062-3.

20. Lenters-Westra E, Schindhelm RK, Bilo HJ, Groenier KH, Slingerland RJ. Differences in interpretation of haemoglobin a1c values among diabetes care professionals. Neth J Med 2014;72:462-6. 
21. Fraser CG. Biological variation: From principles to practice. Amer Assoc for Clinical Chemistry 2001.

22. Carobene A, Marino I, Coskun A, Serteser M, Unsal I, Guerra E, et al. The eubivas project: Within- and between-subject biological variation data for serum creatinine using enzymatic and alkaline picrate methods and implications for monitoring. Clin Chem 2017;63:1527-36.

23. Ceriotti F, Hinzmann R, Panteghini M. Reference intervals: The way forward. Ann Clin Biochem 2009;46:8-17.

24. Coskun A, Sandberg S, Unsal I, Cavusoglu C, Serteser M, Kilercik M, Aarsand AK. Personalized reference intervals in laboratory medicine: A new model based on within-subject biological variation. Clin Chem 2020.

25. Katzman BM, Wockenfus AM, Scott RJ, Bryant SC, Jaffe AS, Karon BS. Estimating short- and long-term reference change values and index of individuality for tests of platelet function. Clin Biochem 2019;74:54-9.

26. Iglesias $\mathrm{N}$, Petersen $\mathrm{PH}$, Ricos $\mathrm{C}$. Power function of the reference change value in relation to cut-off points, reference intervals and index of individuality. Clin Chem Lab Med 2005;43:4418.

27. Fehr T, Milz P. The individuality index: A measure to quantify the degree of inter-individual, spatial variability in intra-cerebral brain electric and metabolic activity. Cogn Neurodyn 2019;13:429-36.

28. Fraser CG. Generation and application of analytical goals in laboratory medicine. Ann Ist Super Sanita 1991;27:369-75.

29. Fraser CG. Inherent biological variation and reference values. Clin Chem Lab Med 2004;42:758-64.

30. Fraser CG. Reference change values. Clin Chem Lab Med 2011;50:807-12.

31. Kawano R, Ichihara K, Wada T. Derivation of level-specific reference change values (rcv) from a health screening database and optimization of their thresholds based on clinical utility. Clin Chem Lab Med 2016;54:1517-29.

32. Bruins S, Fokkema MR, Romer JW, Dejongste MJ, van der Dijs FP, van den Ouweland JM, Muskiet FA. High intraindividual variation of b-type natriuretic peptide (bnp) and aminoterminal probnp in patients with stable chronic heart failure. Clin Chem 2004;50:2052-8.

33. Istaces N, Gulbis B. Study of fibrotest and hyaluronic acid biological variation in healthy volunteers and comparison of serum hyaluronic acid biological variation between chronic liver diseases of different etiology and fibrotic stage using confidence intervals. Clin Biochem 2015;48:652-7.

34. Quan H, Zhang B, Lan Y, Luo X, Chen X. Bayesian hypothesis testing with frequentist characteristics in clinical trials. Contemp Clin Trials 2019;87:105858.

35. Roraas T, Sandberg S, Aarsand AK, Stove B. A bayesian approach to biological variation analysis. Clin Chem 2019;65:995-1005.

36. Spiegelhalter DJ, Myles JP, Jones DR, Abrams KR. Bayesian methods in health technology assessment: A review. Health Technol Assess 2000;4:1-130.

37. Aarsand AK, Roraas T, Fernandez-Calle P, Ricos C, Diaz-Garzon J, Jonker N, et al. The biological variation data critical appraisal checklist: A standard for evaluating studies on biological variation. Clin Chem 2018;64:501-14. 


\section{Addendum}




\section{Summary Samenvatting Impact paragraph List of abbreviations List of publications Curriculum Vitae Dankwoord}




\section{Summary}

Two major purposes for using laboratory results are: 1) diagnosing or ruling out a disease and 2) monitoring changes in the clinical condition of a patient. When using laboratory results for the latter purpose, physicians often have to determine whether an observed change between serial measurements is a 'real change' or a 'random fluctuation'. Currently, physicians rely on their intuition and clinical experience, sometimes combined with using population based reference values. However, intuition and clinical experience are not the same across all physicians, and population based reference values based on between-subject biological variation are generally unsuitable for monitoring serial laboratory results within an individual.

To monitor changes in the clinical condition of a patient in a consistent and fact based way, the physician needs to apply principles of laboratory medicine in daily clinical practice. This thesis is about this intersection. It uses data on biomarkers to calculate within-subject biological variation estimates to facilitate evidence based approach for the monitoring of laboratory results over time. Further, it brings theoretical laboratory medicine findings to a user-friendly application that can help physicians to discriminate between 'real changes' and 'random fluctuations' when interpreting serial laboratory results.

This thesis starts with a general introduction and addresses four major topics. The general introduction (chapter 1) describes the 'clinical challenge' that physicians face when it comes to interpreting laboratory results in further detail, and touches upon the (ir)relevance of population-based reference values when it comes to medical decision making.

The first major topic is the development of the medical smartphone app Labtracker. This app calculates the probability of change between two serial measurements and provides an individualized and objective assessment of the likelihood of a true change. With this, Labtracker interprets the magnitude of change between serial laboratory results, while taking into account the biological variation of the observed parameter, the time interval between the measurements and the analytical variation. In chapter 2 , the background and underlying calculation algorithm of the medical smartphone app Labtracker is described. 
The second major topic concerns the provision of reliable data on within-day biological variation in healthy subjects that can extend the current datasets. Availability of data on within-subject biological variation is incomplete, which limits evidence-based interpretations of serial laboratory results in clinical practice. Chapters 3-7 provide a reference data set that extends the current knowledge of within-subject biological variation, to be used for calculations of Reference Change Values (RVC), with emphasis on the variation within 24 hours.

The third major topic concerns the comparison between within-subject biological variation in healthy and non-healthy subjects. A largely unverified assumption for the validity of using the RCV in clinical practice is that within-subject biological variation does not change substantially in various chronic diseases, compared to healthy subjects. It is assumed that the variability around a person's homeostatic set-point is relatively constant in health and chronic diseases. In chapters 4-7, we tested this assumption by comparing the within-subject biological variation from patients with Chronic Kidney Disease (CKD) and aortic valve stenosis with healthy subjects and did not identify biological variation shifts of a magnitude that would invalidate the use of RCV's, calculated with within-subject biological variation data from healthy subjects, in these patients.

Fourth, the external validation of the medical smartphone app Labtracker (chapter 8). We evaluated concordance rates of Labtracker calculations with medical students, medical residents and experienced physicians and showed progressively better concordance rates with Labtracker with increasing clinical experience. With this Labtracker may benefit clinical decisions related to monitoring of a patient.

Lastly, chapter 9 contains a general discussion of the work presented in this thesis and provides directions for further research. 


\section{Samenvatting}

Twee belangrijke doelen voor het gebruik van laboratoriumresultaten zijn: 1) het diagnosticeren of uitsluiten van een ziekte en 2) het volgen van veranderingen in de klinische toestand van een patiënt. Bij het gebruik van laboratoriumresultaten voor dit laatste doel moeten artsen vaak bepalen of een waargenomen verandering tussen opeenvolgende metingen een 'echte verandering' of een 'willekeurige fluctuatie' is. Momenteel vertrouwen artsen vaak op hun intuïtie en klinische ervaring, soms gecombineerd met het gebruik van op de bevolking gebaseerde referentiewaarden. Intuïtie en klinische ervaring zijn echter niet bij alle artsen hetzelfde, en op de populatie gebaseerde referentiewaarden op basis van biologische variatie tussen proefpersonen zijn over het algemeen niet geschikt voor het monitoren van seriële laboratoriumresultaten binnen een individu.

Om veranderingen in de klinische toestand van een patiënt op een consistente en op feiten gebaseerde manier te volgen, moet de arts de principes van laboratoriumgeneeskunde toepassen in de dagelijkse klinische praktijk. Dit proefschrift gaat over dit snijpunt. Het gebruikt gegevens over laboratoriumparameters om schattingen van biologische variaties binnen een proefpersoon te berekenen om een evidence-based benadering voor het monitoren van laboratoriumresultaten in de tijd te vergemakkelijken. Verder brengt het theoretische laboratoriumgeneeskundige bevindingen naar een gebruiksvriendelijke toepassing die artsen kan helpen onderscheid te maken tussen 'echte veranderingen' en 'willekeurige fluctuaties' bij het interpreteren van seriële laboratoriumresultaten.

Dit proefschrift begint met een algemene inleiding en behandelt vier hoofdonderwerpen. De algemene inleiding beschrijft de 'klinische uitdaging' waarmee artsen worden geconfronteerd als het gaat om het interpreteren van laboratoriumresultaten in meer detail, en gaat in op de (ir)relevantie van op de bevolking gebaseerde referentiewaarden als het gaat om medische besluitvorming.

Het eerste onderwerp is de ontwikkeling van de medische smartphone-app Labtracker. Deze app berekent de kans op verandering tussen twee opeenvolgende metingen en produceert een individuele en objectieve beoordeling van de kans op een echte verandering. Hiermee interpreteert Labtracker de omvang van de verandering tussen opeenvolgende laboratoriumresultaten, rekening houdend met de biologische variatie van de waargenomen parameter, het tijdsinterval 
tussen de metingen en de analytische variatie. In hoofdstuk 2 wordt de achtergrond en het onderliggende rekenalgoritme van de medische smartphone-app Labtracker beschreven.

Het tweede onderwerp in dit proefschrift betreft het leveren van betrouwbare gegevens over biologische variatie binnen de dag bij gezonde proefpersonen die de huidige datasets kunnen uitbreiden. De beschikbaarheid van gegevens over biologische variatie binnen een proefpersoon is momenteel onvolledig, wat op bewijs gebaseerde interpretaties van opeenvolgende laboratoriumresultaten in de klinische praktijk beperkt. Hoofdstukken 3-7 bieden een set aan gegevens die de huidige kennis van binnen-persoons biologische variatie uitbreidt, om te worden gebruikt voor berekeningen van het 'kritisch verschil', ook wel de reference change value (RVC) genoemd, met de nadruk op de variatie binnen 24 uur.

Het derde onderwerp in dit proefschrift betreft de vergelijking tussen biologische variatie binnen individuen bij gezonde en niet-gezonde individuen. Een grotendeels niet-geverifieerde aanname voor de validiteit van het gebruik van het kritisch verschil in de klinische praktijk is dat de biologische variatie binnen de patiënt niet substantieel verandert bij verschillende chronische ziekten, vergeleken met gezonde proefpersonen. Aangenomen wordt dat de variabiliteit rond het homeostatische setpoint van een persoon relatief constant is bij gezondheid en chronische ziekten. In de hoofdstukken 4-7 hebben we deze aanname getest door de biologische variatie binnen patiënten van patiënten met chronische nierziekte (CKD) en aortaklepstenose met gezonde proefpersonen te vergelijken en identificeerden we geen biologische variatie schommelingen van een omvang die het gebruik van het kritisch verschil, berekend met binnen-persoons biologische variatiegegevens van gezonde proefpersonen, bij deze patiënten.

Het vierde onderwerp in dit proefschrift betreft de externe validatie van de medische smartphone-app Labtracker (hoofdstuk 8). We evalueerden concordantie percentages van Labtracker-berekeningen met coassistenten, arts-assistenten en ervaren internisten en toonden progressief betere concordantie percentages met Labtracker met toename van klinische ervaring. Hiermee kan Labtracker klinische beslissingen met betrekking tot de monitoring van een patiënt ten goede komen.

Tenslotte bevat hoofdstuk 9 een algemene bespreking van het werk dat in dit proefschrift wordt gepresenteerd en het geeft suggesties voor verder onderzoek. 


\section{Impact paragraph}

In this last chapter the valorization of this thesis will be further explored. Valorization is the social utilization of scientific knowledge. It is about the why behind the research and is intended to make knowledge usable other than for scientific purposes only.

The valorization of this thesis consists of two parts. The first part of the valorization in this thesis is the development of the medical smartphone app Labtracker. The development of the smartphone app Labtracker boosts the valorization of the results of this thesis. This app helps its users to compare two laboratory results with each other in a fact based and scientifically sound manner, and facilitates the assessment of whether the change between these two results is a clinically relevant change or not. Specifically, Labtracker calculates the probability that an increase or decrease over time in a specific blood parameter is real, given the time between measurements. It reduces the need for physicians to rely on their clinical experience and intuition when they have to interpret whether a change between serial laboratory measurements is a "real" change or a natural fluctuation.

Another important part of the valorization of this thesis is the enrichment of the database on within-subject biological variation along two dimensions. First, researching additional/new parameters has increased the breadth of the database; second, the depth of the database has been increased for specific parameters, by looking at the difference between healthy and chronically ill individuals, and by looking at the difference in biological variation within a day.

Both the development of the app and the enrichment of the biological variation database can have an impact on three important themes: health care, education, and prevention.

\section{Healthcare}

By using Labtracker based calculations to distinguish between a real change and a natural fluctuation, concerning a change between serial laboratory measurements, a physician can make a more scientifically based choice in the field of laboratory diagnosis and with this, he can make better decisions.

Besides, healthcare may improve by avoiding unnessacary treatments and/ or diagnostics when using Labtracker, such as prescribing a cholesterol inhibitor in a person with a cholesterol increase (that may be due to biological fluctuation instead of a real cholesterol increase). 
Concerning the enrichment of the biological variation database, healthcare may improve by facilitating physicians with an extensive dataset of biological variation of laboratory parameters, with which they can easily detect a ' real change' in laboratory parameters that may be clinically relevant.

Possibly, in the future, the way of measuring laboratory parameters will change. Techniques such as body sensors or infrared methods are rapidly developing. When this data generation will be expanded, the biological variation database will be essential to deal with all these laboratory data.

\section{Prevention}

Through the broadening of the biological variation database and the development of Labtracker, physicians can make a better estimate of the variability of laboratory measurements over time and hereby, they can better predict and possibly prevent the development of a disease. With this, the results of this thesis open doors to ways of preventive healthcare. Also patient can use the app Labtracker for themselves, to monitor their laboratory values and compare them with each other. By detecting an abnormality in an early stage, prevention and early-stage treatments will become more important. And eventually, this may lead to less expensive treatments, and even more important, a more value based healthcare for the patient.

\section{Education}

Young or inexperienced doctors are quickly brought to a higher level of interpreting laboratory diagnostics and developing a clinical 'sense' or intuition whether a change between serial laboratory measurements is a real change.

This speeds up the growth and development of physicians. By using Labtracker (inexperienced) clinicians become more aware of the variation within laboratory results. This may lead to less incorrect interpretations of a change in serial laboratory results and to a decreasing amount of unneccesary treatments.

For example, this thesis shows the variablity of hemoglobin during the day. By having knowledge about the biological variation of such a parameter or using Labtracker (in which this kwowledge is incorporated), physicians can determine more easily whether or not it is necessary to give an iron supplement or a blood transfusion. The app Labtracker can serve as an educational tool to verify the clinical intuition and it can serve as a reference to check the (diurnal) biological variation of the parameter. 


\section{List of abbreviations}

\begin{tabular}{|c|c|}
\hline $\mathrm{RCV}$ & reference change value \\
\hline $\mathrm{CV}_{\mathrm{A}}$ & analytical coefficient of variation \\
\hline $\mathrm{CV}_{\mathrm{I}}$ & intra-individual coefficient of variation \\
\hline hsTnI & high sensitive troponin I \\
\hline hsTnT & high sensitive troponin T \\
\hline GP & general practicioner \\
\hline FT4 & free thyroxine 4 \\
\hline TSH & thyroid stimulating hormone \\
\hline CI & confidence interval \\
\hline II & index of individuality \\
\hline $\mathrm{CV}_{\mathrm{G}}$ & group (inter-individual) coefficient of variation \\
\hline MCV & mean corpuscular volume \\
\hline $\mathrm{MCH}$ & mean corpuscular hemoglobin \\
\hline $\mathrm{MCHC}$ & mean corpuscular hemoglobin concentration \\
\hline MPV & mean platelet volume \\
\hline IPF & immature platelet fraction \\
\hline PCT & plateletcrit \\
\hline PDW & platelet distribution width \\
\hline BMI & body mass index \\
\hline $\mathrm{HbA1c}$ & glycated hemoglobin A1c \\
\hline HDL & high-density lipoprotein \\
\hline LDL & low-density lipoproteine \\
\hline eGFR & estimated glomerular filtration rate \\
\hline EDTA & ethylenediaminetraacetic acid \\
\hline ANOVA & analysis of variance \\
\hline SEM & standard error of the mean \\
\hline CV & coefficient of variation \\
\hline CKD & chronic kidney disease \\
\hline STFR & soluble transferrin receptor \\
\hline LDH & lactate dehydrogenase \\
\hline CK & creatine kinase \\
\hline ASAT & asparate-aminotransferase \\
\hline ALAT & alanine-aminotransferase \\
\hline MDRD & modification of diet in renal disease \\
\hline CKD-EPI & chronic kidney disease epidemiology \\
\hline
\end{tabular}




$\begin{array}{ll}\text { ECG } & \text { electrocardiogram } \\ \text { BNP } & \text { brain natriuretic peptide } \\ \text { NT-pro BNP } & \text { N-terminal pro natriuretic peptide } \\ \text { ST2 } & \text { suppression of tumourigenicity } 2 \\ \text { AVS } & \text { aortic valve stenosis } \\ \text { LVEF } & \text { left ventricular ejection fraction } \\ \text { SD } & \text { standard deviation } \\ \text { IQR } & \text { inter quartile range } \\ \text { RIT } & \text { item test correlation } \\ \text { aPTT } & \text { activated tromboplastin time }\end{array}$




\section{List of publications}

Hilderink JM, van der Linden N, Kimenai DM, Litjens EJ, Klinkenberg LJJ, Aref, BM, Aziz F, Kooman JP, Rennenberg, RJMW Bekers O, Koopmans, RP, Meex SJR. Twenty-four hour biological variation of creatinine, cystatin C and estimated Glomerular Filtration Rate in subjects with or without Chronic Kidney Disease. Clin Chem. 2017 Oct;63(10):1655-1656. doi: 10.1373

Hilderink JM, Rennernberg RJMW, Vanmolkot FHM, Bekers, O Koopmans RP, Meex $\mathrm{SJR}$. Labtracker+, a medical smartphone app for the interpretation of consecutive laboratory results: an external validation study. BMJ Open. 2017 Sep 1;7(9):e015854. doi: 10.1136

van der Linden N, Hilderink JM, Cornelis T, Kimenai DM, Klinkenberg LJJ, van Doorn WP, Litjens EJR, van Suijlen JDE, van Loon LJC, Bekers O, Kooman JP, Meex SJR. Twenty-Four-Hour Biological Variation Profiles of Cardiac Troponin I in Individuals with or without Chronic Kidney Disease. Clin Chem. 2017 Oct;63(10):1655-1656. doi: 10.1373

Hilderink JM, Klinkenberg LJJ, Aakre KM, de Wit NCJ, Henskens YMC, van der Linden N, Bekers O, Rennenberg RJMW, Koopmans RP, Meex SJR. Within-day biological variation and hour-to-hour reference change values for hematological parameters. Clin Chem Lab Med. 2017 Jun 27;55(7):1013-1024. doi: 10.1515

Hilderink JM, Koopmans RP, Rennenberg RJ, van Dieijen-Visser MP, Meex SJ. Real change or natural fluctuation? Ned Tijdschr Geneeskd. 2016;160(0):D132.

Hilderink JM, Koopmans RP, Rennenberg RJWM, van Dieijen-Visser MP, Meex SJR. Calculating the probability of change between two consecutive laboratory test results. [Reply to: Explaining laboratory test results to patients: what the clinician needs to know by Marice John O'Kane and Berenice Lopez]. BMJ. 2015;351:h5552.

van der Linden N, Cornelis T, Klinkenberg LJ, Kimenai DM, Hilderink JM, Litjens EJ, Kooman JP, Bekers O, van Dieijen-Visser MP, Meex SJ. Strong diurnal rhythm of troponin T, but not troponin I, in a patient with renal dysfunction. Int J Cardiol. 2016 Oct 15;221:287-8. doi: 10.1016 
Hilderink JM, Klinkenberg LJJ, van der Linden N, Kimenai DM, Rennenberg RJMW, Bekers O, Koopmans RP, Meex SJR. Within-day biological variation of 26 biochemical parameters in subjects with and without chronic kidney disease. Submitted.

Peeters FECM, Kietselaer BLJH, Hilderink JM, van der Linden N, Niens M, Crijns HJGM, Meex SJR. Biological variation of cardiac markers in patients with aortic valve stenosis. Open Heart. 2019 May 8;6(1):e001040. doi: 10.1136

van der Linden N, Cornelis T, Kimenai DM, Klinkenberg LJJ, Hilderink JM, Lück S, Litjens EJR, Peeters FECM, Streng AS, Breidthardt T, van Loon LJC, Bekers O, Kooman JP, Westermark PO, Mueller C, Meex SJR. Origin of Cardiac Troponin T Elevations in Chronic Kidney Disease. Circulation. 2017 Sep 12;136(11):1073-1075. doi: 10.1161 


\section{Curriculum Vitae}

Judith Hilderink was born on October 4th, 1990 in Hengelo (GId), the Netherlands. She graduated from secondary school (VWO) at Ludgercollege Doetinchem. In 2009, she started studying medicine at Maastricht University.

In the fourth year of her study, Judith started working as a student assistant at the department of Clinical Chemistry, in collaboration with the department of Internal Medicine. During two years, she studied the biological variation of laboratory parameters, together with Prof. dr. Richard Koopmans, Prof. dr. Roger Rennenberg and dr. Steven Meex, which eventually led to

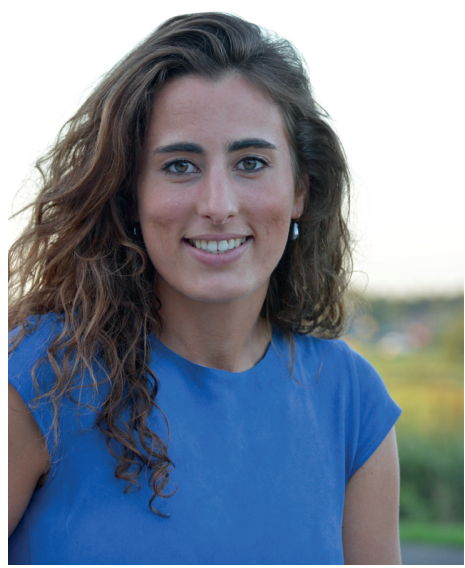
the development of the medical smartphone app Labtracker. After obtaining her medical degree in 2015, Judith continued as a PhD student in the departments Clinical Chemistry and Internal Medicine, supervised by Prof. dr. Otto Bekers, Prof. dr. Richard P. Koopmans, and dr. Steven J. R. Meex. The results of her PhD, described in this thesis, are published in various peer-reviewed journals, and presented at both national and international conferences.

In March 2018, Judith started working as a physician at the department of internal medicine at the IJsselland Ziekenhuis in Capelle aan den IJssel. She started her residency in Internal Medicine in 2019. From January $1^{\text {st. }}$ 2020, she continued her residency in the Erasmus Medical Center, Rotterdam. 


\section{Dankwoord}

Dit proefschrift was nooit tot stand gekomen zonder de hulp, inzet en steun van vele collega's, vrienden en familie gedurende de afgelopen jaren. De volgende personen wil ik graag in het bijzonder bedanken.

Allereerst mijn twee promotores, prof. dr. Otto Bekers en prof. dr. Richard Koopmans. Beste Otto, dank voor je steun en vertrouwen tijdens mijn promotietraject. Met je dropjes wist je het altijd gezellig te maken tijdens de research meetings. Ik waardeer je pragmatische manier van aanpak; als jij iets moest regelen voor mijn proefschrift was het vaak dezelfde dag nog rond.

Richard, jouw enthousiasme en gedrevenheid zijn ongeëvenaard. Je bent altijd erg begaan geweest met mij en met mijn onderzoek. Als ik even niet goed wist hoe ik verder moest hoefde ik maar even bij je langs te gaan, plaats te nemen op je paarse bank en ik liep even later met een grote glimlach én vol nieuwe ideeën de deur uit.

Mijn co-promotor, dr. Meex, beste Steven, bij jou is het allemaal begonnen tijdens mijn geneeskunde studie met een les over zuur-base evenwichten. Vervolgens wees je me de weg in de wondere wereld van wetenschappelijk onderzoek. Dat die eerste stappen uiteindelijk zouden leiden tot een heus proefschrift had ik destijds nooit durven dromen. Ik ben ervan onder de indruk hoe je je drukke leven als klinisch chemicus hebt weten te combineren met het begeleiden van (destijds 5!) promovendi en een gezinsleven. Je hebt me de afgelopen jaren vrijwel onbegrensde vrijheid gegeven en ik heb ontzettend veel van je geleerd. Heel veel dank Steven!

Mijn paranimfen, Dorien en Evelyne. Dorien, lieve roomie, vanaf de eerste dag van mijn wetenschapsstage was jij mijn buddy (samen in de bezemkast, onder het genot van het foute uur). Ik heb zo veel aan je gehad en van je geleerd. Jouw eerlijke, respectvolle en integere houding, zowel als collega, als wetenschapper en als vriendin, vind ik enorm bewonderenswaardig. Het was fijn om altijd iemand te hebben om op terug te vallen en ben dan ook heel blij dat je me bij wilt staan als paranimf. We hebben in die 2,5 jaar dat we roomies waren heel wat successen gevierd (met foute drank). Ik had me geen betere roomie kunnen wensen. Wat ben ik blij dat ik jou heb leren kennen.

Lieve Evelyne, dankjewel dat jij mijn paranimf wilt zijn. Al sinds de bachelor van onze geneeskunde studie kan ik altijd op je terug vallen. Met heel veel plezier 
kijk ik terug op al onze sportavonden en aansluitende etentjes (bij voorkeur een salade valdieu na het zwemmen ;-)). Na onze studies hebben we dit voortgezet en onze ervaringen als promovendi gedeeld. Gelukkig zien we elkaar nog steeds regelmatig. Ik bewonder je doorzettingsvermogen en positieve instelling. Ik hecht veel waarde aan onze vriendschap en kijk uit naar vele mooie jaren die nog komen gaan.

Prof. dr. Rennenberg, beste Roger, vanaf de eerste dag was je betrokken bij de brainstormsessies, en later de ontwikkeling van Labtracker. Jouw nuchtere kijk werkte voor mij vaak verhelderend.

Prof. dr. van Dieijen, beste Marja, ook al ben je officieel geen onderdeel van mijn promotieteam, maar ik heb (met name in het begin van mijn promotie onderzoek) erg veel aan je gehad. Jij hebt het unieke talent om na een periode van afwezigheid in een paar minuten en door de juiste vragen te stellen, volledig op de hoogte te zijn van de stand van zaken rondom mijn promotieonderzoek.

Leden van de beoordelingscommissie, Prof. dr. Walther van Mook, Prof. dr. Fred Sweep, Prof dr. Jan-Joost Rethans, Prof. dr. Eric Sijbrands en Prof. dr. Bram Kroon. Ik waardeer het zeer dat $u$ allen plaats wilt nemen in de beoordelingscommisie. Dank voor de nauwkeurige beoordeling van mijn proefschrift.

Tevens wil ik de overige leden van de commissie bedanken voor de bereidheid om als opponent deel te nemen. Ik kijk er naar uit om met u van gedachten te wisselen tijdens de verdediging.

Dr. Bart de Wit, Dr. ir. Yvonne Henskens, Dr. Judith Bons, Dr. Alma Mingels, Dr. Jan Damoiseaux, Dr. Irene Korver-Keularts, Dr. Douwe de Boer, Dr. Will Wodzig, Dr. Joyce van Beers, Dr. ir. Sander Streng bedankt voor jullie interesse in mijn onderzoek en de kritische vragen tijdens de refereer sessies. Ik heb, mede dankzij jullie, een enorm leuke tijd gehad op het CDL.

Dat je onderzoek nooit alleen doet blijkt wel uit de vele co-auteurs die allen op hun eigen manier een bijdrage hebben geleverd aan de artikelen. Veel dank voor jullie hulp, commentaar en verbeteringen van onze research. Een aantal zou ik graag in het bijzonder willen bedanken. Dr. Kristin Aakre; early on in my PhD, we contacted you to brainstorm about the possibility of hour-to-hour calculations. You 
have always been very helpful and you have made an essential contribution to the piece on hematological parameters.

Dr. Floris Vanmolkot, zonder aarzelen heb je de planning van de terugkomdagen van het co-schap interne geneeskunde aangepast zodat ik mijn 'labtrackerquiz' kon afnemen bij de coassistenten. Prof. Dr. Jeroen Kooman; ik geloof oprecht dat jouw kritische heldere commentaren op het manuscript over de nefrologische parameters het stuk naar een hoger niveau getild hebben

Vincent, ik, als kneusje op het lab keek in het begin mijn ogen uit ("Oh, is dat plasma? Ik dacht urine") Als manusje van alles wist jij alle problemen en storingen (en die hebben we gehad!) altijd weer op te lossen. Je was onmisbaar tijdens de dagen op het lab, heel veel dank voor je hulp en gezelligheid

Petal, jij bracht altijd leven in de brouwerij en wist op jouw eigenzinnige manier kleur te geven aan de dagen op het lab. Dank voor je gezelligheid op het lab en op borrels.

Een zeer belangrijk aandeel in dit promotietraject is weggelegd voor mijn collegaonderzoekers. Allereerst mijn 'voorgangers' Lieke, Sander, Noreen en Linda. Jullie waren allen bezig met de afrondingen van jullie promotie onderzoeken toen ik begon als promovendus. Bedankt voor jullie goede voorbeelden en collegiale hulp. Heel veel succes in jullie verdere carrières.

Frederique, wat was je altijd een fijn gezelschap tijdens meetings, lunches en borrels. Jouw nauwkeurigheid en oog voor detail zijn voor mij een voorbeeld. Succes met jouw opleiding tot cardioloog!

Stephanie, bedankt voor de gezellige tijd. Heel veel succes met de laatste loodjes van jouw promotie onderzoek.

William (Willy), het was nooit saai met jou als collega. Altijd had je weer wat grappigs beleefd in het weekend en langzaam maar zeker werd je steeds volwassener. Ik bewonder je pragmatische aanpak en je ambitie. Ik kijk er naar uit om binnenkort weer naar Maastricht te rijden voor jouw verdediging! 
Wim, zelden heb ik zo een gedreven persoon als jij ontmoet. Dank voor de gezelligheid, de vele koffies en de fijne sfeer in onze werkkamer. Ik vond het inspirerend om te zien hoe jij tijdens jouw promotie onderzoek een heel helder doel voor ogen had en daar recht op af ging. Heel veel succes met het vervolg van je opleiding tot klinisch chemicus, en natuurlijk ook met je leven als kersverse papa.

Charlotte, Geneviève en Dorien, Lumière clubje, wat was het iedere keer weer gezellig om af te spreken en de ervaringen, belevenissen en frustraties van onze onderzoeken te kunnen delen. We hebben samen de nodige films gezien, colleges bijgewoond en koffies gedronken. Als laatste in ons groepje mag ik nu ook mijn proefschrift bij de verzameling voegen.

Dr. Emile De Bruijne, bedankt dat ik mijn opleiding tot internist in het IJsselland Ziekenhuis mocht starten. Ik heb onze samenwerking altijd als zeer prettig ervaren.

Dr. Adrienne Zandbergen en Prof. Dr. Stephanie Klein-Nagelvoort, bedankt dat jullie me zo fijn hebben 'opgevangen' in het EMC en dat jullie me direct lieten thuis voelen in zo'n grote organisatie.

Rianne en Nienke, ook al zijn we alle drie onze eigen weg gegaan na onze gezamenlijke turnjaren, we zijn elkaar nooit uit het oog verloren. Bedankt voor jullie fijne vriendschap.

Anne, wat ben ik blij dat we na onze jaren als Rowing Stones bij Saurus nog steeds contact hebben. Fijn dat ik altijd even kon uitstappen in Eindhoven voor een gezellig etentje 'op de route' als ik weer eens aan het pendelen was tussen Rotterdam en Maastricht. Ik prijs me gelukkig met jou als vriendin.

Veerle, je bent als familie voor me, maar vooral een hele waardevolle vriendin. Alles delen en bespreken we samen tijdens onze wekelijkse hardloop (en inmiddels wandel ;-)) rondjes. Dankjewel voor je luisterende oor en je goede adviezen. Jouw nuchtere kijk op het leven in combinatie met jouw heerlijke humor hebben mij vaak nieuwe inzichten gegeven.

Sophie, Eva, Saskia en Jessica, wat ben ik blij dat we na ons gezamenlijke jaar als ANIOS in het IJsselland nog steeds contact hebben, ook al zijn we allemaal een 
andere kant op gegaan. Dank voor alle gezellige etentjes, escape rooms, (zoom-) bijklets sessies en nog veel meer.

Naomi, ook jij bent een hele fijne vriendin geworden. Altijd recht door zee, goudeerlijk en vol plannen. Fijn dat we elkaar nu in het EMC weer kunnen opzoeken.

Maarten en Cilia, vanuit het IJsselland gingen we met z'n drieën naar het EMC. Wat fijn dat we dit samen hebben kunnen doen en dat we elkaar nog geregeld opzoeken met koffies.

Susan, Ellen, Josje en Frida, sinds de middelbare school al mijn lieve vriendinnen waarmee ik alles kan delen. Van gezellige pauzes in de Kandeleer tot allemaal ons eigen leven in een andere stad; ik ben heel blij dat onze vriendschap er na al die jaren nog steeds is. Snel weer eens met de vijf op avontuur!

Ton en Ellen, al heel wat jaren kom ik bij jullie over de vloer en het voelt als een warm bad als ik bij jullie ben. Jullie zijn vanaf het eerste moment geïnteresseerd geweest in mijn promotie onderzoek. Bedankt voor het meedenken over toekomstplannen en voor al jullie wijze adviezen.

Caspar en Nina, wat fijn om jullie ook familie te mogen noemen. Jullie zijn een fantastisch duo!

Oma, dankjewel voor je oprechte interesse en belangstelling in ons leven. Ik vind het wonderbaarlijk dat je van al je (15!) kleinkinderen altijd precies kunt vertellen hoe het met iedereen gaat.

'Zwagers' Alexander, Martijn en Stein, wat een gezellige toevoeging zijn jullie aan onze familie. Fijn dat jullie mijn zussen zo gelukkig maken.

Anna, Olaf en Benjamin, de nieuwste generatie, jullie maken onze familie nog mooier, groter èn grappiger. Ontdek het leven en geniet!

Michelle, Lenny en Iris, liefste zussen, jullie kennen mij als geen ander. Alle vier zijn we zo verschillend, en toch delen we veel interesses. Ik bewonder jullie allemaal op jullie eigen manier en ik ben heel blij dat ik jullie heb. 
Papa en mama, bedankt voor jullie steun en vertrouwen. Jullie hebben mij altijd vrijgelaten in keuzes die ik wilde maken en me hierin laten inzien dat ik op mezelf kan vertrouwen. Jullie staan altijd voor mij klaar met (praktische) hulp en ondersteuning. Bij elke verhuizing kon ik weer op jullie rekenen. Bedankt voor al jullie zorgen en voor het bieden van de mogelijkheid om in Keijenborg altijd weer helemaal tot rust te komen.

En tot slot, lieve Rogier, de laatste woorden van dit dankwoord zijn vanzelfsprekend voor jou. Bedankt voor je onvoorwaardelijke liefde, begrip en vertrouwen. Al meer dan tien jaar ben je mijn steun en toeverlaat. Je gaf mij de tijd en ruimte die ik nodig had om naast een voltijd baan ook een promotie af te ronden. Je was altijd bereid om iets voor me na te lezen, samen te brainstormen, en vooral om me even te laten afschakelen van alles wat met werk- en PhD te maken had. Wat hebben we het fijn samen en wat ben ik blij dat we ons avontuur binnenkort met z'n drieën mogen voortzetten. Ik had het niet beter kunnen treffen! 